\title{
A FIRST PRINCIPLES BASED METHODOLOGY FOR DESIGN OF AXIAL COMPRESSOR CONFIGURATIONS
}

\author{
A Dissertation \\ Presented to \\ The Academic Faculty
}

by

\author{
Vishwas Iyengar
}

\begin{abstract}
In Partial Fulfillment of the Requirements for the Degree

Doctor of Philosophy in the School of Aerospace Engineering
\end{abstract}

Georgia Institute of Technology August 2007 


\section{A FIRST PRINCIPLES BASED METHODOLOGY FOR DESIGN OF AXIAL COMPRESSOR CONFIGURATIONS}

Approved by:

Dr. Lakshmi N. Sankar, Advisor School of Aerospace Engineering Dr. Richard Gaeta Georgia Institute of Technology School of Aerospace Engineering Georgia Institute of Technology

Dr. J.V.R. Prasad

Dr. Alex Stein

School of Aerospace Engineering Energy and Propulsion Technologies Georgia Institute of Technology GE Global Research Center

Dr. Dimitri Mavris

School of Aerospace Engineering Georgia Institute of Technology 
Dedicated to my parents

Mr. Manmohan Ram Iyengar and

Mrs. Revathy Iyengar

For their encouragement and endless love 


\section{ACKNOWLEDGEMENTS}

Over the years many people have made an impact on my life, not only from an academic point of view, but their friendship and support has helped me in many ways to complete this work. It has been a very long enduring journey!

I would like to especially thank Dr. Lakshmi N. Sankar, my thesis advisor and mentor, for his guidance and support throughout my research stint. His patience, support and kindness with his vast knowledge in research played a crucial role in my development at Georgia Tech. I thoroughly enjoyed working under him and would like to thank him for making my graduate school experience a valuable one.

I would also like to thank Dr. J. V. R. Prasad, Dr. D. Mavris, Dr. R. Gaeta and Dr A. Stein for their services as members of my thesis committee. Their valuable comments and suggestions encouraged me to move forward confidently in my research work, for which I am very grateful.

I am very grateful to Dr. Rodrick V. Chima at NASA Glenn Research Center for his ever-extended assistance in my research. His immense knowledge in compressor aerodynamics was very much appreciated and I would like to thank him for sharing it with me.

I would like to express my gratitude to Mr. Russell Denney at Aerospace Systems Design Lab- Georgia Tech for all his support and valuable advice on several issues.

Working at Georgia Tech has been a thoroughly challenging, yet enjoyable, experience. I have been supported throughout this experience by many special people. I would like to thank the past and present members of the CFD lab- Dr. Liu Yi, Dr. Sarun 
Benjanirat, Dr. Chanin Tongchitpakdee, Dr. Roxanna Vasilescu, Mina Zaki, Nandita Yeshala, Nischint Rajmohan, Sujeet Phanse, Byung Young Min, Jeremy Bain, Karthik Mani, Joseph Gillman- for their warm friendship and support.

I must also thank Dr. J. I. Jagoda, the graduate coordinator, and the staff of the Aerospace Engineering Office: Loretta Carroll, Daurette Joseph, Carole Gaines, and Rebecca Trout for always being ready with school related assistance.

I would like to thank my friends at school- Susheel Kumar Sekhar, Satish Undapalli, Preetham, Sachin Jain- for their company at the endless hours of coffee and lunch breaks. I would also like to thank my close friends- Sumier Phalake, Chetan Bagga, Gayathri Gunasekaran, Yemuna Balajiprasad- for always being there for me, especially during some rough times. A special note of thanks for my housemates and friends- Arpit Bhoti and Toral Shah- thank you for putting up with everything!

I would like to thank my entire extended family in India, United Kingdom and United States. I must thank my maternal and paternal grandmothers for their continuous blessing and affection. I express my hearty appreciation to my younger brother and friend, Vybhav Iyengar. Vybhav's motivation, grit and hardwork have always been influential to me and for that I thank him.

Finally, I owe my greatest gratitude to my parents, Manmohan Ram Iyengar and Revathy Iyengar, to whom this work is dedicated. My father's determination and my mother's patience have been a source of inspiration in my life. Their constant encouragement and great unconditional love made me who I am and gave me the strength to get through this incredible journey. Thanks! 


\section{TABLE OF CONTENTS}

$\begin{array}{lll}\text { Page } & \text { Pan }\end{array}$

ACKNOWLEDGEMENTS $\quad$ iv

LIST OF TABLES $\quad$ ix

LIST OF FIGURES $\quad$ x

LIST OF SYMBOLS AND ABBREVIATIONS XV

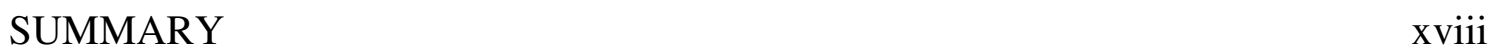

\section{CHAPTER}

1 INTRODUCTION 1

1.1 Understanding the Compressor Operation 2

1.2 Stability of Compression System 4

1.3 Compressor Design Overview 5

1.4 Objectives and Organization of the Present Work 7

2 DESIGN OF AXIAL FLOW COMPRESSORS 11

2.1 Computational Studies on Compressor Performance 11

2.2 Background of Compressor Design Methods 13

2.3 System Level Engine Design Overview 18

2.4 Literature on Optimization Techniques $\quad 18$

2.5 Overview of Compressor Aeroacoustics Techniques 21

2.6 Presently Used Design Methodology 25

3 MATHEMATICAL FORMULATION AND TOOLS 27

$\begin{array}{ll}3.1 \text { Governing Equations } & 28\end{array}$

3.2 Initial Conditions 33

3.3 Boundary Conditions 34 
3.3.1 Inflow Boundary Conditions

3.3.2 Outflow Boundary Conditions

3.3.3 Surface Boundary Conditions $\quad 35$

3.3.4 Rotor-Stator Interface Boundary Conditions 36

3.4 Time Marching Algorithm 39

3.5 Turbulence Model 40

3.6 Grid Generation Technique 41

3.7 Design Tools 42

3.7.1 Parametric Blade Design 43

3.7.2 Design of Experiments $\quad 44$

3.7.3 Response Surface Methodology $\quad 45$

3.8 Total Pressure Ratio and Adiabatic Efficiency Calculation 48

3.9 Aeroacoustic Analysis Technique 50

3.10 Structural Analysis $\quad 52$

3.11 Selection of Design Variables $\quad 52$

4 VALIDATION STUDIES $\quad 60$

4.1 NASA Transonic Axial Rotor $67 \quad 60$

4.2 Goldman Turbine Vane $\quad 62$

4.3 Space Shuttle Main Engine (SSME) Single Stage Turbine 63

4.4 Baseline Stage 35 Configuration 65

5 RESULTS I: PRELIMINARY APPLICATION OF METHODOLOGY 75

5.1 Design Variables 76

5.2 Comparison between Stage35 and Starting Configuration 76

$\begin{array}{ll}5.3 \text { Optimization Results } & 78\end{array}$

5.3.1 Performance map comparisons between Starting and Preliminary Configurations 
5.3.2 Flowfield comparisons between Starting and Preliminary

Configurations

6 RESULTS II: COMPREHENSIVE APPLICATION OF METHODOLOGY 96

6.1 Selection of Design Variables 97

6.2 Optimization Results 998

6.2.1 Assessment of blade loading $\quad 100$

6.2.2 Performance map comparisons between Starting and Optimized Configurations

6.2.3 Rotor-Stator aeroacoustic interaction comparisons between Starting and Optimized Configurations

6.2.4 Flowfield comparisons between Starting and Optimized Configurations

7 CONCLUSIONS AND RECOMMENDATIONS

7.1 Conclusions 


\section{LIST OF TABLES}

Page

Table 3.1: Design of experiment cases for the 18 design variables investigated 56

Table 5.1: Optimized values of rotor design variables used in preliminary application of methodology

Table 5.2: Optimized values of stator design variables used in preliminary application of methodology

Table 5.3: Differences observed between predicted and computed values for the objective functions in simple application of methodology

Table 6.1: Summary of the 18 design variables considered for the screening study

Table 6.2: Final design variables selected from screening studies

Table 6.3: Design of Experiment generated from the ten design variables selected

Table 6.4: Optimized values of rotor design variables used in comprehensive application of methodology

Table 6.5: Optimized values of stator design variables used in comprehensive application of methodology

Table 6.6: Differences observed between predicted and computed values for the three objective functions in comprehensive application of methodology 


\section{LIST OF FIGURES}

Page

Figure 1.1: Various components of a commonly used turbo-engine

Figure 1.2: Schematic diagram of rotor-stator configuration and changes in fluid properties through a stage

Figure 1.3: Typical performance map for an axial compressor with stability issues

Figure 1.4: Compressor geometry design with endwalls and blade rows

Figure 2.1: Box diagram showing the proposed methodology

Figure 2.2: Flowchart showing the sequence of events in the proposed design methodology

Figure 3.1: Control Volume and cell-vertex grid points

Figure 3.2: Cartesian to Cylindrical coordinates transformation

Figure 3.3: Schematic diagram showing the implementation of characteristic boundary condition at rotor-stator interface

Figure 3.4: Summary of parametric blade design based on design variables- $a_{M, N}, b_{M, N}$

Figure 3.5: Schematic diagram showing the interface location used for the aeroacoustic analysis

Figure 3.6: An example to illustrate the Pareto Curve

Figure 4.1: A nominal H-type computational grid for the Rotor 67 configuration

Figure 4.2: Characteristic performance map validating the CFD with the experimental results for Rotor 67 configuration

Figure 4.3: Characteristic efficiency map validating the CFD with the experimental results for Rotor 67 configuration

Figure 4.4: C-type computational grid for the Goldman Turbine Vane Configuration 68

Figure 4.5: Mach number contour comparison between CFD and published data at midspan location for Goldman Turbine Vane 
Figure 4.6: Comparisons between the experimental and CFD blade surface pressure distribution for the Goldman Turbine Vane

Figure 4.7: Sectional view of the computational grid used for SSME Turbine stage analysis

Figure 4.8: Mach number contours comparison between published data and CFD at midspan location for the SSME Turbine stage

Figure 4.9: Comparisons between previously published and CFD reproduced normalized pressure on blade surface for the SSME Turbine stage.

Figure 4.10: Comparisons between experimental and computed Stanton number on stator blade surface for the SSME Turbine stage

Figure 4.11: Comparisons between experimental and computed Stanton number on rotor blade surface for the SSME Turbine stage

Figure 4.12: Stage 35 multi-block grid used in the simulations

Figure 4.13: Rotor tip clearance grid for Stage 35

Figure 4.14: Characteristic performance map comparing Starting Configuration with the original Stage 35 configuration and the experimental data

Figure 5.1: Design variables (camber angles) used for the simple application of methodology

Figure 5.2: Rotor blade profile comparisons between the Stage 35 and starting configurations at hub, mid-span and tip locations

Figure 5.3: Stator blade profile comparisons between the Stage 35 and starting configurations at hub, mid-span and tip locations

Figure 5.4: Rotor blade profile comparisons between the starting and preliminary configurations at hub, mid-span and tip locations

Figure 5.5: Stator blade profile comparisons between the starting and preliminary configurations at hub, mid-span and tip locations

Figure 5.6: Performance map comparing the starting configuration to the preliminary configuration

Figure 5.7: Adiabatic efficiency map comparing the starting configuration to the preliminary configuration

Figure 5.8: Total temperature ratio map comparing the starting configuration to the preliminary configuration 
Figure 5.9: Schematic of the blade-to-blade passage for a typical axial compressor configuration

Figure 5.10: Entropy (non-dimensional) contour comparison between the (a). Starting and (b). Preliminary configurations, for mid passage location at off-design condition $\left(\right.$ mdot $=0.87$ mdot_choke, $\left.T_{\text {ref }}=288.3 \mathrm{~K}, \rho_{\text {ref }}=1.233 \mathrm{~kg} / \mathrm{m}^{3}\right)$

Figure 5.11: Entropy (non-dimensional) contour comparison between the (a). Starting and (b). Preliminary configurations, for tip clearance section at off-design condition $\left(\right.$ mdot $=0.87$ mdot_choke, $\left.\mathrm{T}_{\text {ref }}=288.3 \mathrm{~K}, \rho_{\text {ref }}=1.233 \mathrm{~kg} / \mathrm{m}^{3}\right)$

Figure 5.12: Mach number contour comparison between the (a). Starting and (b). Preliminary configurations at $90 \%$ span from hub at design condition ( $\mathrm{mdot}=$ 0.97mdot_choke)

Figure 5.13: Mach number contour comparison between the (a). Starting and (b). Preliminary configurations at $90 \%$ span from hub at off-design condition (mdot $=0.87$ mdot_choke)

Figure 5.14: Mach number contour comparison between the (a). Starting and (b). Preliminary configurations at tip off-design condition (mdot $=$ 0.87mdot_choke) and two meridional planes above $70 \%$ span

Figure 6.1: 18 design variables (camber angles) used for the comprehensive application of methodology

Figure 6.2: Pareto curve obtained with total pressure ratio as the objective function 118

Figure 6.3: Pareto curve obtained with adiabatic efficiency as the objective function. 119

Figure 6.4: Pareto curve obtained with rotor wake influence coefficient (RWIC) as the objective function

Figure 6.5: Rotor blade profile comparisons between the starting and optimized configurations at (a) hub, (b) mid-span and (c) tip locations

Figure 6.6: Stator blade profile comparisons between the starting and optimized configurations at (a) hub, (b) mid-span and (c) tip locations

Figure 6.7: Response surface for objective functions a). total pressure ratio, b). adiabatic efficiency and c). rotor wake influence coefficient, as a function of design variables $A$ and $B$.

Figure 6.8: Rotor blade loading curve comparison at (a) Hub, (b) Mid Span and (c) Tip between the starting configuration and optimized configuration

Figure 6.9: Stator blade loading curve comparison at (a) Hub, (b) Mid Span and (c) Tip between the starting configuration and optimized configuration 
Figure 6.10: Performance map comparing the starting configuration to the optimized configuration

Figure 6.11: Adiabatic efficiency map comparing the starting configuration to the optimized configuration

Figure 6.12: Total temperature ratio map comparing the starting configuration to the optimized configuration

Figure 6.13: Performance maps at 70\% RPM for the starting configuration and the optimized configuration

Figure 6.14: Comparisons of the blade-to-blade pressure fluctuation influencing the rotorwake/stator interactions aft of the rotor blade at mid-span

Figure 6.15: Comparisons of the blade-to-blade pressure fluctuation influencing the rotorwake/stator interactions ahead of the rotor blade at mid-span

Figure 6.16: Comparisons of the hub-to-tip pressure fluctuations influencing the rotorwake/stator interactions at rotor-stator interface

Figure 6.17: Direction and entropy strength of the rotor leading edge tip vortex

Figure 6.18: Entropy (non-dimensional) contour comparison between the (a). Starting and (b). Optimized configurations, for mid passage location at off-design condition $\left(\right.$ mdot $=0.87 \mathrm{mdot} \_$choke, $\left.T_{\text {ref }}=288.3 \mathrm{~K}, \rho_{\text {ref }}=1.233 \mathrm{~kg} / \mathrm{m}^{3}\right)$

Figure 6.19: Entropy (non-dimensional) contour comparison between the (a). Starting and (b). Optimized configurations, for tip clearance section at off-design condition $\left(\right.$ mdot $=0.87 \mathrm{mdot} \_$choke, $\left.T_{\text {ref }}=288.3 \mathrm{~K}, \rho_{\text {ref }}=1.233 \mathrm{~kg} / \mathrm{m}^{3}\right)$

Figure 6.20: Mach number contour comparison between the (a). Starting and (b). Optimized configurations at 90\% span from hub at off-design condition (mdot $=0.87$ mdot_choke)

Figure 6.21: Mach number contour comparison between the (a). Starting and (b). Optimized configurations at tip off-design condition ( $\mathrm{mdot}=0.87 \mathrm{mdot} \_$choke) and two meridional planes above $70 \%$ span

Figure 6.22: Rotor blade deflections showing the "hot" shape and the "cold" shape for the optimized configuration

Figure 6.23: Rotor blade displacements on (a). Pressure surface and (b). Suction surface for the Optimized configuration

Figure 6.24: von Mises stress on rotor blade (a). Pressure surface and (b). Suction surface for the Optimized configuration 
Figure 6.25: von Mises stress on stator blade suction surface for the Optimized configuration

Figure 6.26: Performance map comparing the baseline Stage 35 configuration to the Stage 35-2 configuration

Figure 6.27: Adiabatic efficiency map comparing the baseline Stage 35 configuration to the Stage 35-2 configuration

Figure 6.28: Rotor blade loading curve comparison at mid span between the Stage 35 configuration and Stage 35-2 configuration.

Figure 6.29: Stator blade loading curve comparison at mid span between the Stage 35 configuration and Stage 35-2 configuration. 


\section{LIST OF SYMBOLS AND ABBREVIATIONS}

$a_{i}$

$a_{M, N}$

$A_{i}$

$b_{i i}$

$b_{M, N}$

$c$

$c_{0 \text { ref }}$

$C_{i}$

$C_{P}$

$C_{V}$

$d_{i}$

$D$

$D_{a}$

E

$F_{I}, G_{I}, H_{I}$

$F_{V}, G_{V}, H_{V}$

$J$

$K$

$p$

$P_{i}$
Regression coefficients

Blade mean camber line angle

Weighting assigned to response

Respective regression coefficients of subsequent terms

Blade thickness

Speed of sound

Reference total sonic velocity

Characteristic variables

Specific heat at constant pressure

Specific heat at constant volume

Transformed response

Desirability function

Artificial dissipation term

Energy

Cartesian components of the inviscid flux vectors

Cartesian components of the viscid flux vectors

Jacobian of transformation

Thermal conductivity

Pressure

Point pressure 


\begin{tabular}{|c|c|}
\hline $\operatorname{Pr}$ & Prandtl number \\
\hline$q$ & Conserved flow variables \\
\hline$q_{L}, q_{R}$ & Left and right fluxes crossing a cell face \\
\hline$Q$ & Total velocity \\
\hline$R$ & Gas constant \\
\hline$R^{-}$ & Riemann invariant \\
\hline$R_{i}$ & Response \\
\hline$R_{I}$ & Inviscid residual \\
\hline$R_{V}$ & Viscous residual \\
\hline $\operatorname{Re}$ & Reynolds number \\
\hline$R W I C$ & Rotor Wake Influence Coefficient \\
\hline$S$ & Blade section \\
\hline$t$ & Time \\
\hline$T$ & Temperature \\
\hline$u, v, w$ & Absolute components of velocity \\
\hline$u^{\prime}, v^{\prime}, w^{\prime}$ & Relative components of velocity \\
\hline$U^{\prime}, V^{\prime}, W^{\prime}$ & Contravariant velocity \\
\hline$x, y, z$ & Cartesian coordinates \\
\hline
\end{tabular}

\section{Greek Symbols}

$\alpha_{i}$

$\gamma$

$\kappa$
Runge-Kutta stage coefficient

Ratio of specific heats

Von-Karman constant 


\section{Subscripts}

0

AMP

eff

lam

$\max$

$M, N$

ref

T, turb

$x, y, z, t, \xi, \eta, \zeta, \tau, v, \theta$

$\infty$

\section{Overbars}

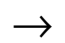

Stagnation quantity

Amplitude

Effective

Laminar

Maximum

Chord location, Span location

Reference

Turbulence quantity

Derivative with respect to

Freestream condition
Used to indicate vectors

Time or spatial average 


\section{SUMMARY}

Axial compressors are widely used in many aerodynamic applications. The design of an axial compressor configuration presents many challenges. Until recently, compressor design was done using 2-D viscous flow analyses that solve the flow field around cascades or in meridional planes or 3-D inviscid analyses. With the advent of modern computational methods it is now possible to analyze the 3-D viscous flow and accurately predict the performance of 3-D multistage compressors. It is necessary to retool the design methodologies to take advantage of the improved accuracy and physical fidelity of these advanced methods.

In this study, a first-principles based multi-objective technique for designing single stage compressors is described. The study accounts for stage aerodynamic characteristics, rotor-stator interactions and blade elastic deformations. A parametric representation of compressor blades that include leading and trailing edge camber line angles, thickness and camber distributions was used in this study A design of experiment approach is used to reduce the large combinations of design variables into a smaller subset. A response surface method is used to approximately map the output variables as a function of design variables. An optimized configuration is determined as the extremum of all extrema.

This method has been applied to a rotor-stator stage similar to NASA Stage 35. The study has two parts: a preliminary study where a limited number of design variables were used to give an understanding of the important design variables for subsequent use, and a comprehensive application of the methodology where a larger, more complete set 
of design variables are used. The extended methodology also attempts to minimize the acoustic fluctuations at the rotor-stator interface by considering a rotor-wake influence coefficient (RWIC). Results presented include performance map calculations at design and off-design speed along with a detailed visualization of the flow field at design and off-design conditions.

The present methodology provides a way to systematically screening through the plethora of design variables. By selecting the most influential design parameters and by optimizing the blade leading edge and trailing edge mean camber line angles, phenomenon's such as tip blockages, blade-to-blade shock structures and other loss mechanisms can be weakened or alleviated. It is found that these changes to the configuration can have a beneficial effect on total pressure ratio and stage adiabatic efficiency, thereby improving the performance of the axial compression system. Aeroacoustic benefits were found by minimizing the noise generating mechanisms associated with rotor wake-stator interactions. The new method presented is reliable, low time cost, and easily applicable to industry daily design optimization of turbomachinery blades. 


\section{CHAPTER I}

\section{INTRODUCTION}

Modern compressors have a wide variety of applications, e.g. refrigeration and air conditioning systems, pipeline transport of natural gas, petroleum refineries, gas turbine systems, jet engines, and in many various industrial, manufacturing and building processes. The compressor is one of the most important components within an aero engine. Compressors can vary in size from a few feet to tens of feet in diameter, depending on their application. An inherently complex high-speed flow coupled with highly loaded blades can make the efficient operation of the compression system a daunting task. In order to run the compressor efficiently, structural instabilities, excessive deformation of the structure, and flow instabilities such as stall and surge must be avoided or dealt with effectively.

The fundamental operations of a multistage axial compressor were known and presented to the French Academie des Sciences $^{1-2}$ as early as 1853. Since then the working of a compressor has been studied extensively, and compressors have evolved significantly. In order to achieve the best performance, the aerodynamic characteristics of the individual components must be fully understood. An understanding of the aerodynamic characteristics of the compression system along with the design of the system components forms the core of this study. 
The complex mechanism associated with the compressors makes its design a challenging task. Traditionally, designers have used a combination of analytical tools, extensive commercial databases, and expertise in making design decisions. This approach leads to an evolutionary approach for steering the design towards safe realizable conditions and configurations. In recent years considerable interest has been generated in the jet propulsion community in understanding the inner workings of the compressor and the design of compressors. A recent overview into single and multi-stage axial-flow compressor design may be found in References [3]-[8]. A detailed survey of current design and analysis methodologies is given in Chapter 2.

\subsection{Understanding the Compressor Operation}

The various parts of an aero engine where the compressor forms the first

component in the engine core is shown in Figure 1.1. The central purpose of a compressor is to increase the total pressure of the working fluid using shaft work. In an axial compressor, the increase in pressure occurs in two parts. Firstly the working fluid is accelerated through rotating blades (rotor), which causes an increase in the kinetic energy of the fluid. This high kinetic energy is then converted into pressure by decelerating the gas in a stationary blade passage (stator). The stator blades are required in order to ensure reasonable efficiency; by converting the energy associated with swirl into pressure. The stator places a secondary, but equally important role of aligning the flow for the following stage. Improvements can be made by replacing the stators with a second set of rotor rotating in the opposite direction, but these designs have generally proven to be too 
complex to be worthwhile. A combination of a row of rotor blades and a row of stator blades together form a compressor stage ${ }^{9}$.

Figure 1.2 shows a schematic of single stage axial compressor along with a row of stationary inlet guide vanes. Here the direction of the flow is from left to right and the direction of rotation for the rotor blades is from bottom to top. The purpose of the guide vanes is to suitably align the flow at the inlet of the rotor such that maximum flow acceleration is achieved through the rotor blade rows. On leaving the rotor exit, the flow is decelerated through the stator row and then enters the subsequent stage of the compressor.

The number of stages in a compression system varies with the application. Increasing the number of stages increases the total pressure ratio for the system, but decreases the overall efficiency and increases weight and cost of the system. The blades compress the working fluid that enters into progressively smaller volumes, resulting in an increase in the stagnation pressure and an increase in the stagnation enthalpy of the air. In gas turbine engines, the compressed air is then fed into the combustion chamber, leading to efficient combustion.

Although much of the early axial compressors dealt with subsonic flows, modern designs require the compression systems to have higher-pressure ratios and mass flow rates. Most of the axial compressors currently in use have a transonic compression system, where regions of subsonic and supersonic flow both exist in the blade passages. Supersonic flows predominantly occur near the leading edge tip. Transonic compression systems forms an integral part of the high-bypass ratio engines. The literature on 
transonic compressors is vast. Reference [9]-[10] discuss the transonic nature of compressors, and more advanced topics on transonic compressor performance may be found in References [11]-[12].

\subsection{Stability of Compression System}

Changes in the operation conditions will affect the compression system. Stability is a measure of the compression system's ability to recover from these deliberate changes, which could also be transient in occurrence. The system is considered stable if it returns to its operating point, and unstable if the disturbance drives the compressor away from the starting condition. The stable operating range for an axial compressor is limited at both very low and very high mass flow rates. In the case where the mass flow rate is high, shocks form in the system and the flow through the compressor chokes. If the mass flow rate through the compressor is low, flow instabilities will occur in the system that will lead to an inefficient operation. Clearly a compression system is subject to high variations in operating conditions; hence, stable operation is a high priority.

Figure 1.3 shows a typical performance map for the compression system where the rotor RPM is varied. The dashed line on this plot represents the stall/surge line, i.e. mass flow below which the flow instabilities occur. But stall and surge could still occur on the right side of the stall/surge line, which is why the operating points are shifted to another broken line called the surge avoidance line. The surge point on the performance map is the demarcation between the stable and unstable compressor operation. The region between the stall line and surge avoidance line serves as a safety margin. 
The closer the operating point is to the surge/stall line, the greater the benefit of increased pressure ratio, but the risk of stall or surge also increases. Hence another parallel line is drawn to the left of the stall/surge line; this is the desired extension in the operating range. A designer strives to extend the operating range of the compression system by utilizing active/passive flow control techniques or through a redesign of the blades. In this work, the latter approach (redesign) is considered in detail.

\subsection{Compressor Design Overview}

In the jet engine application, the compressor faces a wide variety of operating conditions. On the ground at takeoff the inlet pressure is high, inlet speed zero, and the compressor is spun at varying speeds to generate the required pressure rise and mass flow rate. Once in flight the inlet pressure drops, but the inlet speed increases (due to the forward motion of the aircraft) to recover some of this pressure, and the compressor tends to run at a single speed for long periods of time. Clearly, this large variation in rotor RPM and mass flow rate both need to be considered while designing an axial flow compressor.

As discussed by Lakshminarayana ${ }^{10}$ early designs transonic and supersonic compressors were failures. A combination of poor efficiencies and low reliability led to bad designs. Initially it was believed that the low efficiencies were due to the shock patterns alone. But after successive design failures it was recognized that the losses attributable to flow blockages that are caused by the shocks. Since then significant improvements have been made in blading design, shock optimization and hub-to-tip design. A brief summary of the some of the early designs is given by Hawthorne ${ }^{3}$. 
Although increasing the number of stages leads to higher overall pressure rise, it also increases the weight and length of the overall compression system. The application often dictates the allowed weight and size of the compression system, which determines the number of stages in the compression system. Each stage is smaller in diameter than the last, as the volume rate of flow of air is reduced by the compression of the preceding stage. Axial compressors therefore generally have a conical shape, widest at the inlet. Typically, modern axial compressors have anywhere between 9 and 15 stages.

Figure 1.4 illustrates the basic compressor design problem. The figure shows the main components that need to be designed, namely the geometry of the end-wall contour, and the geometry of the blade rows. Commonly used multistage compressor designs tend to perform design analysis on a mean stream surface (shown in figure 1.4) in an axisymmetric fashion. The mean stream surface is used as a baseline to obtain both the end-wall geometry and blade geometry. An overview of this type of design method may be found in $\operatorname{Ref}[4]$.

From an aerodynamic perspective, a more precise 3-D design of the compressor blade is very important as this ensures maximum blade loading. The aerodynamics of a compressor blade is also closely linked to its structural and aeroacoustic responses. An increased loading on the compressor blades can cause increased structural deformations to the blade, eventually leading to structural failure. An inefficient design of the blades can inherently lead to increased acoustic response from the blade, from rotor-stator interactions and shock patterns. Clearly, the design of the compression system is a multifaceted problem. 
In this work the structural response of the rotor to the airloads is included, while the aeroacoustic characteristics of the design are handled through an a posteriori assessment. These details will be discussed later.

\subsection{Objectives and Organization of Present Work}

This study aims to develop and demonstrate a systematic investigation to understand the impact of stage design on compressor performance- total pressure ratio and adiabatic efficiency. A methodology will be presented whereby the rotor and stator blades are designed based on a parametric description of the blade surface design variables and subsequently optimized using a multi-objective optimization technique.

This thesis is organized at follows. In Chapter II, a detailed review of existing design and first principles based modeling of axial compressor is given and the limitations of existing design approaches are identified. A set of state of the art analysis and design tools are next selected and described in Chapter III. Chapter III also discusses the present multidisciplinary formulation for compressor design using these tools. Chapter IV discusses the validation of the analysis tools that form the backbone of the present study. In Chapter V, results from preliminary application of methodology are presented where an existing compression system is redesigned taking into account only the aerodynamic aspects of the design problem. Subsequently, the design methodology is studied further and applied in a more comprehensive manner where a multiobjective

optimization is performed, results for which are presented in Chapter VI. Finally, the conclusions and recommendations for further improvements of compressor design methods are given in Chapter VII. 


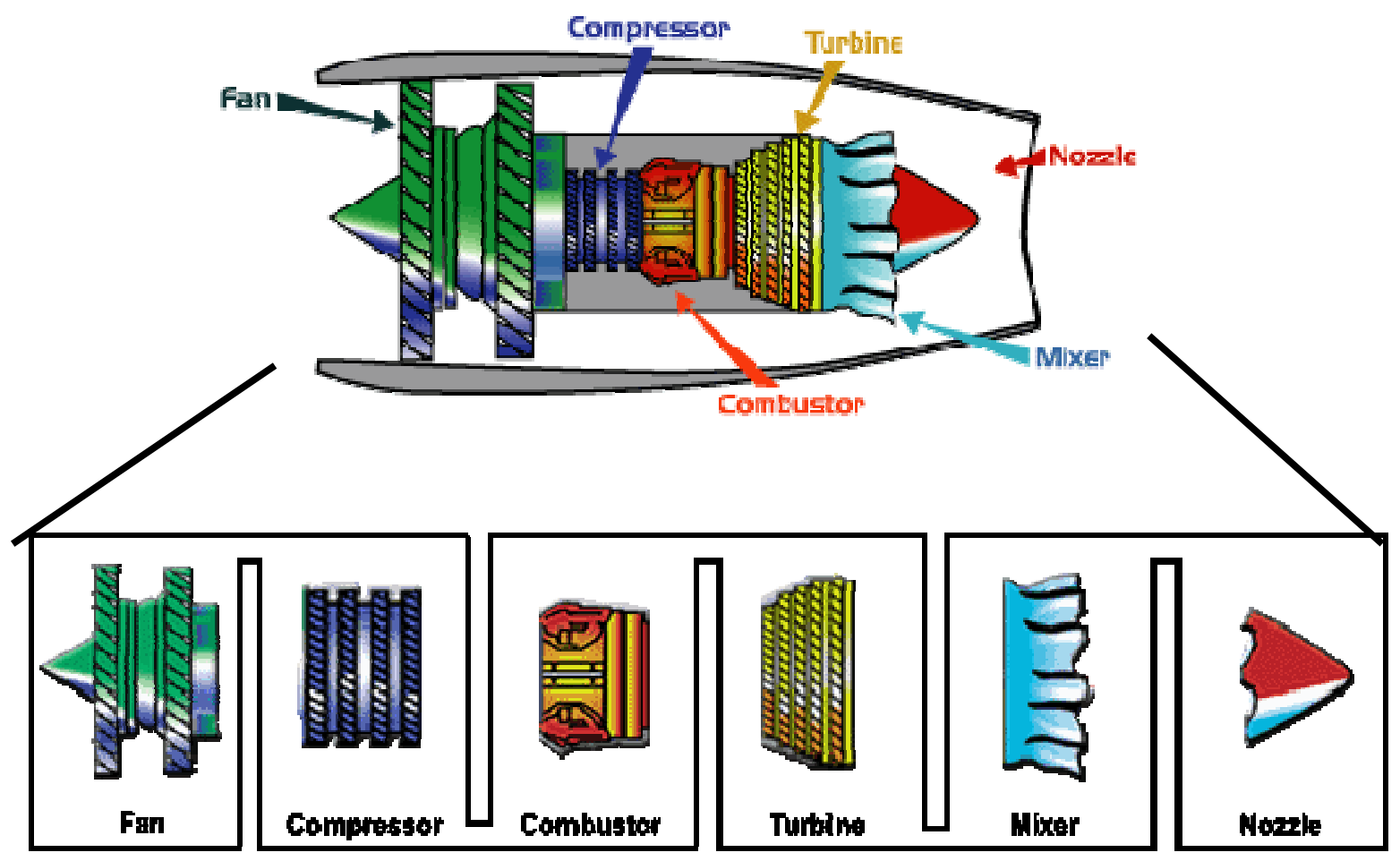

Figure 1.1: Various components of a commonly used turbo-engine, Referencehttp://en.wikipedia.org/wiki/Image:Axial-flow-compressor.png. 


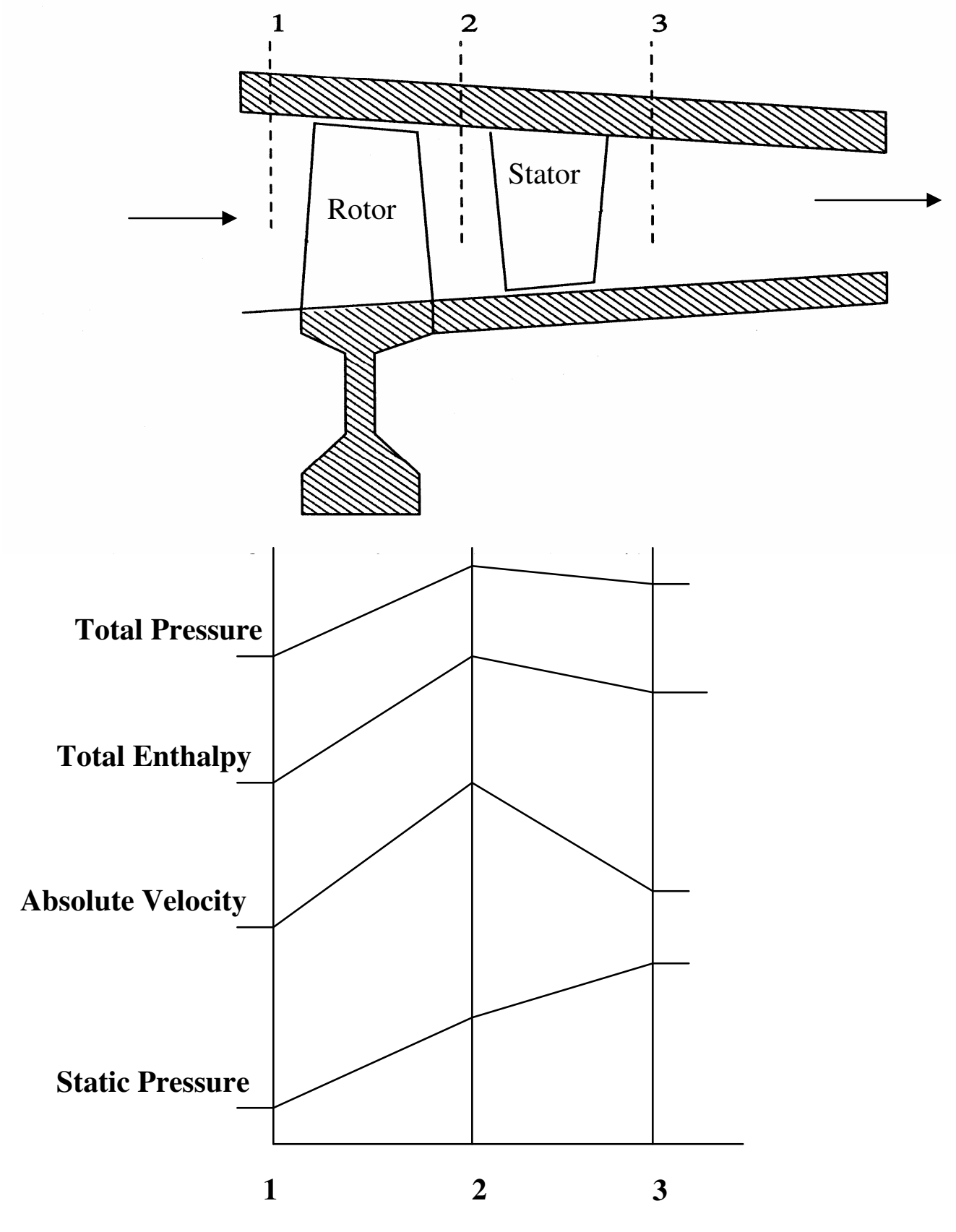

Figure 1.2: Schematic diagram of rotor-stator configuration and changes in fluid properties through a stage, Reference [1]. 


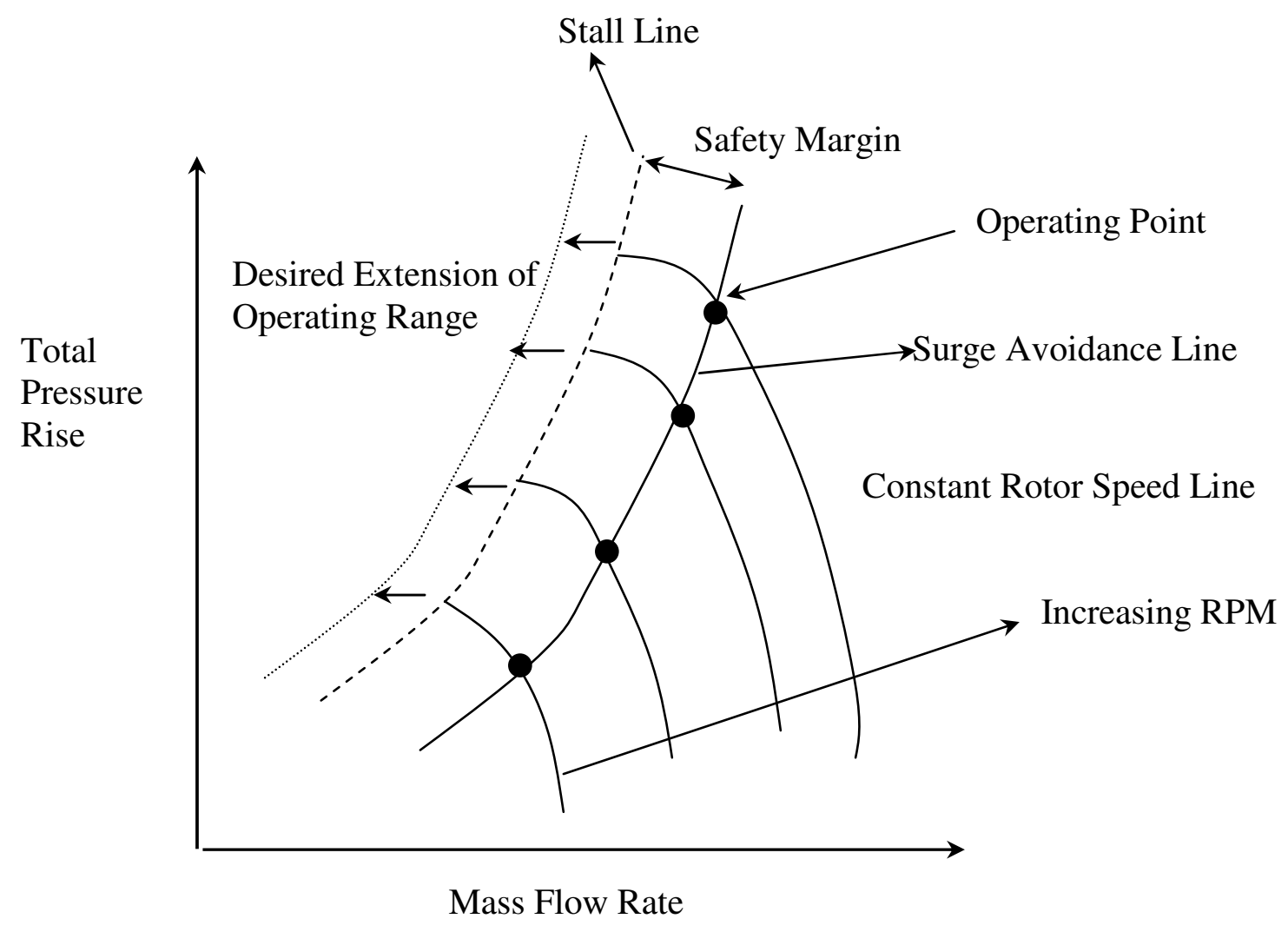

Figure 1.3: Typical performance map for an axial compressor with stability issues.

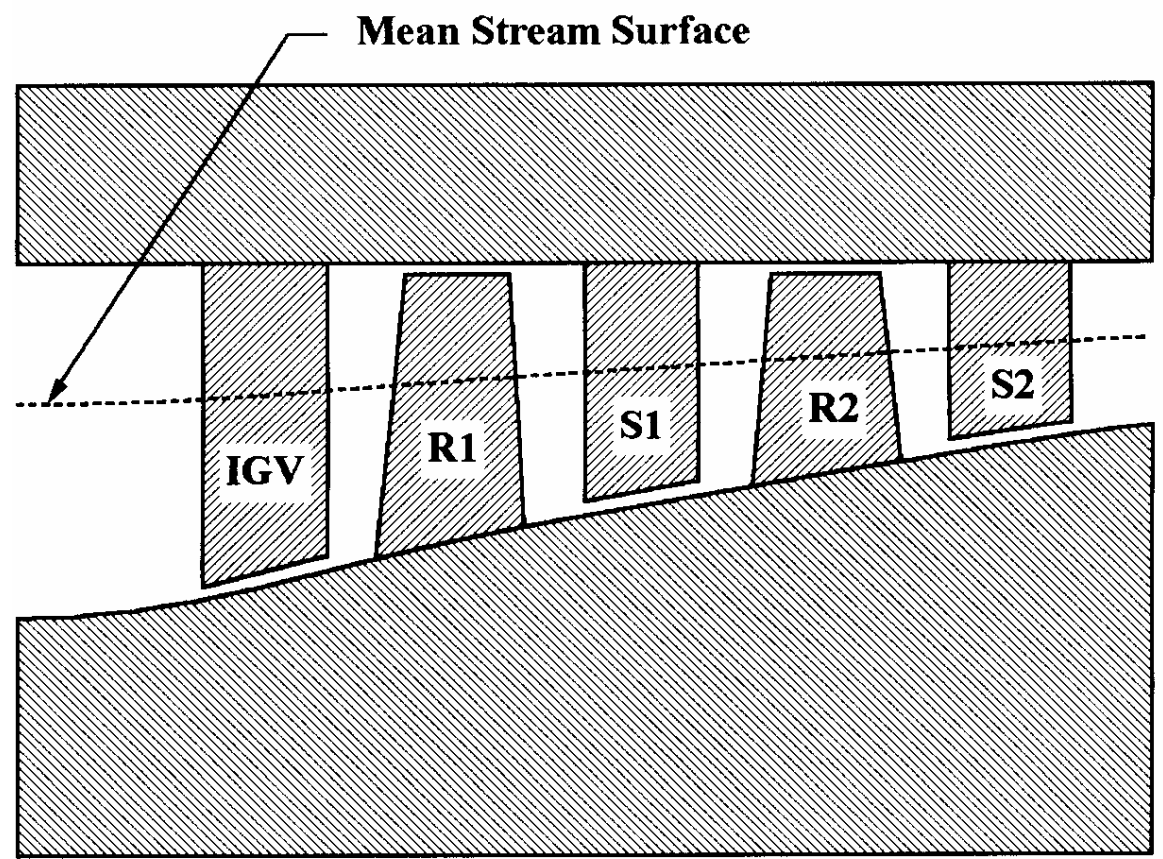

Figure 1.4: Compressor geometry design with endwalls and blade rows, Reference [5]. 


\section{CHAPTER II}

\section{DESIGN OF AXIAL FLOW COMPRESSORS}

It was indicated on the previous chapter that the design of an axial flow compressor involves many factors, and hence can times be a challenging problem. Even a small improvement in the operation of a commercial aircraft engine can have substantial benefits in yearly fuel costs. This chapter is devoted to reviewing and assessing the various methodologies presently used for design of compressors. A review of prior computational study of compressors is given in Section 2.1. Section 2.2 gives a chronological background of the methodologies used to design axial compressors. System level engine design overview is given in Section 2.3 Previously published literature on various optimization techniques is discussed in Section 2.4. In Section 2.5, a review of some of the aeroacoustic analysis carried out on axial compressor is presented. An overview of the currently proposed methodology is given in Section 2.6.

\subsection{Computational Studies on Compressor Performance}

In order to develop an accurate turbomachinery flow solver and obtain satisfactory results careful attention needs to be paid to the viscous terms, artificial viscosity, turbulence modeling, and grid resolution. Early turbomachinery performance

predictions using CFD were mostly done in two dimensions. Such cascade analyses for 
both compressors and turbines were performed by many researchers ${ }^{13-16}$. Flow in an axial flow compressor can be unsteady and vortical by nature ${ }^{17-20}$ and hence accurate modeling of the viscous effects and turbulence is imperative.

Over the past two decades much work has been done in developing phenomenological numerical methods to simulate and understand the flow physics in turbomachines. During this time the field of computational fluid dynamics has undergone significant changes, providing designers and engineers with a useful capability to model and study the inherently three-dimensional flows in compressor. Recently with the increased computing power and developments in post-processing and visualization tools, accurate three dimensional simulations of rotary machines are now achievable. Also, with the availability of reliable experimental test data by $\mathrm{AGARD}^{21}$, researchers now have access to a plethora of data for validation of turbomachinery codes. Since then a number of three dimensional CFD codes capable of analyzing single and multiple blade passage turbomachinery flow have been developed by many researchers ${ }^{22-29}$.

Most of aforementioned three dimensional computational methods solve isolated turbomachinery rows, i.e. either the rotor or the stator in isolation and are commonly used for turbomachinery design. However, it is worth noting that except for some fans and pumps, few turbomachines operate as isolated blade rows. Most turbomachines include at least a stator, the purpose of which is to add or remove swirl, and often include many stages to do more work. Computational codes capable of analyzing multistage turbomachinery configurations have been developed by Denton ${ }^{30}$, Hall ${ }^{31}$ and Chima ${ }^{32}$. Recently an isolated blade row three dimensional compressible unsteady flow solver 
initially developed by Niazi et al. ${ }^{33}$ was successfully modified to include multistage capabilities by Zaki et $\mathrm{al}^{34}$.

\subsection{Background of Compressor Design Methods}

Blade design methods can be classified broadly into two approaches, inverse and direct approaches. In inverse design methods, desirable flow features on the blade, such as pressure distribution and/or pressure loading distribution are specified and the blade geometry is computed in such way that the specified flow features are produced. Typically the difference in static pressure across the blade and the normal thickness distribution is specified and an analysis of the flow field around the initial blade geometry is carried out. Next, the new blade geometry is calculated and the corresponding computational mesh is generated. The process is typically repeated until a blade geometry that results in the desired flow features is obtained. If done efficiently, this design method can be applied successfully.

In the direct approach the blade geometry is analyzed directly by a CFD analysis and/or experiments. In this method either the parameters that directly influence the blade section are modified or the $(\mathrm{x}, \mathrm{y}, \mathrm{z})$ coordinates of the existing blade geometry is altered. The geometry is subsequently analyzed and the influence of design variables on its overall performance is assessed.

In the late 1940s and early 1950s, axial compressor and turbine design substantially relied on empirical correlations of data. For axial compressors these data came from cascade tests of blades where the profile was usually prescribed, usually $\mathrm{C} 4$, C5, NACA series, coupled with some simple analytical methods ${ }^{35}$. Howell ${ }^{36}$, and Carter 
et $\mathrm{al}^{37}$ performed mean line design of axial compressors that relied on cascade data for flow deviation. Cascade data also provided information on profiles and secondary losses. Most of these studies were performed for incompressible flow and provided limited understanding of the physics of the flow. In parallel early work on inverse design was performed by $\mathrm{Stanitz}^{38}$ on turbine blades. Stanitz developed the prescribed velocity distribution method (PVD) to obtain the required blade shape once the surface velocity was prescribed.

Towards the end of the 1950s and the beginning of the 1960s advances in blade to blade flows, secondary flows, through-flow methods and clearance flows had great impact on practical compressor design approaches. Direct design problems of the twodimensional potential flow in given compressor and turbine were developed. Gostelow ${ }^{39}$ for incompressible flow and Hobson ${ }^{40}$ for compressible flow provided exact solutions for specific blade profiles. They also developed a methodology to allow for boundary layer growth on the blade surfaces. Hawthorne ${ }^{41}$ and $\mathrm{Smith}^{42}$ made significant progress in an effort to understand the fluid mechanics of secondary flow. However they were unsuccessful in integrating this work into the design methods; the clearance losses and problems in secondary flow still persisted. In later 1960s pioneering work on clearance flows was performed by Lakshminarayana ${ }^{43-44}$; this work gave information on how blade lift varied along with the blade span and on the magnitude of the tip clearance loss.

Early inverse design methods were developed in parallel by Katsanis ${ }^{45}$ (19611967). The method developed by Katsanis provided the capability to analyze the two dimensional, incompressible, ideal flow problems for an infinite cascade of blades, or 
equivalently, a two-dimensional, circular cascade of constant radius, as in an axial-flow turbine.

In the 1970 s with the advent of techniques such as Murman and Cole method ${ }^{46}$ and Jameson's rotated difference scheme ${ }^{47}$ it became possible to solve transonic potential flow equations for a two dimensional airfoils and later three-dimensional wings. These advancements had significant impact on airfoil and wing design. It also had a great impact on compressor design as the advanced schemes could be coupled with the previously developed compressor potential flow solvers.

These transonic methods found their way into inverse design methods that were being investigated by Dulikravich ${ }^{48}$ (1980) and Meauze ${ }^{49}$ (1982). Dulikravich developed CAS22, a program capable of designing shock free airfoil cascades and applicable to aerodynamic analysis and transonic shock free redesign of existing two-dimensional cascades of airfoils. Some of the other application of two-dimensional inverse design method was done by Meauze. Later in 1995, Dulikravich ${ }^{50}$ extended the two-dimensional inverse cascade design methodology to three-dimensional blades.

In the 1990s with the significant developments in CFD, three-dimensional design and analysis of axial compressor configurations has been made possible. Bolger and Horlock $^{51}$ performed direct design analysis on a four-stage compressor and showed that the repeating stage phenomenon where the blockages increased across each row. By this time direct design concepts such as the use of bowed blades to control secondary loss in turbines and the use of sweep and bow to reduce corner separations in compressors were introduced. $\mathrm{Hah}^{52-53}$, Wadia ${ }^{54}$, Denton and $\mathrm{Xu}^{55}$ carried out the pioneering work on the 
global effects of sweep and lean on transonic fan performance. They found that the swept blade could effectively improve the aerodynamic performance of compressors.

In parallel, three-dimensional axial compressor inverse design and analysis was also evolving. In 1993 Dang $^{56}$ developed a full three-dimensional inverse design method for turbomachinery blades in transonic flow. Then in 1997, Dang ${ }^{57}$ et al presented a three-dimensional inverse design in which they redesigned transonic fan NASA 67 configuration. Zangeneh ${ }^{58}$ also developed a three dimensional inverse design method applicable to radial and mixed-flow Turbomachines. Zanganeh method has subsequently been updated by $\mathrm{Hu}^{59}$ et al. using inverse design code TURBOdesign- 2 and applied it NASA 67 and NASA 37 configurations, to arrive at improved configurations. In another study, Keshin ${ }^{60}$ et al. developed an automated multi-objective optimization strategy to find best blade section geometries with respect to loss and working range.

Recently, the use of sweep, lean (dihedral) and skew (stacking line in rotational direction) in axial flow compressor rotor has become a matter of interest in direct design of turbomachinery blades. The philosophy behind the sweep effect is the same as that on a swept aircraft wing, i.e. to make the shock swept relative to the incident flow. By doing this the component of the Mach number perpendicular to the shock, and hence the shock loss, is reduced. Sweep corresponds to moving the blade in the axial direction, whereas lean corresponds to moving the blade in the circumferential direction. Many researchers ${ }^{61-63}$ have applied the sweep and/or lean concept to single stage and multistage configurations and found beneficial results. 
Both the inverse and direct design methods discussed earlier have their advantages and disadvantages. For example, the direct method can sometimes be trialand-error, especially when the design parameters are selected in an ad-hoc manner. But it has its advantages over the inverse design method, which usually requires a number of inputs, some of which are not always known (e.g. 3-D pressure distribution) so as to produce the desired flow features. Hence, the direct method, which generally does not need such detailed knowledge of the flow, is usually preferred.

The direct designs described earlier make use of techniques such as sweeping the blade and leaning the blade in order to carry out design optimization. Although these methods are very effective, they do not perform basic blade parameterization, wherein the blade sections are rebuilt based on blade camber line and other parameters which affect the blade section. In order to consistently rebuild a three-dimensional compressor blade, the blade camber lines, thickness distributions and the blade stack line need to be approximated as functions of several key parameters. Since these parameters directly influence the blade section, it is more useful to use these parameters as design variables rather than sweeping or leaning existing blade geometry.

In the early parametric design methods developed by Dunham ${ }^{64}$, the thickness around a camber line was specified. However differences in the nature of the physical phenomenon on the suction and pressure sides of the airfoil promoted the development of methods that independently controlled each surface. More recently, researchers have showed the importance of the airfoil curvature distribution on its performance and have described potential methods that can be used to design highly differentiable blade 
surfaces $^{65-67}$. Corral and Pastor ${ }^{68}$ developed a set of chained $\mathrm{G}^{3}$ Bezier polynomials capable of generating turbomachinery airfoils.

\subsection{System Level Engine Design Overview}

This study performs design analysis on an axial compressor at the component level whereby the compressor blades are studied. But design studies on turbomachinery configurations can also be performed at an overall engine system level where the overall operation of the engine is the focus rather than individual components. Such system level design studies have been extensively performed at the Aerospace Systems Design Laboratory (ASDL) at Georgia Tech. A probabilistic design methodology for commercial aircraft engine cycle selection was proposed by Marvis ${ }^{69}$ et al. An evaluation of a lost thrust method for analysis of thermodynamic performance in gas turbine engines has

been investigated by $\operatorname{Roth}^{70}$. Another study on how probabilistic methods can be utilized to rationally and analytically make design decisions in the presence of uncertainty, with emphasis on the use of probabilistic sensitivities in the aircraft gas turbine engine preliminary design process was done by Marvis ${ }^{71}$. Other references on system level engine design can be found in References [72-73].

\subsection{Literature on Optimization Techniques}

Numerical optimization techniques have been successfully used for a variety of design problems. The aerodynamic design optimization of a blade itself is a challenging 
problem. Firstly, the performance of a transonic blade is very sensitive to its shape and hence the blade shape must be parameterized with the appropriate number of parameters to be optimized. In addition, the objective function setting of an aerodynamic design optimization problem is often multidimensional and non-linear because the flow field is governed by a system of non-linear partial differential equations. And finally, constraints such as operating mass flow range, noise characteristics and others make the aerodynamics blade shape optimization a valid problem. Many optimization algorithmssuch as gradient based method, orthogonal array method, response surface method (RSM), artificial intelligence methods such as neural network and evolutionary algorithms (EA's) have been reported with varying degree of success.

The gradient-based method is a well-known optimization algorithm in which the optimum is probed by calculating the local gradient information. The method searches for the direction in which the descent slope for the output variable is the steepest and proceeds iteratively until a new local minimum is encountered. CONMIN ( Minimization) - developed by Vanderplaats ${ }^{74}$ uses such a gradient based approach and has been actively used for airfoil design. The gradient based methods have been widely used for aerodynamic design such as wing design ${ }^{75}$ and supersonic wing-body designs ${ }^{76}$. Recently this method has been applied to turbomachinery blade optimization problems; Lee et $\mathrm{al}^{77}$ applied it to a vaneless diffuser in a centrifugal compressor and Koller et $\mathrm{al}^{78}$ used it to design compressor airfoils.

Evolutionary algorithms (EAs) are another type of optimization algorithm, they mimic the natural process of the evolution of genes, where a biological population 
evolves over generations to stochastically search for the optimal value. One of the key features of EAs is its ability to simultaneously search from multiple points in the design space, instead of the single point approach that is used in gradient-based method. EAs have been successfully applied to turbomachinery blade shape optimization problems by $\mathrm{Yi}^{79}$, Oyama $^{80}$, and Uelschen ${ }^{81}$.

Another way to perform the aerodynamic design optimization on a blade is by the Response Surface Methodology (RSM). Originally, the response surface methods were developed to analyze experimental data and to create empirical models of the response values that were observed. A response surface is a smooth analytic function that represents the true relationship between the input (design variables) and the output (expected responses). Optimization using RSM is a series of statistical and mathematical processes. Firstly, data is generated by numerical computations (or experiments). Then, a response surface is constructed by interpolating the data. And finally, optimization of the objective function on the surface is performed. Recently RSM has been successful implemented for turbomachinery blade optimization. $\mathrm{Ahn}^{82}$ et al carried out the optimization on the stacking line of axial compressor rotor blades. In 2006, Jang ${ }^{83}$ used RSM to perform shape optimization to a stator blade in a single-stage transonic axial compressor. They found an increase in adiabatic efficiency due to the suppression of flow separation on the suction surface of the blade.

With the objective functions being multimodal, it is possible that the gradientbased search will only be able to locate the local minimum and not necessarily the global minimum. A repetitive search might be required in order to consistently check and 
subsequently obtain the global minima. Due to this drawback, the gradient-based method is neither efficient nor robust. Although EAs are fairly robust once the algorithm is efficiently designed, the actual design of the evolutionary algorithm can at times be intricate. Depending on its application, the EAs range from being very simple to develop to extremely complex to generate. For the aerodynamic design optimization of a compressor stage such as the one proposed in this study, use of the evolutionary algorithm for optimization can prove to be the convoluted approach. On the other hand, RSM is relatively easy to generate once the design functions are identified. RSM's ability to smooth out high frequency noise of the objective function makes it a viable choice. It also provides us with an extremely robust tool capable of efficiently integrating in the overall design environment.

\subsection{Overview of Compressor Aeroacoustics Techniques}

Compressor noise constitutes a serious problem in connection with the operation of commercial jet-powered aircraft. Recently, this problem has been aggravated by the conversion to a turbofan type of power plant, where the compressor and fan components are more powerful. One of the main factors causing the noise to be annoying is the presence of discrete frequency components associated with rotating machinery. More often than not, these discrete frequency components are at frequencies that are very

sensitive to the ear. Noise levels from compressors are reported ${ }^{84}$ to be more serious at landing approach than during take-off. Even though engines are operating at partial power during landing approach, the aircraft, is closer to people on the ground. 
The compressor noise problem can be conveniently considered in two parts; namely, tonal noise generated by the rotating machinery, and the random noise generated due to the pressure fluctuations. For the discrete tone generation, there are fundamentally three mechanisms of generation. Firstly, when the rotor is in isolation, a discrete tone is propagated as a result of the rotating pressure fields associated with each blade being sensed as a cyclic fluctuation. When operating at supersonic conditions, this effect can commonly result in a series of apparently discordant tones between the rotor and the blade passing frequencies. The tones are caused by variations in amplitude and direction of the various pressure waves.

More often than not in a typical engine stage, the rotating and stationary blade system usually have very little space between each other. Here a second source of discrete noise is generated as a result of the interaction between the pressure fields around the rotor and the stator blade rows. But this source mechanism can be easily destroyed by increasing the spacing between the two stages. Under such circumstances, a third mechanism of generation can exist. Here, the downstream stage senses changes in incidence at its leading edge due to the passage through the wakes from the upstream stage. Sometimes changes in the incidence angles can be cyclic in nature, hence producing a discrete tone and its harmonics that correspond to the number of blades and the speed or rotation of the unit. Suppression of this interaction mechanism is possible by minimizing the pressure and turbulence fluctuations within the wake.

Two basic mechanisms exist for random noise generation. The first is as a result of the noise generated by a blade operating in a non-turbulent environment from pressure 
fluctuations on its surface. These pressure fluctuations are caused by the eddy shedding at the trailing edge. The second mechanism occurs when significant upstream turbulence exists, as on all other blades in the fan or compressor. The blade senses this turbulence as changes in incidence, which effects the pressure fluctuations on its surface, again resulting in random noise generation.

Most of the early turbomachinery related aeroacoustic work had been focused on fan noise, as it is one of the major noise components- fan-exhaust during take-off and fan-inlet during landing. Rao $^{85}$ and Heidelberg ${ }^{86}$ have performed fan noise control using sweep/lean and found beneficial results. Some of the early studies on axial flow compressor noise were done by Tyler and $\operatorname{Sorfin}^{87}$. Since then noise control on axial flow compressors has become an actively researched topic. Schulten ${ }^{88}$ investigated the sound generated by rotor wakes interacting with a leaned vaned stator.

Methods such as leaning and sweeping compressor blades have been commonly utilized for compressor noise reduction. Envia ${ }^{89-90}$ investigated the influence of vane sweep on rotor-stator interaction noise. A conclusion of particular importance found in that study was that orientation of the vane sweep must be chosen to enhance the natural phase lag caused by wake lean, in which case rather small sweep angles substantially reduce the noise levels. Huff ${ }^{91}$ et al. developed a theoretical model to quantify the benefits of stator vane sweep and lean in reducing rotor-stator interaction tone fan noise. An optimal vane sweep and lean combination for minimizing tone noise emissions was reported in that study. Recently Elhadidi ${ }^{92}$ studied passive-noise control by blade lean 
and sweep. They showed that vane lean and sweep could be used effectively for certain design configurations to reduce the radiated sound power.

It is noteworthy that the aero-acoustic studies mentioned above were either performed experimentally or by using theoretical/semi-empirical models. Although most of the aforementioned methods are extremely elaborate, they do not take into account important effects from the inherently three-dimensional nature of the flow. Nowadays more attention is focused on CFD as a method for predicting noise generated by turbomachinery. In fact, three-dimensional CFD models might help overcome some of the limits of the semi-empirical models. Compressor rotor wake and tone noise caused by rotor wake/stator interactions was studied by Dawes ${ }^{93}$ using CFD. It was found that deep wakes with small widths generate less acoustic power and low harmonics.

Another observation about the previously mentioned aeroacoustic studies would be that they were performed solely for noise benefits. Although aerodynamics and acoustics are related, it is not necessary that noise benefits result in improvements in blade aerodynamics. Hence a need still exists to couple blade aerodynamics directly with noise characteristics from compressor blades. Intuitively speaking, an aerodynamically optimized compressor blade should result in reduced noise levels, but this is not always the case and hence needs careful consideration. The intention would be to minimize the blade noise while preserving the aerodynamic benefits via design optimization. 


\subsection{Current Design Methodology}

Most of the aforementioned parametric design methodologies were initially conceived for design turbine blades, and therefore characteristics of these approaches are oriented more towards solution of turbine blade issues. Although some of the formulation can be extended to design other aerodynamic shapes, a need for parametric design of compressor blades still exists. This study aims to develop a methodology where by the blades are designed parametrically and subsequently optimized for certain objective functions. The study is multifaceted, one that incorporates high fidelity computational fluid dynamics and design tools such as design of experiments and response surface methods. A low order coupling involving one dimensional flow analysis and design tools have previously been performed with certain success, but a need for higher order flow analysis coupled with design tools for optimization of compressor stage still exists.

In the first part of the study, a preliminary application of the methodology will be performed and design variables will be selected based on engineering based intuition. In the first part the stage blades optimization is based purely on attaining aerodynamic benefits. In the second part of the study the design variables are screened by examining the effects of each design variable on the response(s). In this case multi-objective optimization will be used to couple the aerodynamic responses to the aeroacoustic responses and obtain an optimized configuration. In both cases a comprehensive three dimensional flow solver is used to examine the flow in the compressor. Figure 2.1 shows box path of the proposed methodology and figure 2.2 shows a flowchart describing the same methodology. 


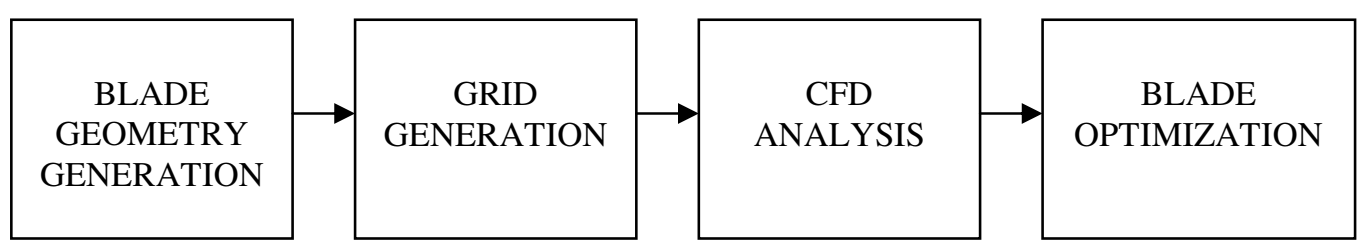

Figure 2.1: Box diagram showing the proposed methodology.

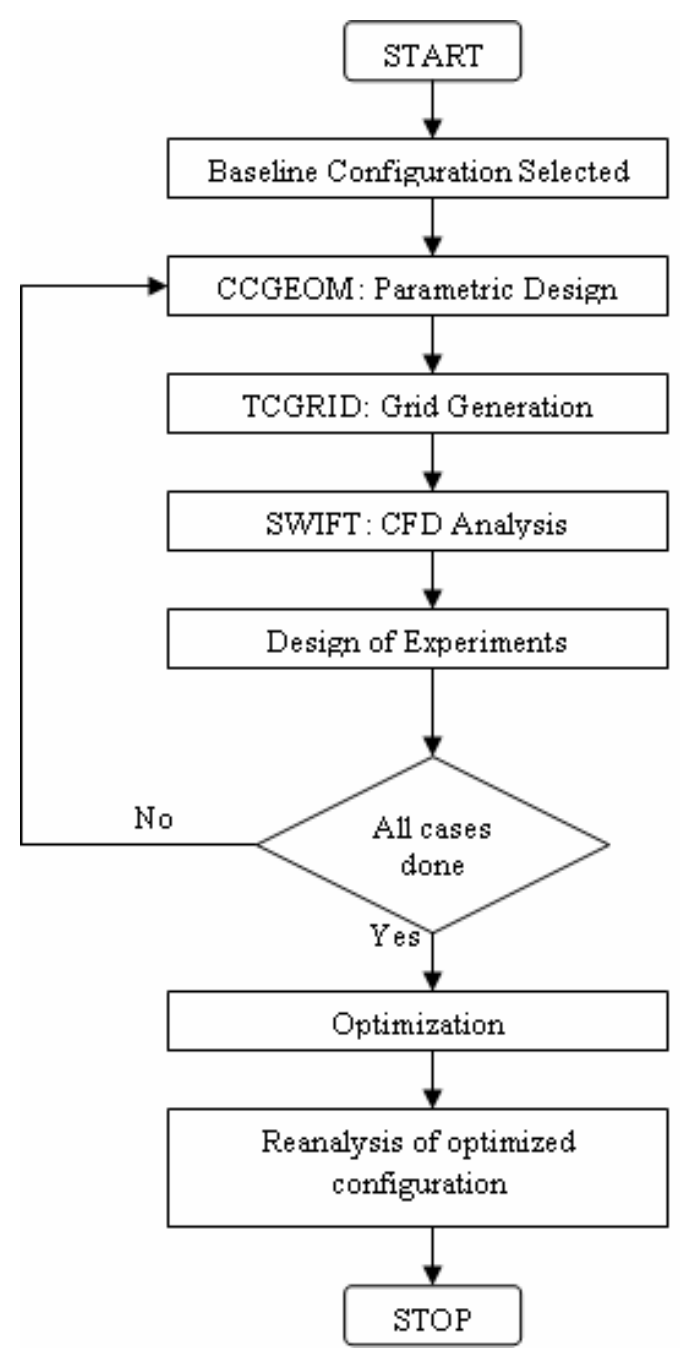

Figure 2.2: Flowchart showing the sequence of events in the proposed design methodology. 


\section{CHAPTER III}

\section{MATHEMATICAL FORMULATION AND TOOLS}

In order to study compressor flow details, solution of the 3-D Navier-Stokes equations is necessitated. Previously used analytical solutions are only valid to simple flows and configurations and therefore, numerical techniques are needed for more complex problems. In an effort to accurately model the flow within a compression system in this study, a very robust flow solver developed by Chima ${ }^{94}$ is used.

In section 3.1 the governing equations are presented. The initial conditions are discussed in section 3.2. The boundary conditions used for the computational analysis are discussed in section 3.3. In Section 3.4 the time marching approach is presented. The turbulence modeling method implemented in this work is discussed in section 3.5. In section 3.6 the grid generation technique is discussed. Details on the various design tools used are present in section 3.7. Section 3.8 gives details on the total pressure ratio and adiabatic efficiency calculations. The aeroacoustic method implemented is described in section 3.9. The structural analysis adopted for the study is discussed in section 3.10. Finally, the method for the selection of the design variables is presented in section 3.11. 


\subsection{Governing Equations}

The Navier-Stokes equations describe the conservation of mass, momentum, and energy. The fluid under consideration in this study is air, for which the Newtonian fluid assumption is a valid approximation. The Navier-Stokes equations are written in a Cartesian $(\mathrm{x}, \mathrm{y}, \mathrm{z})$ coordinate system rotating with angular velocity $\Omega$ about the $\mathrm{x}$-axis. The rotation introduces source terms in the $\mathrm{y}$ and $\mathrm{z}$ momentum equations. Under the assumption of no body forces, no external heat addition, and no mass diffusion, the 3-D Navier-Stokes equations may be written in the following vector form in the Cartesian system:

$$
\frac{\partial \vec{q}}{\partial t}+\frac{\partial \vec{F}_{I}}{\partial x}+\frac{\partial \vec{G}_{I}}{\partial y}+\frac{\partial \vec{H}_{I}}{\partial z}=\frac{\partial \vec{F}_{V}}{\partial x}+\frac{\partial \vec{G}_{V}}{\partial y}+\frac{\partial \vec{H}_{V}}{\partial z}
$$

$$
\vec{F}_{I}=\left\{\begin{array}{c}
\rho \\
\rho u^{2}+p \\
\rho u v \\
\rho u w \\
u(e+p)
\end{array}\right\} ; \vec{G}_{I}=\left\{\begin{array}{c}
\rho \\
\rho u v \\
\rho v^{2}+p \\
\rho v w \\
v(e+p)
\end{array}\right\}, \vec{H}_{I}=\left\{\begin{array}{c}
\rho \\
\rho u w \\
\rho v w \\
\rho w^{2}+p \\
w(e+p)
\end{array}\right\}
$$

$$
\vec{F}_{V}=\left\{\begin{array}{c}
0 \\
\tau_{x x} \\
\tau_{y x} \\
\tau_{z x} \\
E_{x}
\end{array}\right\} ; \vec{G}_{V}=\left\{\begin{array}{c}
0 \\
\tau_{x y} \\
\tau_{y y} \\
\tau_{z y} \\
E_{y}
\end{array}\right\}, \vec{H}_{V}=\left\{\begin{array}{c}
0 \\
\tau_{x z} \\
\tau_{y z} \\
\tau_{z z} \\
E_{z}
\end{array}\right\}
$$

The pressure property ' $\mathrm{p}$ ' is related to the internal energy and velocity as follows: 


$$
\begin{aligned}
& p=\rho R T \\
& p=(\gamma-1)\left[e-\frac{1}{2} \rho\left(u^{2}+v^{2}+w^{2}\right)\right]
\end{aligned}
$$

In Equation $3.3 \tau_{x x}, \tau_{x y}$ etc. are the components of the stress tensor, defined using the Stokes relations and the Boussinesq hypothesis:

$$
\left.\begin{array}{l}
\tau_{x x}=(\lambda+2 \mu) u_{x}+\lambda v_{y}+\lambda w_{z} \\
\tau_{x y}=\mu\left(u_{y}+v_{x}\right) \\
\tau_{x z}=\mu\left(u_{z}+w_{x}\right) \\
\tau_{y y}=\lambda u_{x}+\mu(\lambda+2 \mu) v_{y}+\lambda w_{z} \\
\tau_{y z}=\mu\left(v_{z}+w_{y}\right) \\
\tau_{z z}=\lambda u_{x}+\lambda v_{y}+(\lambda+2 \mu) w_{z}
\end{array}\right\}
$$

where, $\lambda$ is the bulk velocity given by $\lambda=-\frac{2}{3} \mu$ according to Stokes' Hypothesis. Also, $K$ is the conductivity related to the viscosity through the Prandtl number $(\operatorname{Pr})$ by $\operatorname{Pr}=\frac{\mu C_{P}}{K}$.

The Cartesian equations are mapped from the physical coordinate system $(\mathrm{x}, \mathrm{y}, \mathrm{z}$, t) to a general body-fitted coordinate $\operatorname{system}(\xi, \eta, \zeta, \tau)$, where transformation is expressed as follows: 


$$
\begin{aligned}
& \tau=\tau(t) \\
& \xi=\xi(x, y, z, t) \\
& \eta=\eta(x, y, z, t) \\
& \zeta=\zeta(x, y, z, t)
\end{aligned}
$$

In the $\mathrm{C}$-grids used around the blade, $\xi$-coordinate roughly follows the flow, the $\eta$-coordinate runs blade-to-blade, and the $\zeta$-coordinate runs in the spanwise direction. Thin-layer approximation is invoked to drop all the viscous derivatives in the $\xi$ direction $^{95}$. Viscous terms in the cross-channel $(\eta, \zeta)$ plane are retained. The governing equation (Equation 3.1) can now be transformed and rewritten as follows:

$$
\frac{\partial}{\partial t}\left(\frac{q}{J}\right)+\left[\partial_{\xi} \hat{E}+\partial_{\eta} \hat{F}+\partial_{\zeta} \hat{G}-\operatorname{Re}^{-1}\left(\partial_{\eta} \hat{F}_{V}+\partial_{\zeta} \hat{G}_{V}\right)\right]=\frac{H}{J}
$$

If there is no deformation of the grid from time to time step, and if the grid is rotating in a rigid fashion, then $J$ is not a function of time and maybe brought outside the time derivative. Equation 3.9i can now be rewritten as:

$$
\partial_{t} q+J\left[\partial_{\xi} \hat{E}+\partial_{\eta} \hat{F}+\partial_{\zeta} \hat{G}-\operatorname{Re}^{-1}\left(\partial_{\eta} \hat{F}_{V}+\partial_{\zeta} \hat{G}_{V}\right)\right]=H
$$

where, 


$$
\begin{aligned}
& q=\left[\begin{array}{c}
\rho \\
\rho u \\
\rho v \\
\rho w \\
e
\end{array}\right] ; H=\Omega\left[\begin{array}{c}
0 \\
0 \\
-\rho w \\
\rho v \\
0
\end{array}\right] \\
& \left.\hat{E}=J^{-1}\left[\begin{array}{c}
\rho U^{\prime} \\
\rho u U^{\prime}+\xi_{x} p \\
\rho v U^{\prime}+\xi_{y} p \\
\rho w U^{\prime}+\xi_{z} p \\
e U^{\prime}+p U
\end{array}\right] ; \hat{F}=J^{-1}\left[\begin{array}{c}
\rho V^{\prime} \\
\rho u V^{\prime}+\eta_{x} p \\
\rho v V^{\prime}+\eta_{y} p \\
\rho w V^{\prime}+\eta_{z} p \\
e V^{\prime}+p V
\end{array}\right] ; \hat{G}=J^{-1}\left[\begin{array}{c}
\rho W^{\prime} \\
\rho u W^{\prime}+\zeta_{x} p \\
\rho v W^{\prime}+\zeta_{y} p \\
\rho w W^{\prime}+\zeta_{z} p \\
e W^{\prime}+p W
\end{array}\right]\right\}
\end{aligned}
$$

These equations are written in a coordinate system that rotates with the blade. The absolute velocity component $\mathrm{u}, \mathrm{v}$ and $\mathrm{w}$, correspond to the $\mathrm{x}, \mathrm{y}$, and $\mathrm{z}$ coordinate directions, respectively. The relative velocity components $u^{\prime}, v^{\prime}$ and $w^{\prime}$ are defined with respect to the same rotating coordinate system by subtracting the appropriate components of the blade speed from the absolute velocities, giving

$$
\begin{aligned}
& u^{\prime}=u \\
& v^{\prime}=v-\Omega z \\
& w^{\prime}=w+\Omega y
\end{aligned}
$$

The relative contravariant velocity components are given by

$$
\begin{aligned}
& U^{\prime}=\xi_{x} u+\xi_{y} v^{\prime}+\xi_{z} w^{\prime} \\
& V^{\prime}=\eta_{x} u+\eta_{y} v^{\prime}+\eta_{z} w^{\prime} \\
& W^{\prime}=\zeta_{x} u+\zeta_{y} v^{\prime}+\zeta_{z} w^{\prime}
\end{aligned}
$$

Note that although $u^{\prime}=u, U^{\prime} \neq U$. The energy and static pressure are given by 3.13 and 3.14 respectively, 


$$
\begin{aligned}
& e=\rho\left[C_{v} T+\frac{1}{2} \rho\left(u^{2}+v^{2}+w^{2}\right)\right] \\
& p=(\gamma-1)\left[e-\frac{1}{2} \rho\left(u^{2}+v^{2}+w^{2}\right)\right]
\end{aligned}
$$

Using Stokes' hypothesis, the viscous flux $\hat{F}_{V}$ can be written as follows:

$$
\hat{F}_{V}=J^{-1} \mu\left[0, F_{2}, F_{3}, F_{4}, F_{5}\right]^{T}
$$

where,

$$
\left.\begin{array}{l}
F_{2}=C_{1} \partial_{\eta} u+C_{2} \eta_{x}+C_{3} \partial_{\zeta} u-C_{4} \eta_{x}+C_{5} \zeta_{x} \\
F_{3}=C_{1} \partial_{\eta} v+C_{2} \eta_{y}+C_{3} \partial_{\zeta} v-C_{4} \eta_{y}+C_{5} \zeta_{y} \\
F_{4}=C_{1} \partial_{\eta} w+C_{2} \eta_{z}+C_{3} \partial_{\zeta} w-C_{4} \eta_{z}+C_{5} \zeta_{z} \\
F_{5}=\frac{\mu \gamma}{\operatorname{Pr}}\left(C_{1} \partial_{\eta}\left(C_{V} T\right)+C_{3} \partial_{\zeta}\left(C_{V} T\right)\right)+u F_{2}+v F_{3}+w F_{4}
\end{array}\right\}
$$

and

$$
\left.\begin{array}{l}
C_{1}=\eta_{x}^{2}+\eta_{y}^{2}+\eta_{z}^{2} \\
C_{2}=\frac{1}{3}\left(\eta_{x} \partial_{\eta} u+\eta_{y} \partial_{\eta} v+\eta_{z} \partial_{\eta} w\right) \\
C_{3}=\eta_{x} \zeta_{x}+\eta_{y} \zeta_{y}+\eta_{z} \zeta_{z} \\
C_{4}=\frac{2}{3}\left(\zeta_{x} \partial_{\zeta} u+\zeta_{y} \partial_{\zeta} v+\zeta_{z} \partial_{\zeta} w\right) \\
C_{5}=\eta_{x} \partial_{\zeta} u+\eta_{y} \partial_{\zeta} v+\eta_{z} \partial_{\zeta} w
\end{array}\right\}
$$

The metric terms are defined using the following relations.

$$
\begin{gathered}
{\left[\begin{array}{lll}
\xi_{x} & \eta_{x} & \zeta_{x} \\
\xi_{y} & \eta_{y} & \zeta_{z} \\
\xi_{z} & \eta_{z} & \zeta_{z}
\end{array}\right]=J\left[\begin{array}{lll}
y_{\eta} z_{\zeta}-y_{\zeta} z_{\eta} & y_{\zeta} z_{\xi}-y_{\xi} z_{\zeta} & y_{\xi} z_{\eta}-y_{\eta} z_{\xi} \\
x_{\zeta} z_{\eta}-x_{\eta} z_{\zeta} & x_{\xi} z_{\zeta}-x_{\zeta} z_{\xi} & x_{\eta} z_{\xi}-x_{\xi} z_{\eta} \\
x_{\eta} y_{\zeta}-x_{\zeta} y_{\eta} & x_{\zeta} y_{\xi}-x_{\xi} y_{\zeta} & x_{\xi} z_{\eta}-x_{\eta} z_{\xi}
\end{array}\right]} \\
J=\left(x_{\xi} y_{\eta} z_{\zeta}+x_{\zeta} y_{\xi} z_{\eta}+x_{\eta} y_{\zeta} z_{\xi}-x_{\xi} y_{\zeta} z_{\eta}-x_{\eta} y_{\xi} z_{\zeta}-x_{\zeta} y_{\eta} z_{\xi}\right)^{-1}
\end{gathered}
$$


The metric terms $\xi_{x}$, etc. are evaluated at grid points $(i, j, k)$ using a conservative, centered scheme. The metric terms at the half points $(i \pm 1 / 2, j, k)$ etc., are computed as simple averages of their values at grid points. Figure 3.1 shows the grid points and the half points.

The equations are nondimensionalized by reference quantities. Here the inlet total density $\rho_{0 \text { ref }}$ and the total sonic velocity $c_{0 \text { ref }}$ were used as reference density and velocity. The length scale is nondimensionalized by one foot (or meter) depending on the units in which the grid is supplied. The Reynolds number Re and Prandtl number Pr are defined in terms of these reference quantities. Quantities such as the specific heats $C_{p}$ and $C_{v}$ and Prandtl number are assumed to be constant in these equations. It is also assumed that the effective viscosity for turbulent flows may be written as

$$
\mu_{\text {eff }}=\mu_{\text {lam }}+\mu_{\text {turb }}
$$

where the laminar viscosity is calculated using a power law function of temperature:

$$
\frac{\mu_{\text {lam }}}{\mu_{\text {ref }}}=\left(\frac{T}{T_{r e f}}\right)^{n}
$$

with $n=2 / 3$ for air.

\subsection{Initial Conditions}

The following initial values were used in the calculation: 


$$
\begin{aligned}
& \rho=\rho_{\infty} \\
& u=0 \\
& v=0 \\
& w=0 \\
& p=p_{\infty} \\
& T=T_{\infty}
\end{aligned}
$$

\subsection{Boundary Conditions}

Boundary conditions for turbomachinery are best expressed in cylindrical

coordinates. But since the boundary conditions in the code are formulated in Cartesian coordinates, coordinate systems are transformed as needed using Equation 3.22. Figure 3.2 shows the transformed coordinate system.

$$
\begin{aligned}
& v=\left(v_{r} y+v_{\theta} z\right) / r \\
& w=\left(v_{r} z-v_{\theta} y\right) / r \\
& v_{r}=(v y+w z) / r \\
& v_{\theta}=(v z-w y) / r \\
& r=\sqrt{y^{2}+z^{2}}
\end{aligned}
$$

\subsubsection{Inflow Boundary Conditions}

At the inflow boundary the total temperature, total pressure, whirl, and meridional flow angle are specified, and the upstream-running Riemann invariant is extrapolated from the interior. In this present study, $v_{\theta}=0$ because no swirl was present at the inlet.

The axial component $u$ and the radial $v_{r}$ are related by the user-specified inflow angle. In supersonic inlets, all flow variables are specified at the inlet. In subsonic flows, the inlet 
conditions are updated every iteration by extrapolating the upstream-running Riemann invariant $\mathrm{R}^{-}$based on the total absolute velocity $Q=\sqrt{u^{2}+v^{2}+w^{2}}$ from the interior to the inlet.

$$
R^{-}=Q-\frac{2 c}{\gamma-1}
$$

The total velocity is found from $\mathrm{T}_{0}$ and $\mathrm{R}^{-}$using

$$
Q=\frac{(\gamma-1) R^{-}+\sqrt{2(1-\gamma)\left(R^{-}\right)^{2}+4(\gamma+1) C_{p} T_{0}}}{\gamma+1}
$$

\subsubsection{Outflow Boundary Conditions}

For subsonic outflow, the exit static pressure is specified and $\rho, \rho u, \rho v$, and $\rho w$ are extrapolated. The local static pressure is found by integrating the axisymmetric radial equilibrium equation:

$$
\frac{\partial p}{\partial r}=\frac{\rho v_{\theta}^{2}}{r}=\frac{\rho}{r^{3}}(v z-w y)^{2}
$$

\subsubsection{Surface Boundary Conditions}

On the surface of the blade and the rotating part of the hub, $U^{\prime}=V^{\prime}=W^{\prime}=0$. Pressure on the blade surface is found from the normal momentum equation. On the hub $(\zeta=1)$ and on the casing $\left(\zeta=\zeta_{\max }\right)$, 


$$
\left(\zeta_{x} \xi_{x}+\zeta_{y} \xi_{y}+\zeta_{z} \xi_{z}\right) \partial_{\xi} p+\left(\zeta_{x} \eta_{x}+\zeta_{y} \eta_{y}+\zeta_{z} \eta_{z}\right) \partial_{\eta} p+\left(\zeta_{x}^{2}+\zeta_{y}^{2}+\zeta_{z}^{2}\right) \partial_{\zeta} p=-\rho\left[\Omega\left(\zeta_{y} w-\zeta_{z} v\right)\right]
$$

On the blade surface $\eta=1$ and the normal momentum equation can be found by replacing $\zeta$ with $\eta$ everywhere in equation (3.26). Temperatures on the blade surface can be obtained from an adiabatic wall condition implemented similarly to equation (3.26) with $p$ replaced by $T$ and a right-hand side of zero.

\subsubsection{Rotor-Stator Interface Boundary Conditions}

The general form of the non-reflecting one-dimensional unsteady boundary conditions developed by Giles $^{96}$ was used here. This method was subsequently implemented by $\mathrm{Chima}^{32}$ in SWIFT code. The node-centered finite difference scheme is used wherein the computational grids overlap by one cell at the interface between two blade rows. A schematic of the overlapping grid, shown in figure 3.3, has been displaced vertically for clarity. After the interior solution on a grid has been updated, the flowfield at the interior boundary is integrated circumferentially at each spanwise location to obtain mass weighted properties such as density, temperature, pressure and enthalpy. This mass

averaged flow vector $\vec{q}$ is stored for use in the boundary conditions on the neighboring grid. The Mach number on the neighboring grid is checked in order to determine whether the flow is supersonic, and the appropriate characteristic boundary conditions are applied. The boundary conditions use the following characteristic variables: 


$$
\left[\begin{array}{l}
C_{1} \\
C_{2} \\
C_{3} \\
C_{4} \\
C_{5}
\end{array}\right]=\left[\begin{array}{ccccc}
-\overline{c^{2}} & 0 & 0 & 0 & 1 \\
0 & \bar{\rho} \bar{c} & 0 & 0 & 1 \\
0 & 0 & \bar{\rho} \bar{c} & 0 & 0 \\
0 & 0 & 0 & \bar{\rho} \bar{c} & 0 \\
0 & -\bar{\rho} \bar{c} & 0 & 0 & 1
\end{array}\right]\left[\begin{array}{c}
\rho-\bar{\rho} \\
v_{x}-\overline{v_{x}} \\
v_{\theta}-\overline{v_{\theta}} \\
v_{r}-\overline{v_{r}} \\
p-\bar{p}
\end{array}\right]
$$

The equation above can be inverted to give:

$$
\left[\begin{array}{c}
\rho-\bar{\rho} \\
v_{x}-\overline{v_{x}} \\
v_{\theta}-\overline{v_{\theta}} \\
v_{r}-\bar{v}_{r} \\
p-\bar{p}
\end{array}\right]=\left[\begin{array}{ccccc}
-1 / \overline{c^{2}} & 1 /\left(2 \overline{c^{2}}\right) & 0 & 0 & 1 /\left(2 \overline{c^{2}}\right) \\
0 & 1 /(2 \bar{\rho} \bar{c}) & 0 & 0 & -1 /(2 \bar{\rho} \bar{c}) \\
0 & 0 & 1 /(\bar{\rho} \bar{c}) & 0 & 0 \\
0 & 0 & 0 & 1 /(\bar{\rho} \bar{c}) & 0 \\
0 & 1 / 2 & 0 & 0 & 1 / 2
\end{array}\right]\left[\begin{array}{l}
C_{1} \\
C_{2} \\
C_{3} \\
C_{4} \\
C_{5}
\end{array}\right]
$$

In equations (3.27) and (3.28) $\mathrm{C}_{\mathrm{i}}$ represent the characteristic variables that correspond to an entropy wave, a downstream-running pressure wave, two vorticity waves, and an upstream-running pressure wave. The other variables in equations (3.27) and (3.28) are: $\rho$ is the density, $p$ is the pressure, $c$ is the speed of sound, and $v_{x}, v_{\theta}$ and $v_{r}$ are velocity components. The bars on some of the quantities refer to average conditions, the coefficient matrices are evaluated at these average conditions. The mixed out average can be applied in a generalized cylindrical coordinate system by equating the 
integrated fluxes to fluxes that are constructed from the average properties. If the $\eta$ coordinate is assumed to coincide with the $\theta$ direction, then

$$
\left[\begin{array}{c}
I_{1} \\
I_{2} \\
I_{3} \\
I_{4} \\
I_{5}
\end{array}\right]=\int J^{-1}\left[\begin{array}{c}
\rho U \\
\rho v_{x} U+\varepsilon_{x} p \\
\rho v_{\theta} U+\left(\varepsilon_{\theta} / r\right) p \\
\rho v_{r} U+\varepsilon_{r} p \\
e+p U
\end{array}\right] d \eta=\left[\begin{array}{c}
\bar{\rho} \bar{U} \\
\bar{\rho} \overline{v_{x}} \bar{U}+\bar{\varepsilon}_{x} \bar{p} \\
\bar{\rho} \overline{v_{x}} \bar{U}+\left(\overline{\varepsilon_{\theta} / r}\right) \bar{p} \\
\bar{\rho} \overline{v_{r}} \bar{U}+\bar{\varepsilon}_{r} \bar{p} \\
(\bar{e}-\bar{p}) \bar{U}
\end{array}\right]
$$

When equation (3.29) is solved, it gives a quadratic equation for $\bar{p}$, the solution for which is:

$$
\begin{aligned}
& \bar{p}=\frac{1}{\gamma+1}\left[a / b \pm \sqrt{(a / b)^{2}+\left(\gamma^{2}-1\right)\left(c-2 I_{1} I_{5}\right) / b}\right] \\
& a=\bar{\varepsilon}_{x} I_{2}+\left(\overline{\varepsilon_{\theta} / r}\right) I_{3}+\bar{\varepsilon}_{r} I_{4} \\
& b=\bar{\varepsilon}_{x}^{2}+\left(\overline{\varepsilon_{\theta} / r}\right)^{2}+\bar{\varepsilon}_{r}^{2} \\
& c=I_{2}^{2}+I_{3}^{2}+I_{4}^{2}
\end{aligned}
$$

For axially subsonic flow the positive root is used. The other average properties follow immediately from 


$$
\begin{aligned}
& \bar{v}_{x}=\left(I_{2}-\bar{\varepsilon}_{x} \bar{p}\right) / I_{1} \\
& \bar{v}_{\theta}=\left(I_{3}-\left(\overline{\varepsilon_{\theta} / r}\right) \bar{p}\right) / I_{1} \\
& \bar{v}_{r}=\left(I_{4}-\bar{\varepsilon}_{r} \bar{p}\right) / I_{1} \\
& \bar{\rho}=I_{1} / \bar{U}
\end{aligned}
$$

Other types of rotor-stator interface boundary conditions have been investigated by Zaki and Iyengar ${ }^{34}$, but it was found that the presently used characteristics boundary conditions ensure the information propagates correctly between blade rows.

\subsection{Time Marching Algorithm}

The governing equations as given in equation (3.1) are discretized using a nodecentered finite difference scheme. Second-order central differences are utilized throughout. The multistage Runge-Kutta scheme as developed by Jameson et $\mathrm{al}^{97}$ is used to advance the flow equations in time from an initial guess to a steady state. Equation (3.1) can be rewritten as follows:

$$
\partial_{t} q=-J\left[R_{I}-\left(R_{V}+D\right)\right]
$$

In equation (3.32) $R_{I}$ is the inviscid residual including the source term, $R_{V}$ is the viscous residual, and $\mathrm{D}_{\mathrm{a}}$ is an artificial dissipation term. The multistage Runge-Kutta algorithm for equation (3.32) can be written in the following manner: 


$$
\begin{aligned}
& q_{0}=q_{n} \\
& q_{1}=q_{0}-\alpha_{1} J \Delta t\left[R_{I} q_{0}-\left(R_{V}+D_{a}\right) q_{0}\right] \\
& \vdots \\
& \vdots \\
& q_{k}=q_{0}-\alpha_{k} J \Delta t\left[R_{I} q_{k-1}-\left(R_{V}+D_{a}\right) q_{0}\right] \\
& q_{n+1}=q_{k}
\end{aligned}
$$

In this study a standard four-stage scheme is used, with $\alpha_{i}=1 / 4,1 / 3,1 / 2,1$. For efficiency, the viscous terms and the artificial dissipation terms are computed only at the first stage. The spatially-varying time step is calculated as the harmonic mean of inviscid and viscous limits in each of the grid directions. Artificial dissipation consisting of blended second and fourth differences is added in order to prevent the point decoupling and also to enhance the stability. The explicit four-stage Runge-Kutta scheme has a Courant stability limit of about 5.5. For a multiblock grid such as the one used in this study, the time step is recalculated every iteration. Implicit residual smoothing after every Runge-Kutta stage is used to increase the maximum Courant number by a factor of two to three, thereby increasing the convergence rate as well. Eigenvalue ${ }^{98-99}$ scaling for both the artificial dissipation and implicit smoothing is used to greatly increase the robustness of the numerical scheme.

\subsection{Turbulence Model}

SWIFT has several turbulence model options. These include the Baldwin-Lomax, Cebeci-Smith, k- $\omega$ baseline and k- $\omega$ SST. Effects of these turbulence models have been investigated and results will be presented subsequently in the validation studies. 
As a part of this study, the k- $\omega$ SST turbulence model is used to model the effects of turbulent mixing in the compression system. The k- $\omega$ SST turbulence model is a high fidelity model and it most accurately captures the complex flow structure in an axial compressor configuration. Wilcox's baseline k- $\omega$ is described in Reference [100], and the implementation of the model in SWIFT is described in Reference [101]. The original k- $\omega$ as proposed by Wilcox has a basic formulation for fully turbulent flows that satisfies the law of the wall without much knowledge of wall distance or a need for complicated nearwall damping terms. The shear stress transport (SST) model was developed by Menter ${ }^{102}$. SST model is based on the assumption that the shear stress in a boundary layer is proportional to $k$.

\subsection{Grid Generation Technique}

A three-dimensional grid code for turbomachinery- TCGRID developed by Chima $^{103}$ at NASA Glenn Research Center is utilized in this study. This code is capable of generating single block grids as well as multi-block grids. The single block grids can be of either the H-type or C-type, whereas the multi-block grids must be a C-type grid around the blade. Blade-to-blade grids are generated using an efficient elliptic solver which gives control over spacing and angles. The following technique is used for grid generation:

1. At first a coarse, equally spaced meridional grid is generated between the specified hub and tip geometries. 
2. The blade coordinates are obtained at the meridional grid points by an interpolation of the input blade geometry.

3. Next, the two-dimensional blade-to-blade grids are generated along the meridional grid lines. This two-dimensional grid is generated in the $(m, \bar{r} \theta)$ coordinates using a version of the GRAPE code developed by Steger and Sorenson ${ }^{104}$. The $m$ direction refers to the arc length along the meridional surface, and $\bar{r}$ is some mean radius.

4. The $(m, \bar{r} \theta)$ coordinates are transformed back to $(z, r, \theta)$ coordinates.

5. Now that the two-dimensional grids are in $(z, r, \theta)$ coordinates, they are reclustered spanwise using a hyperbolic tangent stretching function in order to make a full three-dimensional grid.

6. Finally, the three-dimension grid is transformed from the $(z, r, \theta)$ coordinates to the $(x, y, z)$ coordinates and stored in a standard format.

\subsection{Design Tools}

This study uses a number of tools to suffice the design process. A method to parametrically design the axial compressor blades is presented in Section 3.7.1. When designing the blades, a number of design variables many be involved. The Design of Experiments technique used to sift through these design variables is discussed briefly in Section 3.7.2. An optimization method is needed to obtain the optimum settings for the 
design variables considered. Such a technique called the Response Surface Method is introduced in Section 3.7.3.

\subsubsection{Parametric Blade Design}

In an effort to parametrically model the compressor blades in this study, a tool

originally developed by Wood $^{105}$ called CCGEOM, is used. The tool can be used to facilitate the rapid generation of the flow passage and blading for turbomachinery components.

The tool uses a piece-wise smooth cubic spline interpolation method to obtain the geometry. First, the hub and tip geometries are supplied by the user as inputs by specifying the cylindrical co-ordinates (radial and axial) along each surface. Blade mean camber line angle and thickness at various chord-wise points and at different spanwise locations are specified as user-input. Based on the hub and tip geometry along with the supplied blade camber line angles and thicknesses, the blade surfaces are interpolated using a piece-wise cubic spline interpolation. In this study blade camber line angles and thicknesses at three chord-wise (leading edge, mid-chord and trailing edge) points and three different (hub, mid-span and tip) spanwise locations were specified for convenience. The present methodology is not restricted to specification of the blade geometry at these three locations. For more complex geometries, blade geometry may be specified at more locations. Figure 3.2 summarizes the variables used for the parametric design of the blade. 
The blade is stacked at ten spanwise locations; all at $33 \%$ of the chord location by specifying the leaning angle at each location. This is done after the spanwise surfaces are generated in the R-Z plane. The blade normal thickness from hub to tip is obtained by specifying a moderate parabolic shape function that has zero slope of thickness at the tip. This ensures that the thickness is smoothly distributed hence removing the need for maximum amount of metal for a given hub thickness to that will help relieve high hub stresses.

\subsubsection{Design of Experiments}

The above parametric model gives rise to a number of parameters or "design variables" that may be generated to produce a family of blade surfaces. An independent change (say two possible changes) to each of these $\mathrm{N}$ values will give rise to $2^{\mathrm{N}}$ combinations, a very large design space. Design of Experiments (DoE) is a systematic way to plan, conduct and analyze a series of tests, where the input variables are changed systematically to extract intelligent information. This method uses a statistical approach that predicts the influences of variables along with their interactions on the responses, without the need for a full factorial analysis. By using DoE, the needed information can be extracted with less time and money. Several methods have been developed to carry out the design of experiments, but the main purpose of each method is the same, which is to reduce the number of cases required to run whilst extracting more information from them. 


\subsubsection{Response Surface Methodology}

For this work, the use of an all-encompassing model of the physical environment is required in order to explore the design space. The exploration of a complex design space requires the use of a Response Surface Methodology (RSM). Lawson and Madrigal $^{106}$ pointed out RSM models were as efficient in optimizing the relationship between input design factors and desired responses as compared to other optimizing techniques such as Monte Carlo simulation, nonlinear optimization and dynamic programming. In this study, a commercial software- JMP $\odot$ is used to perform the response surface optimization.

RSM acts as a means to find the optimal settings of input factors or design variables that maximize, minimize or target measured responses or outcome variables. It utilizes Response Surface Equations (RSE's) that take the form of a polynomial approximation of the relationships across given ranges for the input variables. A mathematical representation of the second-degree model of the equation is given in (3.34).

$$
R=b_{0}+\sum b_{i} x_{i}+\sum b_{i i} x_{i}^{2}+\sum \sum b_{i j} x_{i} x_{j}
$$

Here $b_{0}$ is the intercept and $b_{n}$ the respective regression coefficients of the subsequent terms. Variables $x_{i}, x_{j}$ represents design inputs that influence the response.

In order to obtain the regression coefficients, the responses from all the DOE cases are collected and the RSE is generated using the Least Square Method (LSM). For a 
given set of points, the LSM obtains the best-fit equation by minimizing the sum of squares of the vertical deviation between the equation and the point. The sum of the squares of the vertical deviation is calculated according by (3.35), the coefficients for the best-fitting RSE are found by differentiating $\mathrm{R}^{2}$ and setting it to zero, as shown in (3.36)

$$
\begin{gathered}
R^{2}=\sum\left[y_{i}-f\left(x_{i}, a_{1}, a_{2}, \ldots ., a_{n}\right)\right]^{2} \\
\frac{\partial\left(R^{2}\right)}{\partial a_{i}}=0
\end{gathered}
$$

Upon obtaining the response surface it is examined to determine if the optimum operating conditions (values of input variables) reaches the maximum, minimum, ridge, or saddle point region for the response variables. Eigenvalue and eigenvectors help identify the shape and curvature orientation of the response surface. If the optimum region is not reached, exploration continues in the direction towards the maximum (if there are negative eigenvalue signs associated with the stationary point), towards the minimum (if there are positive eigenvalue signs associated with the stationary point) or towards the saddle point (if there are different eigenvalue signs). A zero eigenvalue indicates a flat fitted surface.

Once the response surface is generated, the optimization procedure is done using the desirability function ${ }^{107}$. The desirability function is a transformation where the response function is rescaled to a 0 to 1 scale. Using a scaled response has the advantage that the overall result does not depend on the scales of the original responses. Here a response of 0 represents a completely undesirable response and 1 represents the most 
desirable response. For a equally weighted multi-response, each of the transformed response $\mathrm{d}_{\mathrm{i}}$, are combined using the geometric mean to create the overall desirability function (D) as given in (3.37),

$$
D=\sqrt[n]{\left(d_{1} \times d_{2} \times d_{3} \times \ldots d_{n}\right)}
$$

where $d_{1} . . d_{n}$ transformed responses for $R_{1} . . R_{n}$.

By using the product of the desirability functions it insures that if any single desirability is 0 (undesirable), then the overall desirability is 0 . Hence, the simultaneous optimization of several individual responses has been reduced to optimizing a single response: the overall desirability function, D. Equation (3.37) may be rewritten for incorporating differential weighting and takes the form as shown in (3.38).

$$
D=A_{1} d_{1}+A_{2} d_{2}+A_{3} d_{3}+\ldots . A_{n} d_{n}
$$

Here $A_{1 \ldots . n}$ are the weighting assigned to each of the responses. For the first part of the study the total pressure ratio $\left(\mathrm{A}_{1}\right)$ and adiabatic efficiency $\left(\mathrm{A}_{2}\right)$ are assigned en equal weighting of 1 . In the second part of the study an aeroacoustic- rotor wake influence coefficient $\left(\mathrm{A}_{3}\right)$ is also introduced and it is equally weighted as total pressure ratio and adiabatic efficiency.

In such a case when more than one response has to be optimized, a multi objective problem, a complete different situation arises. The definition of optimality (global) in the 
single response case must be dropped; it is a very unlikely situation that a setting of the independent variables is found which holds the desired optimum for all the responses at once. In practice the experimenter/designer has to search for an acceptable compromise between the different objectives. While in the single objective case one can speak about 'the optimum', in the multi objective case there is not one particular optimum. Depending on the relative preferences of the experimenter/designer towards the individual objectives, the definition of the acceptable compromise will also have a different meaning and a different solution will be found. Through the weights of the desirability function relative preferences on the designer can be changed. Much work remains to be done on the optimum weights for these responses.

\subsection{Total Pressure Ratio and Adiabatic Efficiency Calculation}

In a compression system the overall pressure ratio increases. The total pressure ratio is a measure of the increase in the pressure of the compression system. Total pressure ratio (TPR) of a compression system is defined as the ratio between the total pressure exiting the compressor to the total pressure entering the compressor. If the

pressure exiting the compressor is $P_{02}$ and the pressure entering the compressor is $P_{01}$ then the TPR is defined as:

$$
T P R=\frac{P_{02}}{P_{01}}
$$


The adiabatic efficiency of a compressor is the ratio of the ideal input work needed to raise the total pressure of a working fluid from a pressure value $P_{01}$ to a new value $P_{02}$, to the actual work needed on the fluid. The adiabatic efficiency $\left(\eta_{a d}\right)$ of the compression system can be found by using:

$$
\eta_{a d}=\frac{W_{S}}{W_{A}}
$$

where $W_{S}$ and $W_{A}$ are the isentropic and actual work done on the flow, respectively. These can be found as follows:

$$
\begin{gathered}
W_{S}=\dot{m} c_{p}\left(\bar{T}_{02 \mid s}-\bar{T}_{01}\right) \\
W_{A}=\dot{m} c_{p}\left(\bar{T}_{02}-\bar{T}_{01}\right)
\end{gathered}
$$

where $\bar{T}_{01}$ and $\bar{T}_{02}$ are the total temperature are the original total temperature and the new total temperatures respectively.

By applying isentropic relationship for points 1 and 2 as follows:

$$
\left.\frac{\bar{T}_{02}}{\bar{T}_{01}}\right|_{s}=\left(\frac{\bar{p}_{02}}{\bar{p}_{01}}\right)^{\frac{\gamma-1}{\gamma}}
$$

the bar quantities $\bar{p}, T$ are obtained by the mixed-out averaging technique previously discussed in section 3.3.4. By substituting equations 3.41 and 3.42 into equation 3.40, we get : 


$$
\eta_{a d}=\frac{\left(\frac{\bar{p}_{02}}{\bar{p}_{01}}\right)^{\frac{\gamma-1}{\gamma}}-1}{\frac{\bar{T}_{02}}{\bar{T}_{01}}-1}
$$

\subsection{Aeroacoustic Analysis Technique}

In order to gain accurate noise prediction via $\mathrm{CFD}$, it is important to ensure the boundary conditions used impose non-reflecting boundary conditions. The boundary conditions as discussed in Section 3.3, ensures that non-reflecting properties are imposed at the boundaries. The acoustic method adopted in this study computes the rotorwake/stator interactions through coupled computation. Rotor wake profiles are established with three dimensional computations.

A Rotor Wake Influence Coefficient (RWIC) is defined to estimate the acoustic sound level generated by the rotor-wake/stator interactions at the rotor/stator exit. Figure 3.3 shows the axial and circumferential location where the RWIC is calculated. Here RWIC is defined as:

$$
R W I C=10 \log _{10}\left(P_{A M P} / P_{R E F}\right)
$$

where $\mathrm{P}_{\mathrm{REF}}$ is the reference power equal to $20 \mu \mathrm{Pa}$ and $\mathrm{P}_{\mathrm{AMP}}$ is the root mean square fluctuating pressure amplitude at the rotor/stator interface defined as: 


$$
P_{A M P}^{\prime}=\sqrt{\left(\frac{P_{1}^{\prime 2}+P_{2}^{\prime 2}+P_{3}^{\prime 2}+\cdots+P_{n}^{\prime 2}}{n}\right)}
$$

where $P_{1}^{\prime}, P_{2}^{\prime} \ldots . P_{n}^{\prime}$ are the fluctuating pressures on each blade-to-blade mesh point at mid-span. The fluctuations are measured based on a circumferentially averaged pressure quantity at mid-span. Pressures at each blade-to-blade mesh point at mid span are subtracted from the circumferentially averaged pressure to give the fluctuating pressure profile. Upon obtaining the RWIC at each point, the RWIC value will be used in the optimization loop as an objective function. This RWIC value provides a way to measure the acoustic disturbance generated by the rotor wake onto the stator.

Smith $^{108}$ et. al. have reported that compressor noise is more predominant in the upstream direction. The noise sources contributing to compressor noise can originate at different locations in the compression system- on the blade, in the tip gap, rotor-stator interface etc. Typically noise generated due to rotor/stator noise interaction noise is considered to be important when carrying out a compressor stage design. Hence the use of RWIC at rotor-stator interface as an objective function is justified. In the postprocessing analysis presented later in this work, the effect of the upstream acoustic noise from rotor-stator interactions will also be investigated. 


\subsection{Structural Analysis}

A commercial computational structural dynamics (CSD) solver- ANSYS is used to perform static structural analysis. ANSYS is coupled with the CFD solver- SWIFT in an open looped fashion in order to account for the blade deflections under normal operating conditions. The CFD grid for the blade is converted into an ANSYS input using a script glyph file. Pressure data on the blade surface are obtained from the CFD analysis. The extracted blade pressure is then interpolated from the CFD grid to the ANSYS grid. After which static structural analysis is performed on the blade to find the maximal von Mises stress on the blade and the deflections.

Commercial software ANSYS solves the following structural equilibrium equation:

$$
K d=F
$$

where $K$ is the stiffness matrix, $d$ is the displacement vector, and $F$ is the force vector, which includes the aerodynamic pressure force and centrifugal force.

\subsection{Selection of Design Variables}

The selection of design variables in this study were done in two stages. First, in the preliminary application of the methodology the design variables were chosen by engineering-based intuition. Then in the extended methodology the design variables are

ranked based on its variability with the objective functions. The method of selecting the 
important design variables for the extended methodology will be discussed in this section.

The DoE method used to narrow down the important variables has been adapted from a method initially proposed by Cotter $^{109}$. Cotter initially suggested $2 n+2$ runs: one run with all variables set at their lowest setting is done. This is followed by $\mathrm{n}$ runs with each successive variables set at its highest setting while all other variables fixed at the lowest setting. Next another $\mathrm{n}$ runs with each successive variable set at its lowest setting with all the variables fixed at the highest setting are done, followed by one run with all variables set at their highest settings. Similar analysis has also been used by Nixon ${ }^{110}$. In this present study an extra run in added- with all variables set to its zero value, hence resulting in $2 n+3$ runs. Here the zero value represents the non perturbed value of the design variable, -1 represents the lowest setting of the design variable and +1 represents the highest settings of the design variable. Table 3.1 shows the design of experiments constructed for 18 variables considered in this case. In table 3.1, the case represent all variables set at their lowest setting, followed by 19 cases with each successive variable set at its highest setting while all other variables fixed at the lowest setting. A further set of 19 runs with each successive variable set at its lowest setting with all the variables fixed at the highest setting are made, followed by one run with all variables set at their highest settings, and one run with all variables at zero values.

The experiment, which in this case is a CFD based simulation, is conducted for each case. Responses are recorded for these runs, as this information is crucial to the later step for screening. In most cases, designers select variable ranges so that they represent 
feasible designs, and as a result minimize the number of failures in the experiment sets. In a design environment, a failed experiment set corresponds to a set that is outside the design space. More often than not an extreme perturbation of the design variable can be lead to exaggerated changes and result in a failed experiment. By trying to minimize the number of failures in the experiment sets there is the risk of overly limiting variable ranges. As a general rule, small changes to the shape of the compressor blade can have significant effects on the compressor performance and at times can be the reason for worse than anticipated performance. Hence selecting the range in this case is a challenging task. Here the upper and lower limits for each variable are obtained on an individual basis by identifying the failure values. Doing so allows a much more precise location of the constraints in order to avoid unnecessarily limiting the design space.

After the design space is defined for all the design variables, a method to screen the more important design variables is needed. Thus, the goal of the screening step is to determine which variables significantly affect the responses. A commonly used statistical technique called ANOVA (Analysis of Variance) provides a very convenient way to compare the means of several variables. ANOVA is a collection of statistical models, and their associated procedures, in which the observed variance is partitioned into components due to different explanatory variables, usually called factors in design of experiments. Reference [111]-[112] give a detailed description of the analysis of variance technique.

With variance as measure of variability, the ANOVA analysis provides information regarding how each design parameter varies with each response. A Pareto 
plot $^{113}$ is used to show the individual influence of each design variable on the response. As an example a typical Pareto curve showing the growth of four commodities- wheat, soy, oats and rye is shown in figure 3.4. The Pareto plot is a type of plot used in quality control applications that combines a bar chart displaying percentages of categories in the data with a line graph showing cumulative percentages of the categories. Clearly, wheat, soy and oats are in abundance as compared to rye. Although the response here is just commodity growth, this Pareto plot shows that it is favorable to grow wheat, soy and oats rather than rye.

In a multi-objective design there are multiple responses (total pressure ratio, efficiencies, RWIC), a Pareto plot can be generated for each response and the variables contributing to all the responses can be identified. The selection of important contributing variables is based on the Pareto Principle. Pareto postulated that for many phenomena, $80 \%$ of the consequences stem from $20 \%$ of the causes. When applied to this study, the Pareto idea states that $80 \%$ of the variability in a particular response is due to $20 \%$ of the variables involved.

Sometimes each of the variables can contribute the same amount to the responses. In fact, their effects could be indistinguishable. This is where engineering knowledge and experience as to which variable to choose becomes crucial. Since this is a screening test that considers linear effects, some variables may show up as significant, when in fact they are not. 


\begin{tabular}{|c|c|c|c|c|c|c|c|c|c|c|c|c|c|c|c|c|c|c|}
\hline & $A$ & $B$ & C & $\mathrm{D}$ & $E$ & $\mathrm{~F}$ & $G$ & $\mathrm{H}$ & 1 & $\mathrm{~J}$ & $\mathrm{~K}$ & $\mathrm{~L}$ & $M$ & $\mathrm{~N}$ & $\mathrm{O}$ & $P$ & $Q$ & $\mathrm{R}$ \\
\hline 1 & -1 & -1 & -1 & -1 & -1 & -1 & -1 & -1 & -1 & -1 & -1 & -1 & -1 & -1 & -1 & -1 & -1 & -1 \\
\hline 2 & 1 & -1 & -1 & -1 & -1 & -1 & -1 & -1 & -1 & -1 & -1 & -1 & -1 & -1 & -1 & -1 & -1 & -1 \\
\hline 3 & -1 & 1 & -1 & -1 & -1 & -1 & -1 & -1 & -1 & -1 & -1 & -1 & -1 & -1 & -1 & -1 & -1 & -1 \\
\hline 4 & -1 & -1 & 1 & -1 & -1 & -1 & -1 & -1 & -1 & -1 & -1 & -1 & -1 & -1 & -1 & -1 & -1 & -1 \\
\hline 5 & -1 & -1 & -1 & 1 & -1 & -1 & -1 & -1 & -1 & -1 & -1 & -1 & -1 & -1 & -1 & -1 & -1 & -1 \\
\hline 6 & -1 & -1 & -1 & -1 & 1 & -1 & -1 & -1 & -1 & -1 & -1 & -1 & -1 & -1 & -1 & -1 & -1 & -1 \\
\hline 7 & -1 & -1 & -1 & -1 & -1 & 1 & -1 & -1 & -1 & -1 & -1 & -1 & -1 & -1 & -1 & -1 & -1 & -1 \\
\hline 8 & -1 & -1 & -1 & -1 & -1 & -1 & 1 & -1 & -1 & -1 & -1 & -1 & -1 & -1 & -1 & -1 & -1 & -1 \\
\hline 9 & -1 & -1 & -1 & -1 & -1 & -1 & -1 & 1 & -1 & -1 & -1 & -1 & -1 & -1 & -1 & -1 & -1 & -1 \\
\hline 10 & -1 & -1 & -1 & -1 & -1 & -1 & -1 & -1 & 1 & -1 & -1 & -1 & -1 & -1 & -1 & -1 & -1 & -1 \\
\hline 11 & -1 & -1 & -1 & -1 & -1 & -1 & -1 & -1 & -1 & 1 & -1 & -1 & -1 & -1 & -1 & -1 & -1 & -1 \\
\hline 12 & -1 & -1 & -1 & -1 & -1 & -1 & -1 & -1 & -1 & -1 & 1 & -1 & -1 & -1 & -1 & -1 & -1 & -1 \\
\hline 13 & -1 & -1 & -1 & -1 & -1 & -1 & -1 & -1 & -1 & -1 & -1 & 1 & -1 & -1 & -1 & -1 & -1 & -1 \\
\hline 14 & -1 & -1 & -1 & -1 & -1 & -1 & -1 & -1 & -1 & -1 & -1 & -1 & 1 & -1 & -1 & -1 & -1 & -1 \\
\hline 15 & -1 & -1 & -1 & -1 & -1 & -1 & -1 & -1 & -1 & -1 & -1 & -1 & -1 & 1 & -1 & -1 & -1 & -1 \\
\hline 16 & -1 & -1 & -1 & -1 & -1 & -1 & -1 & -1 & -1 & -1 & -1 & -1 & -1 & -1 & 1 & -1 & -1 & -1 \\
\hline 17 & -1 & -1 & -1 & -1 & -1 & -1 & -1 & -1 & -1 & -1 & -1 & -1 & -1 & -1 & -1 & 1 & -1 & -1 \\
\hline 18 & -1 & -1 & -1 & -1 & -1 & -1 & -1 & -1 & -1 & -1 & -1 & -1 & -1 & -1 & -1 & -1 & 1 & -1 \\
\hline 19 & -1 & -1 & -1 & -1 & -1 & -1 & -1 & -1 & -1 & -1 & -1 & -1 & -1 & -1 & -1 & -1 & -1 & 1 \\
\hline 20 & -1 & 1 & 1 & 1 & 1 & 1 & 1 & 1 & 1 & 1 & 1 & 1 & 1 & 1 & 1 & 1 & 1 & 1 \\
\hline 21 & 1 & -1 & 1 & 1 & 1 & 1 & 1 & 1 & 1 & 1 & 1 & 1 & 1 & 1 & 1 & 1 & 1 & 1 \\
\hline 22 & 1 & 1 & -1 & 1 & 1 & 1 & 1 & 1 & 1 & 1 & 1 & 1 & 1 & 1 & 1 & 1 & 1 & 1 \\
\hline 23 & 1 & 1 & 1 & -1 & 1 & 1 & 1 & 1 & 1 & 1 & 1 & 1 & 1 & 1 & 1 & 1 & 1 & 1 \\
\hline 24 & 1 & 1 & 1 & 1 & -1 & 1 & 1 & 1 & 1 & 1 & 1 & 1 & 1 & 1 & 1 & 1 & 1 & 1 \\
\hline 25 & 1 & 1 & 1 & 1 & 1 & -1 & 1 & 1 & 1 & 1 & 1 & 1 & 1 & 1 & 1 & 1 & 1 & 1 \\
\hline 26 & 1 & 1 & 1 & 1 & 1 & 1 & -1 & 1 & 1 & 1 & 1 & 1 & 1 & 1 & 1 & 1 & 1 & 1 \\
\hline 27 & 1 & 1 & 1 & 1 & 1 & 1 & 1 & -1 & 1 & 1 & 1 & 1 & 1 & 1 & 1 & 1 & 1 & 1 \\
\hline 28 & 1 & 1 & 1 & 1 & 1 & 1 & 1 & 1 & -1 & 1 & 1 & 1 & 1 & 1 & 1 & 1 & 1 & 1 \\
\hline 29 & 1 & 1 & 1 & 1 & 1 & 1 & 1 & 1 & 1 & -1 & 1 & 1 & 1 & 1 & 1 & 1 & 1 & 1 \\
\hline 30 & 1 & 1 & 1 & 1 & 1 & 1 & 1 & 1 & 1 & 1 & -1 & 1 & 1 & 1 & 1 & 1 & 1 & 1 \\
\hline 31 & 1 & 1 & 1 & 1 & 1 & 1 & 1 & 1 & 1 & 1 & 1 & -1 & 1 & 1 & 1 & 1 & 1 & 1 \\
\hline 32 & 1 & 1 & 1 & 1 & 1 & 1 & 1 & 1 & 1 & 1 & 1 & 1 & -1 & 1 & 1 & 1 & 1 & 1 \\
\hline 33 & 1 & 1 & 1 & 1 & 1 & 1 & 1 & 1 & 1 & 1 & 1 & 1 & 1 & -1 & 1 & 1 & 1 & 1 \\
\hline 34 & 1 & 1 & 1 & 1 & 1 & 1 & 1 & 1 & 1 & 1 & 1 & 1 & 1 & 1 & -1 & 1 & 1 & 1 \\
\hline 35 & 1 & 1 & 1 & 1 & 1 & 1 & 1 & 1 & 1 & 1 & 1 & 1 & 1 & 1 & 1 & -1 & 1 & 1 \\
\hline 36 & 1 & 1 & 1 & 1 & 1 & 1 & 1 & 1 & 1 & 1 & 1 & 1 & 1 & 1 & 1 & 1 & -1 & 1 \\
\hline 37 & 1 & 1 & 1 & 1 & 1 & 1 & 1 & 1 & 1 & 1 & 1 & 1 & 1 & 1 & 1 & 1 & 1 & -1 \\
\hline 38 & 1 & 1 & 1 & 1 & 1 & 1 & 1 & 1 & 1 & 1 & 1 & 1 & 1 & 1 & 1 & 1 & 1 & 1 \\
\hline 39 & 0 & 0 & 0 & 0 & 0 & 0 & 0 & 0 & 0 & 0 & 0 & 0 & 0 & 0 & 0 & 0 & 0 & 0 \\
\hline
\end{tabular}

Table 3.1: Design of experiment cases for the 18 design variables investigated. 


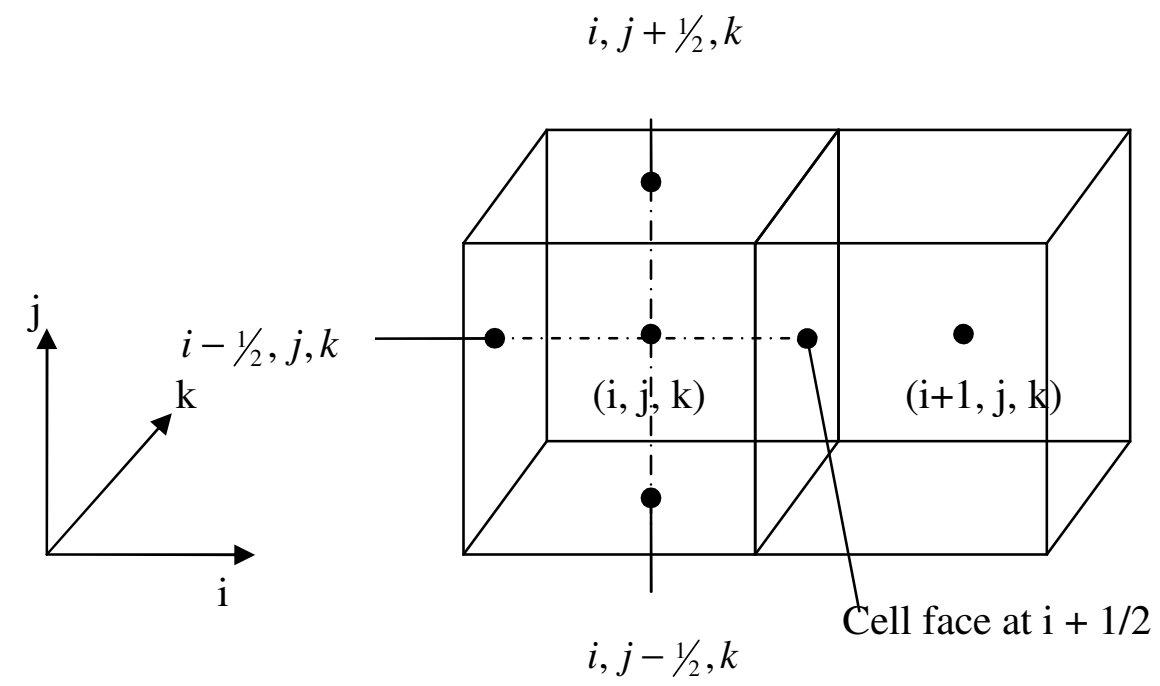

Figure 3.1: Control Volume and cell-vertex grid points.

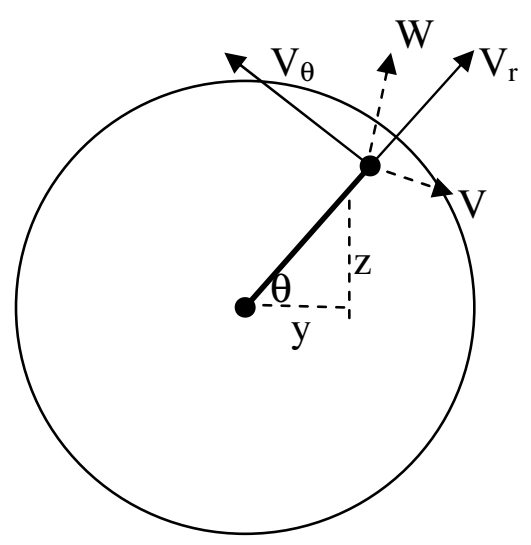

Figure 3.2: Cartesian to Cylindrical coordinates transformation. 


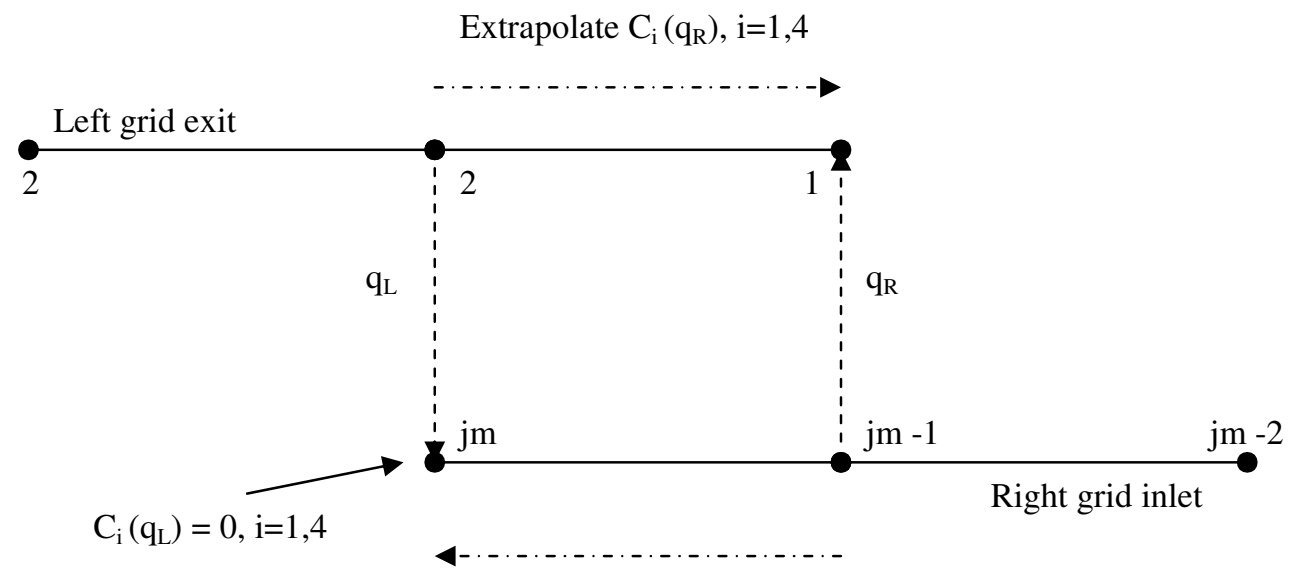

Extrapolate $\mathrm{C}_{5}\left(\mathrm{q}_{\mathrm{L}}\right)$

Figure 3.3: Schematic diagram showing the implementation of characteristic boundary condition at rotor-stator interface.

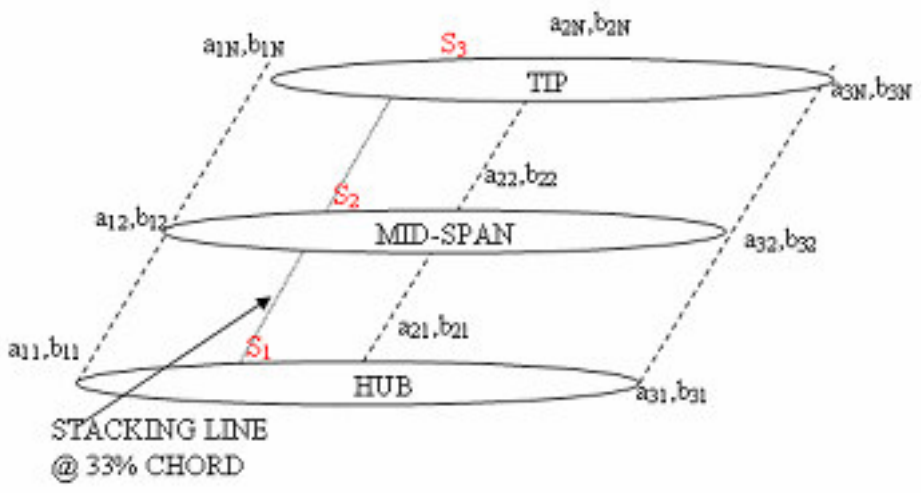

$a_{M N}=$ Blade mean camber line angle at chord location ' $M$ 'and span location ' $N$ '

$b_{M, N}=$ Blade thickness at chord location ' $M$ ' and span location $N$ '

$S_{N}=$ Blade section at span location ' $N$ '

$f\left(S_{1}\right)=f\left(a_{1,1}, b_{1,1}, a_{2,1}, b_{2,1}, a_{3,1}, b_{3,1}\right)$

$f\left(S_{N}\right)=f\left(a_{N, 1}, b_{N, 1}, a_{N, 1}, b_{N, 1}, a_{N, 1}, b_{N, 1}\right)$

$f\left(B L A D E_{x, y, z}\right)=f\left(S_{1}, S_{2}, S_{3} \ldots \ldots S_{N}\right) s$

$f(x)$ - piece-wise smooth cubic spline interpolation

Figure 3.4: Summary of parametric blade design based on design variables- $a_{M, N}, b_{M, N}$ 


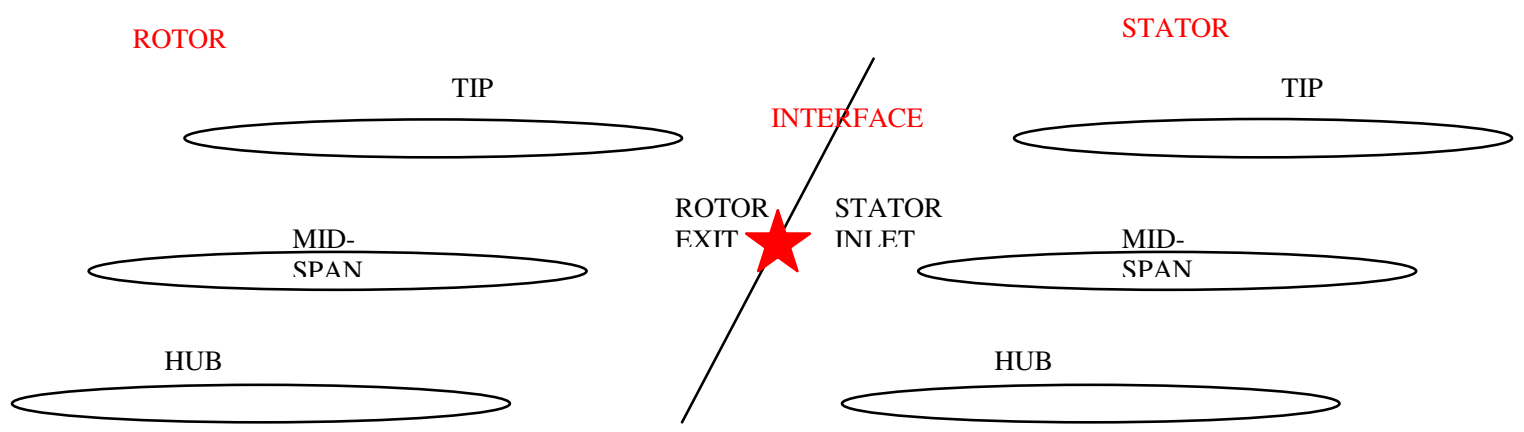

Figure 3.5: Schematic diagram showing the interface location used for the aeroacoustic analysis.

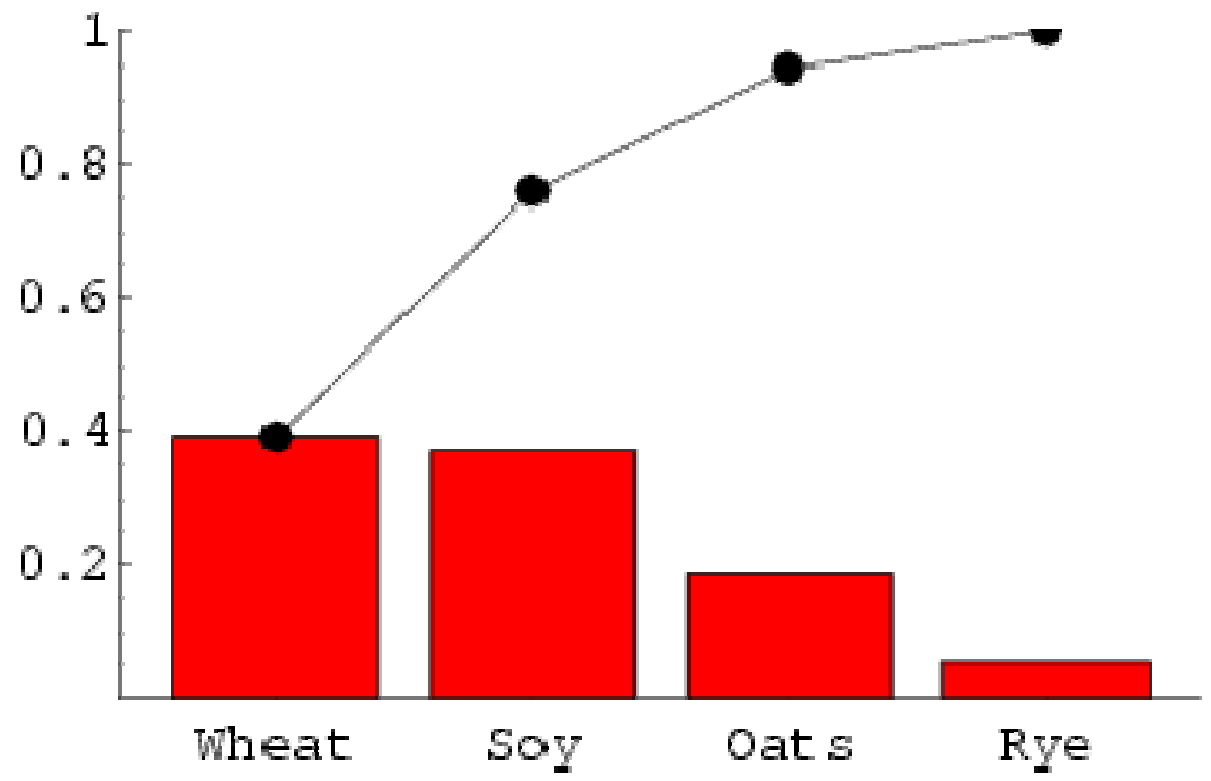

Figure 3.6: An example to illustrate the Pareto Curve.

Ref- http://mathworld.wolfram.com/ParetoPlot.html 


\section{CHAPTER IV}

\section{VALIDATION STUDIES}

This chapter will present the results from validation studies performed on the Navier-Stokes solver SWIFT $^{94}$ using the associated grid generator TCGRID ${ }^{103}$. Descriptions of all the configurations and the computational grids are discussed prior to presentation of the validation results.

The chapter is organized as follows. The validation results for a commonly used NASA Rotor 67 configuration are presented in Section 4.1. In section 4.2, results obtained from the analysis of a Goldman Turbine Vane are presented. Results from analysis performed on the Space Shuttle Main Engine (SSME) single stage turbine are presented in Section 4.3.

\subsection{NASA Transonic Axial Rotor 67}

The first geometry chosen for the validation studies was a transonic high-speed axial fan rotor, called NASA Rotor 67. There is a large body of experimental and computed data available for controlled and baseline configurations. This low aspect ratio rotor $(\sigma=1.56)$ is the first stage of a two stage transonic fan designed and tested NASA Glenn Research Center. Measured performance maps as well as LDV velocity fields are available. The rotor has 22 blades; the design pressure ratio is 1.63 , at a mass flow rate of 
$33.25 \mathrm{~kg} / \mathrm{sec}$. The design rotational speed is $16043 \mathrm{RPM}$, which yields a tip leading edge speed of $429 \mathrm{~m} / \mathrm{sec}$ with an inlet tip relative mach number of 1.38. A detailed description of this configuration along with measured data may be found in Reference [114].

A nominal H-type grid, shown in figure 4.1 is used to model the Rotor 67 configurations. The single-block grid generated using TCGRID, has 151 cells in the streamwise direction, 54 cells in the radial direction and 49 cells in the circumferential direction. Grid sensitivity studies were performed by Chima, it was found that the results are independent of the overall grid dimension. The grid dimensions used for validation of the Rotor 67 configuration are based on engineering intuition and are justifiable. The k- $\omega$ SST turbulence model is used to model the turbulent flow for this calculation. Solutions for every operating point is obtained after approximately 2000 iterations (70 minutes) on a Pentium $42.80 \mathrm{GHz}$ 512MB PC.

The performance map computed for the Rotor 67 geometry was compared with the experimental map obtained by Reid and Moore ${ }^{115}$. In the map, as shown in figure 4.2, the total pressure ratio of the stage is plotted as function of the ratio of the mass flow rate to the choking mass flow rate. It is customary that mass flow is normalized by the mass flow rate at choked flow condition as to avoid distortion between the relative shapes of the curves and for removing the differences in the computed choking mass flow rate as factor in the design process.

The choking mass flow rate for the Rotor 67 configuration was computationally found to be $34.56 \mathrm{~kg} / \mathrm{sec}$; experimental choking mass flow rate is reported as 34.96 $\mathrm{kg} / \mathrm{sec}$. The performance map shows very good agreement with the experimental values, 
although the stall point was not captured accurately. The adiabatic efficiency map is shown on Figure 4.3, again very good agreement with experimental values is observed.

\subsection{Goldman Turbine Vane}

An annular turbine vane that was tested experimentally by Goldman and McLallin ${ }^{116}$ at NASA Glenn Research Center was used as a test for three dimensional turbines. The stator has 36 vanes. The vanes have an axial chord length of $0.03823 \mathrm{~m}$, a span (between the hub and tip) of $0.0381 \mathrm{~m}$, a $0.508 \mathrm{~m}$ tip diameter and a hub to tip radius of 0.85 . It has a design pressure ratio of 0.6705 , inlet Mach number of 0.211 , exit Mach number of 0.655 and Reynolds number based on the axial chord of 173,000.

A C-type computational grid, as shown in Figure 4.4, has 97 streamwise cells, 32 radial cells and 33 cells in the circumferential direction. The grid generated using TCGRID, was rather coarse, but it gave reasonably accurate predictions of vane performance with quick turnaround. The grid spacing gave a $\mathrm{y}^{+}=\mathrm{O}(5)$ over most part of the vane. The solutions were obtained for both the Baldwin-Lomax and the Cebeci-Smith turbulence models. Results were found to be independent of the turbulence model. Here the results reported are for the Cebeci-Smith turbulence model. On a Pentium $42.80 \mathrm{GHz}$ 512MB PC, the solution takes approximately 80 minutes.

The Mach number contour at a typical radial location (mid-span) is shown in

figure 4.5. The contours show very good agreement with the published data ${ }^{117}$. The surface pressure distribution at the same radial location is shown in figure 4.6. It is seen 
that the computed solution agrees very well the measured data over most of the blade surface.

\subsection{Space Shuttle Main Engine (SSME) Single Stage Turbine}

As a final validation, the first stage of the space shuttle main engine was studied. This engine has two turbopumps to pump the fuel and oxidizer from the main tank to the combustion chamber. Each high pressure turbopump uses a two-stage axial flow turbine in order to drive the pump. The high-pressure fuel turbine (HPFT) was experimentally tested by Hudson ${ }^{118}$ et al. at NASA Marshall Space Flight Center in a cold-flow test. Dunn $^{119}$ et al. measured blade surface heat transfer and unsteady pressures at Calspan in a short-duration shock tube. The first stage of this two stage fuel turbine is analyzed for purpose of validating the solver with a full stage configuration. The stage operates at an inlet mach number of 0.132 and has a design pressure ratio of 0.65 .

The computational grid was generated using TCGRID. Grids were generated for each blade separately and were then combined with a utility code such that each grid overlapped its neighbor by one cell. The grid dimensions for the stator, rotor and the rotor tip clearance are summarized below;

- $\quad$ Stator C-Grid: 127 x 37 x $45=211,455$ points.

- $\quad$ Rotor C-Grid: 127 x 33 x $45=188,595$ points.

- $\quad$ Rotor clearance O-Grid: 95 x 13 x $11=13,585$ points. 
A streamwise view of the grid is shown in figure 4.7 along with the rotor tip clearance O-grid. At the walls, a no-slip boundary condition was applied and the pressure gradient was set to zero. A wall/gas temperature ratio of 0.7 was used for this analysis; this was done to simulate the nominal experimental conditions. The Cebeci-Smith Turbulence model are used and the nominal initial grid spacing's in turbulent wall units were $\mathrm{y}^{+}=2.5$ on blades, $\mathrm{y}^{+}=3.5$ on endwalls, and $\mathrm{y}^{+}=7.0$ on the rotor tips. It has been reported $^{94}$ that the Cebeci-Smith turbulence model works well for the turbine configuration but may overpredict losses for transonic compressor. Fully converged solutions took approximately 80 minutes on a Pentium $42.80 \mathrm{GHz} 512 \mathrm{MB}$ PC.

The flow field inside the rotor-stator passages was examined through a study of the Mach number contours obtained and compared to published results ${ }^{29}$. Figure 4.8 compares the contours of absolute Mach number through the turbine at mid-span. It can be observed that the computation shows good agreement with previously published data. Figure 4.9 compares computed and measured static pressures at various locations through the turbine. Again, the results show very good comparison with experimental data and previously published results.

Figures 4.10-4.11 show comparisons between computed and measured surface Stanton numbers at mid-span plotted against unwrapped surface distance for the stator and rotor respectively. Results from the computational analysis show good agreement with measured data both in the case of the rotor and the stator. The computations were run assuming that the flow was fully-turbulent. 


\subsection{Baseline Stage 35 Configuration}

In this study NASA Stage 35 is used as the baseline configuration. NASA Stage

35 is a transonic inlet stage for a core compressor, and has been widely used by several researchers. The stage has a design pressure ratio of 1.82 at a mass flow of $20.19 \mathrm{~kg} / \mathrm{sec}$ and has a rotor tip speed of $455 \mathrm{~m} / \mathrm{sec}$. This stage was originally designed and tested at NASA Glenn Research Center by Reid and Moore ${ }^{69}$. All calculations reported in this section were made at 100 percent design speed.

A computational grid with five blocks: an H-block upstream, C-blocks around the rotor and the stator, and O-blocks for the tip clearance and stator hub clearance is used. Tip clearance grids had between 9-11 points across the gap. The full grid had approximately 1.13 million points. Figure 4.12 shows the grid used for the NASA Stage 35 , and figure 4.13 shows a close-up view of the rotor tip clearance grid.

An updated version of the k- $\omega$ SST turbulence model is used to model the turbulent flow for this calculation. Solutions for every operating point are obtained after approximately 2500 iterations (70 minutes) on a Pentium $42.80 \mathrm{GHz} 512 \mathrm{MB}$ PC. These Stage 35 results represent the validation for the recent modifications made to the SWIFT code. The previous version of k- $\omega$ SST turbulence model predicted higher turbulent viscosities that made the boundary layers more resistant to separation due to errors in the upwind scheme.

The performance map computed for the baseline Stage 35 geometry was compared with the experimental data obtained by Reid and Moore. In the map, as shown 
in figure 4.14, the total pressure ratio of the stage is plotted as function of the ratio of the mass flow rate to the choking mass flow rate of the respective configurations. As stated earlier, it is customary that mass flow is normalized so as to avoid distortion between the relative shapes of the curves and removing the differences in the computed choking mass flow rate as factor in the design process.

The choking mass flow rate for the Stage 35 configuration was computationally found to be $20.91 \mathrm{~kg} / \mathrm{sec}$; experimental choking mass flow rate is reported as 20.95 $\mathrm{kg} / \mathrm{sec}$. The figure shows very good agreement with the experimental values, although the stall point was not captured accurately. 


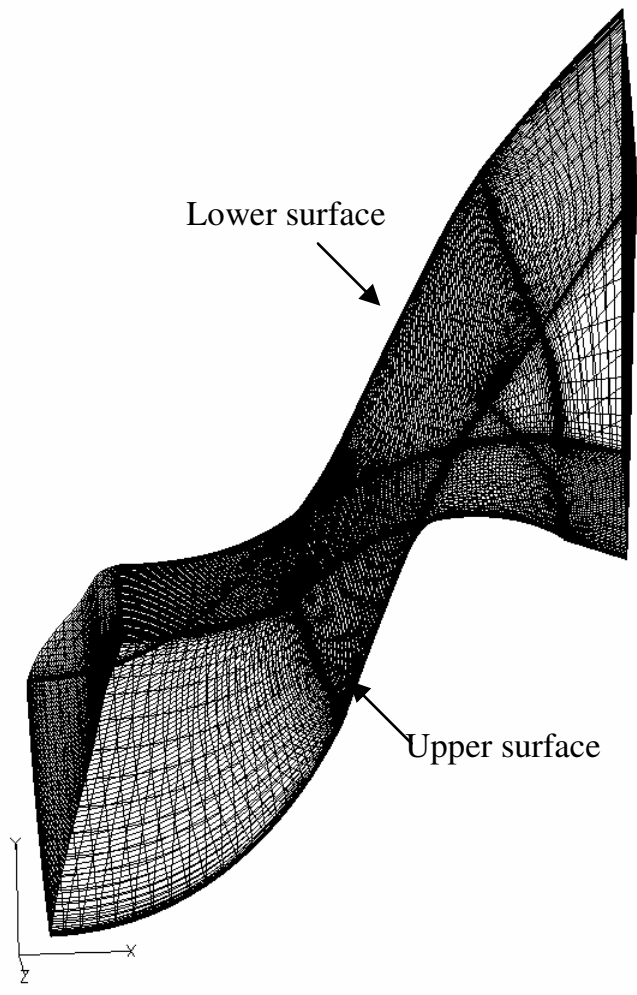

Figure 4.1: A nominal H-type grid for the Rotor 67 configuration.

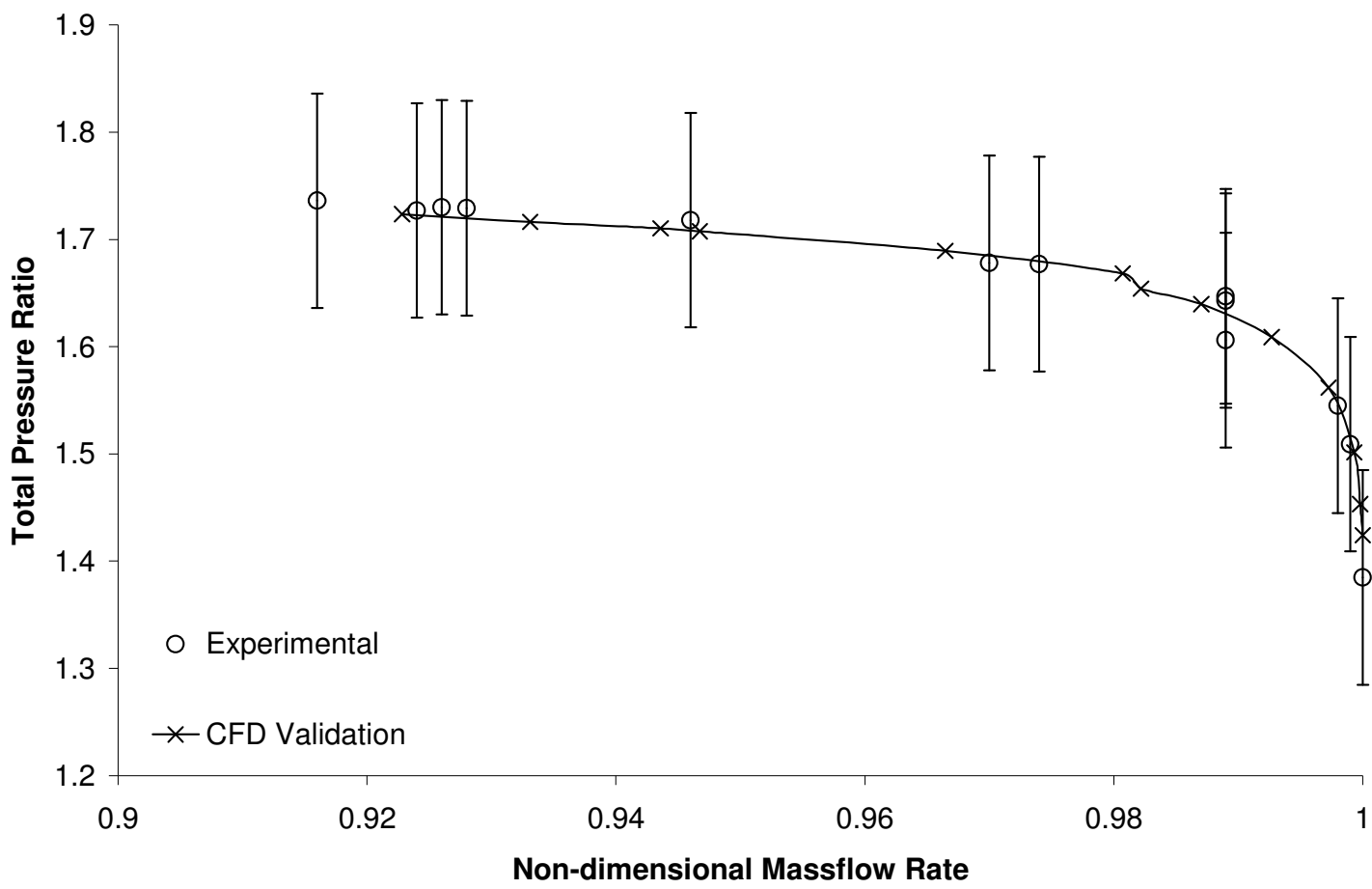

Figure 4.2: Characteristic performance map validating the CFD with the experimental results for Rotor 67 configuration. 


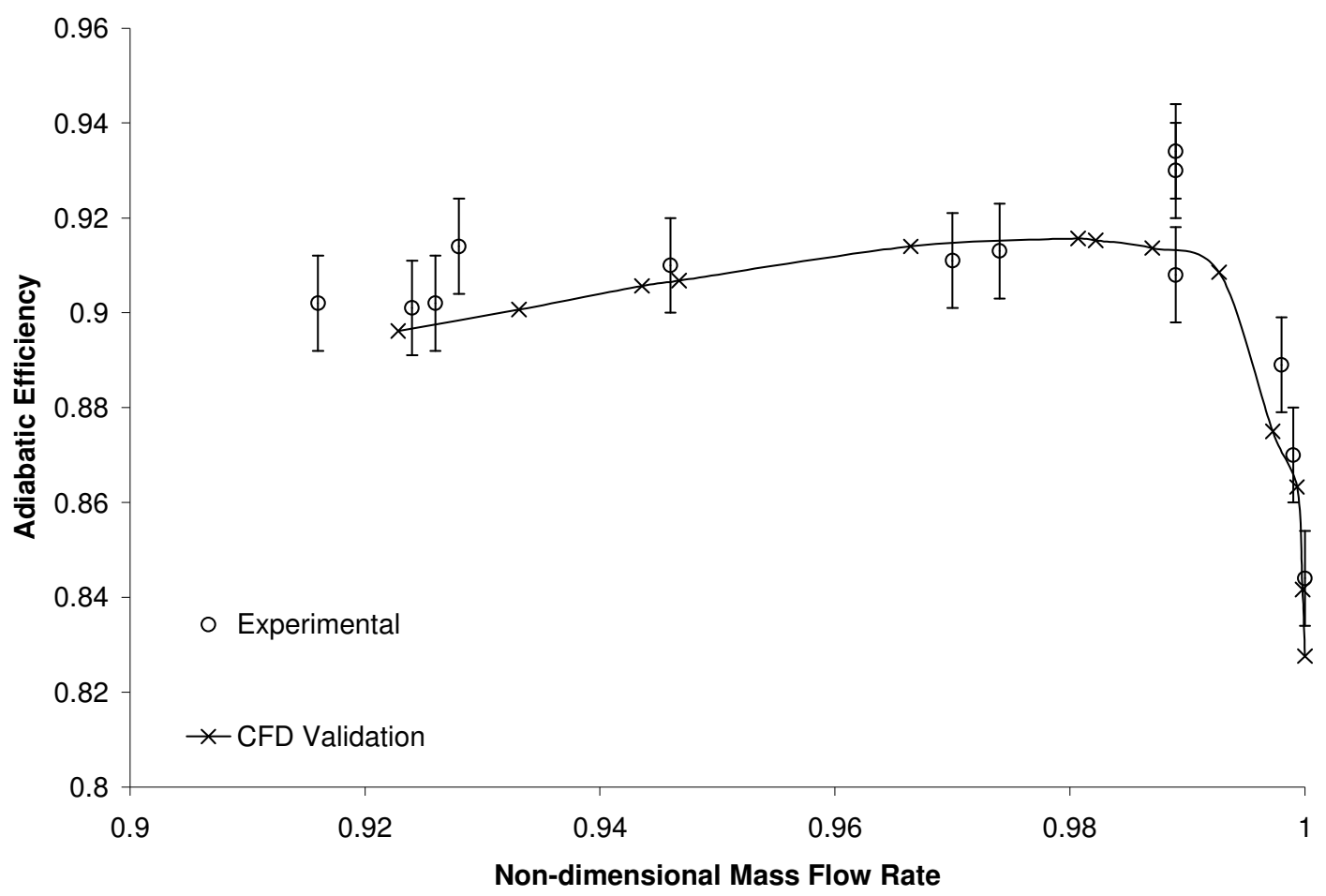

Figure 4.3: Characteristic efficiency map validating the CFD with the experimental results for Rotor 67 configuration.

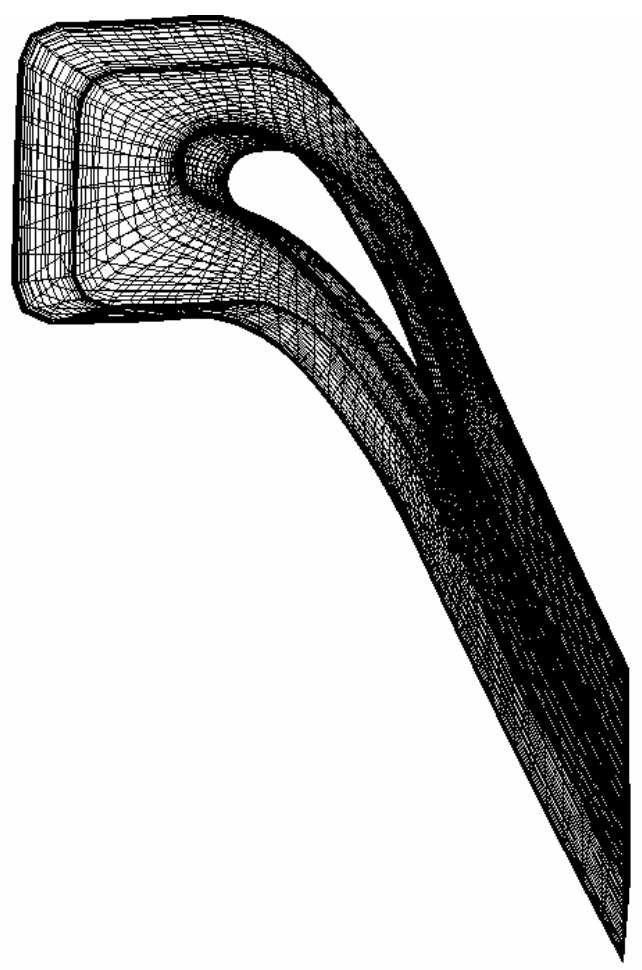

Figure 4.4: C-type computational grid for the Goldman Turbine Vane Configuration. 


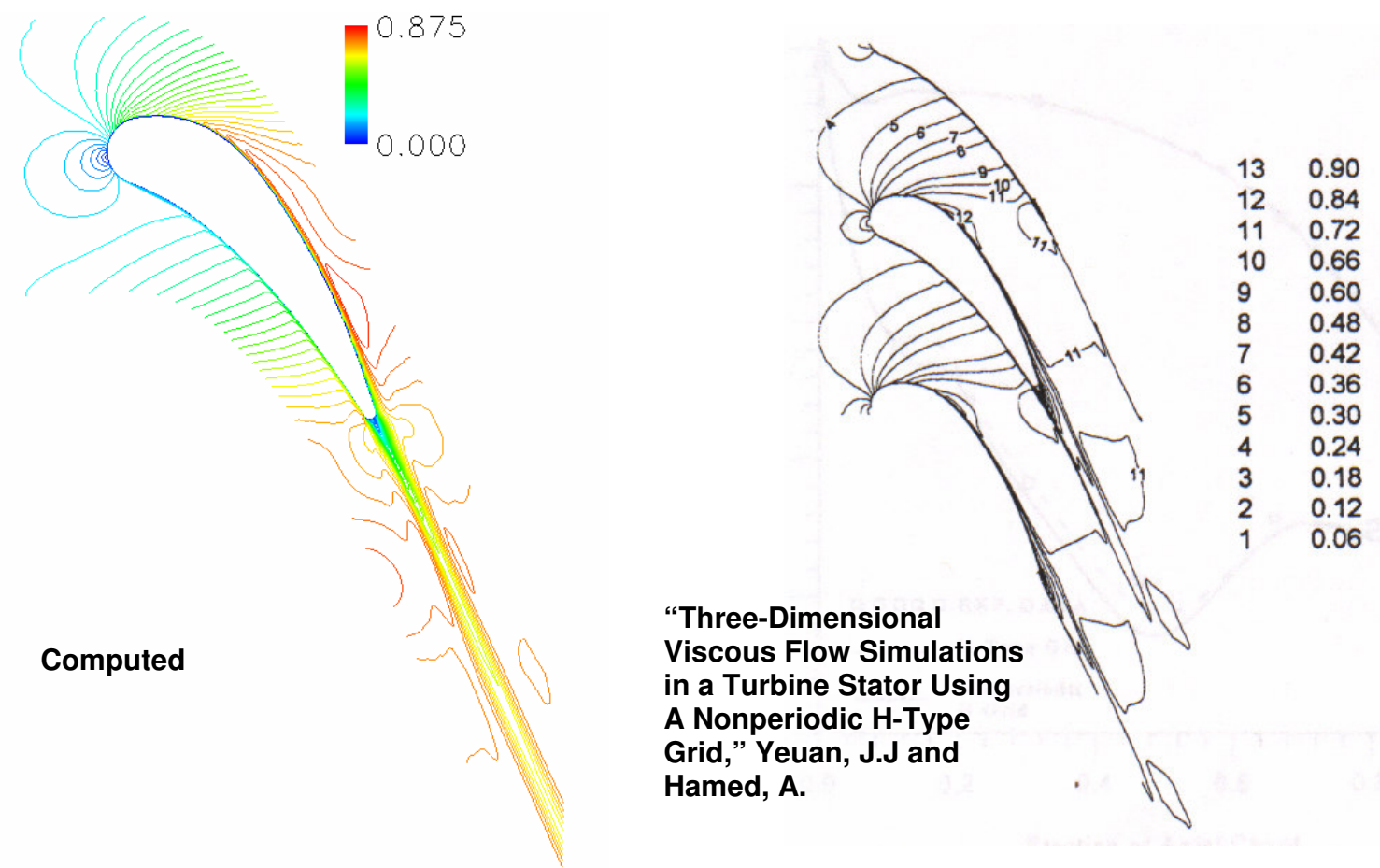

Figure 4.5: Mach number contour comparison between CFD and published data at mid-span location for Goldman Turbine Vane.

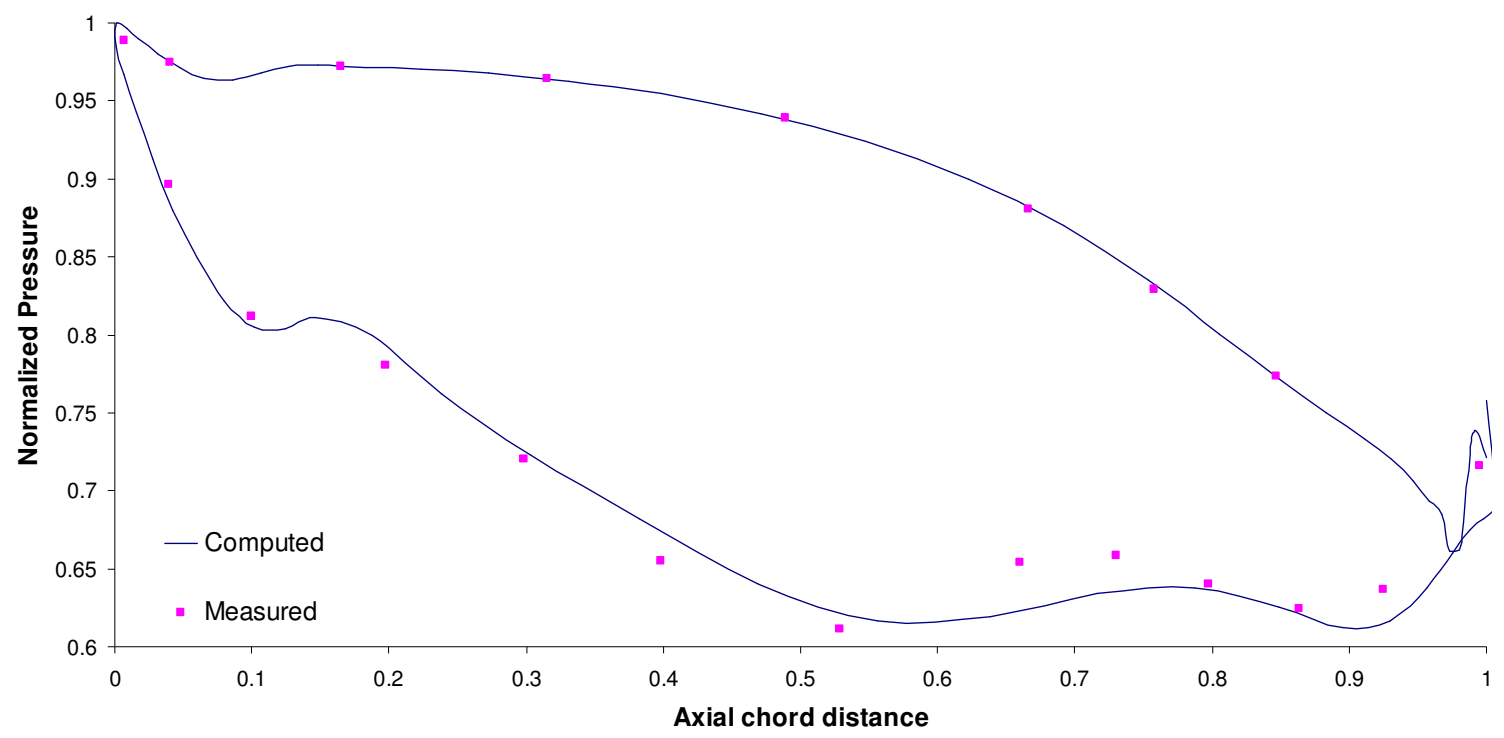

Figure 4.6: Comparisons between the experimental and CFD blade surface pressure distribution for the Goldman Turbine Vane. 


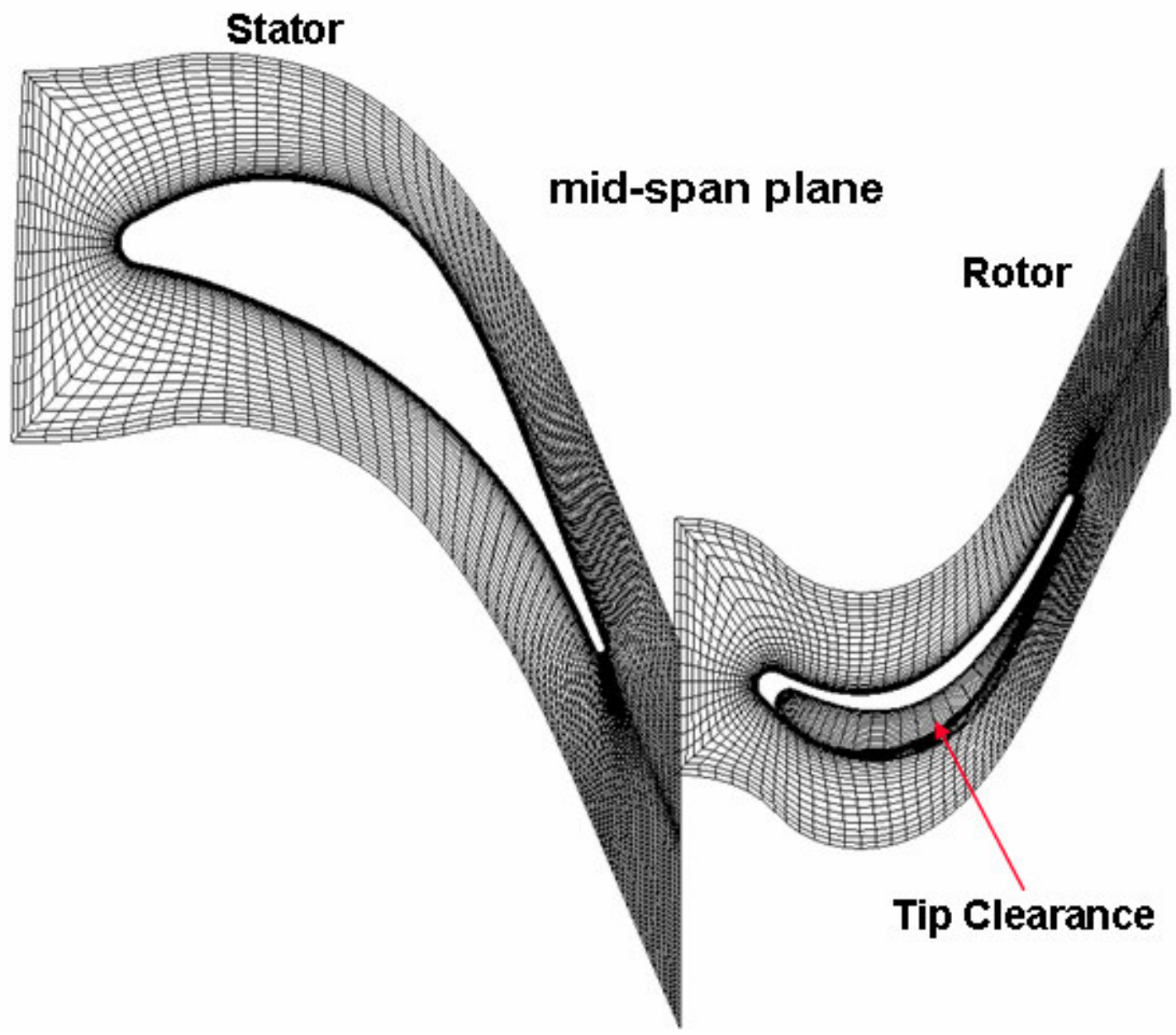

Figure 4.7: Sectional view of the computational grid used for SSME Turbine stage analysis.

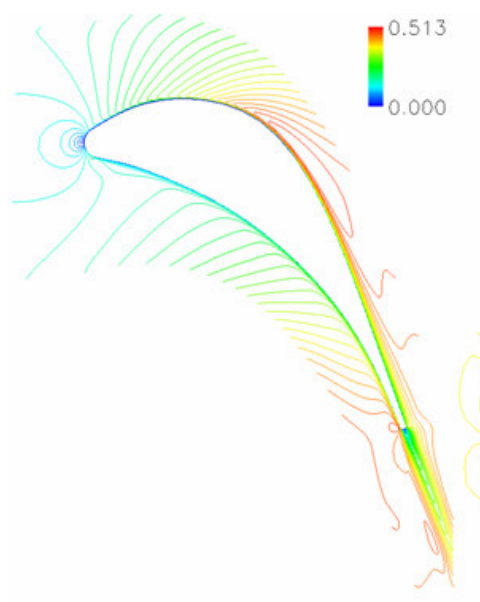

Validated results

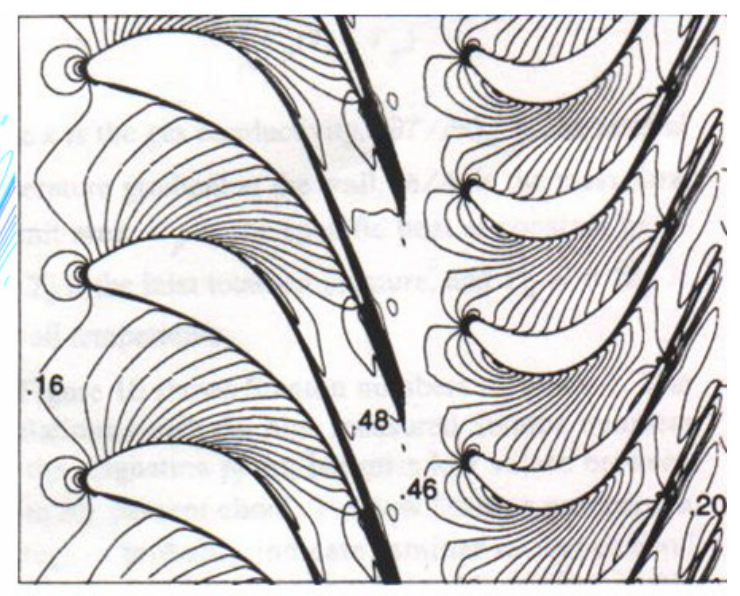

From: "Calculations of Multistage Turbomachinery Using Steady Characteristic Boundary Conditions", Chima R.V. (1998)

Figure 4.8: Mach number contours comparison between published data and CFD at mid-span location for the SSME Turbine stage. 


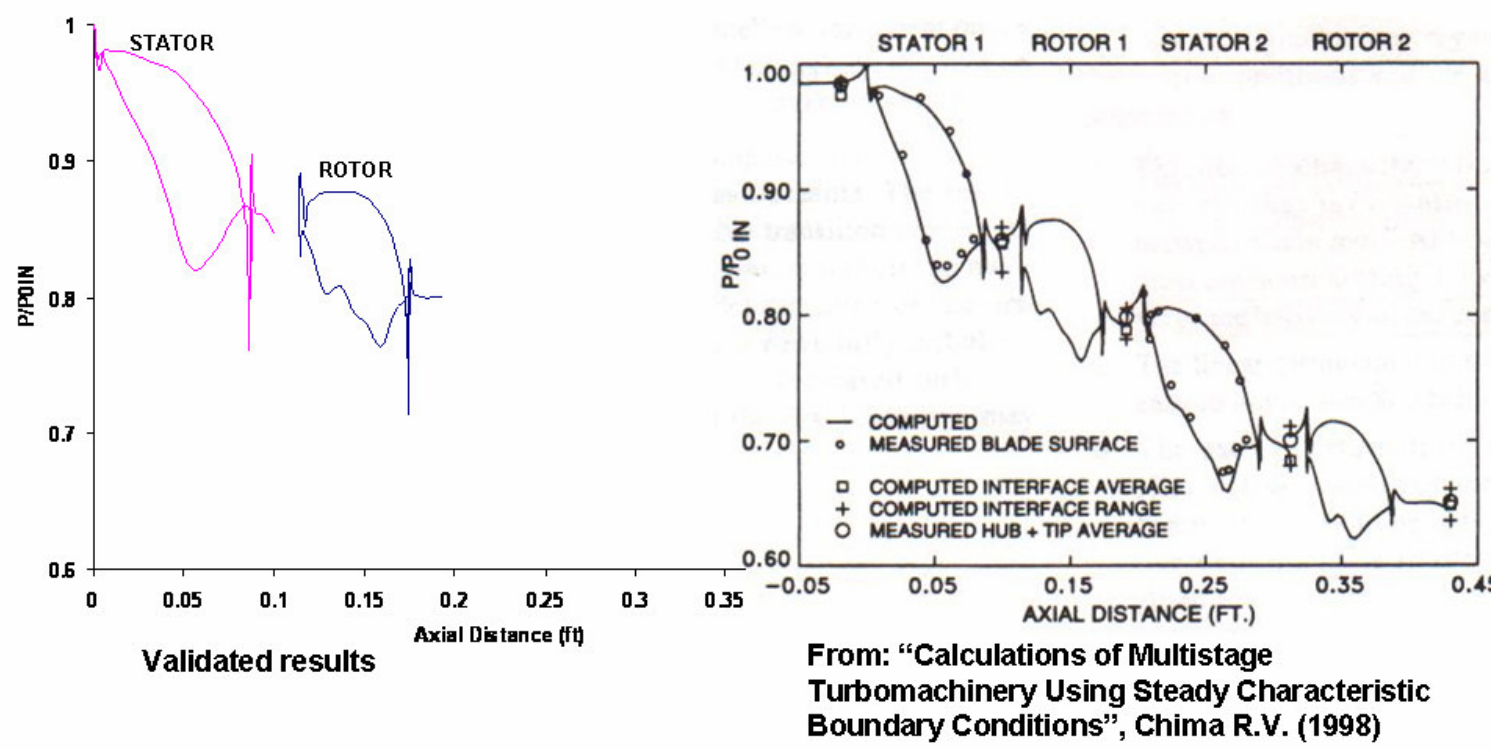

Figure 4.9: Comparisons between previously published and CFD reproduced normalized pressure on blade surface for the SSME Turbine stage.

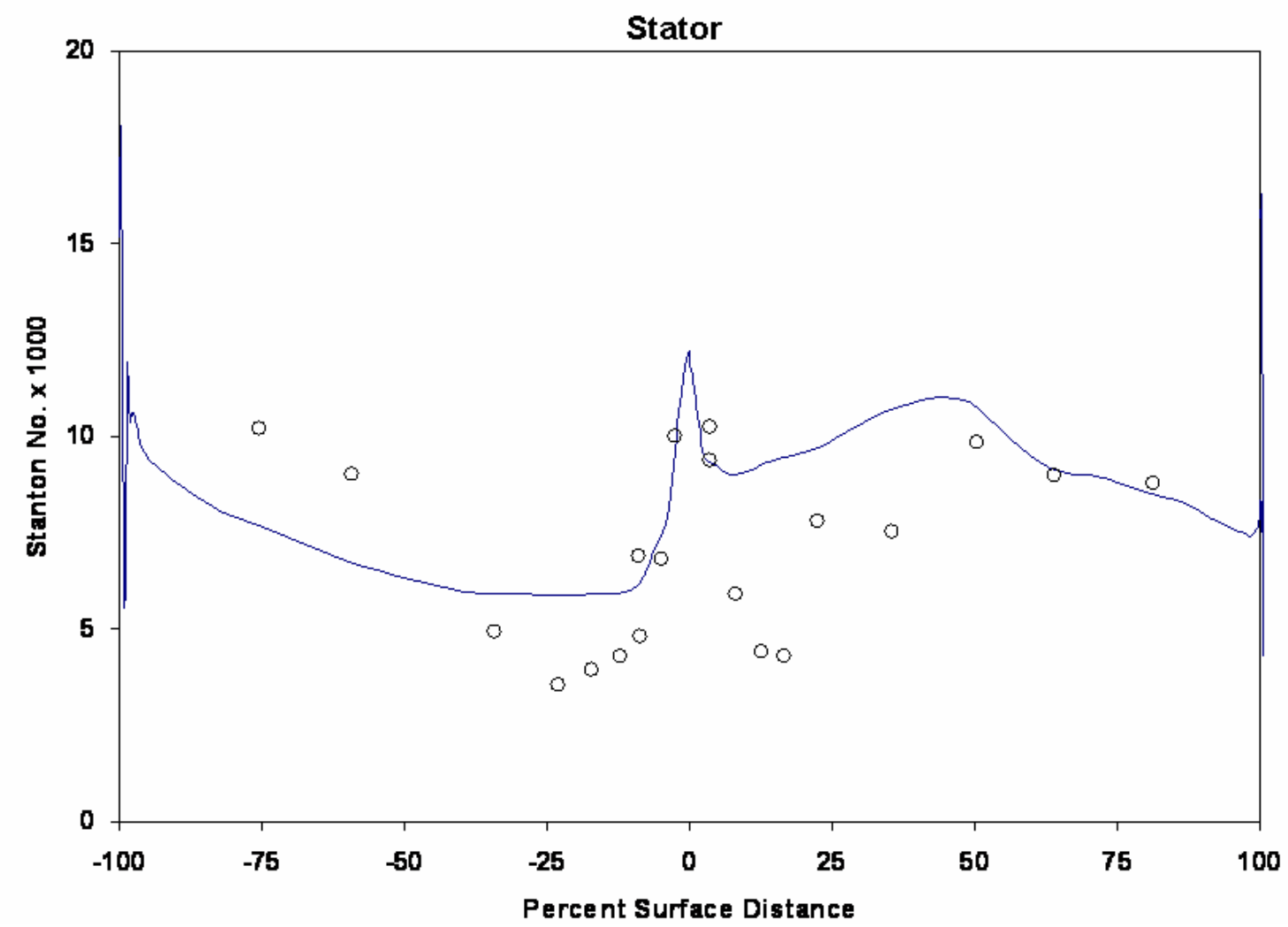

Figure 4.10: Comparisons between experimental and computed Stanton number on stator blade surface for the SSME Turbine stage. 


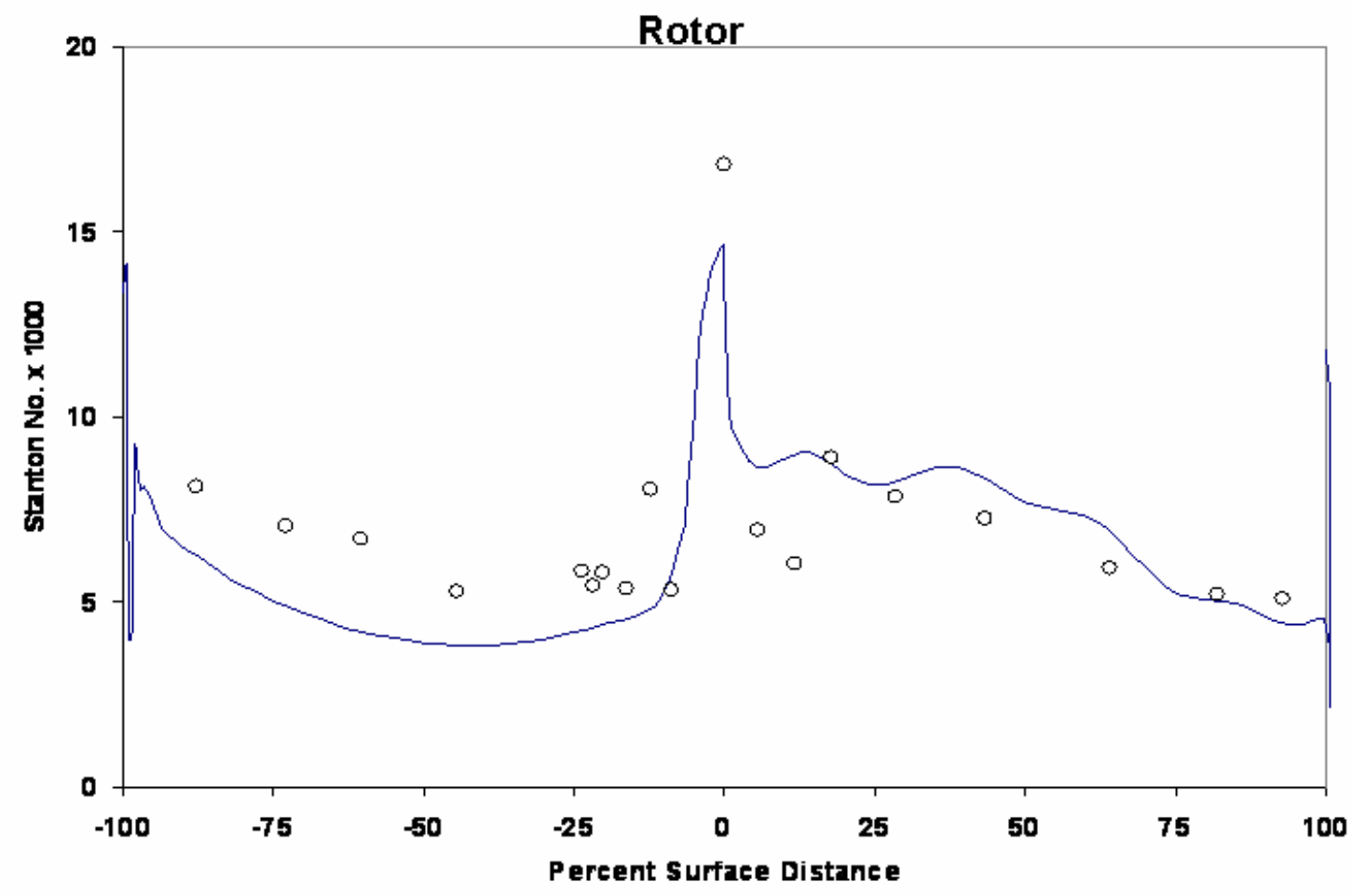

Figure 4.11: Comparisons between experimental and computed Stanton number on rotor blade surface for the SSME Turbine stage. 


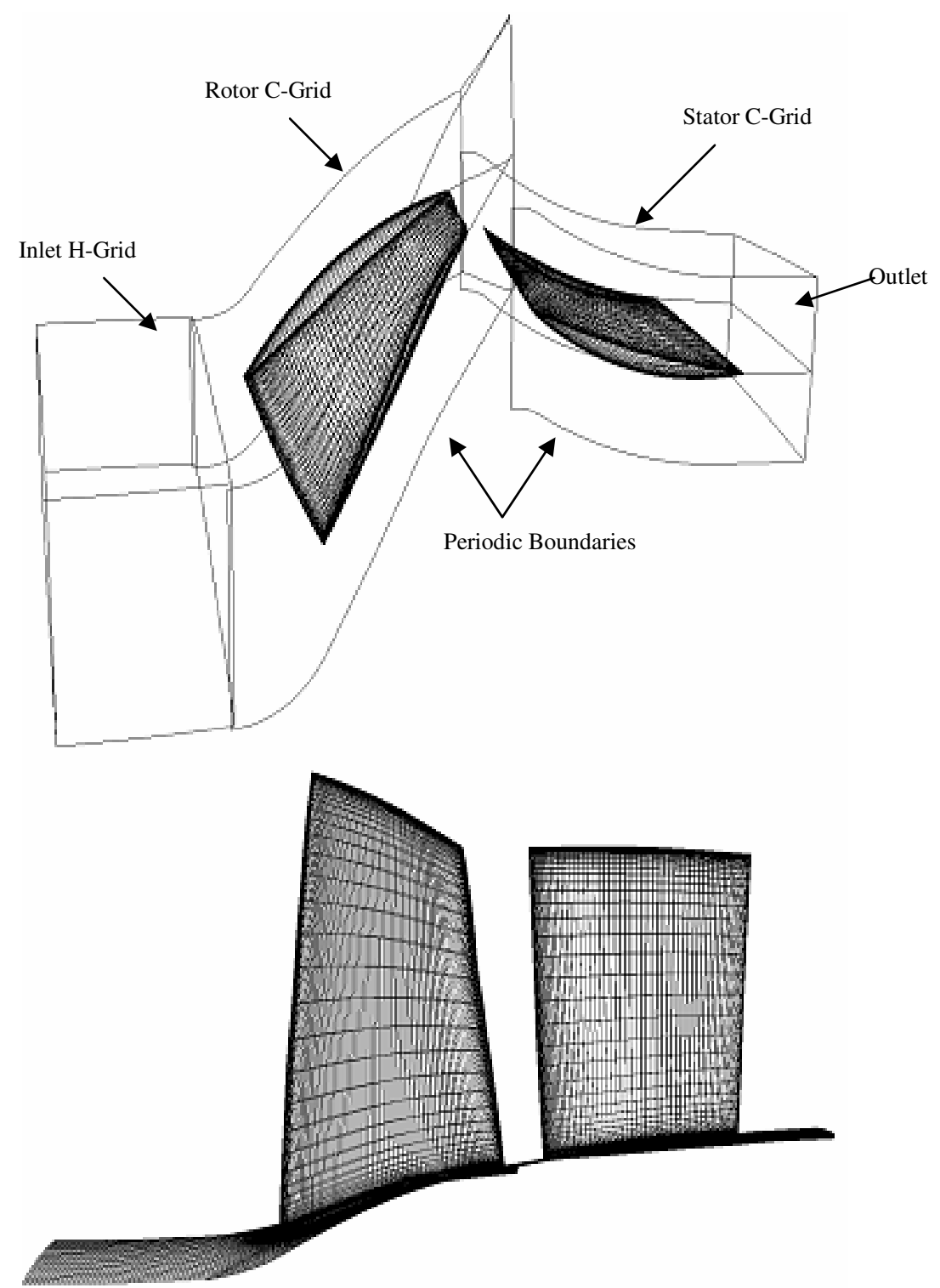

Figure 4.12: Stage 35 multi-block grid used in the simulations.

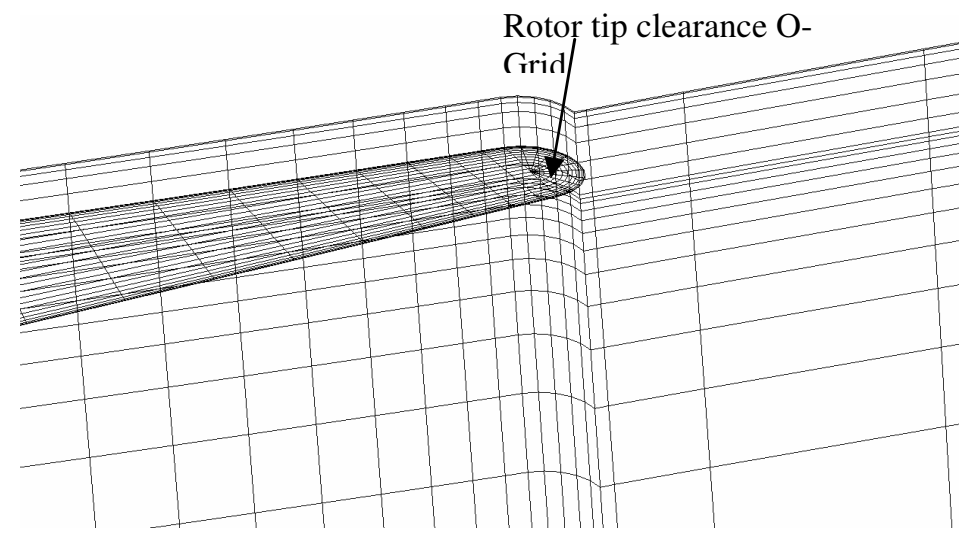

Figure 4.13: Rotor tip clearance grid for Stage 35. 


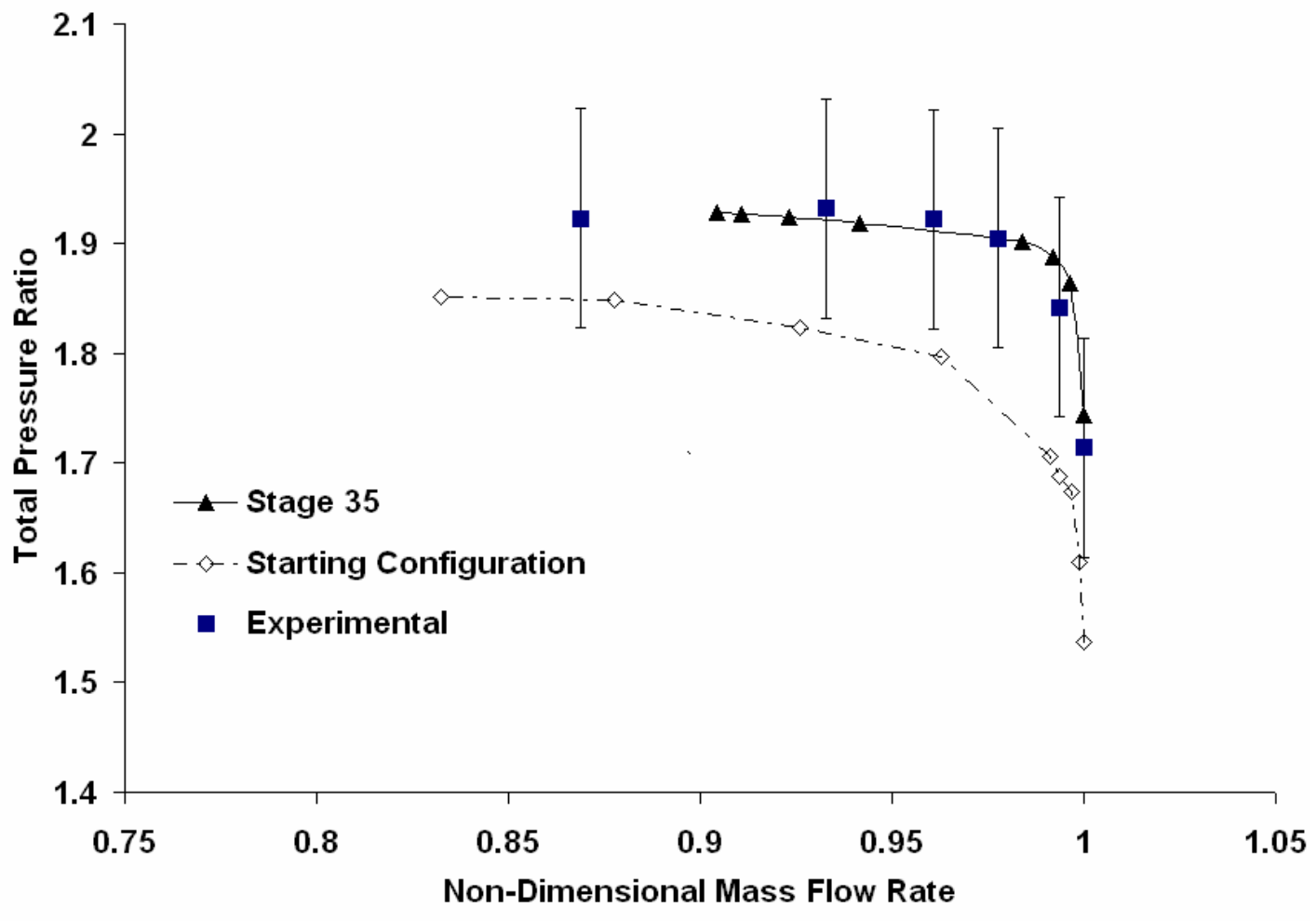

Figure 4.14: Characteristic performance map comparing Starting Configuration with the original Stage 35 configuration and the experimental data. 


\section{CHAPTER V}

\section{RESULTS I: PRELIMINARY APPLICATION OF METHODOLOGY}

This chapter will present the results for the preliminary application performed using the newly developed design and optimization methodology. A proof of concept study will be performed wherein the design tools and CFD tools associated with the methodology will be evaluated. The purpose of this section is to demonstrate the effectiveness of the methodology. A smaller set of design variables will be selected to assess the effects of blade topology changes on the blade aerodynamic performance. An assessment of the modifications to the shock structure and flow blockages due to the changes in design variables will provide a way to carefully select the design variables for

future studies. The preliminary application of the methodology also provides a way to investigate the effects of the design tools on compressor stage design before a detailed application is considered. The design methodology validated in this chapter act will be built upon in the comprehensive application of the methodology presented in Chapter 6.

Description of the design variables selected along with details of the optimization loop is summarized. The design methodology is applied to a 'starting configuration' and then the 'starting configuration' is optimized to give a 'preliminary configuration'. Results are presented for the performance maps first, followed by an analysis of the flow physics to interpret the results. 


\subsection{Design Variables}

Eight design variables- four (leading edge and trailing edge mean camber line angle at hub and tip) in case of the rotor and four (leading edge and trailing edge mean camber angle at hub and tip) for the stator- are chosen for this study. Each of the eight design variables are changed systematically within a user-specified range while all the other parameters previously described are unchanged. By keeping the other variables unchanged and changing only the design variables, consistency is achieved. This section is a demonstration of a new approach rather than a comprehensive design analysis; hence the selection of four design variables for each blade is justifiable. A more comprehensive application the methodology where more design variables are considered will be presented subsequently. Figure 5.1 shows design variables used for this concept proof.

\subsection{Comparison between Stage35 and Starting Configuration}

A parametric approximation close to the Stage 35 configuration was constructed using CCGEOM. In this approximation procedure the blade sections at the hub, mid-span and tip were designed independently, but with a view to match the mid-span section as closely to the best configurations (Stage 35) as feasible. This approximated configuration is described as the 'starting configuration' throughout this study.

Although the intent was to match the Stage 35 geometry as closely as possible, some differences were present between the Stage 35 and the starting configuration. Even 
though CCGEOM is a good parametric design tool, accurately matching the complex compressor blade designs is a challenging task. For instance, the maximum blade thicknesses at the rotor hub could not be accurately matched. Transonic rotors are very sensitive to blockage effects due to small changes to the blade thickness, and the smallest of modifications can result in some discrepancies.

Figure 5.2 for the rotor blade and figure 5.3 for the stator blade, show comparisons between the Stage 35 and starting configuration at hub, mid-span and tip locations. From both figures it becomes apparent that the mid-span section is matched very closely. For the rotor, at the hub the starting configuration has larger maximum thickness than the Stage 35. At the tip the starting configuration has higher leading edge incidence angles.

By comparing the performance map for the starting configuration to the Stage 35 (see Figure 4.14) it can be observed that the starting configuration exhibits approximately $4 \%$ decrease in total pressure ratio over the entire operating regime. The operating range for the Stage 35 is shorter than that for the starting configuration. The choking mass flow rate for the starting configuration was found to be $19.9 \mathrm{~kg} / \mathrm{sec}$. It may be noted that changing the geometry changes the frontal area, changing the choking mass flow rate. As mentioned earlier, the increased thickness can increase the blockage effects and hinder the performance of the starting configuration. Hence the operating range and the total pressure ratio could not be accurately captured. 


\subsection{Optimization Results}

Here, the optimization was performed for aerodynamic benefits only. As stated earlier in Chapter 3, a design of experiments approach was used to select from 81 combinations of the 8 design variables, and the response surfaces for the adiabatic efficiency and total pressure ratio were constructed The automated optimizer was subsequently executed with an intent to maximize a weighted average of adiabatic efficiency, and total pressure ratio and determine the optimized configuration. The optimum values for the eight design parameters considered are given in tables 5.1 (rotor) and 5.2 (stator). The blade section constructed from the optimum values are referred to as the 'preliminary configuration' for the remainder of the study. Figure 5.4 for the rotor blade and figure 5.5 for the stator blade, show comparisons between the preliminary and starting configuration at hub, mid-span and tip locations. For the stator blade, the tip section for the preliminary configuration has a higher leading edge incidence angle. For the rotor blade, significant differences can be noticed at the hub and tip leading edge where the preliminary profile has higher leading edge incidence angle. Consequences of these differences on the stage performance will be discussed subsequently.

A comparison between the design values predicted by the RSE's and computed value by the CFD analysis for the two objective functions are given in table 5.3. 


\subsubsection{Performance map comparisons between Starting and Preliminary Configurations}

Performance maps for the starting configuration and the preliminary design were obtained, and are shown in figure 5.6. For the optimized configuration, the choking point was found to be $20.0 \mathrm{~kg} / \mathrm{sec}$, compared to $19.9 \mathrm{~kg} / \mathrm{sec}$ for the starting configuration. On comparing the preliminary configuration to the starting configuration, it can be noticed that the preliminary case exhibits approximately $1.3 \%$ improvement in total pressure ratio. The operating range for the preliminary configuration is only slightly lesser $(0.015 \%)$ than the starting configuration. The optimization was performed at the design mass flow rate and hence does not guarantee that the stall point will occur at exactly the same mass flow rate as the starting configuration. One way to account for the stall point and the operating range would be to perform the optimization at multiple points on the performance map or by taking a mass average of the entire operating range in the optimization loop.

Figure 5.7 shows the adiabatic efficiency map for the two configurations. Clearly, it can be interpreted from the efficiency map that the difference in peak adiabatic efficiency between the two configurations is significant. Near choke conditions, the preliminary configuration has a $1.5 \%$ higher peak efficiency than the starting configuration. The higher peak efficiency close to design point exhibited by the preliminary configuration makes it an appealing option over the starting configuration.

A plot comparing the rise in total temperature for the starting configuration with the preliminary configuration is shown in figure 5.8. It can be observed that the total 
temperature ratio for the preliminary configuration shows a slight $(0.8 \%)$ increase when compared to the starting configuration.

From this increase in total pressure ratio and peak adiabatic efficiency for the preliminary configuration over the starting configuration without a decrease to the operating range, it may be concluded that the preliminary configuration is an improvement over the starting configuration.

\subsubsection{Flowfield comparisons between Starting and Preliminary Configurations}

Initial analysis suggests that the loading on the compressor blade has been increased. This may be achieved by either a weakening of the blade-to-blade shock structure on the rotor or the stator, or by minimizing the secondary flow interactions. In order to fully understand the reasons behind the predicted performance improvement of the preliminary shape, an examination of the flow structure was done. The inspection of the flow field provides us with a means to quantify the differences based on the changes made to blade sections in the optimization process. Figure 5.9 shows the blade-to-blade passage for a blade along with a description of span and chord. For the entirety of the study the design point corresponds to the peak efficiency point, which is approximately $97 \%$ of the choking mass flow rate and off-design condition corresponds to $87 \%$ of the choking mass-flow rate.

Figure 5.10 compares the entropy contours at mid-passage in the meridional plane

for the starting and preliminary configurations at an off-design condition $\left(0.87 m_{\text {choke }}\right)$. It 
is seen that a region of high entropy exists at approximately $30 \%$ chord near in the tip of the blade for both configurations (starting and preliminary). This high entropy is attributed to the tip clearance vortex, which will be discussed again subsequently. It can be noticed that the casing boundary layer is separated near the rotor leading edge because of the shock and the clearance vortex interactions. By comparing the two configurations, it is clearly observed that the separation is less pronounced for the preliminary configuration.

Figure 5.11 compares the off-design condition $\left(0.87 m_{\text {choke }}\right)$ entropy contours for the starting and preliminary configurations at tip clearance section in the blade-to-blade plane. It should be noted that although the analysis was done for a single C-grid rotorstator configuration, the results going forward have been periodically rotated to reflect two C-grid passages or one entire blade to blade passage for better visible clarity. On figure 5.11a, three areas are marked as A- ahead of the blade, B- $30 \%$ chord location above the blade and C- mid passage wake region. Comparing these three areas between figure 5.11a and 5.11b, it can be noticed that the magnitude of entropy at all three areas is lower for the preliminary configuration compared to the starting configuration. This indicates that the blockages both upstream and downstream of the rotor blade are reduced. This, as expected, has a substantial influence on the performance of the rotor especially at off-design conditions.

It was mentioned before that the rotor for preliminary configuration has a higher leading edge incidence angle as compared to the starting configuration. Although the 
higher incidence angles can cause the flow to accelerate more than before and result in a stronger shock, this phenomenon was not observed for the preliminary design.

Mach number contours comparing the starting and preliminary configuration at $90 \%$ span location from the hub for design condition $\left(0.97 m_{\text {choke }}\right)$ is shown on figure 5.12. Two areas, A- rotor leading edge and B- stator trailing edge are shown on figure 5.12a. By comparing the Mach number at the rotor leading edge (point A) it is apparent that the strength of the shock from the suction side of the blade for preliminary configuration (figure 5.12b) has been reduced when compared to the starting configuration. The Mach numbers in the vicinity of the stator trailing edge (point B) are much higher for the preliminary configuration, resulting in an increase in the overall loading of the stator blade.

Mach number contours in the blade-to-blade plane at $90 \%$ span comparing the starting to the preliminary configuration at an off-design condition $\left(0.87 m_{\text {choke }}\right)$ are shown in figure 5.13. One obvious noticeable difference between the two configurations is at the rotor leading edge. In figure 5.13a smeared shock emerging from the pressure surface leading edge extends over the entire blade-to-blade passage (marked as A) and hence interacts with the next blade at about $60 \%$ chord distance from the leading edge. This interaction adversely affects the flow on the next blade, causing the boundary layer to thicken and possibly separate. For the preliminary configuration on the other hand, this interaction does not exist as the strong leading edge shock emerging from the pressure surface now appears as a weaker detached shock. The detached shock loses its strength 
before it can reach the neighboring rotor blade and hence its interaction with the next blade and its boundary layer is found to be minimal.

Figure 5.14 shows the effects of the shock and clearance flow on the casing boundary layer. A blade-to-blade plot of Mach number contours for starting (Figure 5.14a) and preliminary configuration (Figure 5.14b) at the blade tip- off design condition is shown at the right of the figure. This close to the casing the shock is highly smeared, and the tip vortex can be followed through the shock to the pressure side of the neighboring blade. Two meridional plots of Mach number contours above 70 percent span at two tangential locations (20\% and 40\%) are shown on the left. The leading edge and trailing edges are also shown for reference.

In figure 5.14a, the bottom left plot is near the suction surface of the blade shows the clearance vortex just downstream of the leading edge, followed by a region of lowspeed flow. The shock is evident at near $50 \%$ chord, followed by an even larger region of low-speed flow caused by the shock-boundary layer interaction. At $40 \%$ tangential location the clearance vortex is evident at approximately $30 \%$ chord. The dotted arrow shows the direction of the vortex in the tangential direction. The solid arrow indicates the chordwise distance of the clearance vortex from the leading edge.

For the preliminary configuration, it is observed from figure $5.14 \mathrm{~b}$ that the tangential direction of the clearance vortex is slightly different. The tip vortex for the preliminary configuration appears to be traveling at higher angle relative to the normal when compared to figure 5.14a. Also the chordwise position of the same clearance vortex 
is at a greater chordwise location ( 40\%), which is again indicated by the longer solid arrow with leading edge as the reference.

In the blade-to-blade plane on the right side of figure 5.14a, a strong shock emerging from the suction side of blade leading edge is evident for the starting configuration. Comparing this shock to its counterpart in the preliminary configuration in figure $5.14 \mathrm{~b}$, it is noticed that this shock for the latter is at a lower angle relative the blade chord. The lower shock angle in case of the preliminary configuration implies that the strength of this suction surface shock is lower relative to its starting configuration counterpart. It is noticed again that the interactions between the shock emerging from pressure side and the neighboring blade are lower for the preliminary configuration compared to the starting one.

It is logical to state that the better alignment of the flow near the tip section for both rotor and stator can inherently reduce the strength of the tip clearance vortex; this was observed in the entropy contours for the preliminary configuration discussed before in figure 5.10. The change in blade topology at tip also modifies the direction of the clearance vortex. These aerodynamic changes are beneficial for the performance of the compressor stage. In this case the blockage modifications and reduced shock strengths result in increased total pressure ratios across the stage for the preliminary configuration when compared to the starting configuration. No additional penalty in the form of decrease in adiabatic efficiency or loss in operating range was observed. 


\begin{tabular}{|c|c|c|c|}
\hline $\begin{array}{c}\text { Hub L.E } \\
\text { (Degrees) }\end{array}$ & $\begin{array}{c}\text { Hub T.E } \\
\text { (Degrees) }\end{array}$ & $\begin{array}{c}\text { Tip L.E } \\
\text { (Degrees) }\end{array}$ & $\begin{array}{c}\text { Tip T.E } \\
\text { (Degrees) }\end{array}$ \\
\hline $\mathbf{4 8 . 2 2}$ & $\mathbf{1 2 . 8 1}$ & $\mathbf{6 8 . 0 1 1}$ & $\mathbf{4 4 . 2 1}$ \\
\hline
\end{tabular}

Table 5.1: Optimized values of rotor design variables used in preliminary application of methodology.

\begin{tabular}{|c|c|c|c|}
\hline $\begin{array}{c}\text { Hub L.E } \\
\text { (Degrees) }\end{array}$ & $\begin{array}{c}\text { Hub T.E } \\
\text { (Degrees) }\end{array}$ & $\begin{array}{c}\text { Tip L.E } \\
\text { (Degrees) }\end{array}$ & $\begin{array}{c}\text { Tip T.E } \\
\text { (Degrees) }\end{array}$ \\
\hline $\mathbf{4 7 . 8 6}$ & $\mathbf{5 . 0 8}$ & $\mathbf{3 2 . 2 3}$ & $\mathbf{- 1 0 . 4 1}$ \\
\hline
\end{tabular}

Table 5.2: Optimized values of stator design variables used in preliminary application of methodology.

\begin{tabular}{|c|c|c|}
\hline & $\begin{array}{c}\text { Total } \\
\text { Pressure } \\
\text { Ratio }\end{array}$ & $\begin{array}{c}\text { Adiabatic } \\
\text { Efficiency }\end{array}$ \\
\hline Design & $\mathbf{1 . 8 1 5 6}$ & $\mathbf{. 8 2 0 4}$ \\
\hline CFD & $\mathbf{1 . 8 1 1 4}$ & $\mathbf{. 8 2 0 9}$ \\
\hline \% Diff & $\mathbf{. 4 2}$ & $\mathbf{. 1 1}$ \\
\hline
\end{tabular}

Table 5.3: Differences observed between predicted and computed values for the objective functions in preliminary application of methodology. 


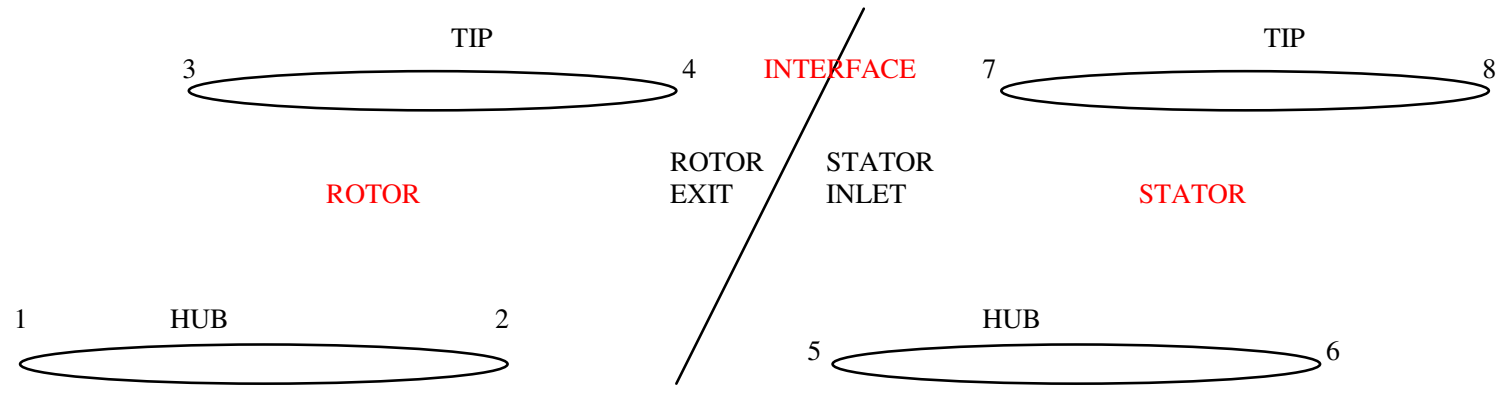

Figure 5.1: Design variables (camber angles) used for the preliminary application of methodology. 


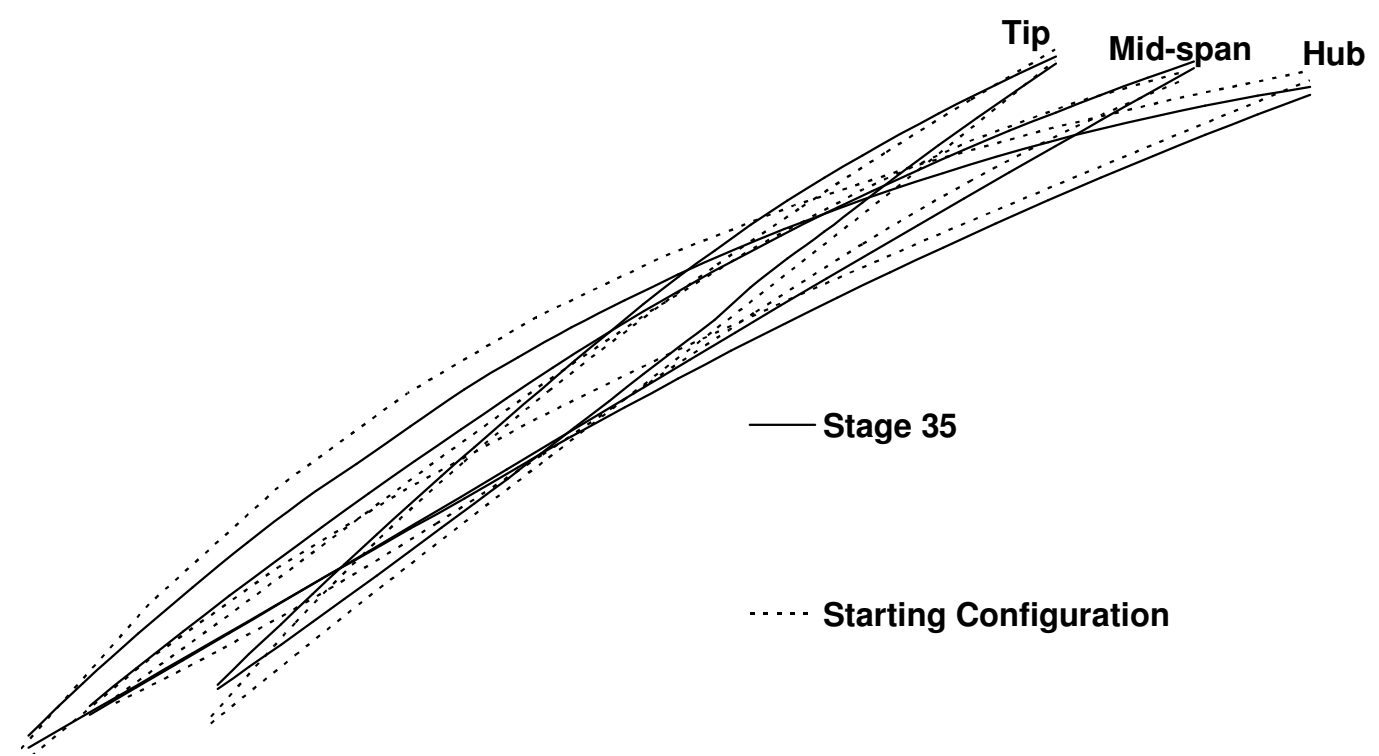

Figure 5.2: Rotor blade profile comparisons between the Stage 35 and starting configurations at hub, mid-span and tip locations.

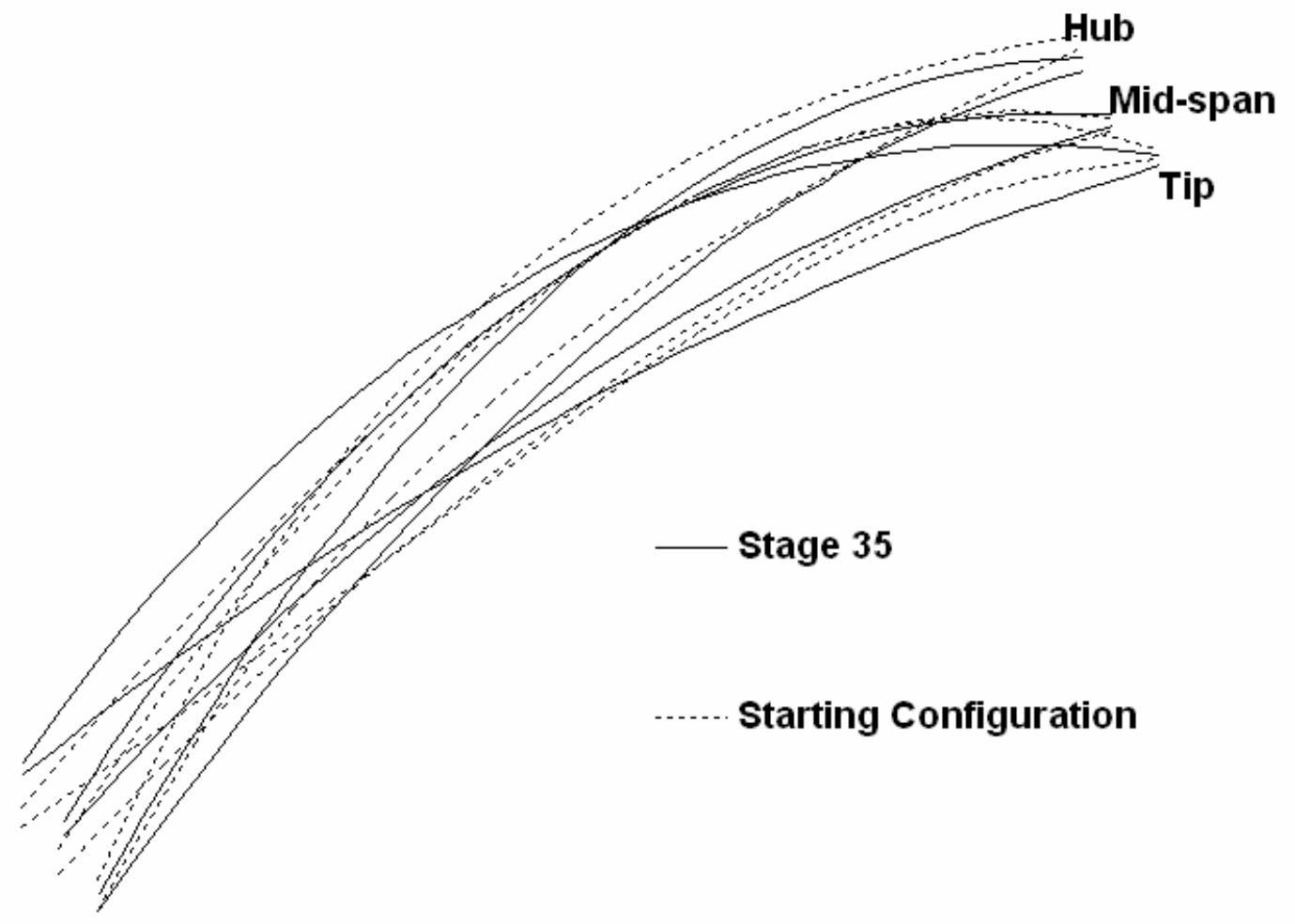

Figure 5.3: Stator blade profile comparisons between the Stage 35 and starting configurations at hub, mid-span and tip locations. 


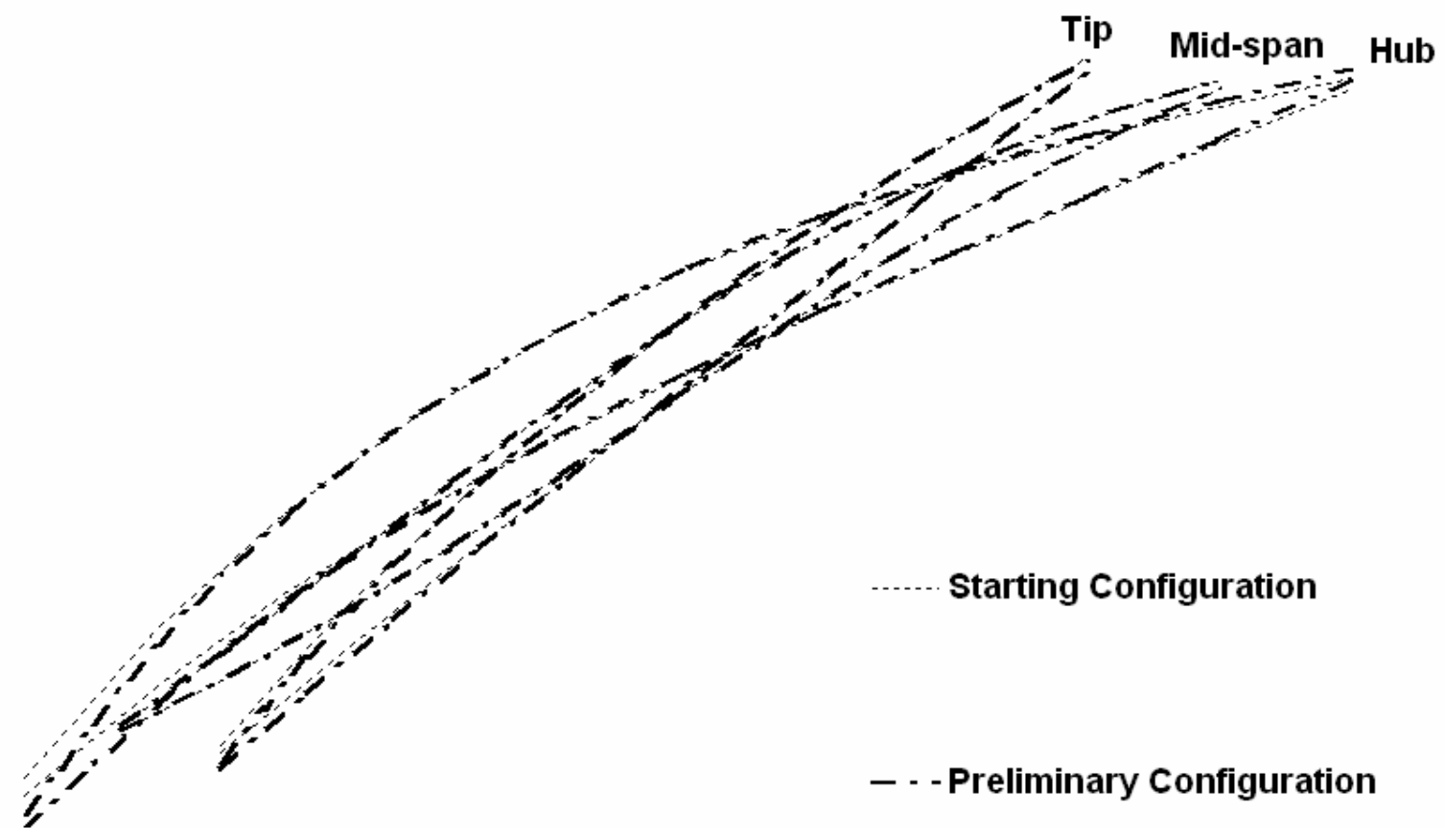

Figure 5.4: Rotor blade profile comparisons between the starting and preliminary configurations at hub, mid-span and tip locations.

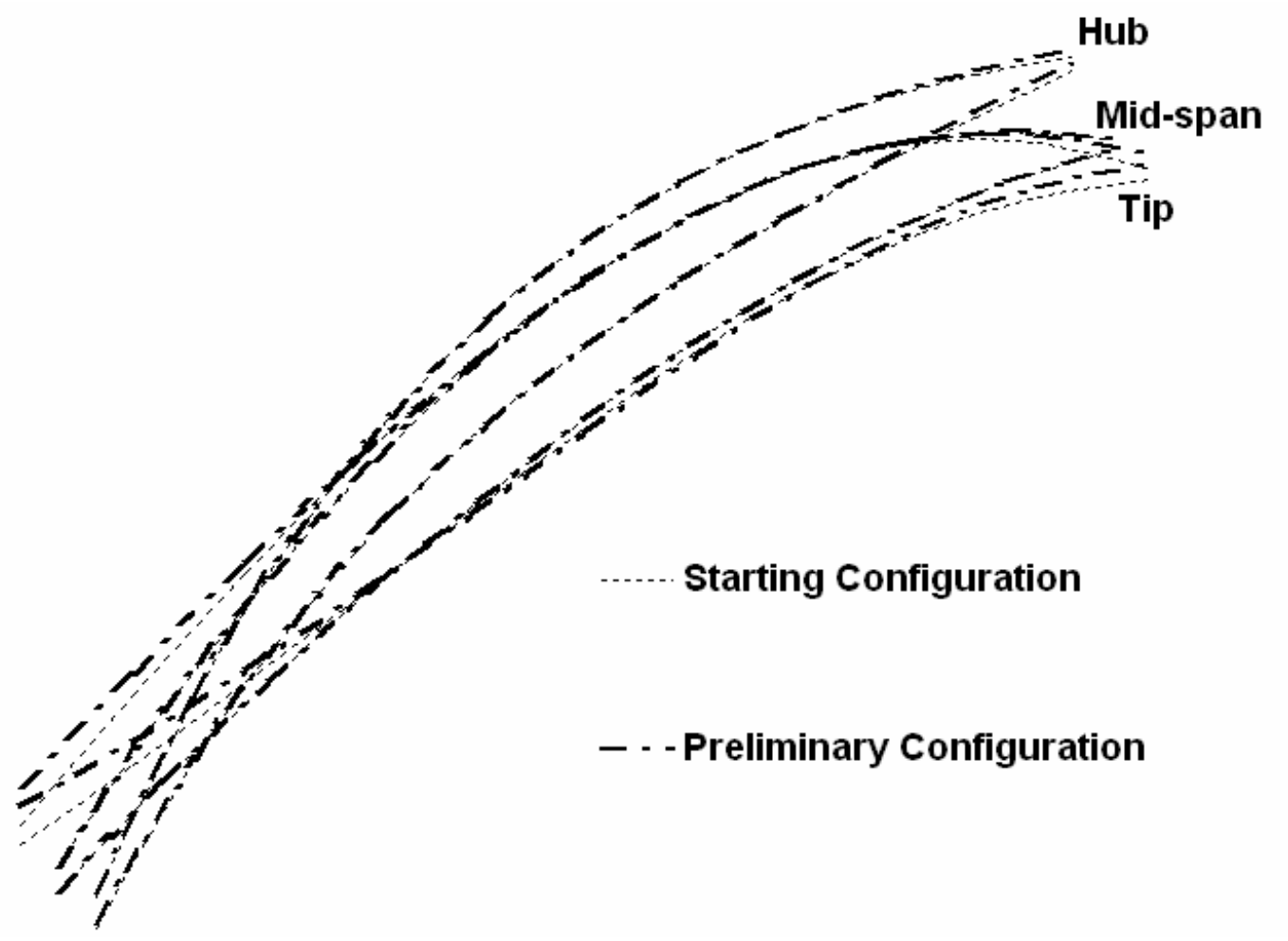

Figure 5.5: Stator blade profile comparisons between the starting and preliminary configurations at hub, mid-span and tip locations. 


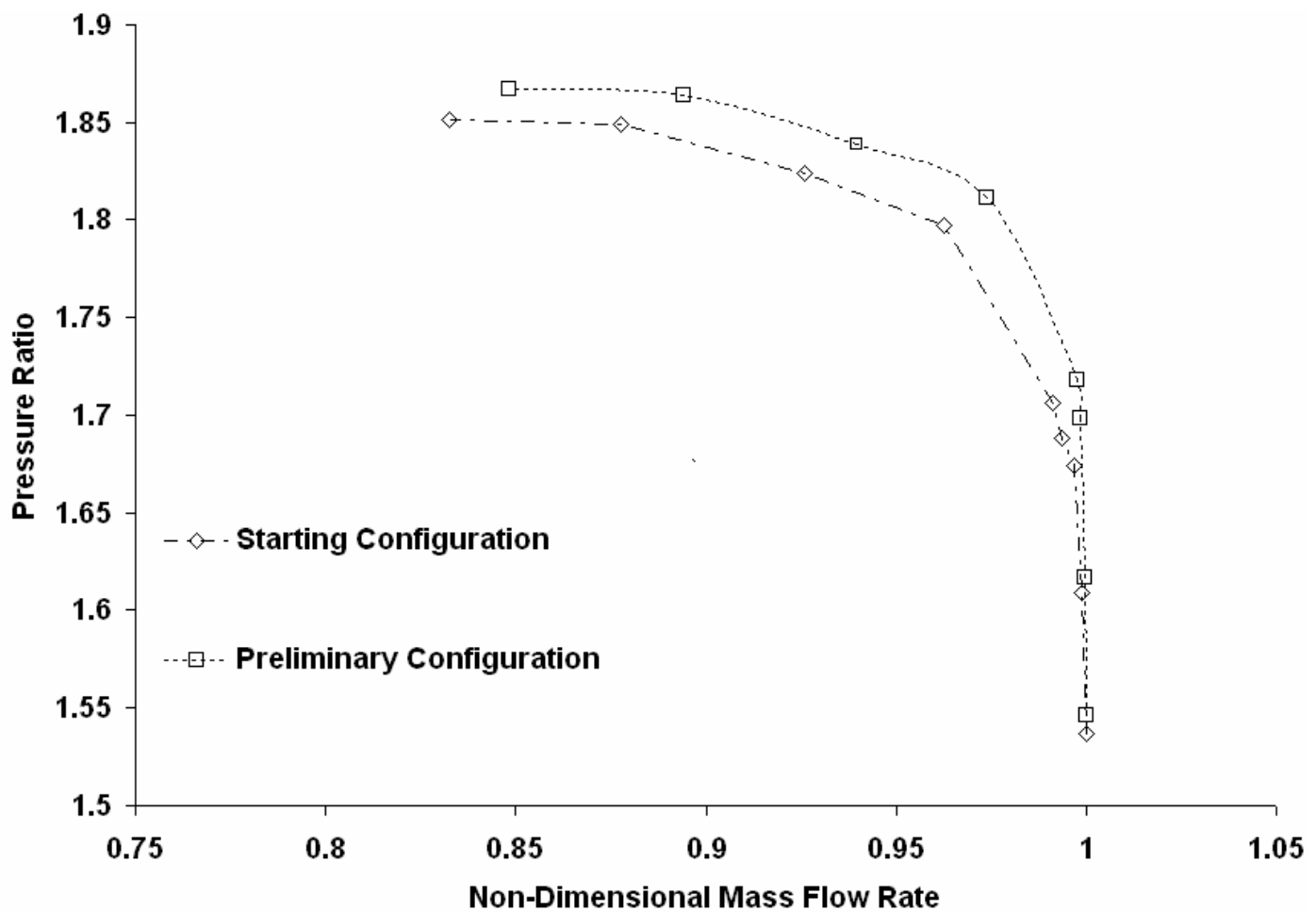

Figure 5.6: Performance map comparing the Starting configuration to the Preliminary configuration.

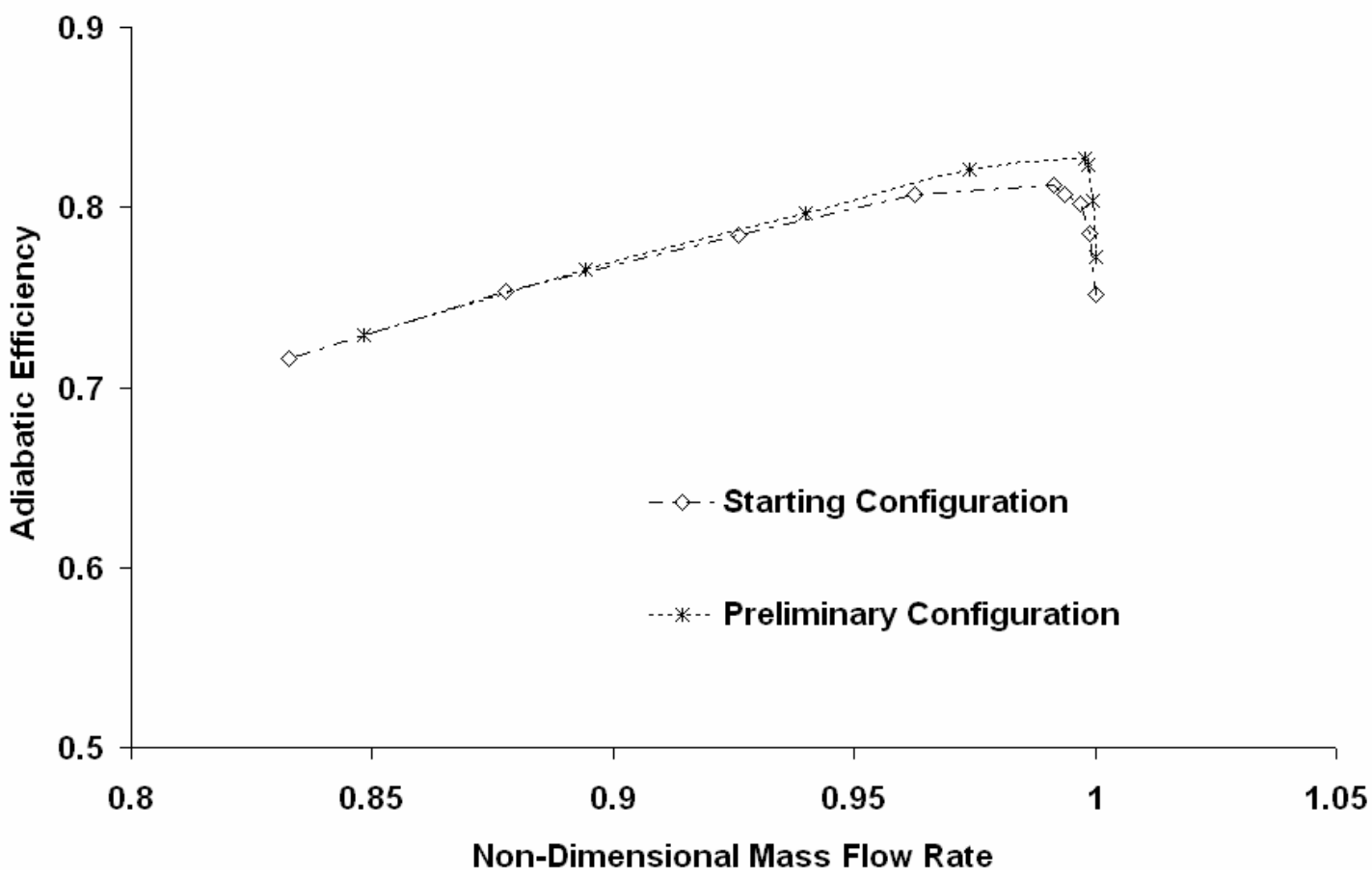

Figure 5.7: Adiabatic efficiency map comparing the Starting configuration to the Preliminary configuration. 


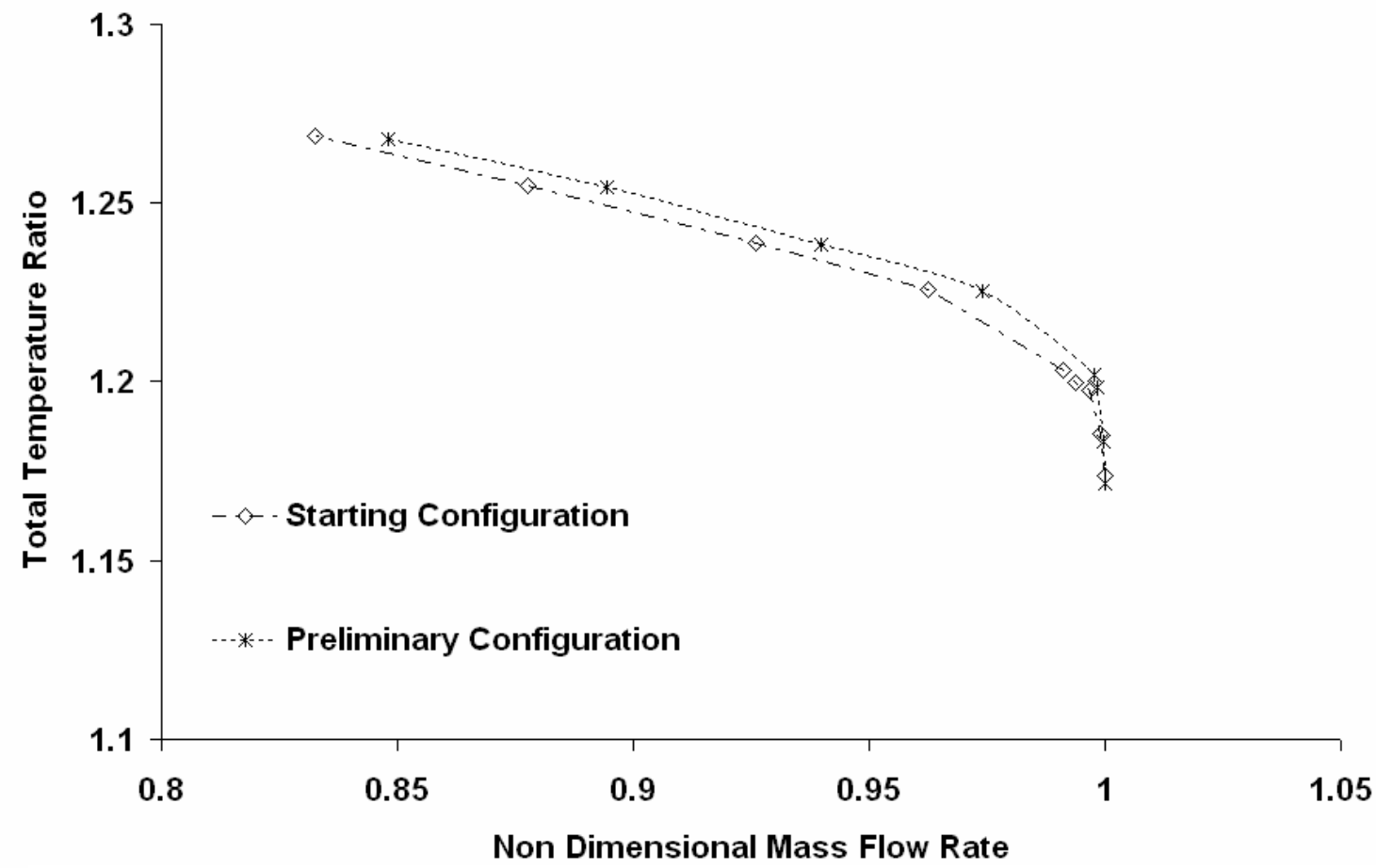

Figure 5.8: Total temperature ratio map comparing the Starting configuration to the Preliminary configuration.

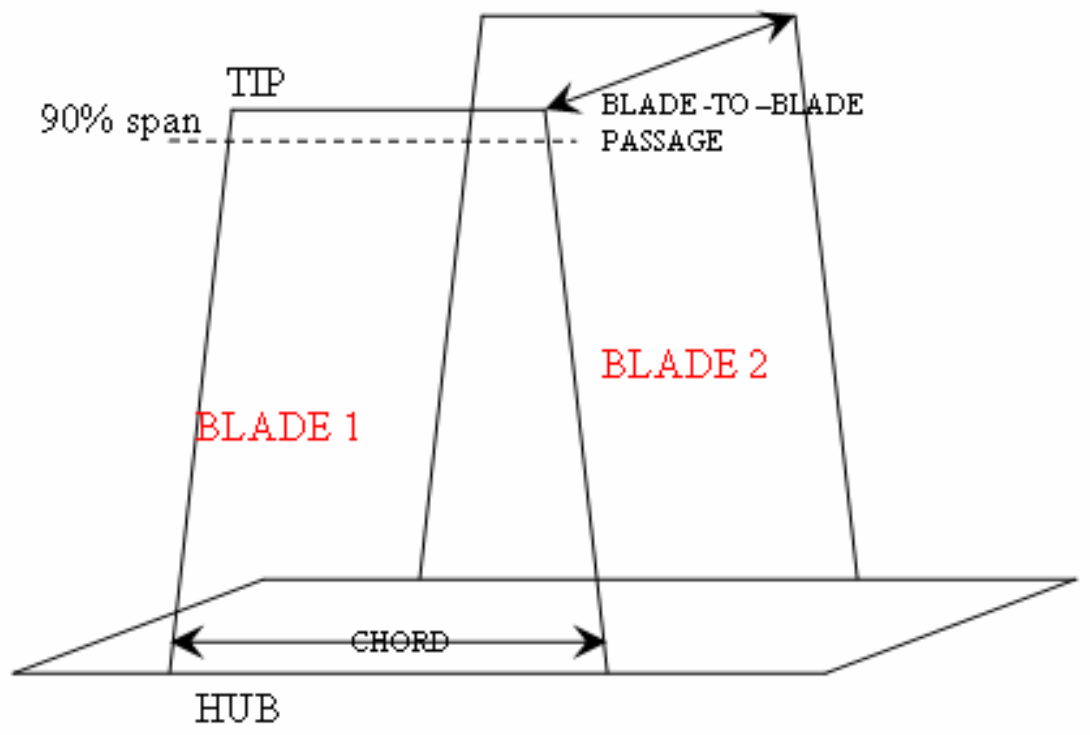

Figure 5.9: Schematic of the blade-to-blade passage for a typical axial compressor configuration. 

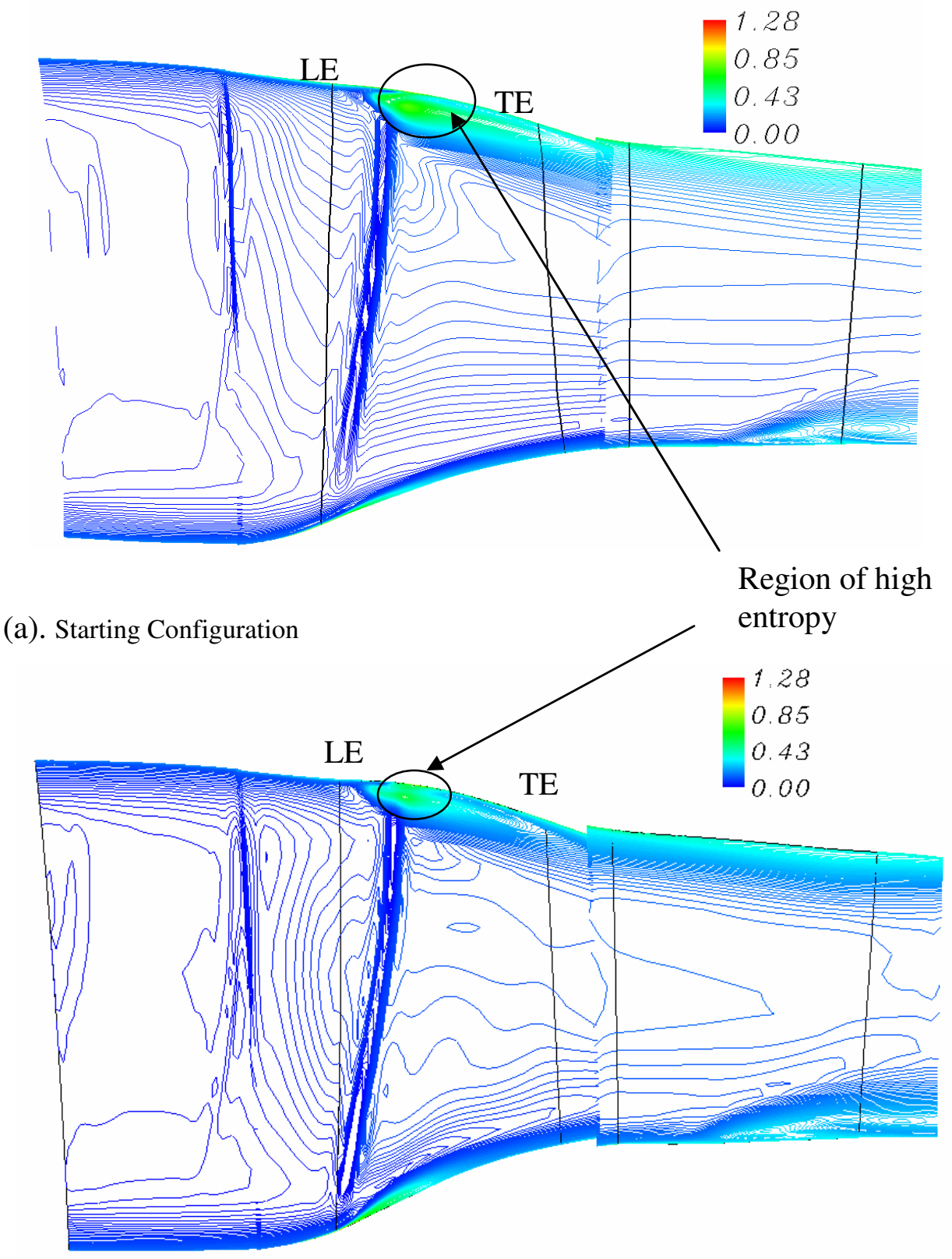

(b). Preliminary Configuration

Figure 5.10: Entropy (non-dimensional) contour comparison between the (a). Starting and (b). Preliminary configurations, for mid passage location at off-design condition ( $\operatorname{mdot}=0.87$ mdot_choke, $T_{\text {ref }}=288.3 \mathrm{~K}, \rho_{\text {ref }}=1.233 \mathrm{~kg} / \mathrm{m}^{3}$ ). 


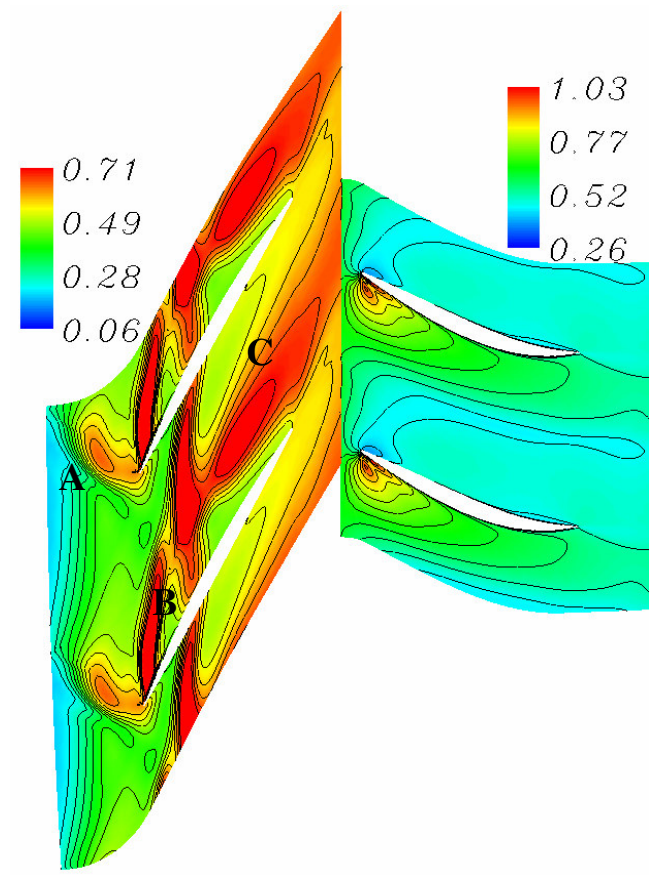

(a). Starting Configuration

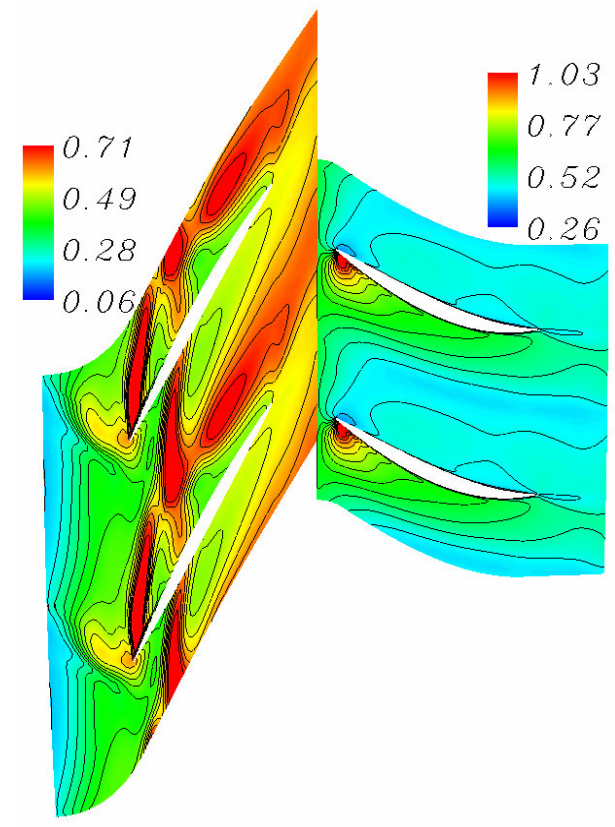

(b). Preliminary Configuration

Figure 5.11: Entropy (non-dimensional) contour comparison between the (a). Starting and (b). Preliminary configurations, for tip clearance section at off-design condition $(\mathbf{m d o t}=0.87$ mdot_choke, $\left.T_{\text {ref }}=288.3 \mathrm{~K}, \rho_{\text {ref }}=1.233 \mathrm{~kg} / \mathrm{m}^{3}\right)$. 


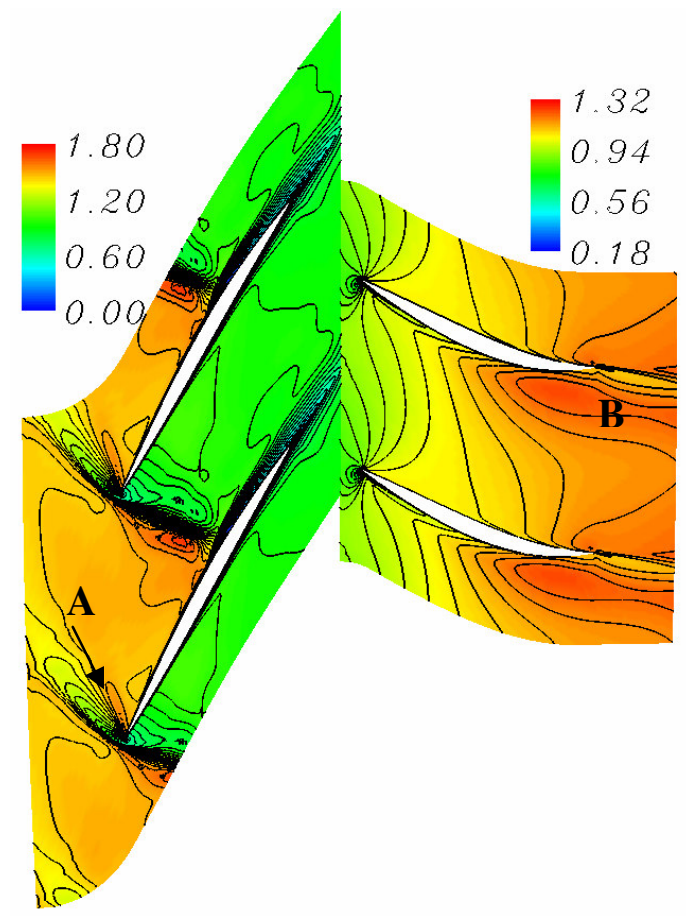

(a). Starting Configuration

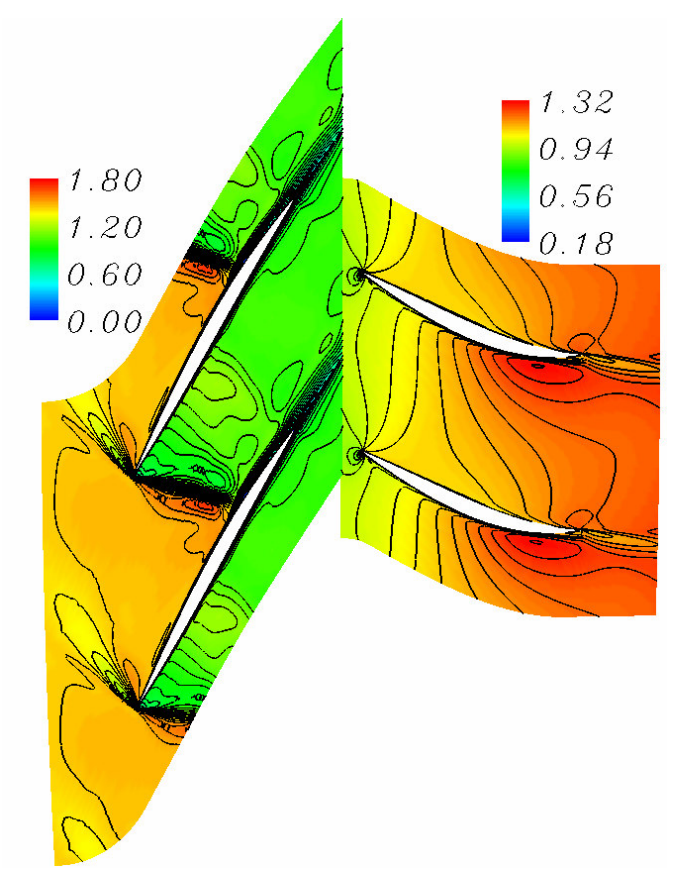

(b). Preliminary Configuration

Figure 5.12: Mach number contour comparison between the (a). Starting and (b). Preliminary configurations at $90 \%$ span from hub at design condition ( $m$ dot $=0.97$ mdot_choke). 


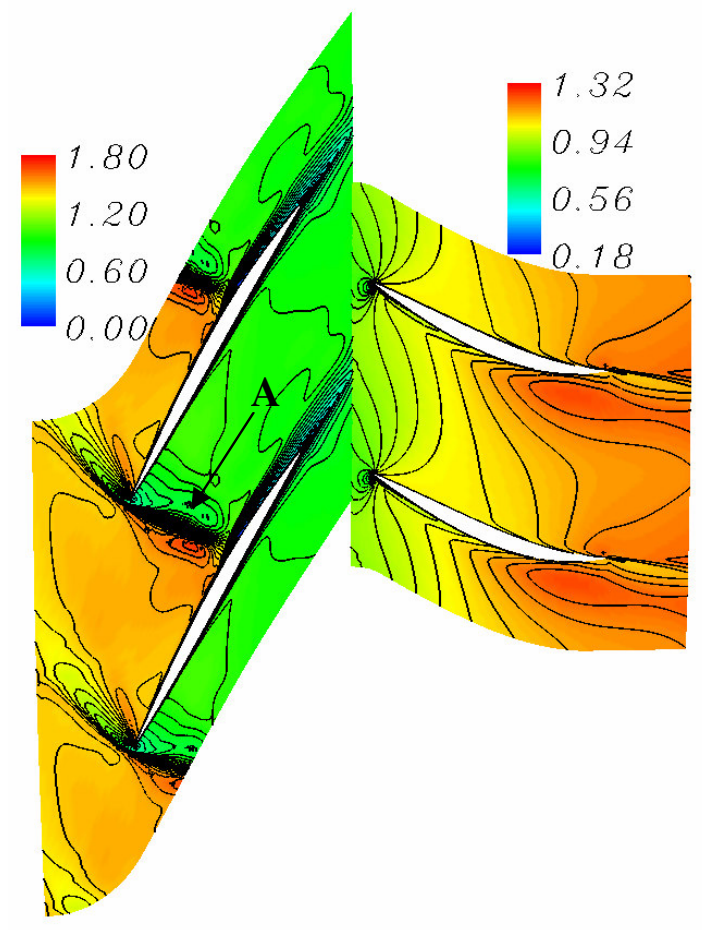

(a). Starting Configuration

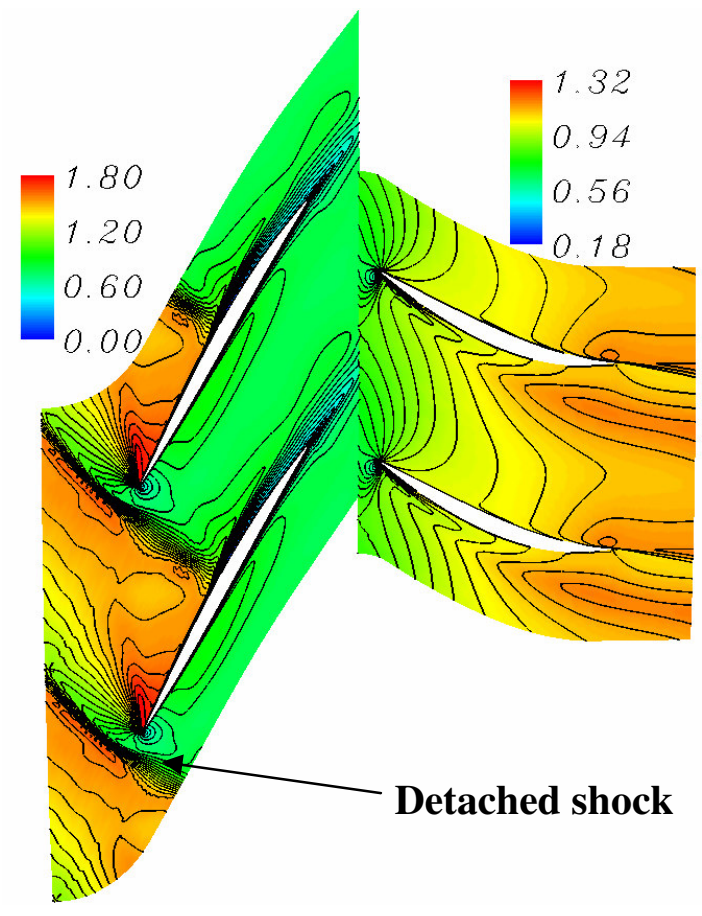

(b). Preliminary Configuration

Figure 5.13: Mach number contour comparison between the (a). Starting and (b). Preliminary configurations at $90 \%$ span from hub at off-design condition (mdot $=0.87$ mdot_choke). 


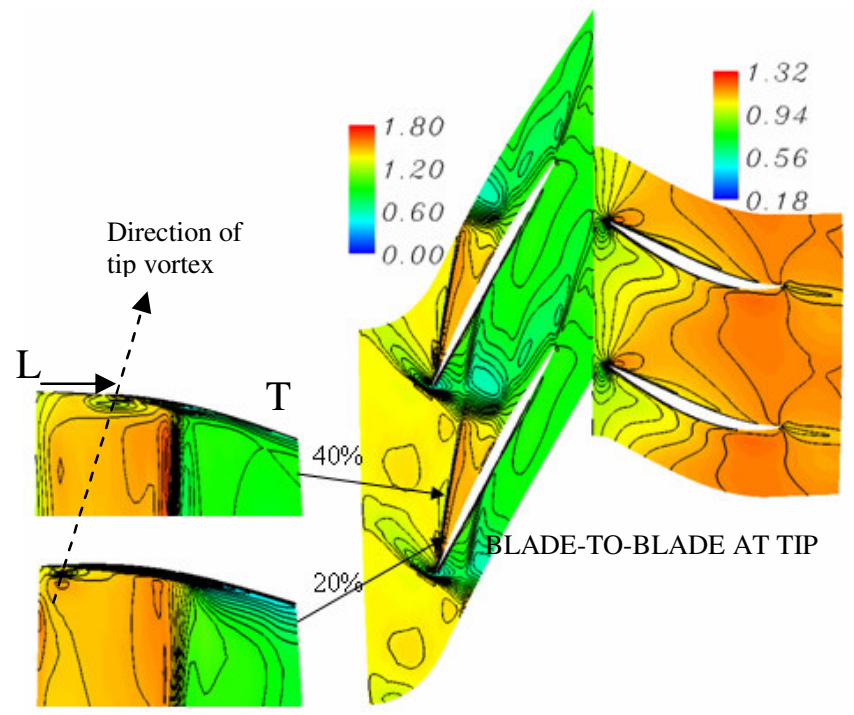

(a). Starting Configuration

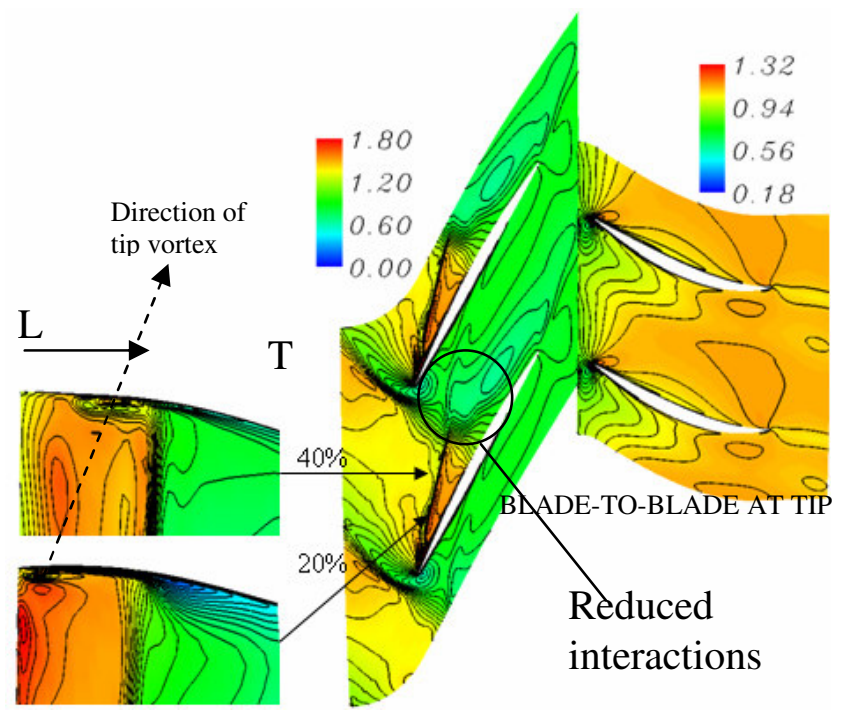

(b). Preliminary Configuration

Figure 5.14: Mach number contour comparison between the (a). Starting and (b). Preliminary configurations at tip off-design condition ( $\operatorname{mdot}=0.87 \mathrm{mdot}$ _choke) and two meridional planes above $70 \%$ span. 


\section{CHAPTER VI}

\section{RESULTS II: COMPREHENSIVE APPLICATION OF METHODOLOGY}

In the previous chapter results from the preliminary application of the methodology were presented. This chapter will present results obtained from the comprehensive application of the methodology. The proposed methodology is broadened such that more design variables are screened and are selected more intelligently. Also the objective functions in the extended methodology are multidisciplinary- aerodynamics and aeroacoustics.

A comprehensive design variables selection technique is used where the variability of each variable on the eventual objective function defines the measure of importance for the variables. Aeroacoustic and structural analysis are performed and the aeroacoustics is incorporated into the optimization loop along with the aerodynamic analyses.

Results from the design variables selection along with details of the optimization loop are presented. The newly optimized configuration is termed 'Optimized Configuration' for the entirety of this study. As previously done, results are presented first in form of performance maps. The visualization of the flowfield is used to interpret the results. Results from the aeroacoustic analysis are also presented to substantiate the aerodynamic findings. 


\subsection{Selection of Design Variables}

Eighteen variables were considered for screening, nine for the rotor and nine for the stator, these are shown in figure 6.1. The 18 variables result in a DoE with 39 experiments; a snapshot of the experiments was previously shown in table 3.1. In table 3.1, the case represent all variables set at their lowest setting, followed by 19 cases with each successive variable set at its highest setting while all other variables fixed at the lowest setting. A further set of 19 runs with each successive variable set at its lowest setting with all the variables fixed at the highest setting are made, followed by one run with all variables set at their highest settings, and one run with all variables at zero values.

The objective functions, which in this case were total pressure ratio, adiabatic efficiency and rotor wake influence coefficient (RWIC), are obtained from CFD analysis performed on the experiments. The Pareto curves for total pressure ratio, adiabatic efficiency and RWIC are shown in figures 6.2, 6.3 and 6.4 respectively. The alphabets A thru $\mathrm{R}$ represent the 18 variables that are described in figure 6.1 (Also see table 6.1). Each horizontal bar represents the effects of a single design variable on the response. From figure 6.2 it can be interpreted that variables F, D, H, A, G and E have approximately $90 \%$ contributions to the total pressure ratio. It is also seen that the variables $A, D, G, B, F$ and C contribute $90 \%$ of the variability in the adiabatic efficiency case as shown in figure 6.3. In the case of RWIC, as shown in figure 6.4, variables D, A, F, I, M, G, E, B and C contribute to approximately $90 \%$ of its variability. 
Clearly some of the significant variables are common to all the responses. A closer look at the variables suggests that the prominent variables are primarily associated with the rotor blade, as compared to the stator blade. This evidently means that, when compared to the stator, the design of the rotor has a greater impact on the compressor stage performance functions considered in this study.

This result leads to an interesting finding which was previously not realized. Earlier, equal numbers of rotor and stator design variables were chosen as design variables. Although it led to an preliminary configuration, the screening performed in this section suggests that a larger set of variables is necessary, especially from the rotor design. Ten variables were selected based on its variability as the decisive design variables for the design of the compressor stage. The variables, as tabulated in table 6.2, are split such that 8 variables are for the rotor and the other 2 are for the stator. The design of experiments obtained subsequently for these 10 variables are shown in table 6.3

\subsection{Optimization Results}

A multi-objective optimization was performed where the total pressure ratio, adiabatic efficiency and RWIC were equally weighted. The optimizer was executed with the intent of maximizing the stage adiabatic efficiency, stage total pressure ratio and minimizing the rotor wake influence coefficient across the rotor-stator interface. The optimum values for the ten design parameters selected are given in tables 6.4 (rotor) and 6.5 (stator). As mentioned previously, the blade section constructed using the optimum 
values is referred to as the 'optimized configuration'. Figure 6.5 for the rotor blade and figure 6.6 for the stator blade, show comparisons between the starting and optimized configuration at hub, mid-span and tip locations.

For the stator blade some differences between the starting and optimized configuration can be observed at all three spanwise locations. At the hub the trailing edge incidence angle for the optimized profile is much greater than that for the starting configuration. Also a larger amount of camber is evident at the hub for the optimized profile. At mid-span a smaller camber at the aft of the blade is present for the optimized configuration. A similar observation can be made for the optimized profile at the tip section. Another difference at the tip location is that the optimized profile has a much higher incidence angle.

In case of the rotor blade, significant differences can be noticed at the hub and tip leading edge where the optimized profile has a higher leading edge incidence angle. At the mid span trailing edge the optimized profile has a lower incidence angle. One cause of concern at the hub is that profile changes very rapidly making it a challenging to manufacture. Consequences of these stator and rotor profile differences on the stage performance are discussed later.

A comparison between the design values predicted by the response surface equations (RSEs) and computed value by the CFD analysis for the three objective functions are given in table 6.6. From the table is evident that the computed values are very close to the values predicted by the response surface equations, which is a desirable result. This further confirms that the RSEs obtained for the data fit well and that they give 
a true representation of the relationship between the design variables and the objection functions. Figure 6.7 shows the response surfaces obtained for the three objective functions- total pressure ratio, adiabatic efficiency and rotor wake influence coefficient as a function of the two design parameters $\mathrm{A}$ (x-axis) and $\mathrm{B}$ (y-axis). In reality such response surfaces exist for each combination of design parameters and are used to locate the maximum/minimum for each combination. The location of maximum and minimum are automated in $\mathrm{JMP}^{\odot}$.

\subsubsection{Assessment of Blade Loading}

In order to make a quick assessment of the blade performance, the change in blade loading is assessed. Figures 6.8 (rotor) and 6.9 (stator) show the blade loading comparison between the starting and optimized configurations at three spanwise locations (hub, mid span and tip). In the figures the non-dimensional static pressures are plotted

against the distance $(\bar{s})$ on the blade. Usually the area under such a loading curve is a measure of the amount of loading on the blade surface. It can be said that the higher the loading on the blade, the greater the loading on the blade. The increased loading corresponds to the blade doing more work on the flow, which can result in higher stage total pressure ratio.

From figure 6.8 it can be seen that the optimized rotor blade experiences a slight increase in blade loading at hub (figure 6.8a), mid span (figure 6.8b) and tip (figure 6.8c) when compared to the starting configuration rotor blade, especially at the rotor leading 
edge. From the plots it is noticed that the incidence of the optimized configuration has been changed. At the tip (figure 6.8c) it is seen that the optimized configuration has a stronger shock when compared to the starting configuration. Although the shock is stronger, no loss of overall stage efficiency (presented later) was observed. For the stator blade it is clear that the optimized configuration has an increased blade loading at mid span (figure 6.9b) and tip (figure 6.9c) when compared to the starting configuration. The blade incidence has also been significantly changed going from the starting configuration to the optimized configuration. The change in incidence can better align the blade to the flow and also alter the direction of the tip vortex. A detailed discussion on direction of tip vortex and its effects on blockages and stage performance will be presented later.

\subsubsection{Performance map comparisons between Starting and Optimized}

\section{Configurations}

Performance maps for the starting configuration and the optimized configuration were obtained. In the map, as shown in figure 6.10, again the total pressure ratio of the stage is plotted as function of the ratio of the mass flow rate to the choking mass flow rate of the respective configurations. For the optimized configuration, the choking point was found to be $20.76 \mathrm{~kg} / \mathrm{sec}$, compared to $19.8 \mathrm{~kg} / \mathrm{sec}$ for the starting configuration. On comparing the optimized configuration to the starting configuration, it can be noticed that the optimized case exhibits on average a $2.5 \%$ increase in total pressure ratio over the entire operating range. The operating range for the optimized configuration shows a slight improvement over the starting configuration. 
Figure 6.11 shows the adiabatic efficiency map for the two configurations. From the efficiency map it can be observed that the difference in peak adiabatic efficiency between the two configurations is significant, especially at peak-efficiency. Near choke conditions, the optimized configuration has $2.5 \%$ higher peak efficiency than the starting configuration. Some adiabatic efficiency benefits for the optimized configuration are also seen at lower mass flow rates. The higher peak efficiency close to design point exhibited by the optimized configuration makes it an appealing option over the starting configuration.

A plot comparing the rise in total temperature over the stage for the starting configuration with the optimized configuration is shown in figure 6.12 . It can be observed that the total temperature ratio for the optimized configuration shows a slight $(0.5 \%)$ increase when compared to the starting configuration.

Until now all the calculations have been performed at 100\% rotor RPM. An offdesign speed analysis was performed to assess the performance at a different rotor rotational speed- 70\% RPM. Figure 6.13 shows the performance map for starting configuration and optimized configurations both at 70\% RPM. It is seen that the optimized case exhibits on average a $2.00 \%$ increase in total pressure ratio over the entire operating range. 


\subsubsection{Rotor-Stator aeroacoustic interaction comparisons between Starting and Optimized Configurations}

In order to evaluate the rotor-stator interactions, the blade-to-blade pressure fluctuations at mid-span and mid passage are monitored. The pressure fluctuations caused by the rotor design provide a way to quantify the potential disturbances and noise that may propagate both upstream and downstream. If the disturbances travel downstream then it may interact with the stator blades, by changing the flow angle at the stator leading edge. On the other hand if the disturbance travels upstream from the rotor it may affect the oncoming flow onto itself.

Figure 6.14 shows a plot comparing the blade-to-blade pressure fluctuations at rotor exit (stator entrance) for both the starting and optimized configurations. The fluctuations are measured about a circumferentially averaged pressure value at midpassage. In the plot the y-axis shows the fluctuating pressures $\left(P^{\prime}\right)$ and the $x$-axis represents the blade-to-blade circumferential location where $y / y^{*}=0$ and $y / y^{*}=1$ are labeled. It can be observed from the figure that the pressure fluctuations aft of the rotor blade are less for the optimized configuration compared to the starting configuration at most of the circumferential location. It is seen that the magnitude of the pressure fluctuations in the blade wake region are smaller for the optimized configuration when compared to the starting configuration. In the region $y / y^{*}=0.6$ to $y / y^{*}=1.0$, it can be observed that the optimized configuration has smaller magnitude and smaller fluctuations when compared to starting configuration. 
In order to assess the rotor forward noise, the blade-to-blade pressure fluctuations at the entrance to the stage, upstream of the rotor, is plotted in figure 6.15 for the starting and optimized configurations. In the plot the y-axis shows the fluctuating pressures $\left(P^{\prime}\right)$ and the $\mathrm{x}$-axis represents the blade-to-blade circumferential location where $\mathrm{y} / \mathrm{y}^{*}=0$ and $y / y^{*}=1$ are labeled. It is evident from the figure that the fluctuations for the optimized configuration and the starting configuration are almost identical. The magnitude of pressure fluctuations is slightly higher for the optimized configuration. No aeroacoustic performance benefits are observed at the rotor inlet.

Figure 6.16 shows the pressure fluctuations in the radial direction, from the hub to the tip. Here the fluctuations are measured about a radially averaged pressure value. In the plot the y-axis shows the fluctuating pressures $\left(P^{\prime}\right)$ and the $\mathrm{x}$-axis represents the hubto-tip radial location where $\mathrm{z} / \mathrm{z}^{*}=0$ and $\mathrm{z} / \mathrm{z}^{*}=1$ are labeled. In the region $\mathrm{z} / \mathrm{z}^{*}=0.25$ to $\mathrm{z} / \mathrm{z}^{*}=0.9$, it can be observed that the optimized configuration has smaller magnitude and smaller fluctuations when compared to starting configuration. In the tip section $\left(\mathrm{z} / \mathrm{z}^{*}=\right.$ 0.975) the optimized configuration has slightly higher $P^{\prime}$ magnitude when compared to the starting configuration.

\subsubsection{Flowfield comparisons between Starting and Optimized Configurations}

The flow fields for the starting and optimized configurations are compared to quantify the differences observed in the performance maps. Flowfield comparisons have 
been done off-design condition $\left(0.87 \dot{m}_{\text {choke }}\right)$ 'OD' on figure 6.9. The design point ' $\mathrm{D}$ ' $\left(0.97 m_{\text {choke }}\right)$ is also shown on figure 6.10 . In figure 6.17 the directions of the rotor leading edge tip vortex for the starting configuration and the optimized configuration are compared. The strengths of the tip vortex are also compared by superimposing the entropy on the particle path. Two notable differences can be observed. Firstly, the direction and width of the tip vortex for the optimized configuration has been significantly altered as it is seen that the tip vortex moves away from the neighboring blade and is narrower. Secondly, it is observed that the tip vortex entropy strengths are much weaker for the optimized configuration when compared to the starting configuration. The reduced entropy strength along with the altered tip vortex direction results in reduced blockages in the mid-passage section and can have significant aerodynamic performance benefits. The altered tip vortex direction can be correlated to the changed rotor blade incidences observed in section 6.2.1.

Figure 6.18 compares the entropy contours at mid-passage in the meridional plane for the starting and optimized configurations at the off-design condition. For the starting configuration a region of a significant region of high entropy exists in the tip section near the leading edge. This high entropy is attributed to the tip clearance vortex discussed previously. It can be noticed that the casing boundary layer is separated near the rotor leading edge because of the shock and the clearance vortex interactions. In case of the optimized configuration a much less pronounced region of high entropy can be observed at the rotor tip, suggesting that the strength of shock-clearance vortex interaction is lesser for the optimized configuration. Similar result was also observed in figure 6.17. 
Figure 6.19 compares the entropy contours for an off-design condition $\left(0.87 m_{\text {choke }}\right)$ for the starting configurations and optimized at tip clearance section in the blade-to-blade plane. As done previously, the results have been periodically rotated to reflect adjacent blade passages. On figure 6.19a, three areas are marked as A- ahead of the blade, B- near the rotor leading edge above the blade and $\mathrm{C}$ - mid passage wake region. At point $\mathrm{A}$, the region of high entropy previously present for the starting configuration is now non-existent for the optimized case. At the leading edge (Point B) a very rapid change of entropy is noticed, this is caused by the shock which is relatively weaker for the optimized case compared to the starting. In the mid passage wake region (Point C), the blade-to-blade wake entropy strength for the optimized configuration is significantly lower than that for the starting configuration.

Overall, this indicates that the blockages both upstream and downstream of the rotor blade are reduced. This has a substantial influence on the performance of the rotor especially at off-design conditions such as the one shown. The improvement in the wake region also effects the rotor wake-stator noise interactions and substantiates the reduced levels of aero-acoustic interactions found earlier in Section 6.2.3 for the optimized configuration.

Mach number contours comparing the starting and optimized configuration at $90 \%$ span location from the hub for off-design condition $\left(0.87 \dot{m}_{\text {choke }}\right)$ is shown on figure 6.20. On figure 6.20a smeared shock emerging from the lower surface (labeled A) at the rotor leading edge can observed for the starting configuration. This shock passes through the entire passage eventually ending at the upper surface of the next blade. In the process 
the shock interacts with the boundary layer on the upper surface of the next blade and can at times trigger boundary layer separation. From figure $6.20 \mathrm{~b}$ it is noticed that the shock emanating from the rotor lower surface does not exist for the optimized configuration. Instead a weaker detached shock that loses its strength before it can reach the neighboring rotor blade is observed, and the detached shock does not affect the flow on the upper surface of the next rotor blade.

From figure 6.5 it can be observed that the rotor has higher incidence angles at the leading edge. This causes the oncoming flow to align itself much better than before, reducing any interactions than might have been existent before in the vicinity of the leading edge. In figure 6.6 the stators' profile for the optimized configuration has a smaller camber than the starting case, especially at the trailing edge. As a result of the reduced stator camber the optimized profile experiences a smaller stator loading. This is evident from of the lower mach numbers noticed in the vicinity of region B in figure $6.20 \mathrm{~b}$.

Figure 6.21 shows the effects of the shock and clearance flow on the casing boundary layer. Blade-to-blade plot of Mach number contours for starting (Figure 6.21a) and optimized configuration (Figure 6.21b) at the blade tip- off design condition are shown at the right of the figure. This close to the casing the shock is highly smeared, and the tip vortex can be followed through the shock to the pressure side of the neighboring blade. Two meridional plots of Mach number contours above 70 percent span at two tangential locations (20\% and 40\%) are shown on the left. The leading edge and trailing edges are also shown for reference. 
In figure 6.21a, the bottom left plot is near the suction surface of the blade shows the clearance vortex just downstream of the leading edge, followed by a region of lowspeed flow. The shock is evident at near $50 \%$ chord, followed by an even larger region of low-speed flow caused by the shock-boundary layer interaction. At $40 \%$ tangential location the clearance vortex is evident at approximately $30 \%$ chord. The dotted arrow shows the propagation of the vortex in the azimuthal direction. The solid arrow indicates the chordwise distance of the clearance vortex from the leading edge.

For the optimized configuration, it is observed from figure $6.21 \mathrm{~b}$ that the azimuthal convection of the clearance vortex is slightly different. The tip vortex for the optimized configuration appears to be traveling at higher angle relative to the normal when compared to figure $6.21 \mathrm{~b}$. Also the chordwise position of the same clearance vortex is at a greater chordwise location $(\sim 45 \%)$, which is again indicated by the longer solid arrow with leading edge as the reference. By altering the propagation direction of the tip clearance vortex for the optimized configuration, any blockage effects due to the interaction between the tip vortex and blade-to-blade shocks are reduced.

In the blade-to-blade plane on the right side of figure 6.21a, a strong shock emerging from the suction side of blade leading edge is evident for the starting configuration. Comparing this shock to its counterpart in the optimized configuration in figure $6.21 \mathrm{~b}$, it is seen that this shock for the latter is not as strong as the one for the starting configuration. The shock for the optimized configuration is also not as smeared as compared to the starting configuration and does not extend through the entire passage. It is noticed again that the interactions between the shock emerging from pressure side 
and the neighboring blade are very minimal for the optimized configuration compared to the starting one.

Once again it is found the aerodynamic changes have a beneficial effect on the performance of the compressor stage. It can be construed that the blockage modifications and reduced shock strengths result in increased total pressure ratios across the stage for no additional penalty in the form of decrease in adiabatic efficiency.

\subsection{Structural Analysis on Optimized Configuration}

Modern axial compressor blades are, by design, thin and heavily loaded. This leads to large deflections and changes in the spanwise distribution of the blade geometry. These deflections could lead to large changes in the performance. Although it is not possible to measure these deflections in the operating conditions, it is important to account for these deflections in numerical calculations. In this study the aerodynamic loads obtained from CFD are coupled in an open loop fashion with a structural analysis software ANSYS to account for the blade deflections under normal operating conditions. The intent is to demonstrate a method by which blade deflections maybe calculated and accounted for. A full non-linear blade structural analysis incorporating the blade failure criteria is beyond the scope of this study. Results obtained from a structural analysis on the optimized configuration are presented.

The blade shapes used until now have the aerodynamic forces already accounted for, and are referred to as the 'hot shape'. The oncoming flow essentially sees the 'hot 
shape' of the blade and not the manufactured blade shape. Hence a modified shape, obtained by subtracting the deflections due to aerodynamic loading from 'hot shape', also referred to as 'cold shape', is obtained. Figure 6.22 shows the hot and cold shapes for the rotor blades.

Figure 6.23 shows the displacement on the pressure side (figure 6.23a) and suction side (figure 6.23b) of the rotor blade. In figure $6.23 \mathrm{a}$ it can be seen that the maximum displacement on the pressure surface occurs at mid-chord near the tip. In case of the suction surface, it is seen from the figure $6.23 \mathrm{~b}$, that the maximum deflections occur at $75 \%$ chord in the tip section. In both cases it is observed that the least deflections occur at the hub, which is expected as the blade is rooted to the hub. To better understand the von Mises stress distribution on the new blade, the stresses on both the pressure and suction surfaces are show on figures $6.24 \mathrm{a}$ and figure $6.24 \mathrm{~b}$ respectively. At the operational condition, the deformation and von Mises stress in are largely caused by the centrifugal loads rather than caused by the aerodynamic pressure force. It is seen that the stresses are maximum at the hub, which may be expected as modern compressor blades are subject to high amount of hub stresses.

Similar displacement analysis was performed on the stator blade. The deflections on the stator blade, not shown here, were found to be very small. It should be noted that the stator blade is rooted both at hub and tip. In the absence of centrifugal forces, the deformation and von Mises stress in are largely caused by the aerodynamic pressure forces. The von Mises stress distribution on the suction surface is shown in figure 6.25 . It 
is seen that maximum stresses occur at the hub and the casing where the stator blade is rooted.

\subsection{Reanalysis on Baseline Stage 35 configuration}

From the design methodology an optimized configuration was obtained. The optimized configuration exhibits better aerodynamic and aeroacoustic characteristics than the starting configuration. A need still exists to apply differences obtained from the design methodology to the baseline Stage 35 configuration. In this section the differences (deltas) observed between the starting and optimized configurations are applied to the baseline Stage 35 configuration. First, the changes in the blade configuration for the starting and optimized configuration are obtained. Then these shape changes are added to the baseline Stage 35 configuration to obtain the Stage 35-2 configuration. This Stage 352 configuration is reanalyzed to obtain the performance map.

Figure 6.26 compares the performance map obtained computationally for the baseline Stage 35 and the Stage35-2 configurations. From the map it is clear that the Stage35-2 configuration shows very comparable results to the baseline Stage 35 configuration. A slight extension (1\%) in operating range is observed for the Stage 35-2 configuration. The adiabatic efficiency map for the baseline Stage 35 and the Stage35-2 configurations is shown on figure 6.27. From the efficiency map it is seen that the two configurations have comparable adiabatic efficiencies but the Stage 35-2 configuration exhibits higher efficiencies at lower mass flow rates. 
The benefits noticed from the optimized configuration previously discussed were not observed in this case. It is because the starting configuration had a misaligned flow incidence due to the parameterization errors and the optimized configuration had the incidence better aligned with the incoming flow (discussed in section 6.2.1). Here the baseline configuration- Stage35 does not have the misaligned flow incidence seen previously. Figure 6.28 shows the rotor blade loading comparisons between the Stage 35 configuration and the Stage 35-2 configuration at mid-span. From the plot it is clear that blade loading has not changed much between the two configurations. The changes in blade incidences observed previously are also not seen here. Figure 6.29 shows the stator blade loading comparisons between the Stage 35 configuration and the Stage 35-2 configuration at mid-span. The blade loading for the two stator configurations are similar and are not notably different. 


\begin{tabular}{|l|l|}
\hline & \multicolumn{1}{|c|}{ VARIABLE } \\
\hline A & Rotor leading edge camber angle- hub \\
\hline B & Rotor mid-chord camber angle- hub \\
\hline C & Rotor trailing edge camber angle- hub \\
\hline D & Rotor leading edge camber angle- mid-span \\
\hline E & Rotor mid-chord camber angle- mid-span \\
\hline F & Rotor trailing edge camber angle- mid-span \\
\hline G & Rotor leading edge camber angle- tip \\
\hline H & Rotor mid-chord camber angle- tip \\
\hline I & Rotor trailing edge camber angle- tip \\
\hline J & Stator leading edge camber angle- hub \\
\hline K & Stator mid-chord camber angle- hub \\
\hline L & Stator trailing edge camber angle- hub \\
\hline M & Stator leading edge camber angle- mid-span \\
\hline N & Stator mid-chord camber angle- mid-span \\
\hline O & Stator trailing edge camber angle- mid-span \\
\hline P & Stator leading edge camber angle- tip \\
\hline Q & Stator mid-chord camber angle- tip \\
\hline Stator trailing edge camber angle- tip \\
\hline
\end{tabular}

Table 6.1: Summary of the 18 design variables considered for the screening study. 


\begin{tabular}{|c|l|}
\hline & VARIABLE \\
\hline A & Rotor leading edge camber angle- hub \\
\hline B & Rotor mid-chord camber angle- hub \\
\hline D & Rotor leading edge camber angle- mid-span \\
\hline E & Rotor mid-chord camber angle- mid-span \\
\hline F & Rotor trailing edge camber angle- mid-span \\
\hline G & Rotor leading edge camber angle- tip \\
\hline H & Rotor mid-chord camber angle- tip \\
\hline I & Rotor trailing edge camber angle- tip \\
\hline M & Stator leading edge camber angle- mid-span \\
\hline O & Stator trailing edge camber angle- mid-span \\
\hline
\end{tabular}

Table 6.2: Final design variables selected from screening studies. 


\begin{tabular}{|c|c|c|c|c|c|c|c|c|c|c|}
\hline & $A$ & $B$ & D & $E$ & $F$ & $\mathrm{G}$ & $\mathrm{H}$ & 1 & $M$ & 0 \\
\hline 1 & 47.2 & 18.4 & 52.2 & 50.4 & 24.8 & 68.6 & 54.4 & 50.2 & 43.1 & -1 \\
\hline 2 & 49.2 & 17.4 & 54.2 & 47.4 & 18.8 & 68.6 & 54.4 & 42.2 & 39.1 & -1 \\
\hline 3 & 41.2 & 17.4 & 54.2 & 46.4 & 20.8 & 60.6 & 53.4 & 44.2 & 39.1 & -3 \\
\hline 4 & 45.2 & 18.4 & 46.2 & 49.4 & 20.8 & 68.6 & 51.4 & 46.2 & 41.1 & -9 \\
\hline 5 & 47.2 & 21.4 & 48.2 & 46.4 & 26.8 & 64.6 & 50.4 & 44.2 & 43.1 & -9 \\
\hline 6 & 49.2 & 19.4 & 48.2 & 50.4 & 22.8 & 60.6 & 51.4 & 50.2 & 35.1 & -9 \\
\hline 7 & 41.2 & 21.4 & 46.2 & 46.4 & 26.8 & 60.6 & 50.4 & 50.2 & 39.1 & -3 \\
\hline 8 & 47.2 & 18.4 & 52.2 & 46.4 & 24.8 & 66.6 & 51.4 & 44.2 & 35.1 & -1 \\
\hline 9 & 43.2 & 18.4 & 46.2 & 47.4 & 22.8 & 66.6 & 53.4 & 48.2 & 41.1 & -1 \\
\hline 10 & 43.2 & 20.4 & 52.2 & 50.4 & 24.8 & 62.6 & 54.4 & 44.2 & 37.1 & -1 \\
\hline 11 & 47.2 & 17.4 & 46.2 & 49.4 & 18.8 & 62.6 & 53.4 & 46.2 & 43.1 & -3 \\
\hline 12 & 49.2 & 20.4 & 50.2 & 50.4 & 18.8 & 68.6 & 53.4 & 50.2 & 35.1 & -5 \\
\hline 13 & 43.2 & 17.4 & 46.2 & 46.4 & 18.8 & 66.6 & 50.4 & 42.2 & 43.1 & -3 \\
\hline 14 & 43.2 & 17.4 & 52.2 & 50.4 & 26.8 & 66.6 & 53.4 & 42.2 & 43.1 & -9 \\
\hline 15 & 43.2 & 18.4 & 52.2 & 47.4 & 18.8 & 68.6 & 51.4 & 50.2 & 37.1 & -3 \\
\hline 16 & 43.2 & 18.4 & 52.2 & 50.4 & 18.8 & 62.6 & 54.4 & 50.2 & 41.1 & -5 \\
\hline 17 & 41.2 & 19.4 & 54.2 & 49.4 & 20.8 & 66.6 & 54.4 & 48.2 & 35.1 & -5 \\
\hline 18 & 47.2 & 20.4 & 46.2 & 46.4 & 24.8 & 60.6 & 53.4 & 42.2 & 39.1 & -3 \\
\hline 19 & 49.2 & 17.4 & 54.2 & 46.4 & 26.8 & 62.6 & 50.4 & 42.2 & 37.1 & -9 \\
\hline 20 & 49.2 & 21.4 & 48.2 & 47.4 & 24.8 & 60.6 & 53.4 & 48.2 & 35.1 & -5 \\
\hline 21 & 47.2 & 20.4 & 48.2 & 47.4 & 20.8 & 66.6 & 51.4 & 46.2 & 37.1 & -5 \\
\hline 22 & 49.2 & 21.4 & 54.2 & 49.4 & 26.8 & 68.6 & 50.4 & 48.2 & 37.1 & -1 \\
\hline 23 & 41.2 & 17.4 & 46.2 & 50.4 & 18.8 & 66.6 & 50.4 & 50.2 & 35.1 & -3 \\
\hline 24 & 45.2 & 20.4 & 54.2 & 49.4 & 24.8 & 60.6 & 54.4 & 44.2 & 43.1 & -7 \\
\hline 25 & 43.2 & 17.4 & 46.2 & 47.4 & 26.8 & 60.6 & 51.4 & 42.2 & 35.1 & -1 \\
\hline 26 & 43.2 & 20.4 & 48.2 & 46.4 & 18.8 & 62.6 & 50.4 & 48.2 & 41.1 & -7 \\
\hline 27 & 43.2 & 17.4 & 48.2 & 46.4 & 24.8 & 68.6 & 54.4 & 42.2 & 35.1 & -9 \\
\hline 28 & 45.2 & 18.4 & 48.2 & 49.4 & 24.8 & 64.6 & 53.4 & 50.2 & 37.1 & -3 \\
\hline 29 & 47.2 & 18.4 & 50.2 & 47.4 & 26.8 & 66.6 & 54.4 & 42.2 & 41.1 & -5 \\
\hline 30 & 49.2 & 17.4 & 46.2 & 50.4 & 24.8 & 66.6 & 51.4 & 42.2 & 39.1 & -5 \\
\hline 31 & 49.2 & 21.4 & 46.2 & 49.4 & 18.8 & 62.6 & 50.4 & 50.2 & 41.1 & -3 \\
\hline 32 & 41.2 & 21.4 & 52.2 & 49.4 & 20.8 & 62.6 & 52.4 & 44.2 & 39.1 & -9 \\
\hline 33 & 45.2 & 20.4 & 52.2 & 50.4 & 20.8 & 66.6 & 50.4 & 44.2 & 43.1 & -3 \\
\hline 34 & 41.2 & 20.4 & 54.2 & 50.4 & 18.8 & 60.6 & 52.4 & 50.2 & 35.1 & -1 \\
\hline 35 & 49.2 & 17.4 & 54.2 & 49.4 & 20.8 & 62.6 & 51.4 & 48.2 & 35.1 & -3 \\
\hline 36 & 43.2 & 21.4 & 46.2 & 46.4 & 18.8 & 62.6 & 54.4 & 44.2 & 35.1 & -3 \\
\hline 37 & 41.2 & 20.4 & 50.2 & 49.4 & 18.8 & 66.6 & 51.4 & 42.2 & 39.1 & -1 \\
\hline 38 & 41.2 & 21.4 & 46.2 & 50.4 & 18.8 & 60.6 & 51.4 & 42.2 & 43.1 & -7 \\
\hline 39 & 49.2 & 20.4 & 46.2 & 47.4 & 26.8 & 68.6 & 54.4 & 42.2 & 35.1 & -1 \\
\hline 40 & 47.2 & 21.4 & 52.2 & 46.4 & 20.8 & 62.6 & 53.4 & 48.2 & 43.1 & -3 \\
\hline 41 & 43.2 & 21.4 & 46.2 & 46.4 & 22.8 & 68.6 & 50.4 & 50.2 & 37.1 & -9 \\
\hline 42 & 49.2 & 20.4 & 48.2 & 49.4 & 20.8 & 64.6 & 54.4 & 42.2 & 41.1 & -1 \\
\hline
\end{tabular}




\begin{tabular}{|c|c|c|c|c|c|c|c|c|c|c|}
\hline & $A$ & $B$ & $D$ & $E$ & $F$ & $\mathrm{G}$ & $\mathrm{H}$ & I & $\mathrm{M}$ & 0 \\
\hline 43 & 47.2 & 20.4 & 54.2 & 47.4 & 24.8 & 62.6 & 50.4 & 50.2 & 41.1 & -7 \\
\hline 44 & 43.2 & 20.4 & 54.2 & 46.4 & 26.8 & 66.6 & 53.4 & 50.2 & 41.1 & -3 \\
\hline 45 & 47.2 & 20.4 & 54.2 & 46.4 & 20.8 & 62.6 & 54.4 & 42.2 & 35.1 & -9 \\
\hline 46 & 41.2 & 17.4 & 46.2 & 46.4 & 20.8 & 68.6 & 54.4 & 50.2 & 43.1 & -7 \\
\hline 47 & 41.2 & 19.4 & 52.2 & 47.4 & 26.8 & 62.6 & 53.4 & 48.2 & 37.1 & -7 \\
\hline 48 & 47.2 & 17.4 & 52.2 & 47.4 & 18.8 & 60.6 & 51.4 & 42.2 & 41.1 & -9 \\
\hline 49 & 49.2 & 18.4 & 46.2 & 46.4 & 18.8 & 60.6 & 54.4 & 50.2 & 37.1 & -1 \\
\hline 50 & 47.2 & 21.4 & 48.2 & 50.4 & 22.8 & 68.6 & 50.4 & 42.2 & 35.1 & -9 \\
\hline 51 & 49.2 & 21.4 & 54.2 & 46.4 & 18.8 & 60.6 & 50.4 & 44.2 & 35.1 & -9 \\
\hline 52 & 45.2 & 18.4 & 48.2 & 50.4 & 20.8 & 60.6 & 50.4 & 44.2 & 39.1 & -1 \\
\hline 53 & 49.2 & 19.4 & 48.2 & 46.4 & 24.8 & 68.6 & 50.4 & 48.2 & 43.1 & -3 \\
\hline 54 & 41.2 & 21.4 & 46.2 & 47.4 & 18.8 & 66.6 & 54.4 & 44.2 & 43.1 & -9 \\
\hline 55 & 43.2 & 21.4 & 54.2 & 47.4 & 24.8 & 60.6 & 50.4 & 42.2 & 37.1 & -3 \\
\hline 56 & 49.2 & 19.4 & 52.2 & 50.4 & 26.8 & 60.6 & 53.4 & 48.2 & 43.1 & -3 \\
\hline 57 & 45.2 & 19.4 & 50.2 & 48.4 & 22.8 & 64.6 & 52.4 & 46.2 & 39.1 & -5 \\
\hline 58 & 41.2 & 17.4 & 50.2 & 48.4 & 26.8 & 68.6 & 50.4 & 44.2 & 37.1 & -3 \\
\hline 59 & 43.2 & 21.4 & 46.2 & 47.4 & 22.8 & 60.6 & 54.4 & 50.2 & 43.1 & -9 \\
\hline 60 & 47.2 & 18.4 & 46.2 & 49.4 & 20.8 & 60.6 & 53.4 & 42.2 & 35.1 & -7 \\
\hline 61 & 47.2 & 21.4 & 54.2 & 50.4 & 20.8 & 68.6 & 53.4 & 42.2 & 39.1 & -1 \\
\hline 62 & 41.2 & 21.4 & 54.2 & 46.4 & 20.8 & 68.6 & 50.4 & 42.2 & 41.1 & -7 \\
\hline 63 & 47.2 & 17.4 & 54.2 & 46.4 & 18.8 & 64.6 & 53.4 & 50.2 & 35.1 & -9 \\
\hline 64 & 47.2 & 21.4 & 46.2 & 50.4 & 24.8 & 64.6 & 54.4 & 48.2 & 39.1 & -9 \\
\hline 65 & 49.2 & 18.4 & 46.2 & 46.4 & 20.8 & 62.6 & 53.4 & 44.2 & 43.1 & -9 \\
\hline 66 & 43.2 & 17.4 & 52.2 & 49.4 & 26.8 & 60.6 & 50.4 & 48.2 & 39.1 & -7 \\
\hline 67 & 43.2 & 18.4 & 54.2 & 50.4 & 18.8 & 64.6 & 51.4 & 42.2 & 37.1 & -7 \\
\hline 68 & 41.2 & 21.4 & 48.2 & 50.4 & 24.8 & 68.6 & 51.4 & 50.2 & 43.1 & -5 \\
\hline 69 & 45.2 & 17.4 & 54.2 & 47.4 & 26.8 & 62.6 & 51.4 & 44.2 & 43.1 & -1 \\
\hline 70 & 41.2 & 18.4 & 54.2 & 50.4 & 26.8 & 60.6 & 51.4 & 42.2 & 41.1 & -3 \\
\hline 71 & 41.2 & 18.4 & 46.2 & 48.4 & 24.8 & 62.6 & 50.4 & 44.2 & 41.1 & -9 \\
\hline 72 & 49.2 & 17.4 & 54.2 & 50.4 & 18.8 & 66.6 & 50.4 & 50.2 & 43.1 & -7 \\
\hline 73 & 43.2 & 18.4 & 54.2 & 46.4 & 24.8 & 66.6 & 51.4 & 48.2 & 43.1 & -9 \\
\hline 74 & 43.2 & 20.4 & 46.2 & 50.4 & 26.8 & 66.6 & 52.4 & 44.2 & 35.1 & -7 \\
\hline 75 & 49.2 & 17.4 & 46.2 & 47.4 & 26.8 & 68.6 & 50.4 & 50.2 & 35.1 & -9 \\
\hline 76 & 49.2 & 20.4 & 52.2 & 48.4 & 24.8 & 68.6 & 52.4 & 44.2 & 39.1 & -7 \\
\hline 77 & 47.2 & 17.4 & 54.2 & 50.4 & 26.8 & 60.6 & 54.4 & 44.2 & 35.1 & -9 \\
\hline
\end{tabular}

Table 6.3: Design of Experiment generated from the ten design variables selected. 


\begin{tabular}{|c|c|c|c|c|c|c|c|}
\hline $\begin{array}{c}\text { A } \\
\text { Hub L.E. } \\
\text { (Degrees) }\end{array}$ & $\begin{array}{c}\text { B } \\
\text { Hub M.C. } \\
\text { (Degrees) }\end{array}$ & $\begin{array}{c}\text { D } \\
\text { Mid L.E. } \\
\text { (Degrees) }\end{array}$ & $\begin{array}{c}\text { E } \\
\text { Mid M.C } \\
\text { (Degrees) }\end{array}$ & $\begin{array}{c}\mathrm{F} \\
\text { Mid T.E } \\
\text { (Degrees) }\end{array}$ & $\begin{array}{c}\text { G } \\
\text { Tip L.E } \\
\text { (Degrees) }\end{array}$ & $\begin{array}{c}\mathrm{H} \\
\text { Tip M.C } \\
\text { (Degrees) }\end{array}$ & $\begin{array}{c}\text { I } \\
\text { Tip L.E } \\
\text { (Degrees) }\end{array}$ \\
\hline 41.2 & 21.4 & 51.152 & 50.4 & 26.8 & 60.6 & 50.4 & 42.2 \\
\hline
\end{tabular}

Table 6.4: Optimized values of rotor design variables used in comprehensive application of methodology.

\begin{tabular}{|c|c|}
\hline $\begin{array}{c}\text { M } \\
\text { Mid L.E }\end{array}$ & $\begin{array}{c}\text { O } \\
\text { Mid T.E } \\
\text { (Degrees) }\end{array}$ \\
\hline $\mathbf{3 5 . 1}$ & $\mathbf{- 2 . 9 9}$ \\
\hline
\end{tabular}

Table 6.5: Optimized values of stator design variables used in comprehensive application of methodology.

\begin{tabular}{|c|c|c|c|}
\hline & $\begin{array}{c}\text { Total } \\
\text { Pressure } \\
\text { Ratio }\end{array}$ & $\begin{array}{c}\text { Adiabatic } \\
\text { Efficiency }\end{array}$ & $\begin{array}{c}\text { RWIC } \\
\text { (SPL) }\end{array}$ \\
\hline Design & $\mathbf{1 . 8 6 5 7}$ & $\mathbf{0 . 8 3 5 6}$ & $\mathbf{6 1 . 8 0}$ \\
\hline CFD & $\mathbf{1 . 8 2 5 5}$ & $\mathbf{0 . 8 1 5 2}$ & $\mathbf{6 3 . 1 4}$ \\
\hline \% Diff & $\mathbf{2 . 1 5}$ & $\mathbf{2 . 4 4}$ & $\mathbf{2 . 1 7}$ \\
\hline
\end{tabular}

Table 6.6: Differences observed between predicted and computed values for the three objective functions in comprehensive application of methodology. 


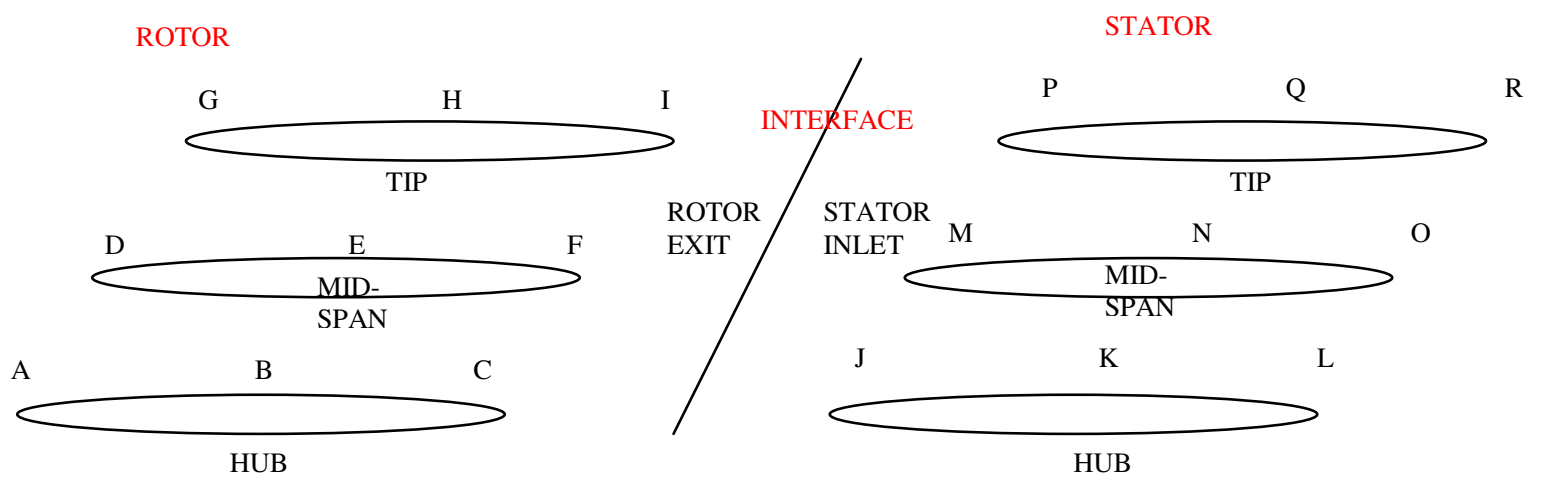

Figure 6.1: 18 design variables (camber angles) used for the comprehensive application of methodology.

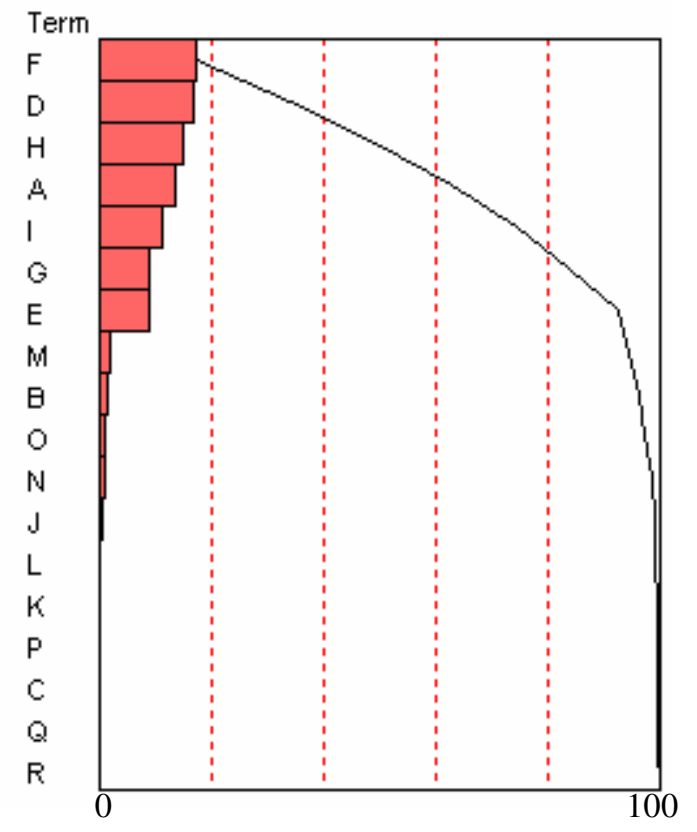

Figure 6.2: Pareto curve obtained with total pressure ratio as the objective function. 


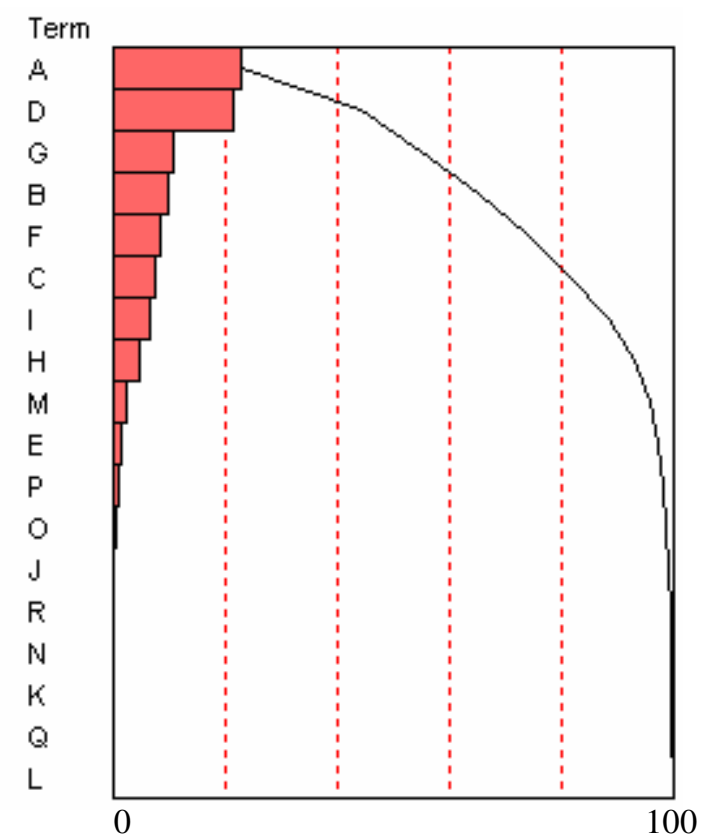

Figure 6.3: Pareto curve obtained with adiabatic efficiency as the objective function.

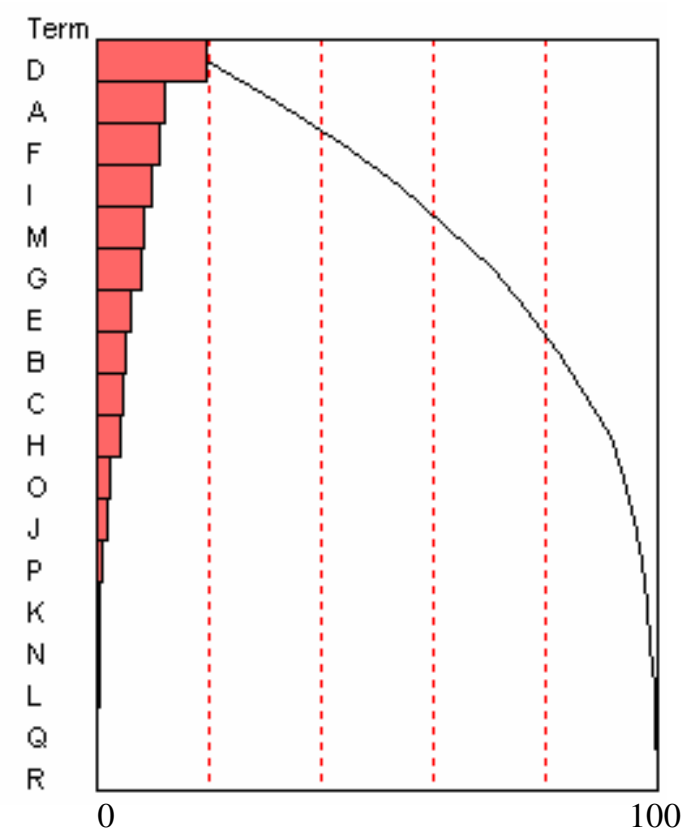

Figure 6.4: Pareto curve obtained with rotor wake influence coefficient (RWIC) as the objective function. 

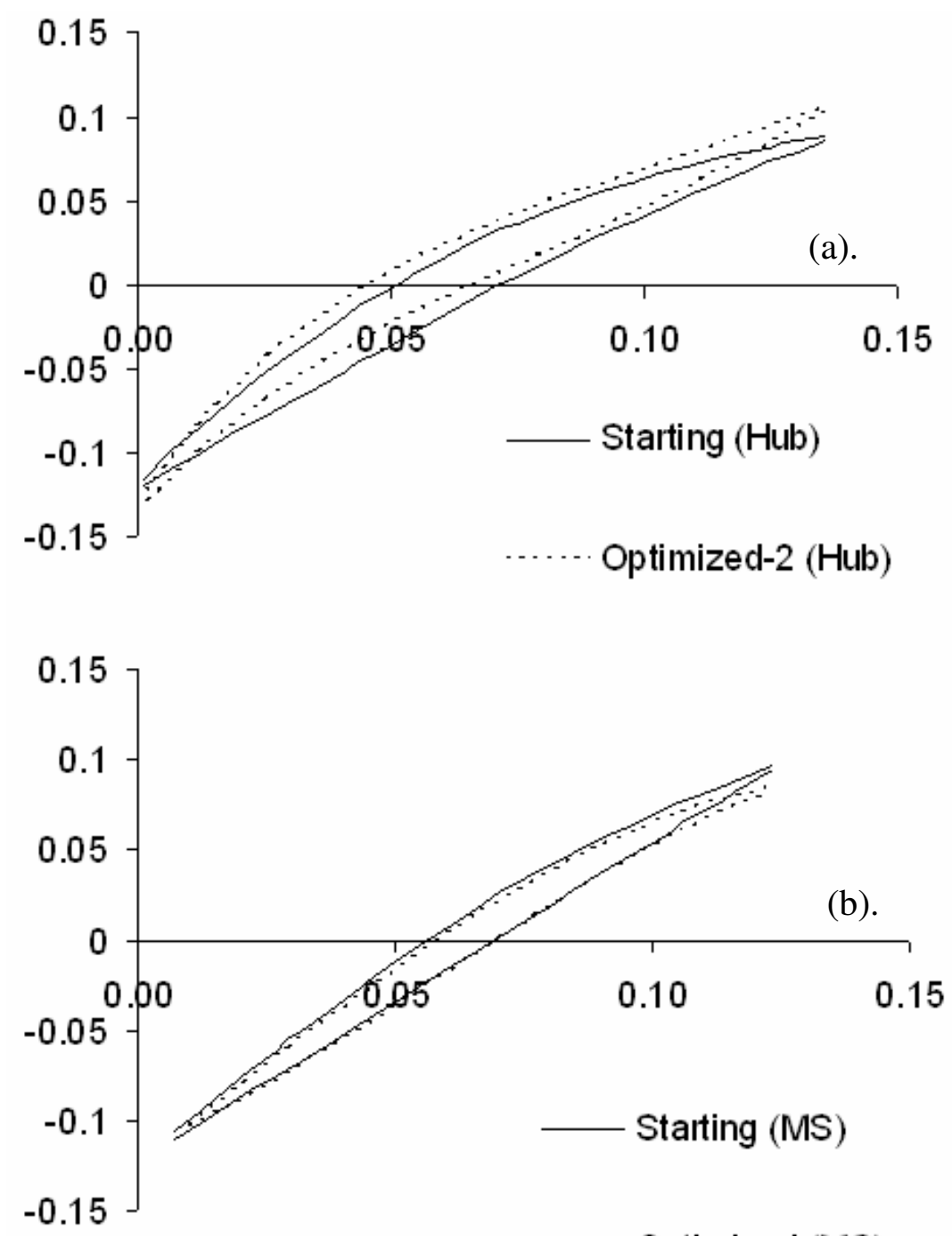

Optimized (MS)

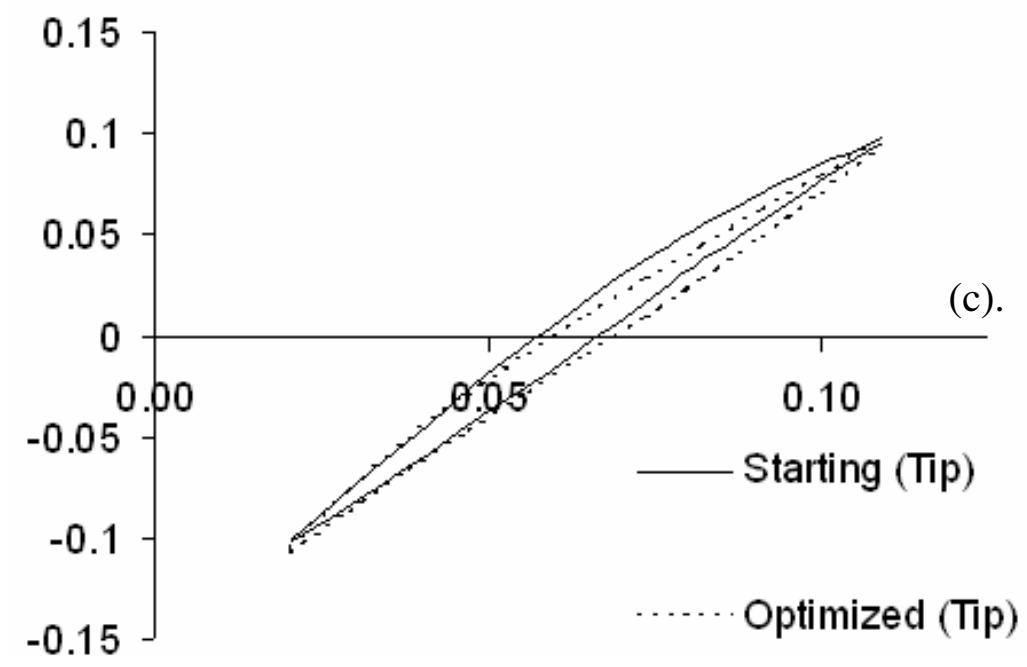

Figure 6.5: Rotor blade profile comparisons between the starting and optimized configurations at (a) hub, (b) mid-span and (c) tip locations. 

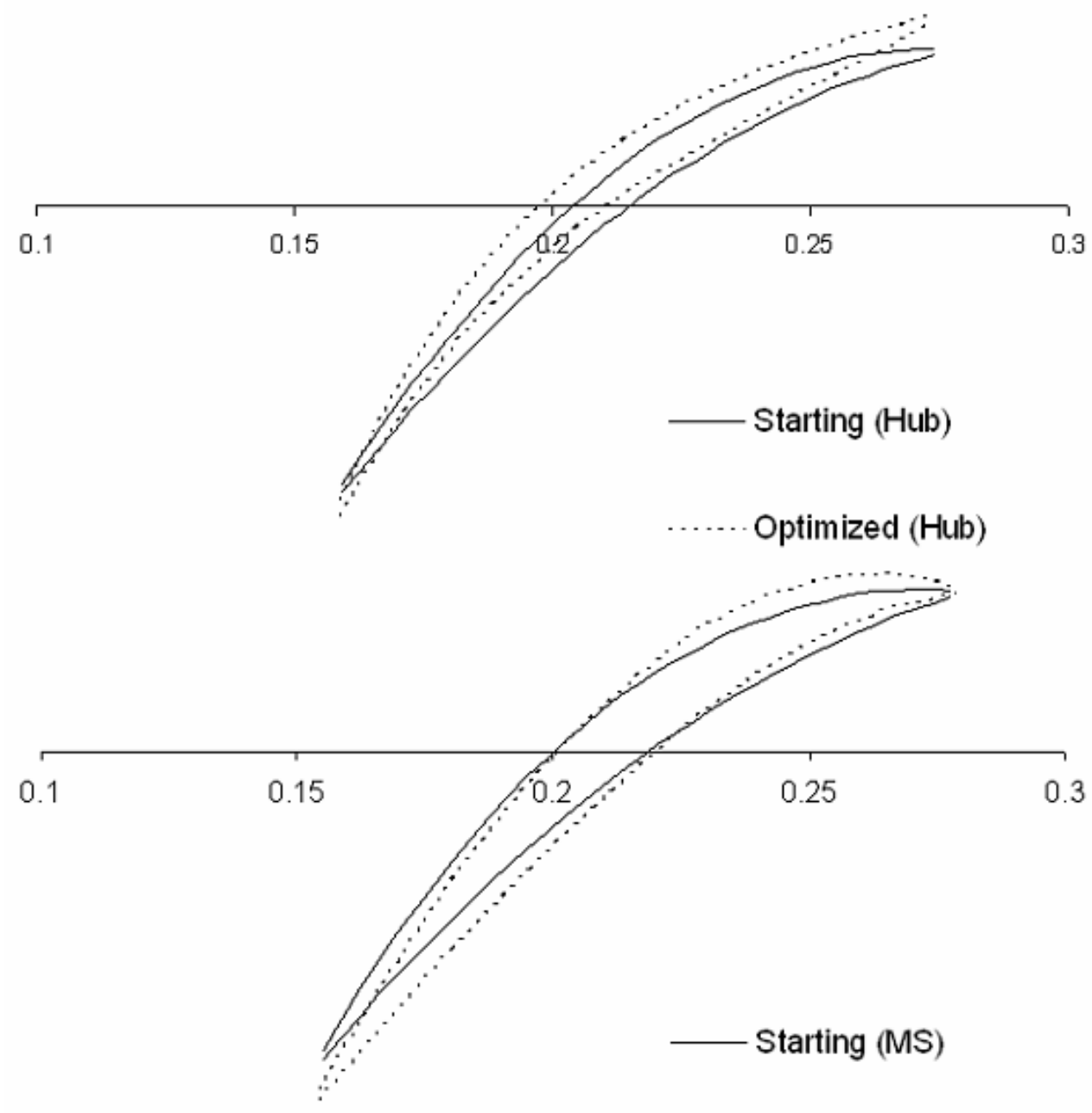

...... Optimized (MS)

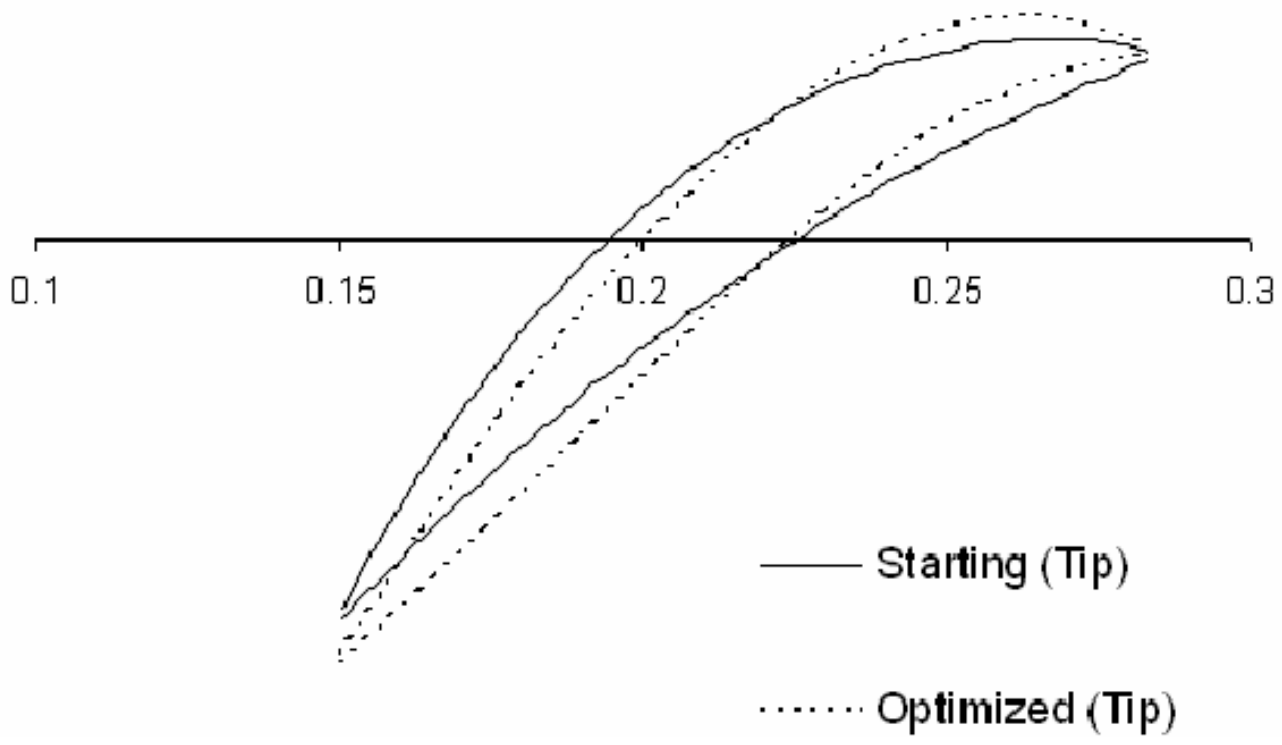

Figure 6.6: Stator blade profile comparisons between starting and optimized configurations at (a) hub, (b) mid-span and (c) tip locations. 

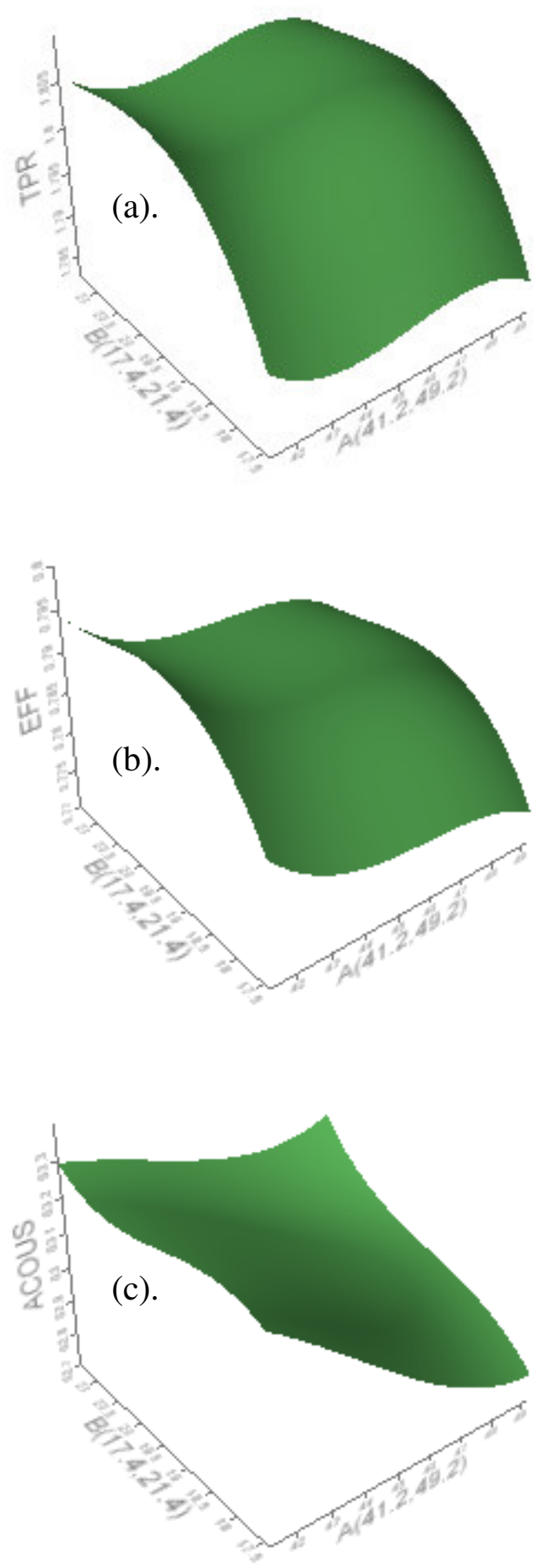

Figure 6.7: Response surface for objective functions a). total pressure ratio, b). adiabatic efficiency and c). rotor wake influence coefficient, as a function of design variables $A$ and $B$. 

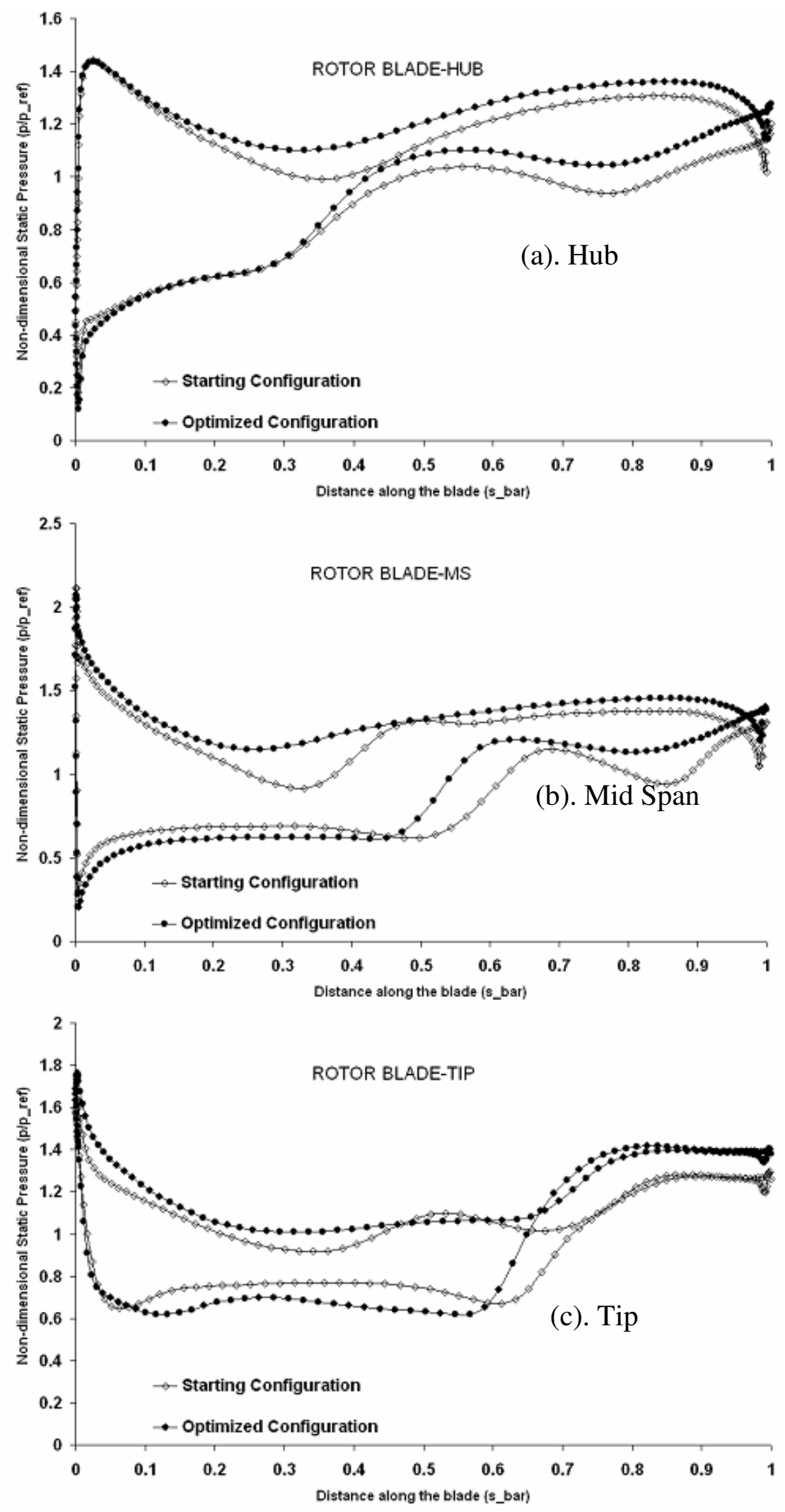

Figure 6.8: Rotor blade loading curve comparison at (a) Hub, (b) Mid Span and (c) Tip between the starting configuration and optimized configuration. 

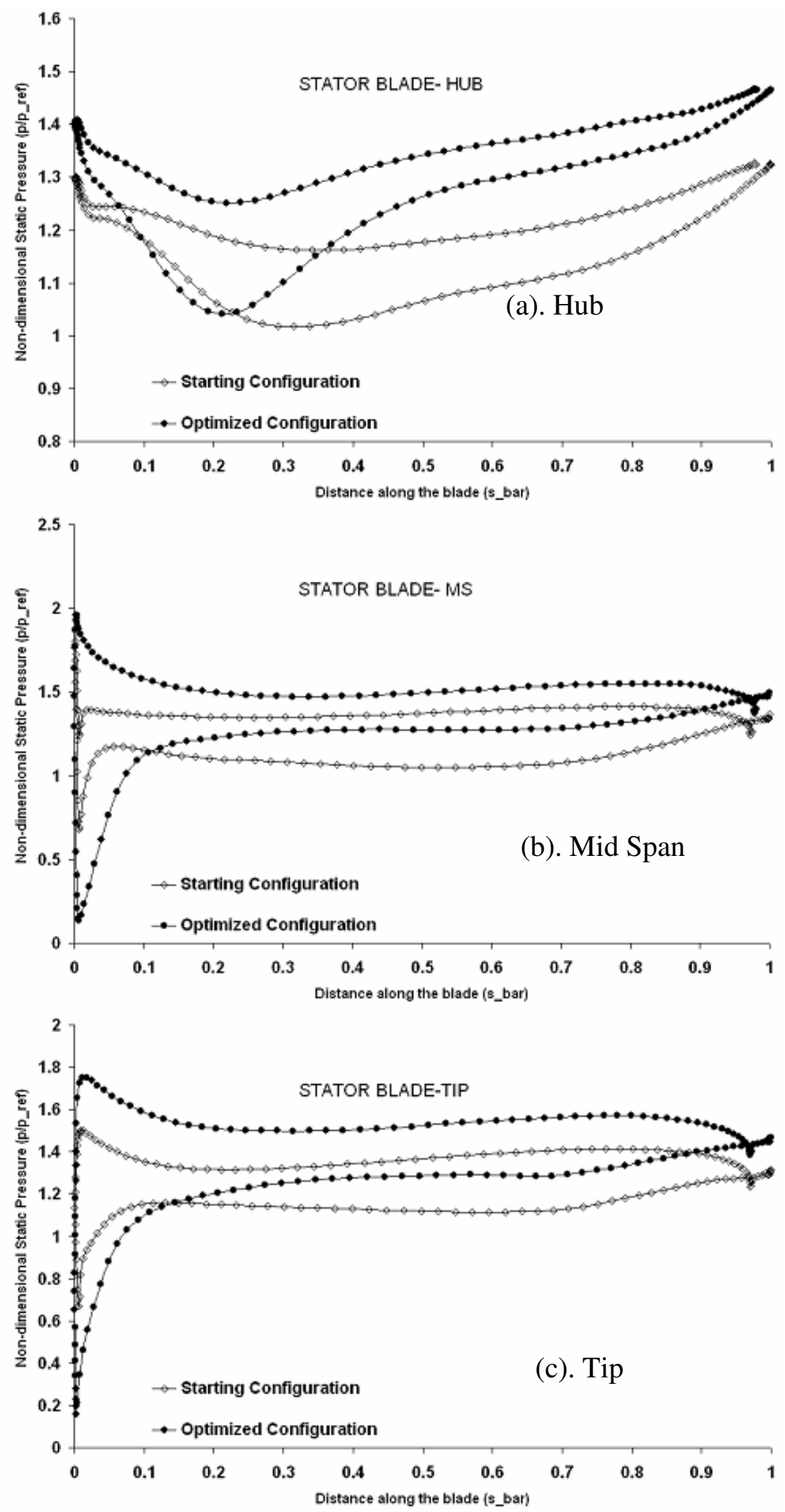

Figure 6.9: Stator blade loading curve comparison at (a) Hub, (b) Mid Span and (c) Tip between the starting configuration and optimized configuration. 


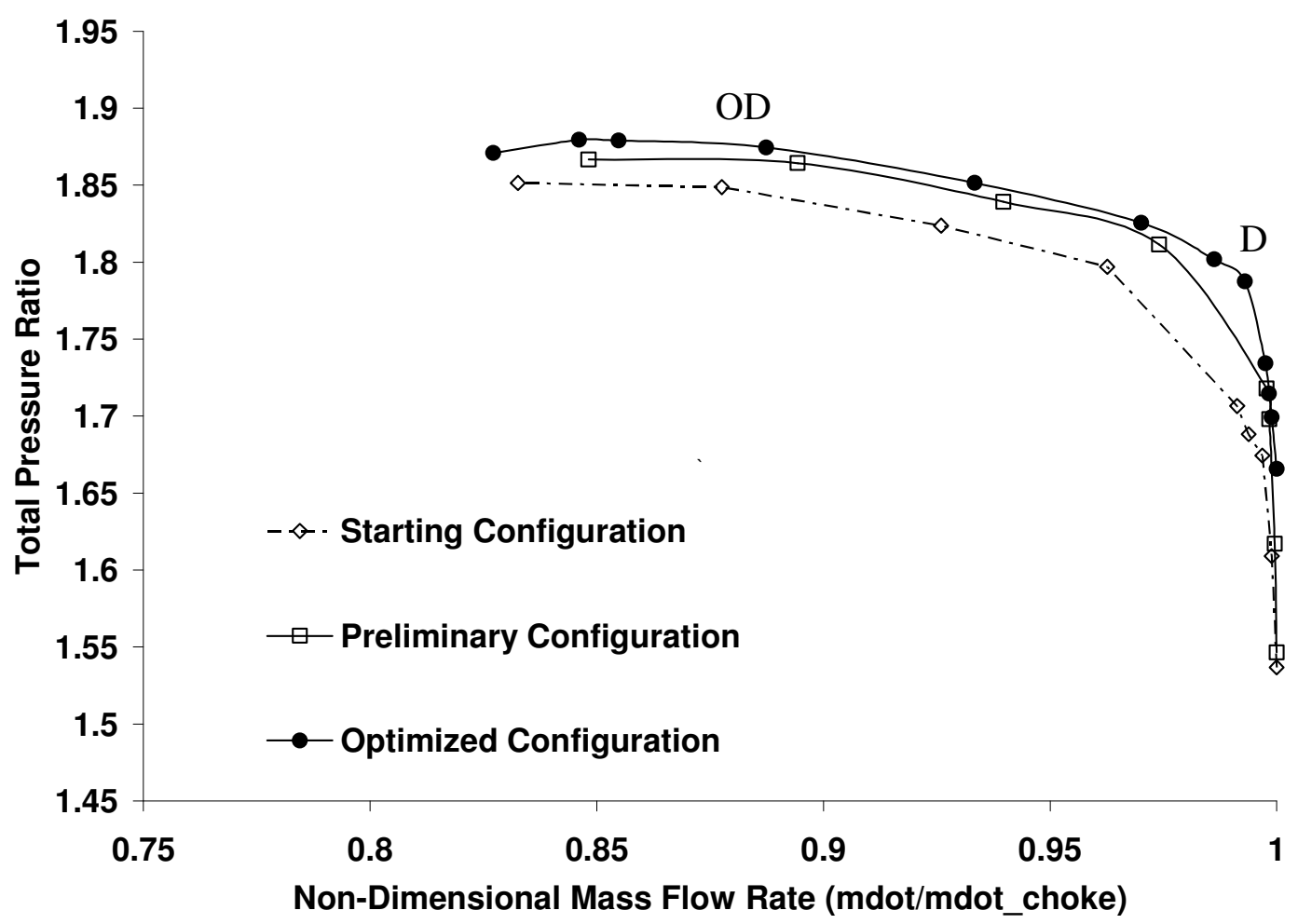

Figure 6.10: Performance map comparing the Starting configuration to the Optimized configuration.

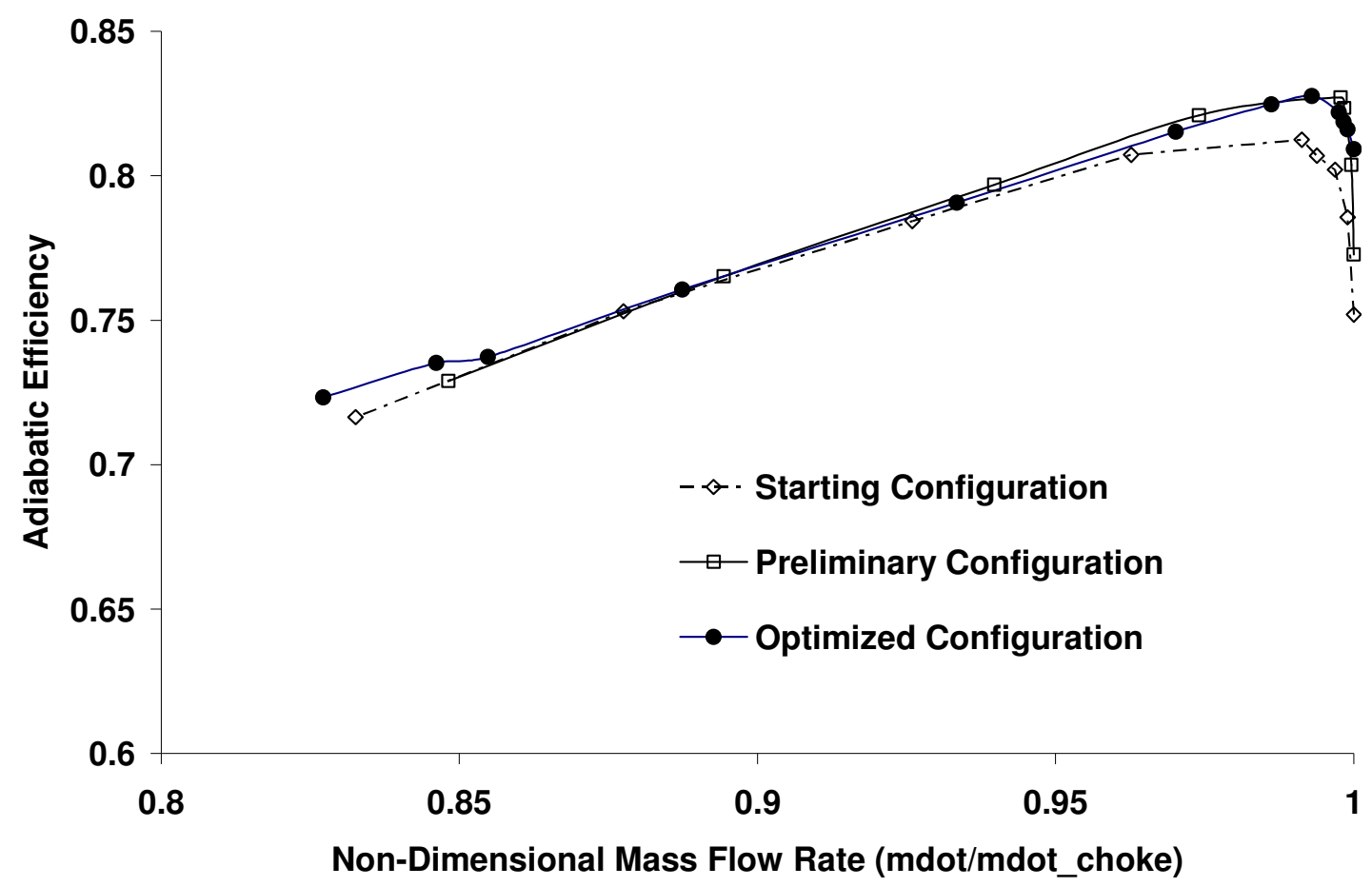

Figure 6.11: Adiabatic efficiency map comparing the Starting configuration to the Optimized configuration. 


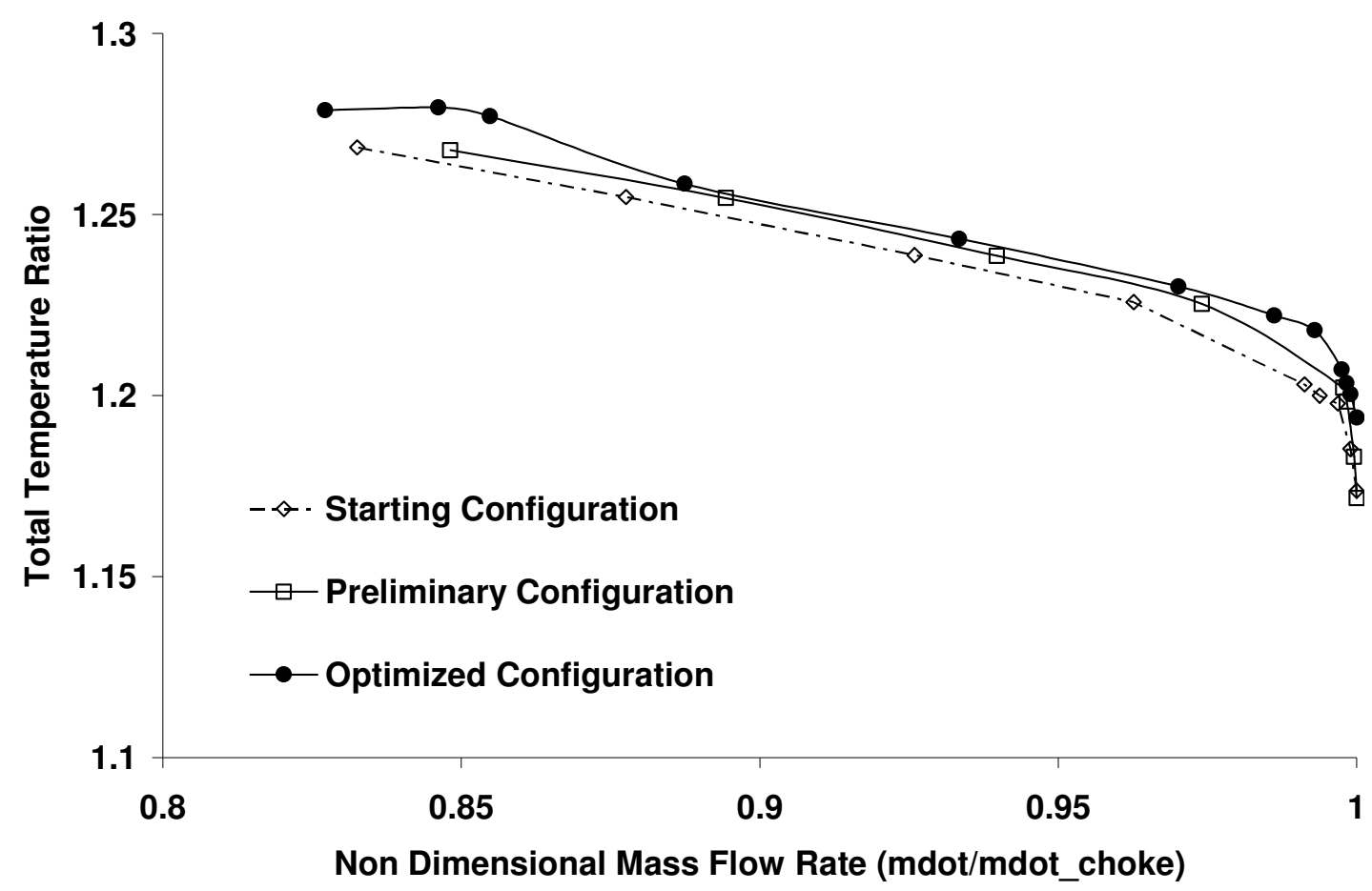

Figure 6.12: Total temperature ratio map comparing the Starting configuration to the Optimized configuration.

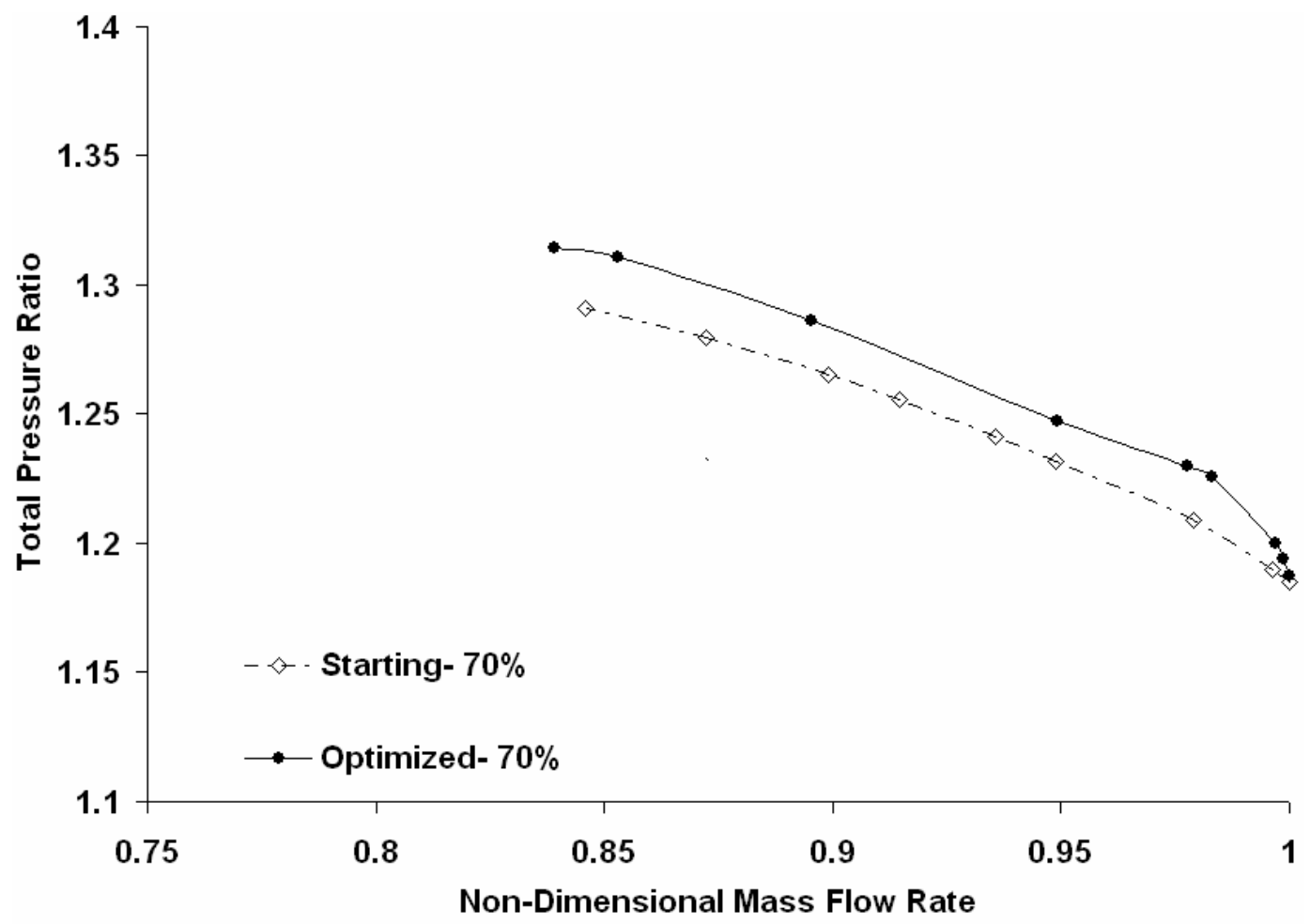

Figure 6.13: Performance maps at 70\% RPM for the Starting configuration and the Optimized configuration. 


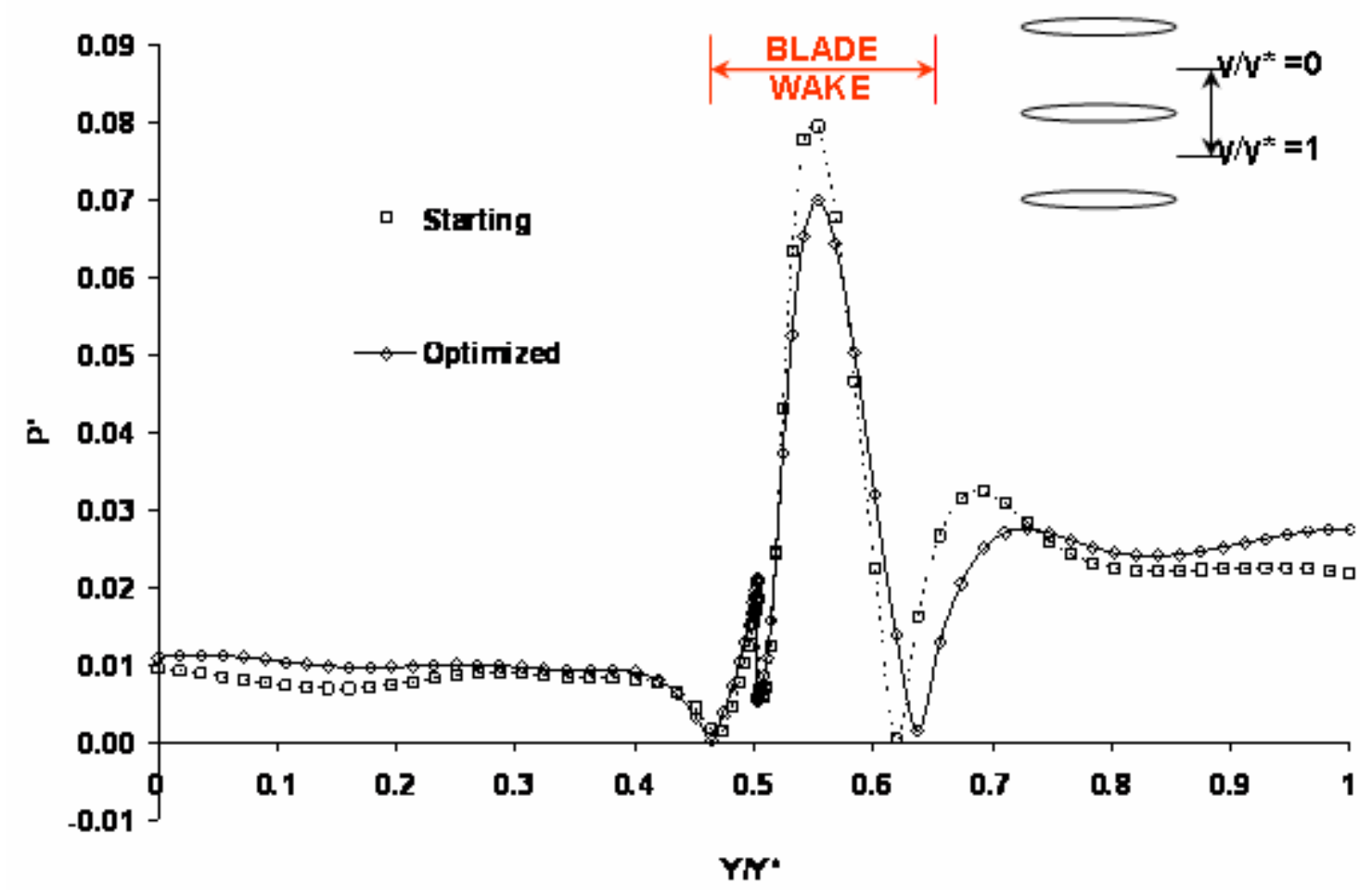

Figure 6.14: Comparisons of the blade-to-blade pressure fluctuation influencing the rotorwake/stator interactions aft of the rotor blade at mid-span.

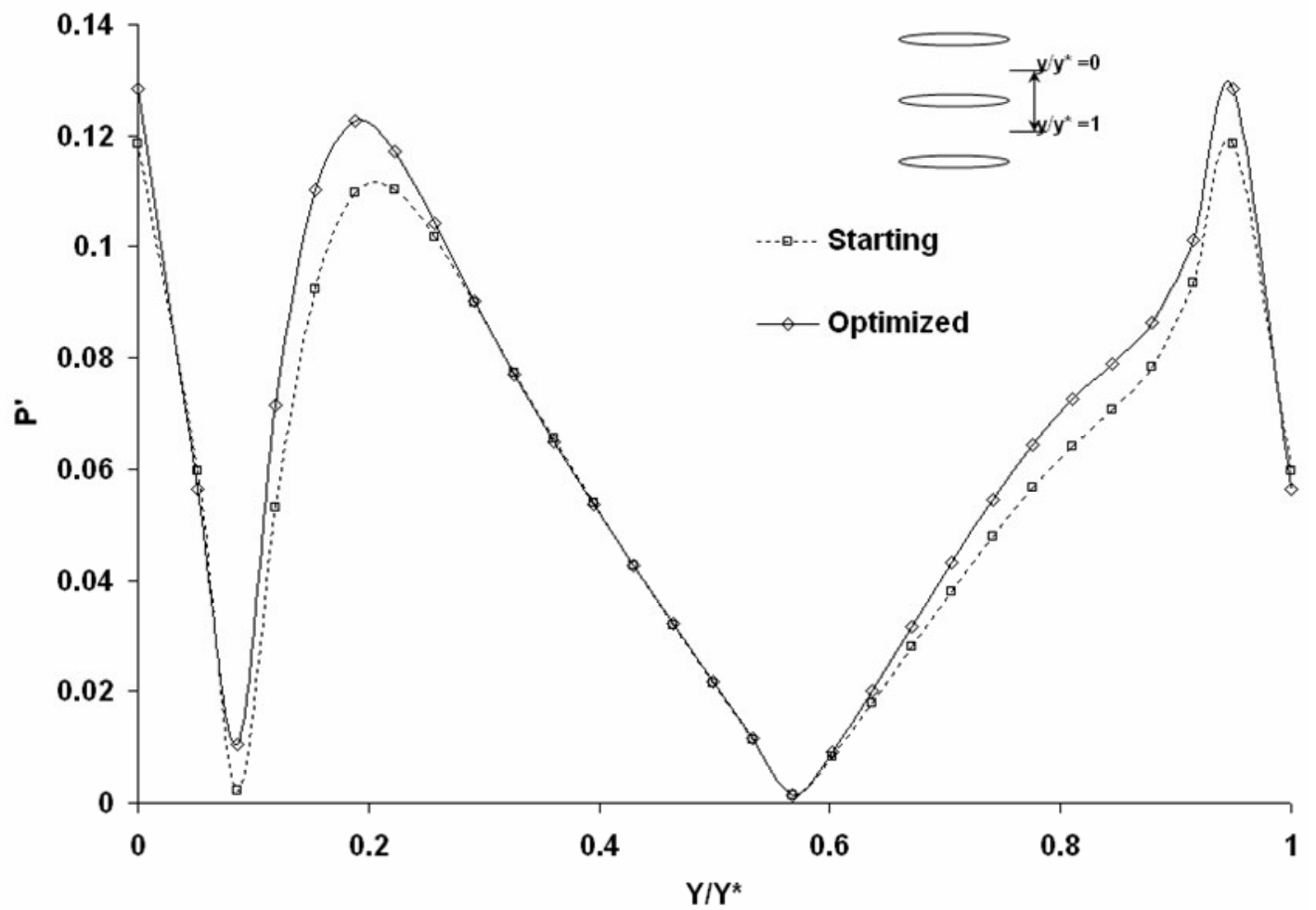

Figure 6.15: Comparisons of the blade-to-blade pressure fluctuation influencing the rotorwake/stator interactions ahead of the rotor blade at mid-span. 


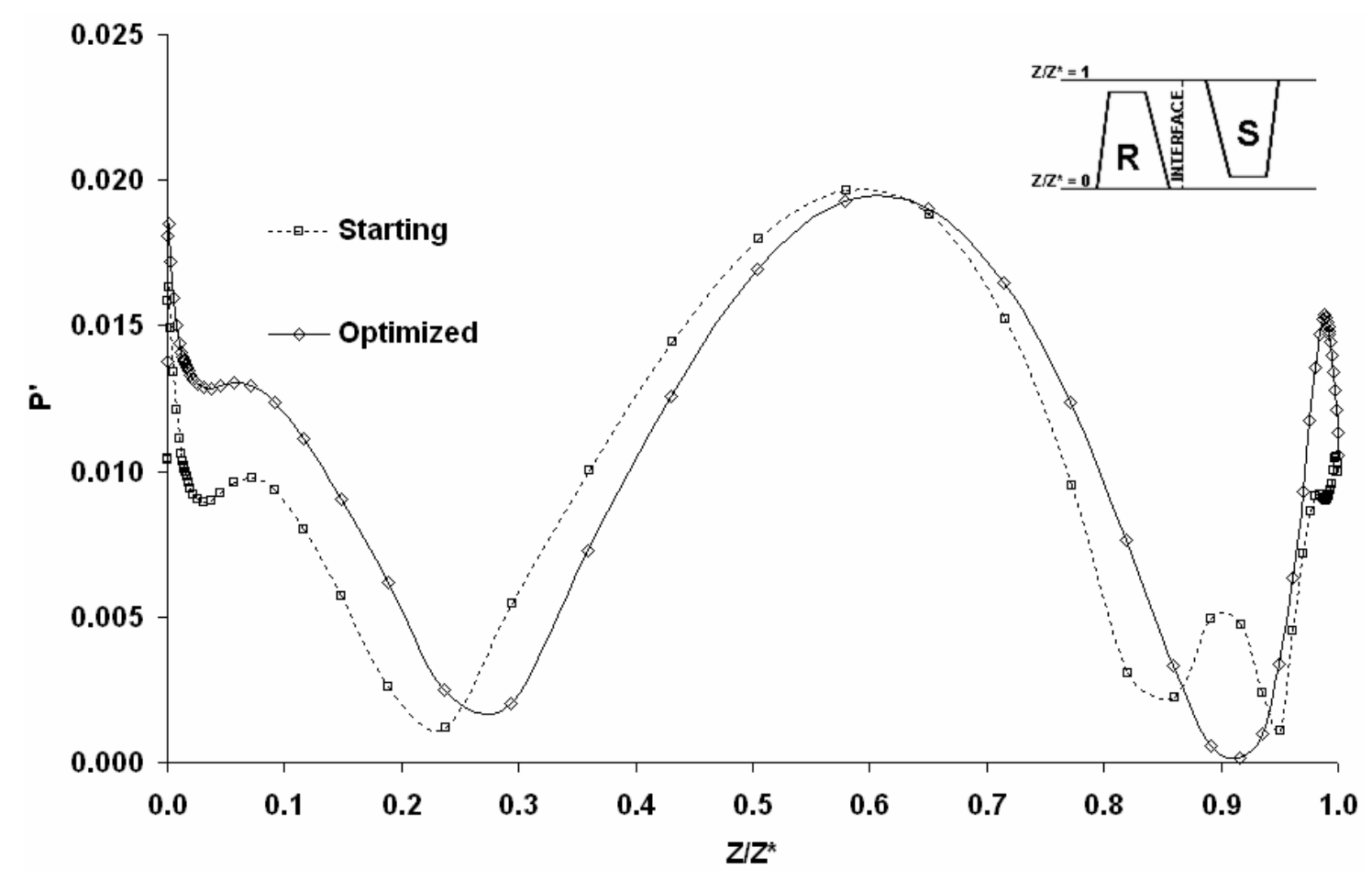

Figure 6.16: Comparisons of the hub-to-tip pressure fluctuations influencing the rotorwake/stator interactions at rotor-stator interface. 


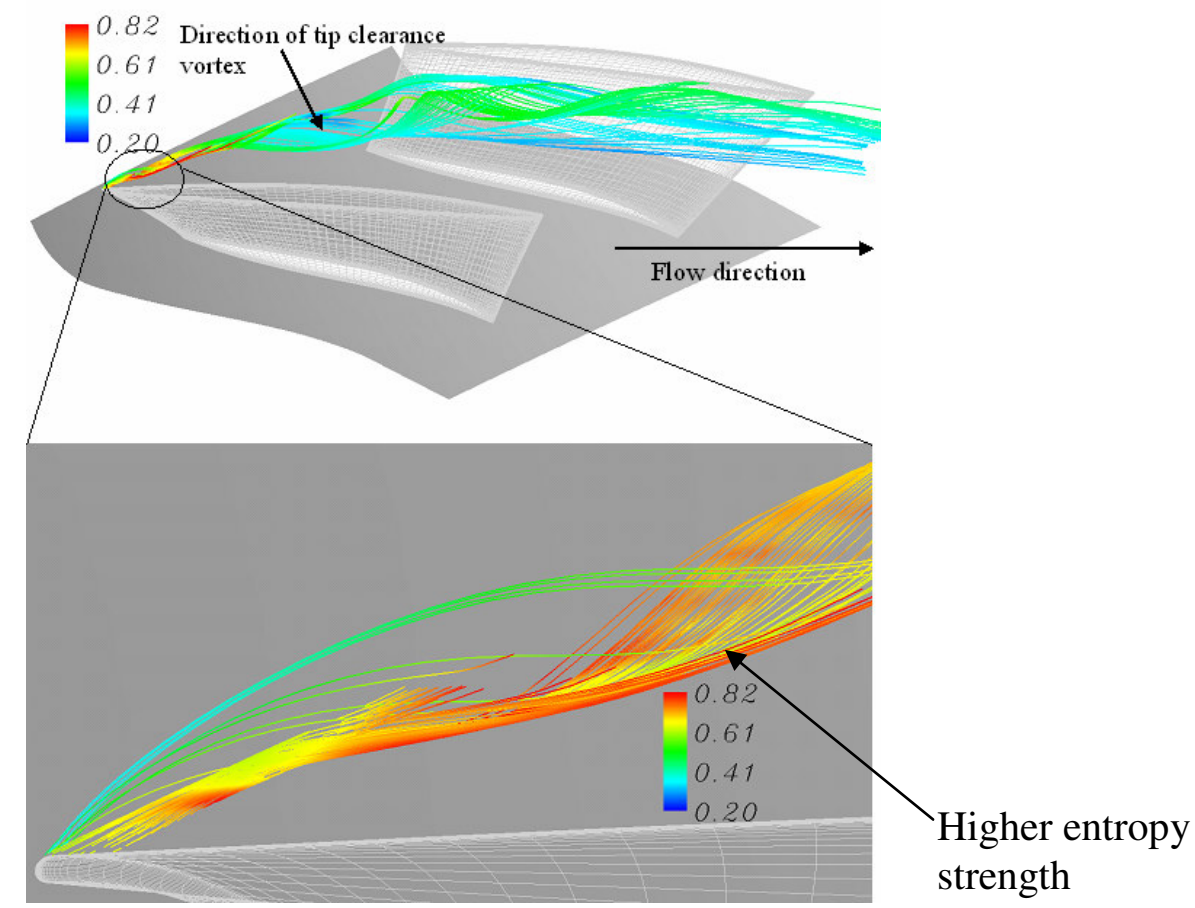

(a). Starting Configuration

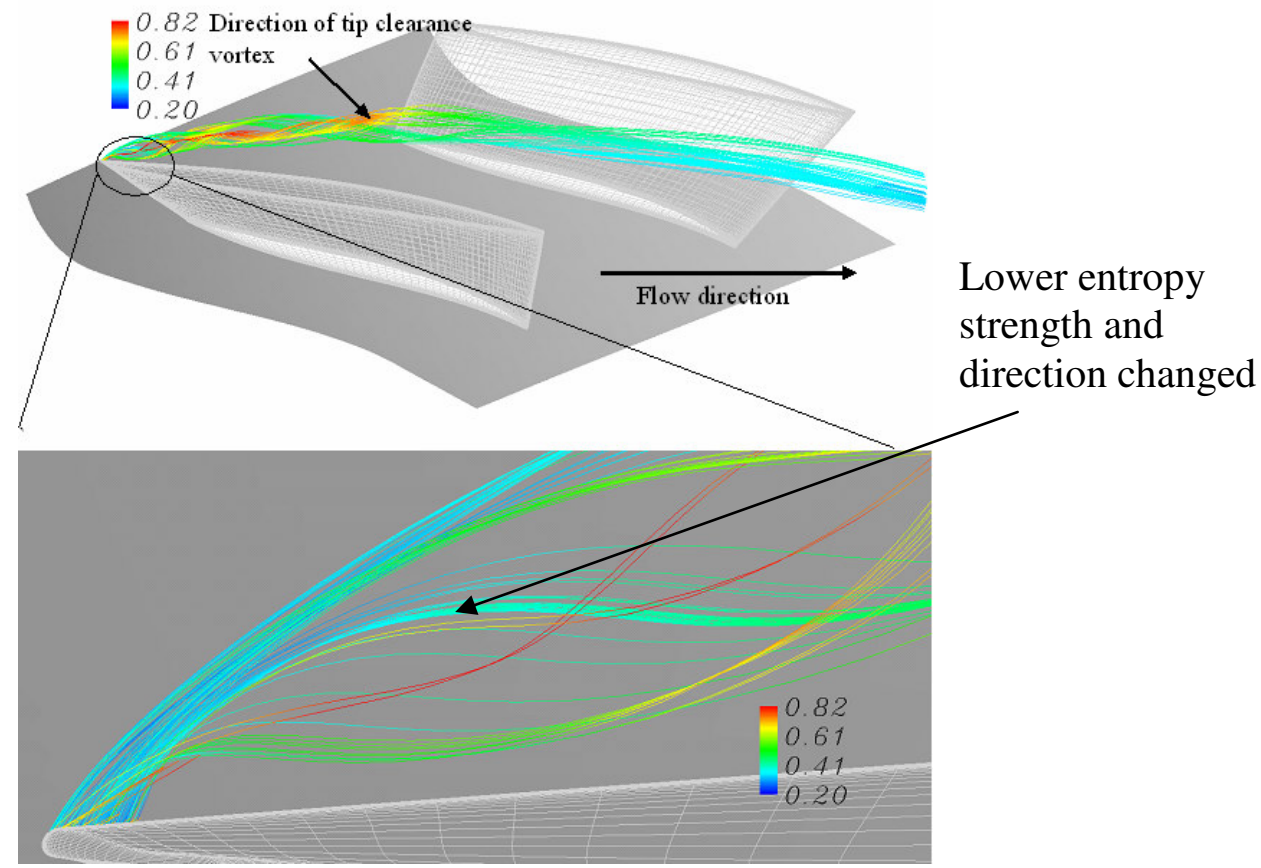

(b). Optimized Configuration

Figure 6.17: Direction and entropy strength of the rotor leading edge tip vortex. 


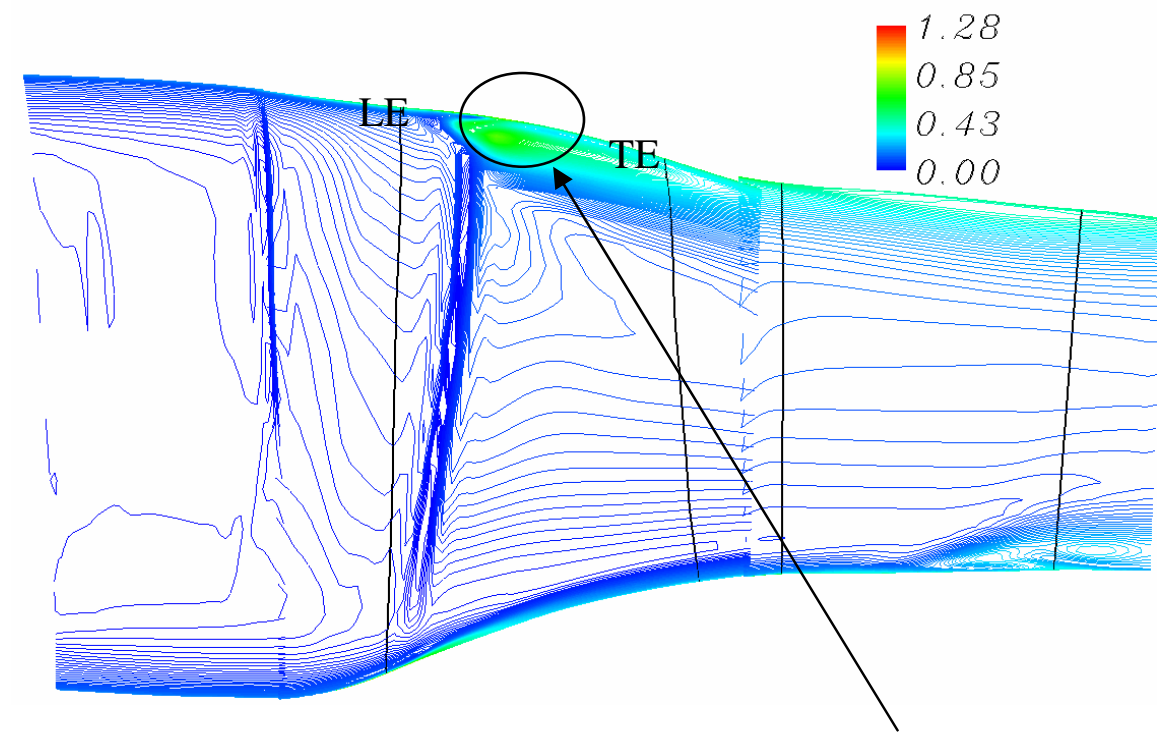

(a). Starting Configuration

Region of high

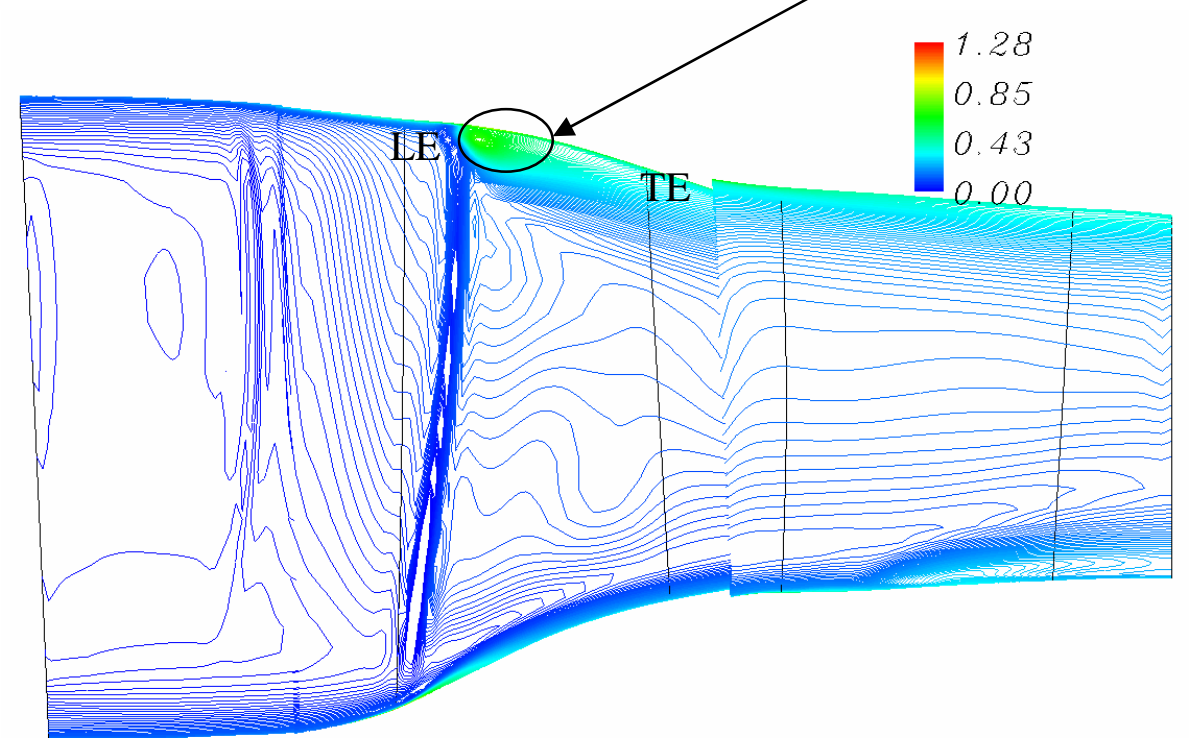

(b). Optimized Configuration

Figure 6.18: Entropy (non-dimensional) contour comparison between the (a). Starting and (b). Optimized configurations, for mid passage location at off-design condition $(\mathbf{m d o t}=$ 0.87 mdot_choke, $\left.T_{\text {ref }}=288.3 \mathrm{~K}, \rho_{\text {ref }}=1.233 \mathrm{~kg} / \mathrm{m}^{3}\right)$. 


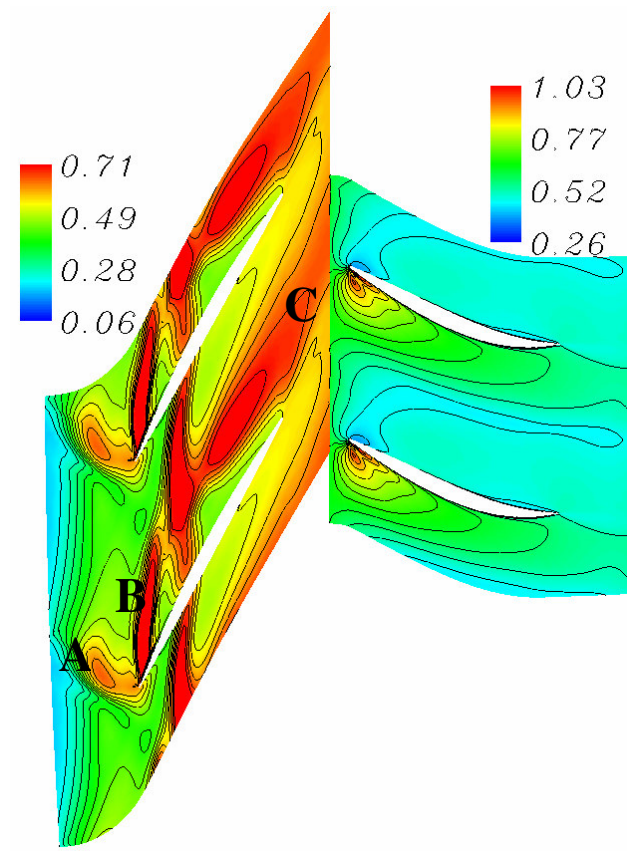

(a). Starting Configuration

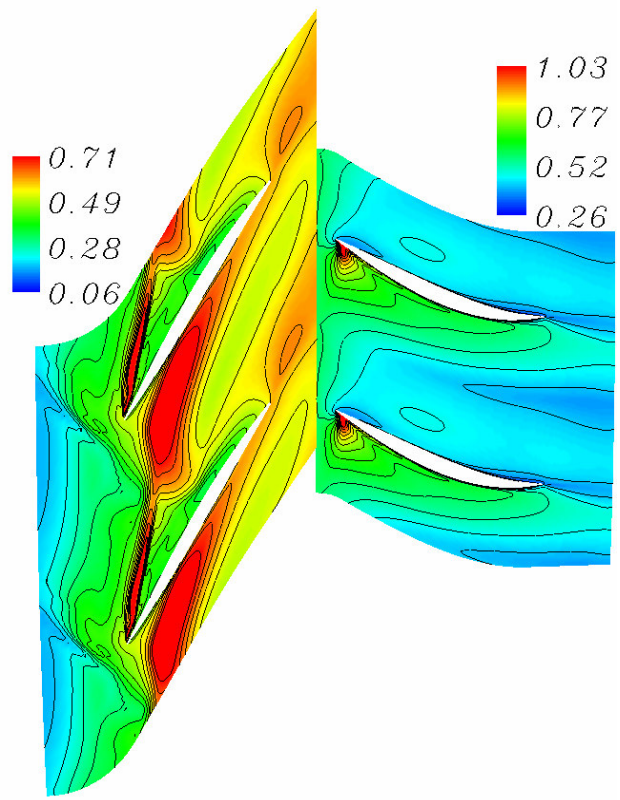

(b). Optimized Configuration

Figure 6.19: Entropy (non-dimensional) contour comparison between the (a). Starting and (b). Optimized configurations, for tip clearance section at off-design condition $(\boldsymbol{m d o t}=0.87$ mdot_choke, $T_{\text {ref }}=288.3 \mathrm{~K}, \rho_{\text {ref }}=1.233 \mathrm{~kg} / \mathrm{m}^{3}$ ). 


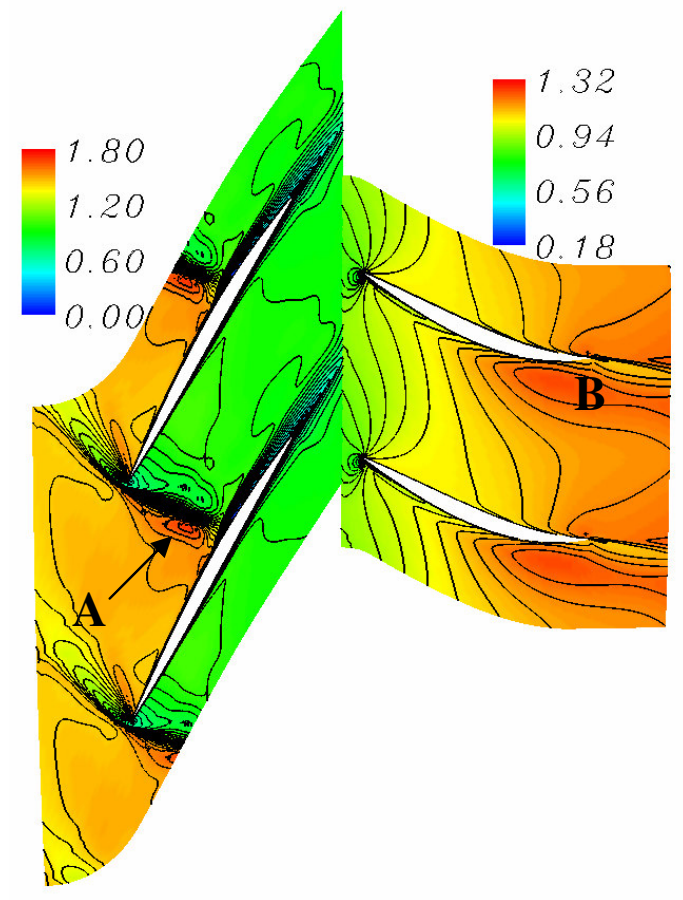

(a). Starting Configuration

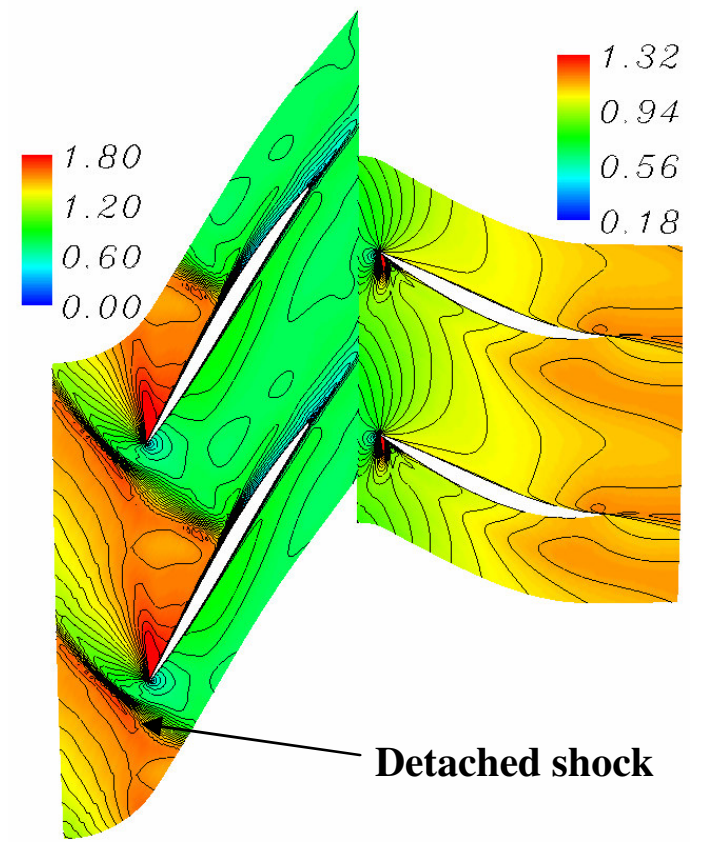

(b). Optimized Configuration

Figure 6.20: Mach number contour comparison between the (a). Starting and (b). Optimized configurations at $90 \%$ span from hub at off-design condition ( mdot $=0.87$ mdot_choke). 


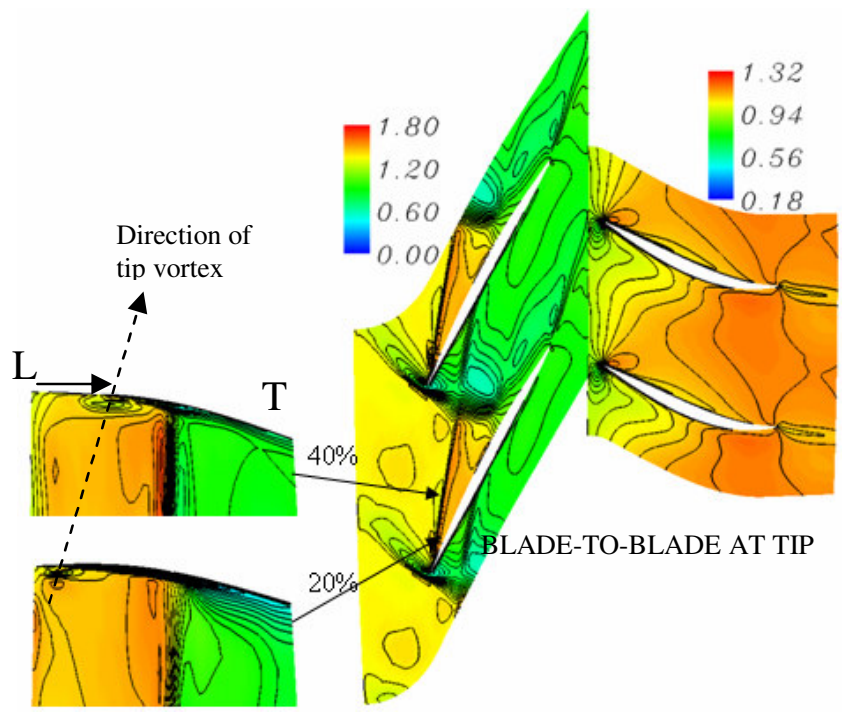

(a). Starting Configuration

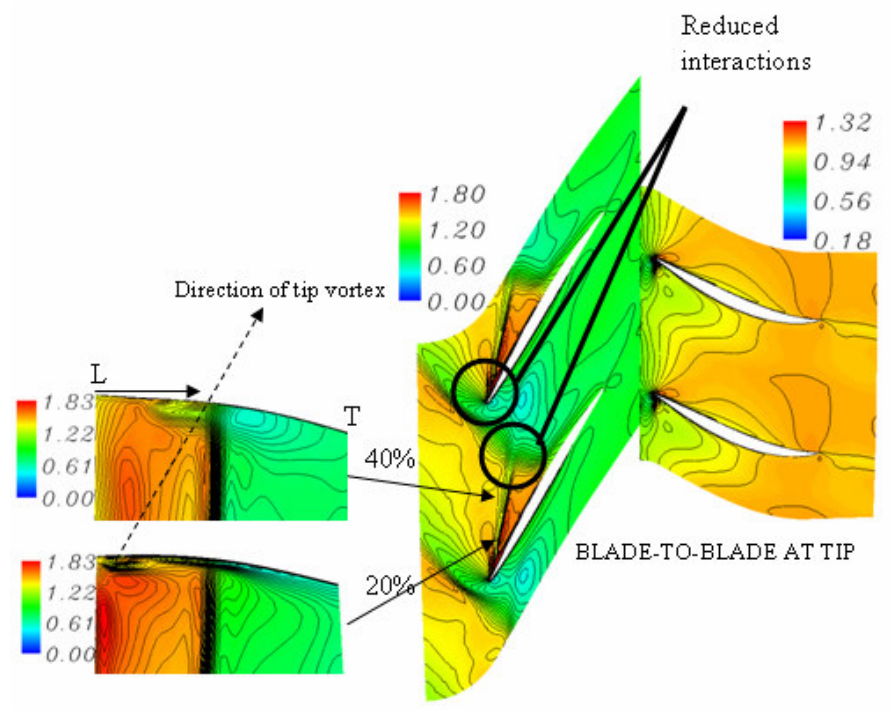

(b). Optimized Configuration

Figure 6.21: Mach number contour comparison between the (a). Starting and (b). Optimized configurations at tip off-design condition ( $\mathbf{m d o t}=\mathbf{0 . 8 7}$ mdot_choke) and two meridional planes above $70 \%$ span. 


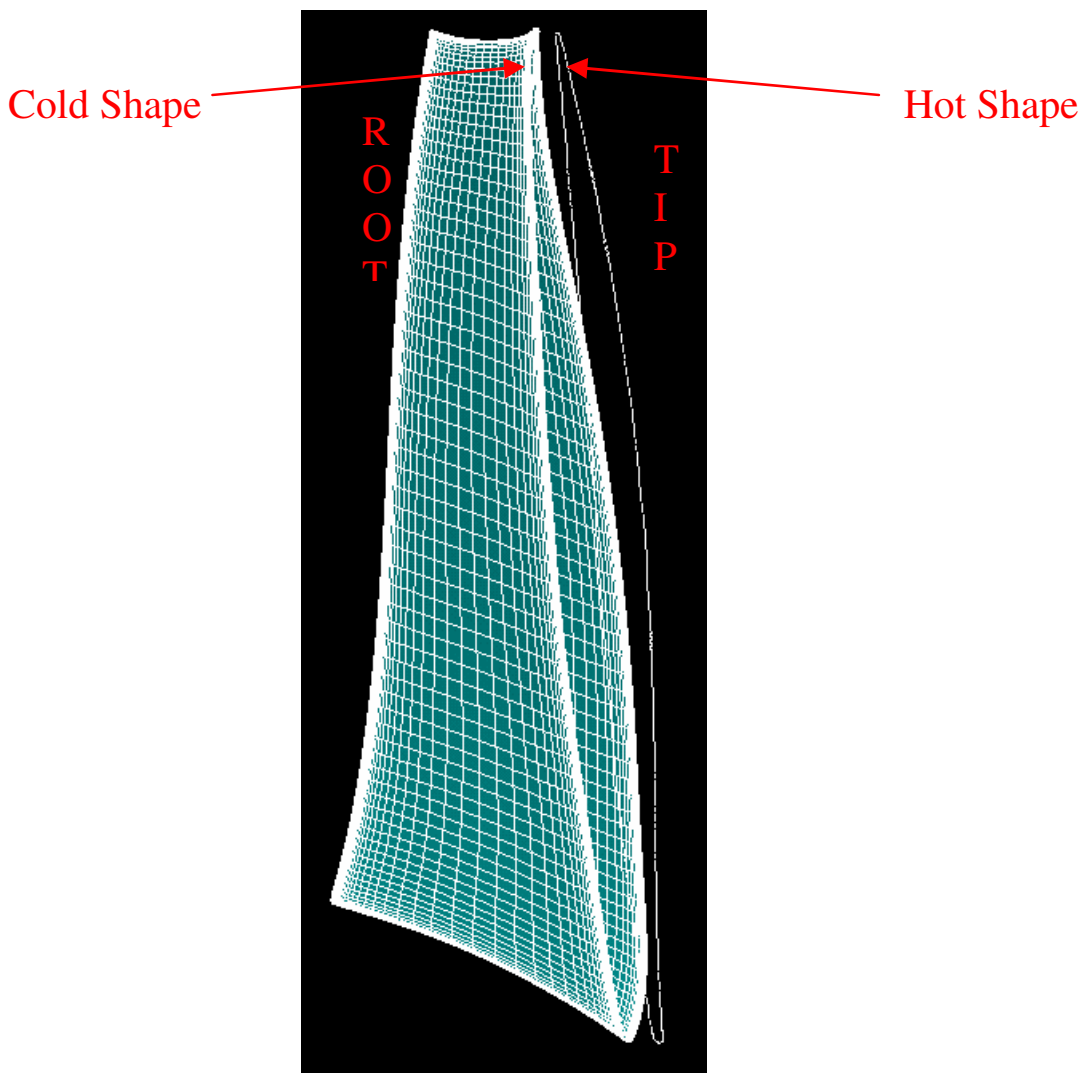

Figure 6.22: Rotor blade deflections showing the "hot" shape and the "cold" shape for the optimized configuration. 


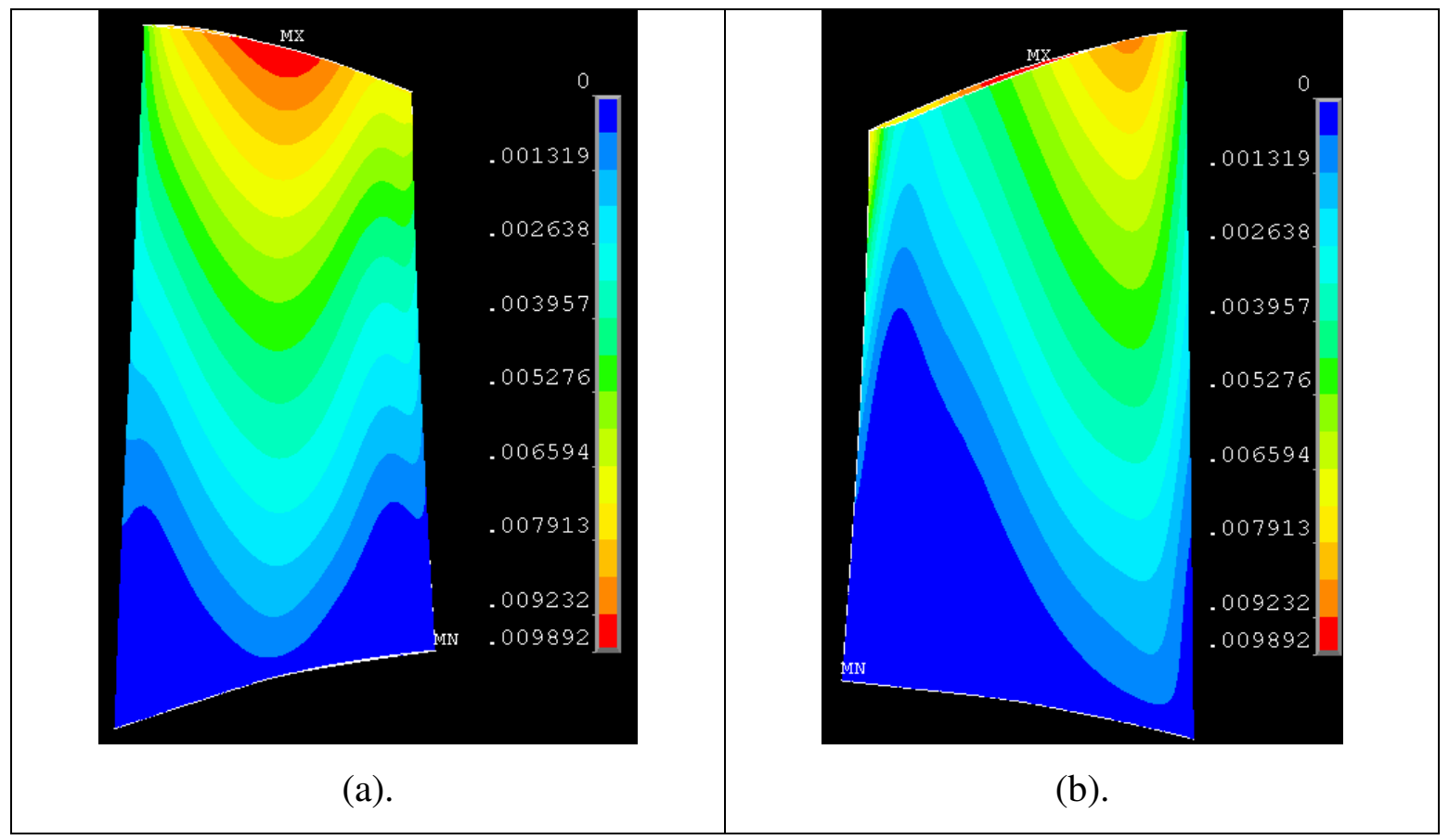

Figure 6.23: Rotor blade displacements on (a). Pressure surface and (b). Suction surface for the Optimized configuration.

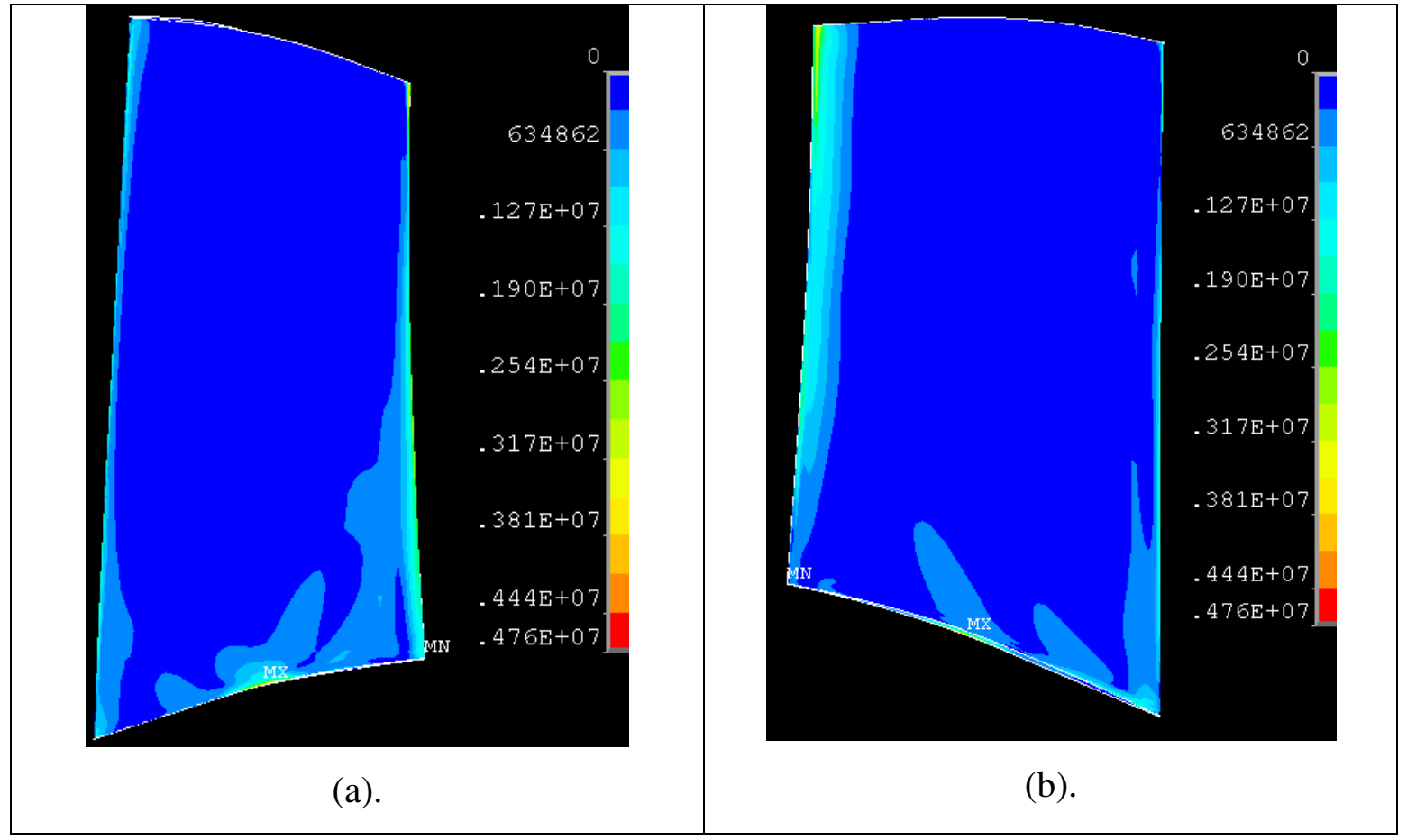

Figure 6.24: von Mises stress on rotor blade (a). Pressure surface and (b). Suction surface for the Optimized configuration. 


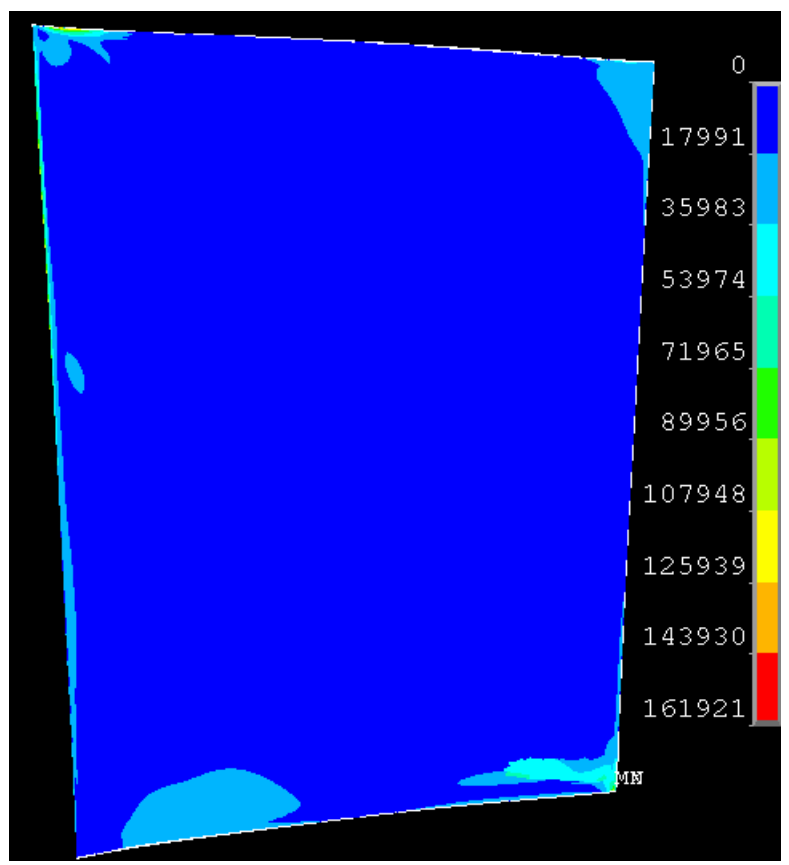

Figure 6.25: von Mises stress on stator blade suction surface for the Optimized configuration. 


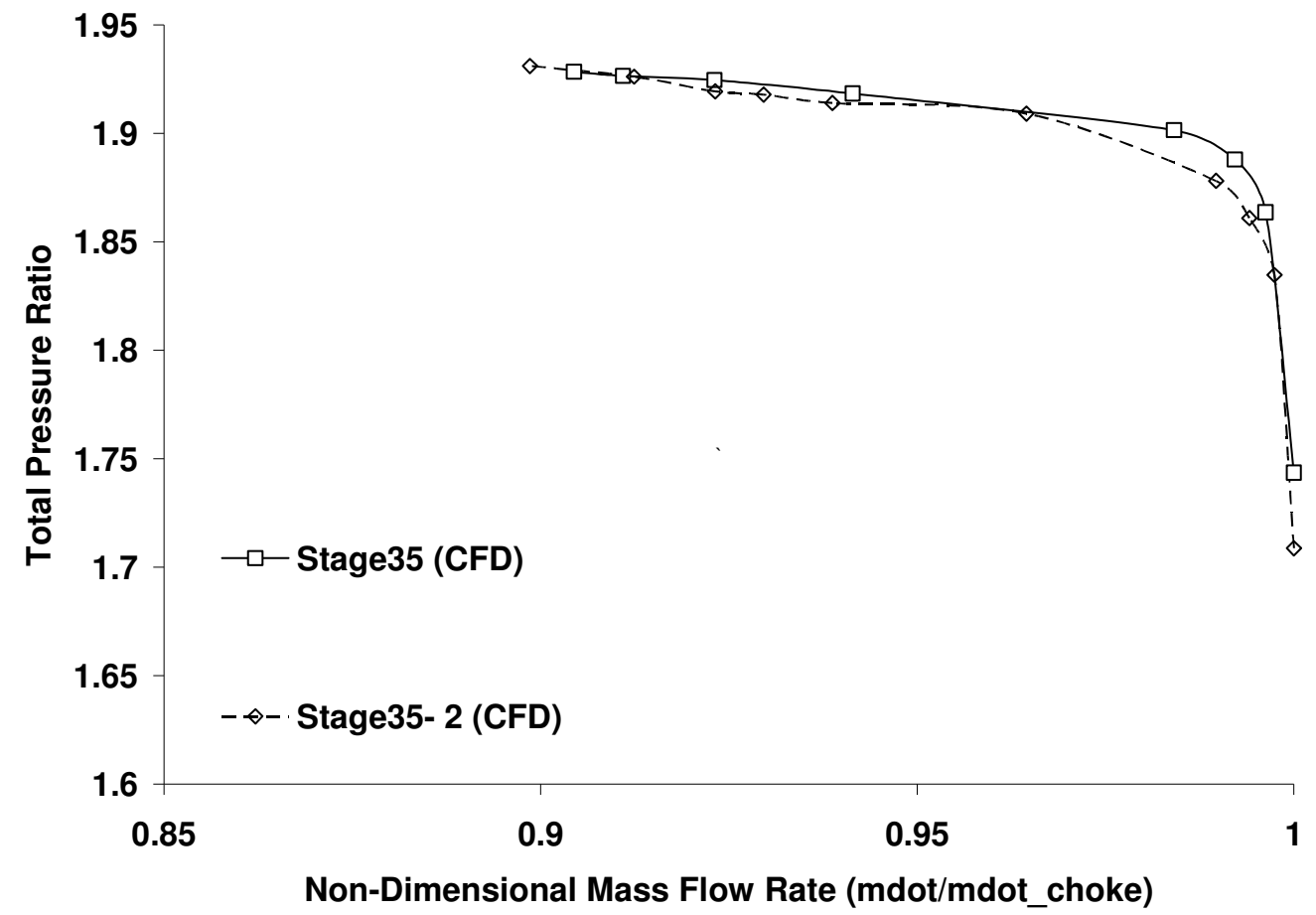

Figure 6.26: Performance map comparing the baseline Stage 35 configuration to the Stage35-2 configuration.

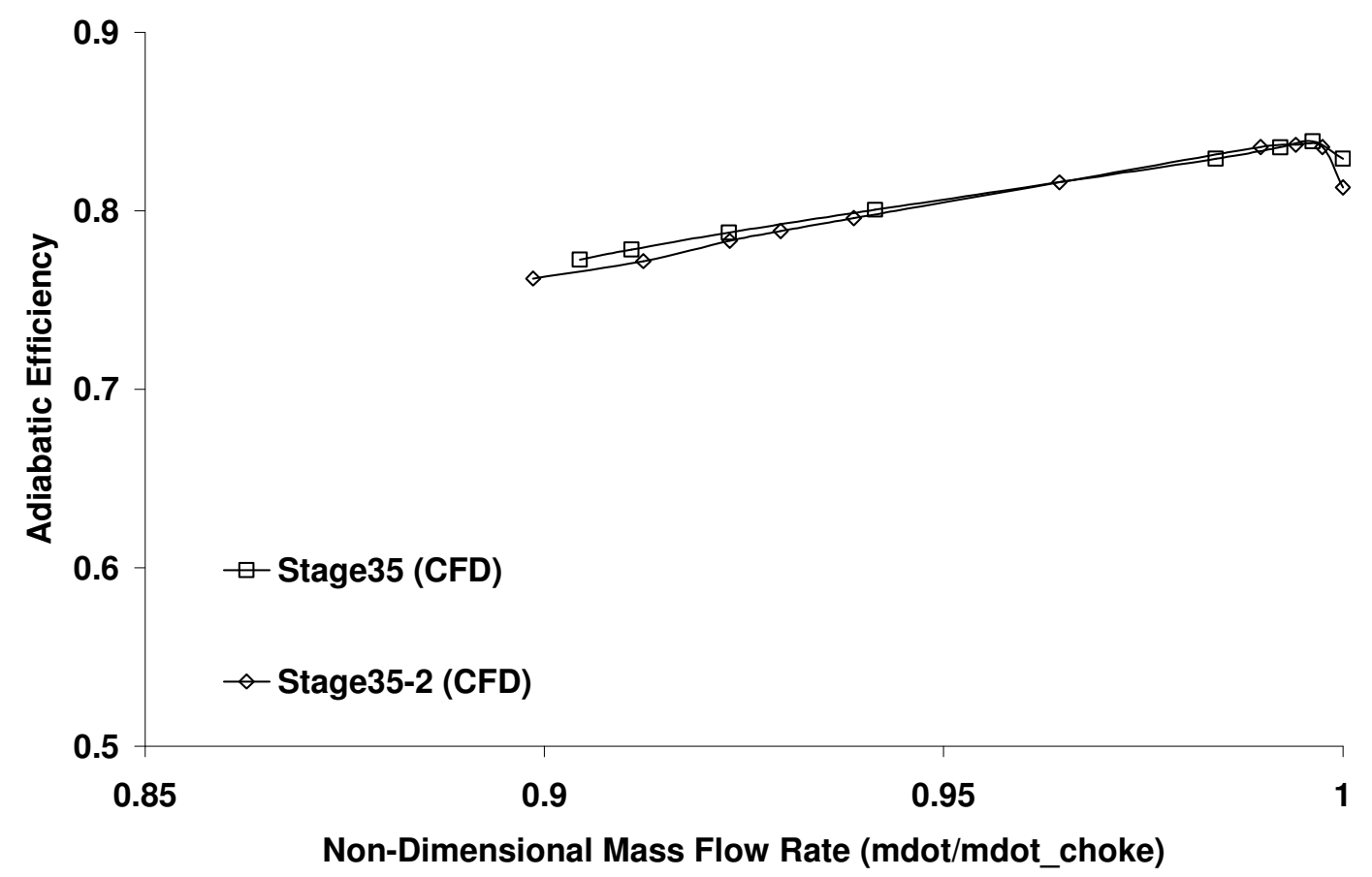

Figure 6.27: Adiabatic efficiency map comparing the baseline Stage 35 configuration to the Stage35-2 configuration. 


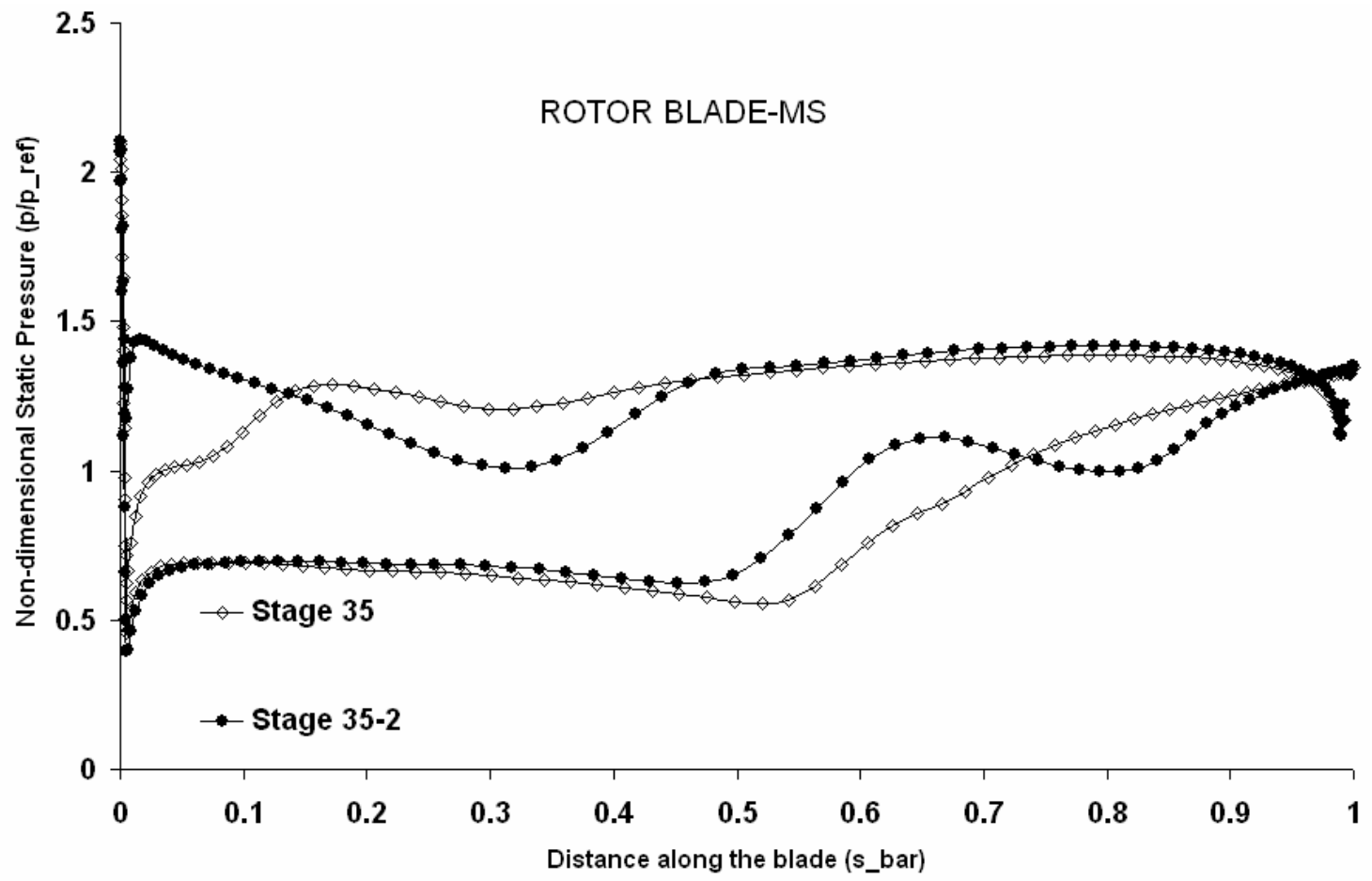

Figure 6.28: Rotor blade loading curve comparison at mid span between the Stage 35 configuration and Stage 35-2 configuration.

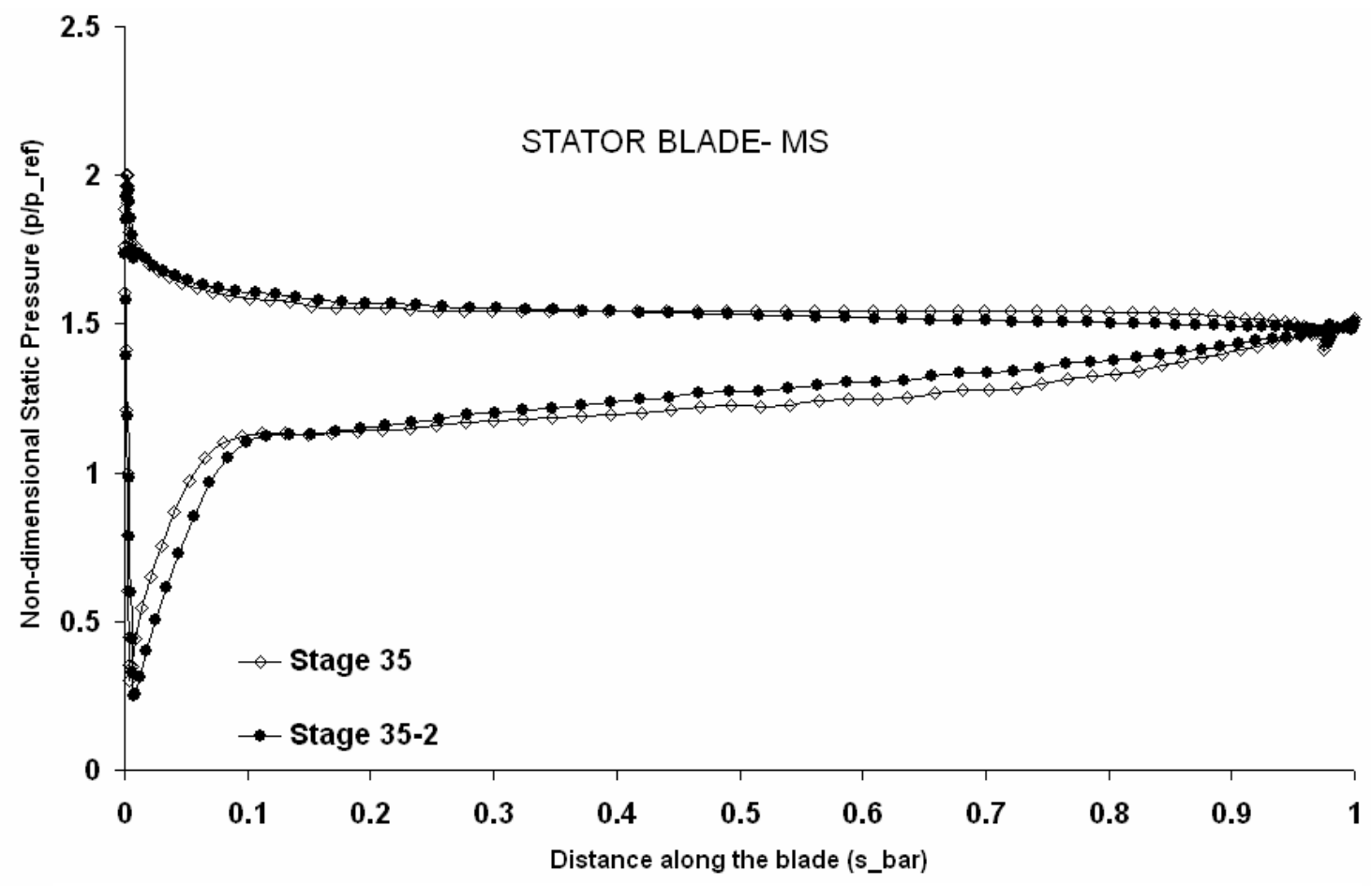

Figure 6.29: Stator blade loading curve comparison at mid span between the Stage 35 configuration and Stage 35-2 configuration. 


\section{CHAPTER VII}

\section{CONCLUSIONS AND RECOMMENDATIONS}

A first-principles based method to design axial compressor blade configurations has been developed. A systematic study of the effects of blade design parameters on compressor performance has been done. As a part of the work several aspects of blade design- aerodynamics, aeroacoustics and elastic behavior have been studied. A combination of performance map data and flow visualization studies have been used to report the findings. To the author's knowledge, this is the first multiobjective methodology that incorporates computational fluid dynamics and high fidelity design tools to facilitate design of axial compressor configurations.

In this chapter, conclusions of this research are presented in Section 7.1. The recommendations for further work in the future are given in Section 7.2.

\subsection{Conclusions}

Based on these studies, the following conclusions can be drawn:

1- The parametric design of an axial compressor configuration requires a number of advanced tools. The fidelity level of these tools dictates the accuracy and 
effectiveness of the design process. The operation of modern compressor processes is very complex and affected by nonlinear effects such as shocks, tip vortices, and blockages. High fidelity tools are therefore necessary. Such high fidelity tools have been extensively explored in this study.

2- In designing an axial compressor stage a large number of design variables are involved. These include design variables associated with the rotor and the stator blades, resulting in a large set of design of experiments. A systematic way to screen through the plethora of design variables based on their influence on the response(s) has been reported in this study. It was found that the variables associated with the rotor have a bigger influence on the response(s) compared to the stator blade variables.

3- Axial compressor blades are very sensitive to alterations in blade topology. It was found that by optimizing the blade leading edge and trailing edge mean camber line angles, phenomenon's such as tip blockages, blade-to-blade shock structures and other loss mechanisms can be weakened or alleviated. It was found that these can have a beneficial effect on total pressure ratio and stage adiabatic efficiency, therefore improving the performance of the axial compression system.

4- It was found that an aerodynamically optimized blade can also have aeroacoustic benefits. By optimizing the blade trailing edge, the fluctuating aeroacoustic signature from rotor to stator is minimized, therefore resulting in lower rotor wake-stator interactions. 
5- When designing an axial compressor blade, the blade deformations need to be addressed. More often than not these deformations are small, but they can have a significant effect on blade performance. It has been demonstrated that these deformations can be obtained from an open loop structural analysis.

\subsection{Recommendations}

Based on the calculation, the following recommendations are made for future study.

1- Although the blade parametric design tool CCGEOM was sufficient for this study, a need for a higher fidelity parametric design tool still exists. It is recommended that parametric design methods such the generic B-spline method.

2- It is recommended that other optimization methods such as Adjoint method and Evolutionary Algorithms be explored. Although some of these methods may be complicated to set up, these approaches will compliment the present approach.

3- $\quad$ In this study the blade parametric design was based on blade leading edge and trailing edge mean camber line angles at various chord and spanwise locations. In reality many more variables are involved- such as blade lean distribution, blade sweep distribution, chord distribution. Even though these were not investigated in this study; it is recommended that they be addressed 
for future work. Although if more design variables were to be explored then the number of experiments was to increase exponentially. Therefore a distributed processing capability is needed. It is recommended that the more design variables be explored to take advantage of the recent progress in distributed computation techniques.

4- Although only a single rotor-stator stage has been designed, the presently proposed methodology is applicable to multistage design. It is customary to first use a 1-D system analysis tool for the overall multistage design in order to get a starting configuration, the accuracy of which can then be verified by applying the present methodology to multiple stages. Such an analysis is recommended for further work.

5- Effects of changing end-wall contouring and rotor tip clearance gap on the performance system must be explored. This involves modeling the casing and incorporating the design variables associated with the casing in the design loop.

6- The distance between the axial distance between the rotor and stator can have a significant effect on the rotor wake and stator aeroacoustic interaction. It is recommended that this axial distance be explored as a design variable with a view to find an optimum distance from an aeroacoustic and aerodynamic perspective.

7- The present work has focused on coupling blade aerodynamics and blade aeroelasticity to account for static aeroelastic effects. In order to ensure a 
configuration that is free of transonic and/or stall flutter, it is necessary to tightly couple the aerodynamics of the blade to the aeroelastic behavior. The approach presented is well suited for a closed loop coupling with comprehensive aeroelastic solvers such as ANSYS and NASTRAN using a simultaneous integration of the solid and fluid dynamic equations. Further aeroelastic simulations are recommended.

It is hoped that this work gives a good understanding of challenges faced during design of an axial compressor configuration and that it will serve as a useful step for future investigations in the exciting area of compression system design. 


\section{REFERENCES}

1. Horlock, J. H., Axial Flow Compressors, Butterworths Scientific Publications, London, 1958.

2. Gostelow, J. P., Cascade Aerodynamics, Pergamon Press, New York, NY, 1984.

3. Hawthorne, W.R., Aerodynamics of Turbines and Compressors, Princeton University Press, Princeton, NJ, 1964.

4. Aungier, R. H., Axial-Flow Compressors, ASME Press, New York, 2003.

5. McKenzie, A. B., Axial Flow Fans and Compressors- Aerodynamic Design and Performance, Ashgate, England, 1997.

6. Oates, G. C., Aerodynamics Aircraft Engine Components, AIAA, New York, 1985.

7. Mattingly, J. D., Heiser W. H., Daley, D. H., Aircraft Engine Design, AIAA, New York, 1987.

8. Bashkarone, E. A., Principles of Turbomachinery in Air-Breathing Engines, Cambridge University Press, 2006.

9. Cumpsty, N. A., Compressor Aerodynamics, Longman Scientific and Technical, Essex, 1989.

10. Lakshminarayana, B., Fluid Dynamics and Heat Transfer of Turbomachinery, John Wiley and Sons. Inc, 1996.

11. Savage, M., Beatty, L. A., "Investigation of a Three-stage Transonic Research AxialFlow Compressor Aerodynamic Design and Overall Performance," NASA Technical Paper- NACA-RM-L55G27, 1955.

12. Schwenk, F. C., Lewis, G. W., Hartmann, M. J., "A Preliminary Analysis of the Magnitude of Shock Losses in Transonic Compressors," NASA Technical PaperNACA-RM-E57A30, 1957. 
13. Weinberg, B. C., Yang, R.-J., McDonald, H., and Shamroth, S. J., "Calculations of Two- and Three-Dimensional Transonic Cascade Flow Fields Using the NavierStokes Equations,: J. Eng. Gas Turbines and Power, Vol. 108, Jan., pp. 93-102, 1986.

14. Davis, R. L., Hobbs, D. E., and Weingold, H. D., "Prediction of Compressor Cascade Performance Using a Navier-Stokes Technique,” ASME Paper 88-GT-96, International Gas Turbine Conference, Amsterdam, The Netherlands, June 1988.

15. Gundy-Burlet, K. L., Rai, M. M., and Dring, R. P., "Two-Dimensional Computations of Multi-stage Compressor Flows Using a Zonal Approach," AIAA Paper 89-2452, Monterey, Calif., July 1989.

16. Chevrin, P. A., and Vuillez, C., "Viscous Flow Computations in Turbomachine Cascades," ASME Paper 90-GT-76, June 1990.

17. Cambier, L., Escande, B., and Veuillot, J. P., "Computation of Internal Flows at High Reynolds Number by Numerical Solution of the Navier-Stokes Equations," Rech. Aerosp., No. 1986-6, pp. 27-44, 1986.

18. Boyle, R. J., "Navier-Stokes Analysis of Turbine Blade Heat Transfer," ASME Paper 90-GT-42, June 1990.

19. Suder, K, L., and Celesttina, M. L., "Experimental and Computational Investigation of the Tip Clearance Flow in a Transonic Axial Compressor," ASME Paper 94-GT365, June 1994.

20. Chima, R. V., "Calculation of Tip Clearance Effects in a Transonic Compressor Rotor," NASA TM 107216, May 1996.

21. AGARD, "Test Cases for Computation of Internal Flows in Aero Engine Components," AGARD Propulsion and Energetics Panel, Working Group 18, AGARD-AR-275, 1989. 
22. Srivastava, R., and Sankar, Lakshmi N., "Efficient Hybrid Scheme for the Analysis of Counter-Rotating Propellers," Journal of Propulsion and Power, Vol. 9, No. 3, pp. 382-388, May-Jun 1993.

23. Dawes, W. N., "Numerical Study of the 3D Flowfield in a Transonic Compressor Rotor with a Modeling of the Tip Clearance Flow," AGARD Conference Proceedings, No. 401, Neuilly Sur Seine, March 1987.

24. Adamczyk, J. J., Mulac, R. A., and Celestina, M. L., "A Model for Closing the Inviscid Form of the Average Passage Equations," ASME Paper 86-GT-227, June 1986.

25. Hathaway, M. D., and Wood, J. R., “Application of a Multi-Block CFD Code to Investigate the Impact of Geometry Modeling on Centrifugal Compressor Flow Field Predictions," Transactions of the ASME, Vol. 19, Oct 1997, pp. 820-830.

26. Niazi, S., Stein, A., and Sankar, L. N., "Development and Application of a CFD solver to the Simulation of Centrifugal Compressors," AIAA paper 98-0934, 1998.

27. Chima. R. V., "Explicit Multigrid Algorithm for Quasi-Three-Dimensional Viscous Flows in Turbomachinery," AIAA Journal of Propulsion and Power, Vol. 3, No. 5, Sept-Oct. 1987, pp. 397-405.

28. Chima, R. V., and Yokota, J. W., "Numerical Analysis of Three-Dimensional Viscous Internal Flow,” AIAA Journal, Vol. 28, No. 5, 1990, pp. 798-806.

29. Niazi, S., "Numerical Simulation of Rotating Stall and Surge Alleviation in Axial Compressors," Ph.D. Dissertation, Georgia Institute of Technology, Aerospace Engineering, July 2000.

30. Denton, J. D. “The Calculations of Three Dimensional Viscous Flow Through Multistage Turbomachines," ASME Paper 90-GT-19, June 1990.

31. Hall, E. J., “Aerodynamic Modeling of Multistage Compressor Flow Fields - Part 1: Analysis of Rotor/Stator/Rotor Aerodynamic Interaction," ASME paper 97- GT344, 1997. 
32. Chima, R. V., "Calculations of Multistage Turbomachinery Using Steady Characteristic Boundary Conditions," AIAA Paper 98-0968, Jan. 1998. Also NASA TM-1998-206613.

33. Niazi, S., Stein, A., and Sankar, L. N., "Numerical Study of Surge and Stall Alleviation in a High-Speed Transonic Fan Rotor," AIAA Paper 2000-0225, Jan. 2000 .

34. Zaki, M., Iyengar, V., Sankar, L. N., “Assessment of Rotor-Stator Interface Boundary Condition Techniques for Modeling Axial Flow Turbines,” AIAA Paper 2006-4619, July 2006.

35. Horlock, J. H., Denton, J. D., “A Review of Some Early Design Practice Using Computational Fluid Dynamics and a Current Perspective," Journal of Turbomachinery, Vol. 127, January 2005.

36. Howell, A. R., "The Present Basis of Axial Flow Compressor Design: Part 1Cascade Theory and Performance," Aeronautical Research Council R. and M. No. $2095,1942$.

37. Carter, A. D. S., and Hughes, H. P., "A Theoretical Investigation of the Effect of Profile Shape on the Performance of Aerofoil's in Cascade," Aeronautical Research Council R. and M. No. 2384, 1950.

38. Stanitz, J. D., "Design of Two-Dimensional Channels with Prescribed Velocity Distributions along the Channel Walls," NASA Tech. Notes 2593-2595, 1952.

39. Gostelow, J. P., "Potential Flow through Cascades-Extension to an Exact Theory," Aeronautical Research Council, CP 808, 1962.

40. Hobson, D. E., "Shock Free Transonic Flow in Turbomachinery Cascade," Cambridge University Report, CUED/A Turbo 65, 1979.

41. Hawthorne, W. R., "Secondary Circulation in Fluid Flow," Proceedings Royal Society London, Ser. A, 206, p. 374, 1951. 
42. Smith, L. H., "Secondary Flow in Axial Flow Turbomachinery," Transactions ASME, 77, p.1065, 1953.

43. Lakshminarayana, B., Horlock, J. H., "Leakage and Secondary Flow in Compressor Cascades," Aeronautical Research Council, R. and M. 3483, 1967.

44. Lakshminarayana, B., "Predicting the Tip Clearance Flow in Axial Flow Turbomachines,” ASME, J. Basic Engineering, 92, pp467-482, 1970.

45. Katsanis, T., “A Computer Program for Calculating Velocities and Streamlines for Two-Dimensional, Incompressible Flow in Axial Blade Rows,” NACA Technical Note, NASA TN D- 3762, January 1967.

46. Murman, E. M., Cole, J. D., "Calculation of Plane Steady Transonic Flows," AIAA Journal 9: 114-21, 1971.

47. Jameson, A., "Numerical Calculations of the Three-Dimensional Flow over a Yawed Wing," Proceedings of the AIAA Computational Fluid Dynamics Conference, Palm Springs, July 1973, pp. 18-26.

48. Dulikravich D. S., "CAS2D - FORTRAN Program for Nonrotating Blade-to-Blade, Steady, Potential Transonic Cascade Flows,” NASA TN 1705, July 1980.

49. Meauze, G., "An Inverse Time Marching Method for the Definition of Cascade Geometry," Transaction of the ASME: J. of Engineering for Power, Vol. 104, pp650656, 1982.

50. Dulikravich D. S., "Shape Inverse Design and Optimization for Three-Dimensional Aerodynamics," AIAA paper 95-0695, AIAA Aerospace Sciences Meeting, Reno, NV, January 9-12, 1995.

51. Bolger, J. J., and Horlock, J. H., "Predictions of the Flow in Repeating Stages of Axial Compressors Using Navier-Stokes Solvers,” ASME Paper 95-GT-199, 1995. 
52. Hah, C., and Wennerstrom, A. J., "Three-Dimensional Flowfields Inside a Transonic Compressor with Swept Blades," Journal of Turbomachinery, Vol. 113, pp. 241-251, 1991.

53. Hah, C., Putterbaugh, S. L., and Wadia, A. R., "Control of Shock Structure and Secondary Flow Field Inside Transonic Compressor Rotors Through Aerodynamic Sweep," ASME Paper 98-GT-561, 1998.

54. Wadia, A. R., Szucs, P. N. and Crall, D. W., "Inner Workings of Aerodynamic Sweep,” ASME Journal of Turbomachinery, Vol. 120- No. 4, pp. 671-682, 1998.

55. Denton, J.D., amd Xu, L., "The Effects of Lean and Sweep on Transonic Fan Performance,” ASME Paper GT-2002-30327, 2002.

56. Dang, T. Q., “A Fully Three-Dimensional Inverse Method for Turbomachinery Blading in Transonic Flows," ASME Journal of Turbomachinery, vol. 115, no. 2, April 1993, pp. 354-361.

57. Dang, T. Q., Nerurkar, A. C. and Reddy, D. R., "Design modification of Rotor 67 by 3D Inverse Method- inviscid-flow limit,” ASME Paper 97-GT-484, 1997.

58. Zangeneh, M., Hawthorne, W. R. "A fully compressible three dimensional inverse design method applicable to radial and mixed-flow Turbomachines". 35th ASME/IGTI International Gas Turbine conference, Brussels. ASME Paper 90-GT198, pp.1-9, 1990.

59. Hu, P., and Zangeneh, M., "On Design of Transonic Fan Rotors by 3D Inverse Design Method," Proceedings of GT2006, ASME Turbo Expo 2006, GT2006-91173.

60. Keshin, A., Dutta, A. K., and Bestle, D., "Modern Compressor Aerodynamic Blading Process Using Multi-Objective Optimization," Proceedings of GT2006, ASME Turbo Expo 2006, GT2006-90206.

61. Gallimore, S. J., Bolger, J. J., Cumpsty, N. A., Taylor, M. J., Wright, P. I., Place, J. M. M., "The Use of Sweep and Dihedral in Multistage Axial Flow Compressor 
Blading-Part I: University Research and Methods Development" ASME Journal of Turbomachinery, Vol. 124, Issue 4, 2002.

62. Benini, E., and Biollo, R., "On the Aerodynamics of Swept and Leaned Transonic Compressor Rotors," Proceedings of GT2006, ASME Turbo Expo 2006, GT200690547.

63. Jang, C. M., Samad, A., and Kim, K. Y., "Optimal Design of Swept, Leaned and Skewed Blades in a Transonic Axial Compressor," Proceedings of GT2006, ASME Turbo Expo 2006, GT2006-90384.

64. Dunham, J., "A Parametric Method of Turbine Blade Profile Design," American Society of Mechanical Engineers, Paper 74-GT-119, 1974.

65. Korakianitis, T., "Design of Airfoils and Cascades of Airfoils," AIAA Journal, Vol. 27, No. 4, pp. 455-461, 1989.

66. Korakianitis, T., "Prescribed-Curvature-Distribution Airfoils for the Preliminary Geometric Design of Axial Turbomachinery Cascades," Journal of Turbomachinery, Vol. 115, pp. 325-333, April 1993.

67. Corral, R., and Pastor, G., "A Parametric Design Tool for Cascades of Airfoils," International Gas Turbine and Aeroengine Congress and Exhibition, American Society of Mechanical Engineers, Paper 99-GT-73, Indianapolis, Indiana, June 1999.

68. Corral, R., and Pastor, G., "Parametric Design of Turbomachinery Airfoils Using Highly Differentiable Splines," Journal of Propulsion and Power Vol. 20, No. 2, 2004.

69. Marvis, D. N., Mascotai, N. I., Roth, B., “A Probabilistic Design Methodology for Commercial Aircraft Engine Cycle Selection," AIAA-98-5510, $3^{\text {rd }}$ World Aviation Congress and Exposition, Anaheim, CA, 1998.

70. Roth, B., De Luis, J., "Lost Thrust Methodology for Gas Turbine Engine Performance Analysis," GT2005-68200, Proceedings of ASME Turbo Expo 2005, Reno, NV, USA, June 2005. 
71. Marvis, D. N., Roth, B., and Mascotai, N. I., "A Method for Probabilistic Sensitivity Analysis of Commercial Aircraft Engines," ISABE-99-BR, 14th ISABE, Florence, Italy, September 1999.

72. DeLaurentis, D., Mavris, D. N., and Schrage D. P., "System Synthesis in Preliminary Aircraft Design Using Statistical Methods," ICAS-96 20th International Council of the Aeronautical Sciences (ICAS) Congress, Sorrento, Italy, September 8-13, 1996.

73. Roth, B., Mavris, D. N., "A Work Availability Perspective of Turbofan Engine Performance," AIAA-2001-0391, 39th AIAA, Aerospace Sciences Meeting and Exhibit, Reno, NV, 2001.

74. Vanderplaats, G. N., "CONMIN A FORTRAN program for constrained function minimization User's manual,” NASA -TM-X-62282, 1973.

75. Hicks, R. M., and Henne, P. A., "Wing Design by Numerical Optimization," Journal of Aircraft, Vol. 15, pp. 407-412, 1978.

76. Reuther, J. J., and Jameson, A., "Supersonic Wing and Wing-body Shape Optimization Using an Adjoint Formulation," Technical Report, The Forum on CFD for Design and Optimization, (IMECE95), San Francisco, California, November 1995.

77. Lee, Y.-T., Luo, L., and Bein, T. W., "Direct Method for Optimization of a Centrifugal Compressor Vaneless Diffuser," Journal of Turbomachinery, Vol. 123, pp. 73-80, January 2001.

78. Koller, U., Monig, R., Kusters, B., and Schreiber, H.-A., "Development of Advanced Compressor Airfoils for Heavy-Duty Gas Turbines- Part I: Design and Optimization," Journal of Turbomachinery, Vol. 122, pp. 397-405, July 2000.

79. Yi, W., Huang, H., and Han, W., "Design Optimization of Transonic Compressor Rotor Using CFD and Genetic Algorithm," Proceedings of GT2006, ASME Turbo Expo 2006, GT2006-90155. 
80. Oyama, A., Liuo, M., and Obayashi, S., "Transonic Axial-Flow Blade Optimization: Evolutionary Algorithms/Three-Dimensional Navier-Stokes Solver," Journal of Propulsion and Power, vol. 20, No.4 July-August 2004.

81. Uelschen, M. and Lawerenz, M., "Design of Axial Compressor Airfoils with Artificial Neural Networks and Genetic Algorithms,” AIAA Paper 2000-2546, 2001.

82. Ahn, C. S., and Kim, K. Y., "Aerodynamic Design Optimization of a Compressor Rotor with Navier-Stokes Analysis," Proceeding of The Institution of Mechanical Engineers, Part A- Journal of Power and Energy, 217, No. 2, pp. 179-184.

83. Jang, C. M., and Kim, K. Y., "Optimization of a Stator Blade using Response Surface Method in a Single-Stage Transonic Axial Compressor," Proceeding of The Institution of Mechanical Engineers, Part A- Journal of Power and Energy 2005, vol. 219, no 8, pp. 595-603.

84. Envia, Edmane, "Fan Noise Reduction: An Overview," NASA Technical Paper, TM2001-210699.

85. Rao, G. V. R., "Use of Leaning Vanes for Fan Noise Reduction," AIAA Paper 72$126,1972$.

86. Heidelberg, L. J., "An Overview of the Fan Active Noise Control Effort at NASA Glenn," Presented at Joint Meeting of ASA, EAA and DEGA, Berlin, Germany, March 1999.

87. Tyler, J. M., and Sorfin, T. G., "Axial Flow Compressor Noise Studies,” 345D, Soc. Automotive Engrs., 1961.

88. Schulten, J. B. H. M., "Sound Generated by Rotor Wakes Interacting with a Leaned Vane Stator,” AIAA Journal, No. 10, 1352-1358, 1982.

89. Envia, E. and Kerschen, E. J., "Influence of Vane Sweep on Rotor-Stator Interaction Noise," NASA/CR 187052, 1990.

90. Envia, E. and Nallasamy, M., "Design Selection and Analysis of a Swept and Leaned Stator Concept," Journal of Sound and Vibration 228: 793-836, 1999. 
91. Envia, E., Huff, D., and Morrison, C. R., "Analytical Assessment of Stator Sweep and Lean in Reducing Rotor-Stator Tone Noise,” AIAA Paper 96-1791, May 1996.

92. Elhadidi, B., Atassi, H. M., "Passive Noise Control by Blade Lean and Sweep," $10^{\text {th }}$ AIAA/CEAS Aeroacoustics Conference, AIAA 2004-2999.

93. Ferrecchia, A., Dawes, W. N., Dhanasekaran, P. C., "Compressor Rotor Wakes and Tone Noise Study," AIAA 2003-3328.

94. Chima, R. V., "Swift - Multiblock Analysis Code for Turbomachinery, User's Manual and Documentation," Version 300, Sept. 2003.

95. Tannehill, J. C., Anderson, D. A., Pletcher, R. H., “Computational Fluid Mechanics and Heat Transfer," Taylor and Francis, $2^{\text {nd }}$ Edition, 1997.

96. Giles, M. B., "Nonreflecting Boundary Conditions for Euler Equation Calculations," AIAA Journal, Vol. 28, No. 12. pp 2050-2058, Dec. 1990.

97. Jameson, A., Schmidt, W., and Turkel, E., "Numerical Solutions of the Euler Equations by Finite Volume Methods Using Runge-Kutta Time-Stepping Schemes," AIAA Paper 81-1259, June 1981.

98. Jameson, A., "Solution of the Euler equations by a Multigrid Method," Appl. Math. Comput. 13, 327, 1983.

99. Martinelli, L., "Calculations of Viscous Flows with a Multigrid Method," Ph.D. thesis, MAE Department, Princeton Univ., 1987.

100. Wilcox, D., C., "Turbulence Modeling for CFD," DCW Industries, Inc. La Canada, CA, 1994.

101. Chima, R. V., “A k- $\omega$ Turbulence Model for Quasi-Three-Dimensional Turbomachinery Flows,” AIAA Paper 96-0248, 1995. Also NASA TM-107051.

102. Menter, F. R., "Improved Two-Equation k- $\omega$ Turbulence Model for Aerodynamic Flows,” NASA TM-103975, Oct. 1992. 
103. Chima, R. V., “TCGRID 3-D Grid Generator for Turbomachinery, User's Manual and Documentation," Version 300, July, 2003.

104. Steger, J. L., and Sorenson, R. L., "Automatic Mesh-Point Clustering Near a Boundary in Grid Generation with Elliptic Partial Differential Equations," J. Comp. Physics, Vol. 33, No. 3, Dec. 1979, pp. 405-410.

105. Wood, J. R., “CCGEOM User Manual,” Feb. 1997.

106. Lawson, J. S., and Madrigal, J.L., "Robust Design Through Optimization Techniques," Quality Engineering, 6, 593-608.

107. Harrington, Jr. E. C., "The Desirability Function," Industrial Quality Control, Volume 21, No. 10, pp. 494-498.

108. Smith, M. J. T., "A Look into the Aero Engine Noise Problem," Journal of Physics Education, Volume 6, Number 4, pp. 193-201(9), 1971.

109. Cotter, S., "A Screening Design for Factorial Experiments with Interactions," Biometrika, Vol. 66, pp. 317-320, August 1979.

110. Nixon, J. N., “A Systematic Process for Adaptive Concept Exploration,” PhD Thesis, Georgia Institute of Technology, Dec. 2006.

111. Ferguson, G. A., Takane, Yoshio. "Statistical Analysis in Psychology and Education", Sixth Edition. Montréal, Quebec, McGraw-Hill Ryerson Limited, 2005 .

112. Lindman, H. R. "Analysis of variance in complex experimental designs". San Francisco, W. H. Freeman \& Co, 1974.

113. Kirby, M. R., “TIES for Dummies”, Reference Manual, $3^{\text {rd }}$ Edition, 2002.

114. Strazisar, A.J., Wood, J. R., Hathaway, M. D., Suder, K. L., "Laser Anemometer Measurements in a Transonic Axial Flow Fan Rotor," NASA TP-2879, Nov. 1989. 
115. Reid, L., Moore, R. D., "Design and Overall Performance of Four Highly-Loaded, High-Speed Inlet Stages for an Advanced, High-Pressure-Ratio Core Compressor," NASA TP-1337, 1978.

116. Goldman, L. J., and McLallin, K. L., "Cold-Air Annular-Cascade Investigation of Aerodynamic Performance of Core-Engine-Cooled Turbine Vanes I: Solid-Vane Performance and Facility Description," NASA TMX-3224, 1975.

117. Yeuan, J. J and Hamed, A., "Three-Dimensional Viscous Flow Simulations in a Turbine Stator Using A Nonperiodic H-Type Grid,” AIAA Paper 98-3562.

118. Hudson, S. T., Gaddis, S. W., Johnson, P. D., and Boynton, J. L., "Cold Flow Testing of the Space Shuttle Main Engine High Pressure Fuel Turbine," AIAA Paper 91-2503, June 1991.

119. Dunn, M. G., Kim, J., Civinskas, K. C., and Boyle R. J., "Time-Averaged Heat Transfer and Pressure Measurements and Comparison with Prediction for a TwoStage Turbine," J. Turbomachinery, Vol. 116, Jan. 1994, pp. 14-22. 


\section{VITA}

\section{VISHWAS IYENGAR}

Vishwas Iyengar was born in Bangalore, India on $21^{\text {st }}$ March 1980. He attended Delhi Public School in New Delhi, India for 16 years, after which he attended Watford Grammar School in Watford, England for 2 years. He received a joint B.Eng / M.Eng. in Aeronautical Engineering from University of Durham, England in 2002 before coming to Georgia Tech to pursue a doctorate in Aerospace Engineering. During his time as a $\mathrm{Ph} . \mathrm{D}$. student he served as graduate research assistant for Dr Lakshmi Sankar and also as a grader for undergraduate courses.

He has submitted and presented several papers in conferences and symposiums He is a student member of American Society of Mechanical Engineers.

When he is not working on his research, Vishwas enjoys modeling and salsa dancing. He has given several salsa performances around the city of Atlanta. He has also been featured in billboards and advertisements in Atlanta as a model. Vishwas is a foreign film enthusiast, and in his spare time he also enjoys playing cricket and soccer, cooking, wine-tasting, short-story writing and traveling. 